\title{
Unpacking contemporary English blends: Morphological structure, meaning, processing
}

\author{
by
}

Natalia Beliaeva

\author{
A thesis \\ submitted to the Victoria University of Wellington \\ in fulfilment of the requirements for the degree of \\ Doctor of Philosophy
}

Victoria University of Wellington

(2014) 



\section{Abstract}

It is not coincidental that blend words (e. g. nutriceutical $\leftarrow$ nutricious + pharmaceutical, blizzaster $\leftarrow$ blizzard + disaster) are more and more often used in media sources. In a blend, two (or sometimes more) words become one compact and attention-catching form, which is at the same time relatively transparent, so that the reader or listener can still recognise several constituents in it. These features make blends one of the most intriguing types of word formation. At the same time, blends are extremely challenging to study. A classical morpheme-based morphological description is not suitable for blends because their formation does not involve morphemes as such. This implies two possible approaches: either to deny blends a place in regular morphology (as suggested in Dressler (2000), for example), or to find grounds for including them into general morphological descriptions and theories (as was done, using different frameworks, in López Rúa (2004b), Gries (2012), Arndt-Lappe and Plag (2013) and other studies). The growing number of blends observed in various media sources indicates that this phenomenon is an important characteristic of the living contemporary language, and therefore, blends cannot be ignored in a morphological description of the English language (and many other typologically different languages). Moreover, I believe that the general morphological theory has to embrace blends because of the vast amount of regularity observed in their formation, despite their incredible diversity.

The formation of blends involves both addition and subtraction, which relates them both to compounds and to clippings. This research aims to clarify the morphological status of blends in relation to the neighbouring word formation categories, in particular, to the so-called clipping compounds (e.g. digicam $\leftarrow$ digital + camera). To approach this problem, I compiled a collection of English neologisms formed by merging two (in some cases, more) words into one, and analysed their formal and semantic properties. The results of this analysis were used to distinguish between blends and clipping compounds, and also to justify the classification of blends according to different degrees of formal transparency (using the principles of Lehrer's (1996, 2007) classification). The strength of the association between blends (or clipping compounds) and their source words was then assessed in two experiments: an online survey involving evaluating definitions of blends and clipping compounds, and a psycholinguistic experiment involving a production and a lexical decision task. The 
experimental findings show that recognisability of the source words of blends and clipping compounds has significant influence both on the evaluation of their definitions and on their processing. The main implication of the experimental results is that blends, unlike clipping compounds, are closer to compounds than to clippings. In addition to this, significant differences are revealed between blends containing full source words and blends containing only parts of them. Therefore, the structural type of blend, as defined in this study, is a factor which has strong influence on the processing of blends and their source words. 


\section{Acknowledgements}

This thesis primarily concerns my contribution to the knowledge about blends. This contribution, however, seems tiny in comparison to the amount of knowledge and experience I acquired during my $\mathrm{PhD}$ journey. These were given to me by the people who surrounded me at various stages of this project to a much greater extent than taken from books and journals. To all these people, I express my heartfelt gratitude.

First of all, I am grateful to my primary supervisor, Professor Laurie Bauer, who did much more for this research project than fulfil his supervisory duties. His friendly support, inspiring feedback, and his genuine interest not only in the formation of blends, but also in my own formation as an independent researcher have been crucial for the progress of this thesis. I am also indebted to my secondary supervisor, Associate Professor Paul Warren, who provided extremely valuable feedback at all the stages of the experimental part of this project, and whose comments on my writing inspired me to address the trickiest aspects of statistical analysis instead of avoiding them. I am further grateful to Dr. Anna Siyanova, who agreed to join the supervisory team when this project was well under way. Her expertise in experimental studies was very valuable for shaping my own experimental methodology. I am also very grateful to her for positive attitude to all aspects of my work, which was vital for getting me through bumps on this road. I would like to add a special thank you to Professor Ingo Plag, who provided stimulating feedback and advice at a crucial stage of my $\mathrm{PhD}$, and who introduced me to the exciting world of statistical analysis in R.

I also wish to thank my examiners Prof. Jen Hay, Dr Paula López Rúa and Dr. Carolyn Wilshire for their genuine interest in my work and their insightful comments.

I would like to thank all the staff of School of Linguistics and Applied Language Studies for being a lively, inspiring and supportive academic community. I also thank the School for providing opportunities to gain teaching experience, and I must confess that I learned at least as much from the courses I worked on as the students did. I am grateful to all the teaching and the administrative staff of SLALS for making life at the School easier in all sorts of ways, from sorting out administrative issues smoothly and almost unnoticeably, to relaxing conversations over innumerable cups of coffee we had together. And, of course, I am sincerely grateful to my fellow PhD students Anna Piasecki, Sharon Marsden, Ewa Kusmierczyk, Kieran File, Keely Kidner, TJ Boutorwick, Deborah Chua and many others, who provided valuable feedback during the exciting sessions of the thesis 
group, and who helped make Wellington my second home. A special thanks to Liza Tarasova, for making my landing in New Zealand as soft as it could possibly be.

This research project would not have been possible without the financial support of the grant from the Royal Society of New Zealand through its Marsden Fund to Laurie Bauer, and also without the research grants of the Faculty of Humanities and Social Sciences.

Undertaking this research project would be much less exciting and much more solitary without the support of my family and friends both in New Zealand and in Russia. I would like to thank Dr Elena Myagkova, who first inspired my interest in linguistic research, and Dr Vera Pishchalnikova, my mentor and supervisor of the Candidate of Philological Sciences thesis. Words can hardly express my gratitude to my parents for their love and support, and to my husband Aleksandr and my daughter Polina for constantly reminding me there is life outside the $\mathrm{PhD}$.

You gave me much more than I could ever give back. Thank you. 


\section{Papers and presentations derived from this research}

\section{Journal papers:}

Beliaeva, N. (2014). A study of English blends: From structure to meaning and back again. Word Structure, 7(1), 29-54.

\section{Conference presentations:}

Beliaeva, N. (2012). The chemistry of blends: How people merge words together. Presented at the $2^{\text {nd }}$ Auckland Postgraduate Conference on Linguistics and Applied Linguistics, Auckland, June 30.

Beliaeva, N. (2012). The power of slanguage: Form and meaning of English blends. Presented at the Data-Rich Approaches to English Morphology, Wellington, July 4-6.

Beliaeva, N. (2012). The power of slanguage: Conceptual integration on the word formation level. Presented at the $4^{\text {th }}$ UK Cognitive Linguistics Conference, London, July $10-12$.

Beliaeva, N. (2013). From phonology to morphology, from morphology to semantics: A study of English blends. Presented at Morphology and its Interfaces, Lille, September $12-13$. 


\section{Contents}

Abstract

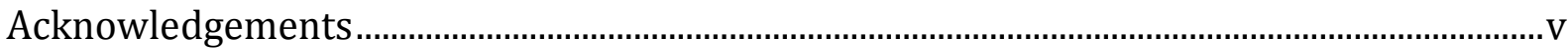

Papers and presentations derived from this research..............................................................vii

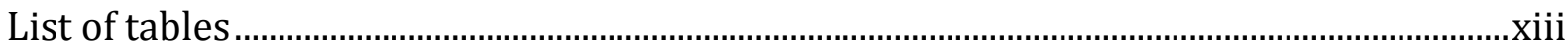

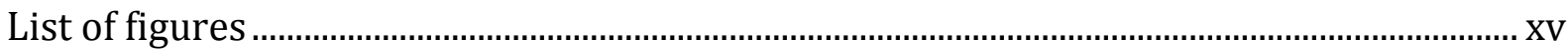

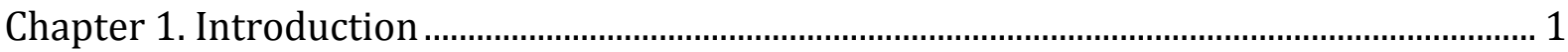

1.1. Background and motivation of the thesis ........................................................................ 1

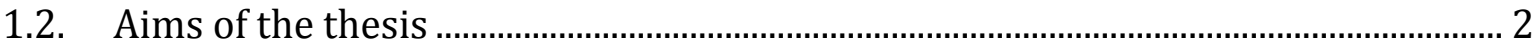

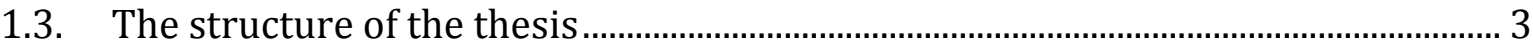

Chapter 2. The dramality of the blendaverse: Research on blends .......................................... 5

2.1. Early classifications and classical discrepancies .......................................................... 5

2.2. A closer look at the mechanism of blending...............................................................

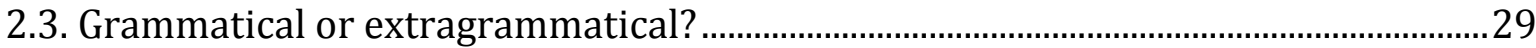

2.4. The position of blends among the neighbouring morphological categories .............31

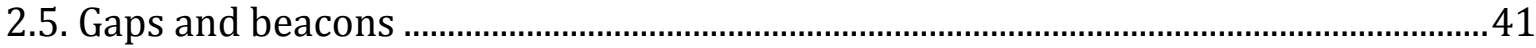

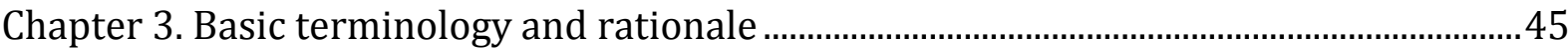

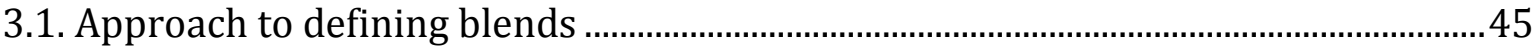

3.2. The terminological toolkit and the scope of the study …...................................................47

Chapter 4. Lexical data: From structure to meaning and back again .....................................51

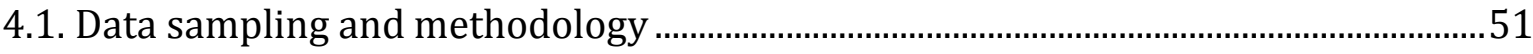

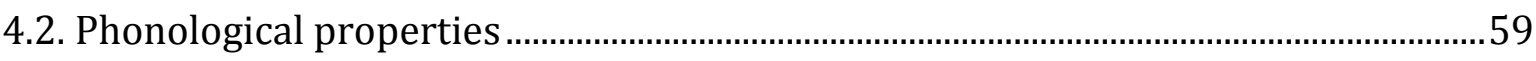

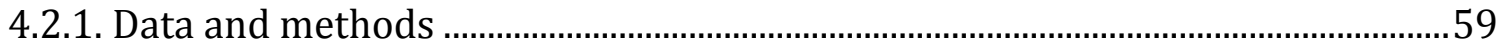

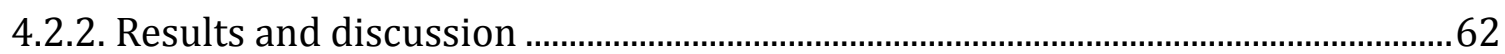

4.3 Structural properties: Interaction with phonology ......................................................68

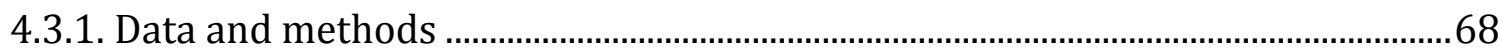

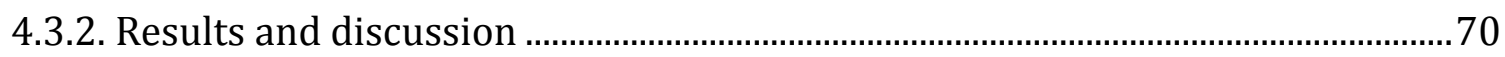

4.4. Semantic properties: Interaction with structure ........................................................ 75 


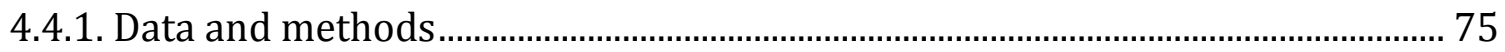

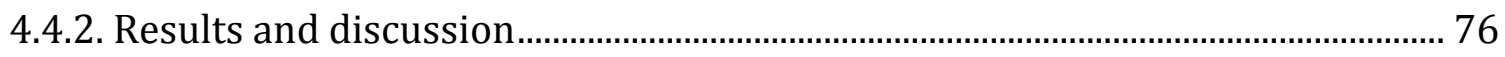

4.5. Interim conclusions: Phonological and semantic factors which influence the structure of blends 80

Chapter 5. Deconstructing blends: Insights from psycholinguistic and cognitive studies 85

5.1. Cognitive mechanisms of blending revealed in the form of blends............................ 85

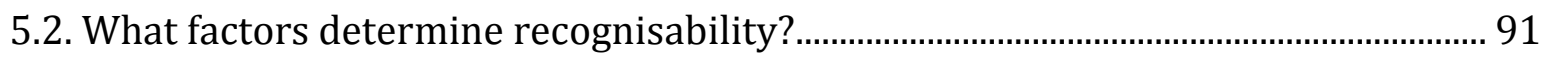

5.3. Methodological prerequisites for an experimental study of blends ......................... 102

Chapter 6 . What can be predicted from the way predictionary and other blends are

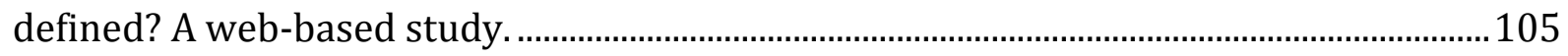

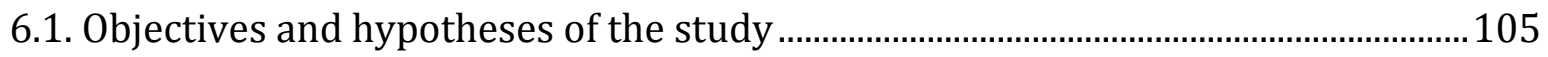

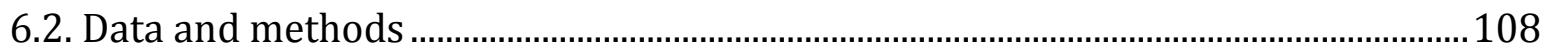

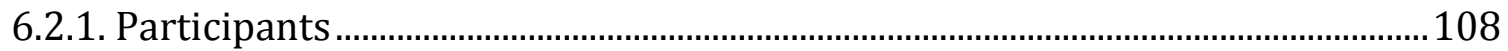

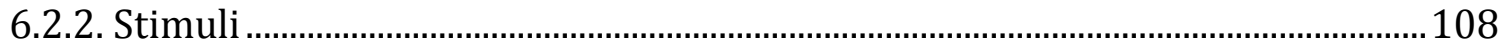

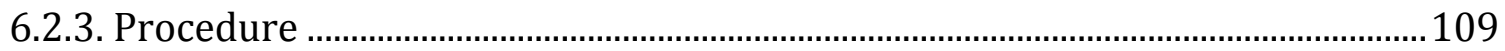

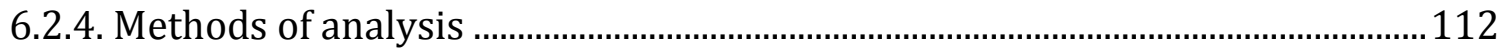

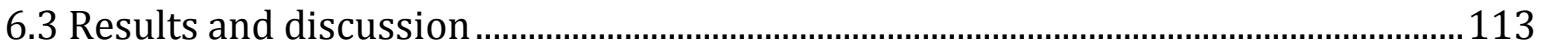

6.4. Interim conclusions: Perception and understanding of blends and clipping compounds.

Chapter 7. Can you find an academic in an acatramp? Priming effect of blends and clipping compounds on the processing of their source words.

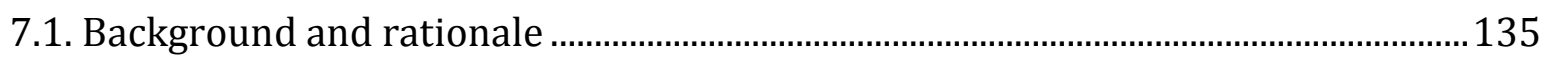

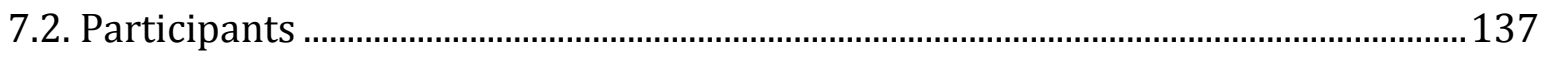

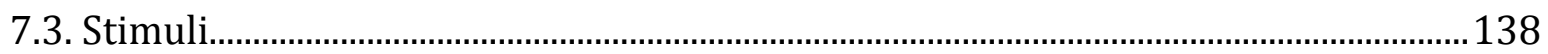

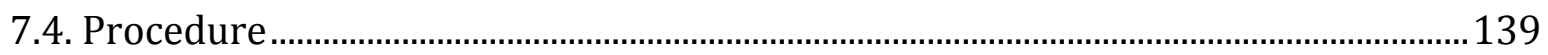

7.4.1. Task 1: An identification and production task ...................................................... 139

7.4.2. Task 2: A lexical decision task .................................................................................. 141

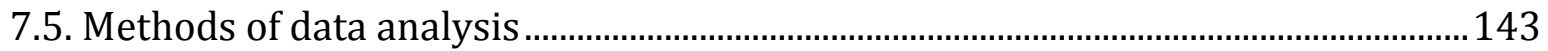


7.5.1. Task 1

7.5.2. Task 2

7.6. Results 146

7.6.1. Identification and production task results 146

7.6.2. Lexical decision task results 158

7.7. Interim conclusions: Recognisability and recognition 170

Chapter 8. Synthesis and conclusions 175

8.1. The design of this research revisited 175

8.2. The main findings of the thesis 176

8.3. The implications of the research 184

8.3.1. Finding room for blends in English morphology: Implications for taxonomic studies 184

8.3.2. Finding room for blends in your 'mind palace': Implications for the studies of

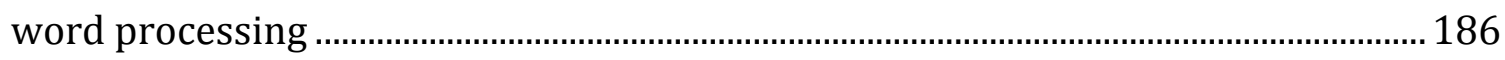

8.4. The limitations of the study and the recommendations for future research ........ 188

References 191

Appendix 1. Lexical data 201

Appendix 2. Ethics Approval 213

Appendix 3. Information sheet for participants of the web-based survey. 215

Appendix 4. Survey stimuli 217

Appendix 5. Item variables used in the survey . 233

Appendix 6. Regression models predicting the responses in the survey 235

Appendix 7. Information sheet for the participants of the experiment. 249

Appendix 8. Consent form for the participants of the experiment 251

Appendix 9. Experimental stimuli. 253

Appendix 10. Item variables used for the analysis of experimental data 255 Appendix 11. Regression models predicting the response to the experimental stimuli 257 


\section{List of tables}

Table 1. Derivatives and compounds formed by adding recently established morphs ....52

Table 2. Structural classification of lexemes in the collected corpus ......................................56

Table 3. Structural classification of lexemes in the collected corpus, revised .....................58

Table 4. Structural types of blends with non-central overlap .................................................59

Table 5. The syllable structure of the four main types of blends ............................................ 70

Table 6.The distribution of the data with regard to the semantic origin and the type of source word combinations attested in COCA.............................................................................

Table 7. Source words combinations extracted from COCA …….............................................77

Table 8. Survey stimuli

Table 9. Observed median responses to definitions of different types of target words 116 Table 10. Likelihood ratio tests comparing models predicting the response in the main survey with increasingly complex random effects structure

Table 11. The effects of item and participant factors on the response in the main survey

Table 12. Likelihood ratio test comparing the model with simple effects of blend type and definition type with the model including the interaction of the two factors.....

Table 13. The effects of blend type and definition type on the response in the main survey

Table 14. Likelihood ratio test comparing the model with simple effects of blend type and definition type with the model including the interaction of the two factors (combined data from the main and the additional survey)

Table 15. Simple effects of blend type and definition type in the main and the additional survey

Table 16. The effects of blend type and definition type, and the interaction of the two factors in the main and the additional survey

Table 17. Arrangement of word stimuli into groups according to priming conditions. 142

Table 18. Percentage of correct answers in Task 1

Table 19. The output of the regression model predicting SW1 naming. 148

Table 20. The output of the regression model predicting SW2 naming. 148

Table 21. The output of the regression model predicting SW2 naming in Group 1 149

Table 22. Likelihood ratio tests comparing models with splinter frequency and relative frequency predicting SW1 naming. 
Table 23. Likelihood ratio tests comparing models with splinter frequency and relative frequency predicting SW2 naming.

Table 24. Likelihood ratio tests comparing models with correlating predictors of SW1 naming.

Table 25. The output of the final model predicting SW1 identification 153

Table 26. Likelihood ratio tests comparing models with correlating predictors of SW2 naming. 155

Table 27. The output of the final model predicting SW2 identification. 156

Table 28. The output of the model predicting RT in Task 1 157

Table 29. Mean reaction time to target words in Task 2 for different priming conditions, millisecs (SD). 160

Table 30. The output of the model predicting RT to words in Task 2, with simple effects only 161

Table 31. The output of the model predicting RT to words in Task 2, for

PrimeShownTRUE condition. 162

Table 32. Likelihood ratio tests comparing models with increasingly complex structure 163

Table 33. The output of the final model predicting RT to words in Task 2 .........................163

Table 34. Mean reaction time to nonwords in Task 2, millisecs (SD)..................................165

Table 35. Likelihood ratio tests comparing models with increasingly complex structure 166

Table 36. The output of the final model predicting RT to nonwords in Task 2 ................166

Table 37. The proportion of errors in Task 2 for different priming conditions, \%..........169

Table 38. The output of the model predicting error rate for words in Task 2 .................. 169

Table 39. The output of the model predicting error rate for nonwords in Task 2 ...........170 


\section{List of figures}

Figure 1. Linguistic constraints on blend formation.

Figure 2. The proportion of the first (SW1) and the second (SW2) source words preserved in formations of different structural types. 60

Figure 3. Boundary phonemes in blends and clipping compounds.

Figure 4. Correlation between the length of the splinters and the length of the source words.

Figure 5. Correlation between the length of the left splinter of the first source word (W1) and the frequency rank of the boundary phonemes.

Figure 6. Correlation between the length of the right splinter of the second source word (W2) and the frequency rank of the boundary phonemes.

Figure 7. Correlation between the length of the left splinter of the second source word (W2) and the frequency rank of the boundary phonemes in AC formations. .66

Figure 8. The results of the decision tree analysis of the influence of the switch point placement and the phonological overlap on the structural type of a blend in the YN group.

Figure 9. The prosodic pattern of source words repeated in a blend as the predictor of the structural type of the blend

Figure 10. Frequency and type of source word combinations as the predictor of the semantic type of blends.

Figure 11. Frequency and type of source word combinations as the predictors of the structural type of blends.

Figure 12. The distribution of responses by the age group of the participants (a) and by their education level (b).

Figure 13. The distribution of responses by structural type of the target word (a) and by definition type (b)

Figure 14. A decision tree analysis of the response with item factors as independent variables.

Figure 15. A decision tree analysis of the response to the main survey, with item and participant factors as independent variables.

Figure 16. A decision tree analysis of the response to the main survey, with item factors as independent variables.

Figure 17. A quantile-to-quantile plot of the residuals of the regression model predicting the responses in the main survey. 
Figure 18. Regression coefficients for response by blend type and definition type, in the model with interaction of these experimental variables 128

Figure 19. Regression coefficients for response by blend type and definition type, in the model with interaction of these experimental variables 130

Figure 20. The experimental procedure: Task 1 ….....................................................................140

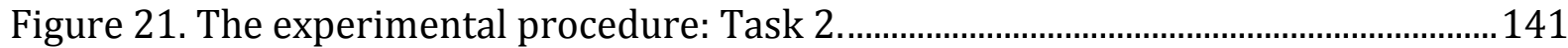

Figure 22. Correlation matrix of a selection of item variables. 150

Figure 23.The interaction of prime type and the relative frequency of SW1 splinter in the model predicting SW1 naming in Task 1 154

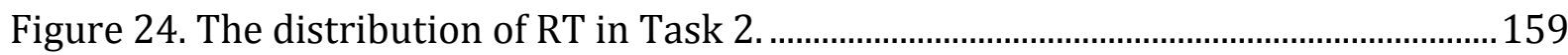

Figure 25. Correlation matrix of a selection of target variables. 162

Figure 26. The interaction of prime type and priming condition in the model predicting RT in Task 2.

Figure 27. The interaction of prime type and priming condition in the model predicting RT to word targets (left panel) and nonword targets (right panel) in Task 2. 168 


\section{Chapter 1. Introduction}

\subsection{Background and motivation of the thesis}

A blend word used in advertisement (e.g. nutriceutical) or in a title (e.g. Lehrer's (2007) Blendalicious) is both attention-catching and thought-provoking. Putting together two words to form a compound such as sugar bowl is one of the most straightforward ways to form a new lexeme. A more complex and less frequent way of making one word from two (or sometimes more, e.g. Christmahanukwanzadan) is merging them together so that part of the material is lost in the process. Blends are formed in such a way that a well-formed blend has the phonotactic structure of a simplex word, as observed, for example, in Tomaszewitz (2012). At the same time, the constituent words remain recoverable from the form of the blend (Gries, 2004a). These, among other, properties of blends make them one of the most intriguing types of word formation. It has to be noted that there is no agreement in the linguistic literature as to whether or not blending is a productive process of regular word formation. One of the arguments to the contrary is that the exceptional formal diversity of blends makes it appear that their formation is completely unpredictable.

The considerations above explain why blends are extremely challenging to study. A classical morpheme-based morphological description is not suitable for blends because their formation does not involve morphemes as such. This situation implies two possible approaches: either to deny blends a place in regular morphology, or to adjust morphological description in order to embrace this phenomenon. The literature has examples of both approaches. On the one hand, blends have been analysed as irregular, creative formations (assuming that creativity is opposed to morphological productivity, following Bauer's (2001, p. 64) terminology), and hence excluded from morphological analysis (Dressler, 2000; Mattiello, 2013). On the other hand, the surface structure of blends, their phonology and semantics have been analysed in order to find grounds for including them in general morphological descriptions. For example, the mechanisms of blending have been investigated within constraint-based theoretical frameworks such as Optimality Theory (Arndt-Lappe and Plag, 2013) and Schema Theory (Kemmer, 2003).

With regard to the relationship between blends and other word formation types, one way of classifying them is as an intermediate link between compounding and clipping (López Rúa, 2004b), or, in a wider sense, between productivity and creativity. Grey areas 
like these, although difficult to research and yielding controversial results, can provide insights into the different areas they adjoin. Hence, studying blends is not only intellectually provocative, but potentially theoretically and practically valuable.

\subsection{Aims of the thesis}

With the assumption that blends lie in a border region between several morphological categories, the primary aim of this research is to locate where exactly. In particular, the purpose of this study is to investigate whether blending as a word formation process is a type of compounding, a type of clipping, a combination of both processes, or neither of them. This, in turn, leads to a question of whether and to what extent blends are different from so-called clipping compounds which, like blends, have features in common with both compounding and clipping.

Analysing the literature on the topic reveals not only the different and often controversial views on blends mentioned above, it also makes clear that the definition of blends and the criteria for including lexemes in this category have changed considerably over time. Moreover, contemporary studies of blends are often based on lexical data from earlier publications, which can be problematic for two reasons. First, a lot of lexemes cited as blends in early studies may no longer be analysable as such because the semantic link between the blend and the blended words may no longer be salient. Second, the analysis of the blends, that is, the words they originate from, and the way they are blended, may be biased by the views of the researchers who collected the original data. The first aim of this research, therefore, is to describe blends as a morphological phenomenon as accurately and objectively as possible, and to make this description reflect the contemporary state of blends in the English language. Of course, it is not possible to avoid relying on earlier theoretical accounts and practical methods. At various stages of this study, I have made decisions driven by earlier findings, and adopted definitions and theoretical assumptions provided by earlier research. However, an important decision concerning my approach to data collection and analysis was to compile a collection of contemporary blends from original sources other than linguistic publications, and to analyse them as impartially as possible. Restricting the analysis to comparatively recent formations comes at the cost of losing a considerable amount of lexical data (many earlier studies analysed bigger collections of blends because they included well-established blends alongside new ones). However, the observations and 
generalisations made on the basis of neologisms can help to provide a more accurate account of the formation and functioning of blends in contemporary language.

It has often been pointed out in literature (e.g. Gries, 2006; Bauer, 2012) that providing a structural, phonological and/or semantic taxonomy of blends may not be a sufficient way of analysing them, particularly because they are so diverse. To understand the nature of this phenomenon, it is essential to consider the cognitive mechanisms that are responsible for blend formation and processing. In other words, to analyse blends adequately, it is important to know how language users process and analyse them.

In terms of processing blends, the question that is particularly important for this research concerns the strength of the link between a blend word and its constituent words. Presumably, if blending is a type of compounding, a blend should be processed like a unit made of two constituents. But, unlike compounds, blends lack some of the phonological and/or graphical material of their source words. If what remains is still enough to recover the full constituents of blends, then the formations for which such recovery is not possible (apart from lexicalised items which are, as I pointed out above, deliberately excluded from the scope of my research) are to be discarded from the category of blends, or at least prototypical blends. Lack of recognisability of constituents makes such formations more similar to acronyms than to compounds. Alternatively, if recoverability of the form and meaning of the source words is not a defining feature of blends, then they should be regarded as a subtype of complex clippings with a primary function of presenting several constituents in one compact form. With these considerations in mind, I designed and carried out two experiments aiming to access the strength of the association between blends and their source words. The significance of the analysis of these experiments is twofold. First, as I mentioned above, information about the processing of blends is valuable for their morphological description and classification. But, perhaps, more importantly, it is valuable as a contribution (one of many) to our knowledge about the representation and processing of words by language users.

\subsection{The structure of the thesis}

In this thesis, Chapter 2 provides a review of the morphological studies of blends over an extended period of time. The development of various approaches to defining and classifying blends is tracked, and gaps in the research are identified. 
In Chapter 3, the analysis of previous approaches to blends is used to work out a definition of them. The key terms to be used throughout the thesis are defined, and the scope of the research is outlined. Chapters 4-7 expound three different studies which illuminate the formation of blends from different perspectives. The studies were carried out successively so that foremost findings were used to specify more accurately the hypotheses and methods of the subsequent studies.

Chapter 4 is dedicated to the description and analysis of the phonological, structural and semantic features of blends. The chapter discusses the collection of over 500 English neologisms formed by merging two or, in some cases, more words into one. The collected neologisms are then classified in terms of formal and semantic regularities. The results of this analysis are used to distinguish between blends and clipping compounds, which supports earlier findings reported in Gries (2006). The results are also applied to justify the classification of blends according to different degrees of formal transparency (using the principles of Lehrer's $(1996,2007)$ classification).

Chapter 5 provides an introduction into cognitive and psycholinguistic approaches to studying blends, with a focus on the factors which may determine the recognisability of their original constituents. The analysis of a selection of experimental studies of word recognition is then used to outline the methodological and theoretical prerequisites of my own experimental study.

The experimental part of this research includes two stages. The first stage is a webbased survey in which readers evaluated definitions of blends and clipping compounds as more or less successfully explaining their meaning. Chapter 6 provides a description of the survey methods and procedure, and then discusses its results.

The final stage of the present research is a psycholinguistic experiment involving a production task and a lexical decision task. The objective of the experiment is to reveal, first, how successfully the readers of the blends and clipping compounds may retrieve their source words and, second, to what extent getting these words activated in a production task enhances the recognition of the same words in a lexical decision task. The design and procedure of the experiment, its results and implications are discussed in Chapter 7.

Chapter 8 recapitulates the main findings of this thesis, and provides a general discussion of its implications, limitations, and perspectives for future research. 


\section{Chapter 2. The dramality of the blendaverse: Research on blends}

The neologasm experienced by a linguist who comes across a good blend is often overshadowed by the puzzlement posed by the structure of blends in general. For instance, merging together the beginning of Twitter and the end of people gives the blend Tweople, and one could expect digital and camera to form a blend *digamera or *digera, following a similar pattern. However, instead of this, we attest digicam, which combines the beginnings of both words. The formal structure of these words has long been said to be unpredictable (e.g. Bauer 1983), and blending has been referred to, e.g. in Dressler (2000) or, later, in Mattiello (2013) as an extragrammatical process, rather than part of regular morphology. On the other hand, many recent studies of blends (López Rúa 2004a; Gries 2006, 2012; Lehrer 2007, to name just a few) have shown that the structure of blends is much more predictable than it might seem at first sight. For example, the formation of blends of the Tweople type is subject to such factors as the prosodic structure of their constituent words and their relative frequency. These factors, however, seem not to work in the same way with digicam, and for this reason, some classifications exclude these coinages, often called clipping compounds (Bauer 2012) or complex clippings (Gries 2006), from the category of blends. This chapter will explore the problem of the definition of blends and their delimitation from other morphological categories. As a starting point, the following section will focus on approaches to defining blends and will demonstrate that their definition is a subject of debate in the literature.

\subsection{Early classifications and classical discrepancies}

The word blend was not used as a linguistic term before the late $19^{\text {th }}$ century, and even then it did not mean what it means today. In the academic works of the late $19^{\text {th }}$ century the term was used mainly in the context of speech errors, e.g. Sweet in (1892: § 48) mentioned that blending of different constructions may cause certain grammatical and logical anomalies. The same use of the term can be seen in Jespersen (1918: 52): "Contaminations or blendings of two constructions between which the speaker is wavering occur in all languages". Here, grammatical confusions are meant, e.g. should better $+\mathrm{V}$ used instead of had better $+\mathrm{V}$ or should $+\mathrm{V}$. The use of term "blend" for phonologically or semantically based speech errors like needcessity (from need + necessity) can be tracked down to Meringer and Mayer (1895).

It is in the linguistic works of early $20^{\text {th }}$ century that the term blend begins to acquire the meaning it has in contemporary morphology, that is, to name a word formed by fusing 
two or more words into one. For instance, Bergström (1906 § 16) considers blending "the result of an imperfect imitation, a partial analogy" that "is made up of two (or more) previously existing, generally synonymous or similar elements, each of which contributes one part to it". It is clear that Bergström's work is dedicated in the first place to speech error blends, both syntactic and lexical (though the term 'speech error blend' is not used). However, invented 'portmanteau-words' are also mentioned: "there occur some intentional or conscious ones, especially words" (1906 § 46). Bergström notes that "they are common to different languages" (1906 § 47). That is not to say that the process of blending as a way of producing new words did not exist before. Earlier examples of haplologic blends from Sanskrit, Greek, Latin and French are mentioned in Wood (1911). Nevertheless, in the $20^{\text {th }}$ century blends seem to have become a more productive way of word formation. The publication of "Through the looking glass" (1872) by Lewis Carroll catalysed the popularity of blends, and also gave rise to the term portmanteau word that is used in morphological studies either as a synonym of blend, e.g. in Pound (1967[1914]) and in Thurner (1993), or as its hyponym, denoting a type of blend, as in Algeo (1977), Piñeros (2004) or Tomaszewitz (2012). Now blend words are becoming a notable feature of contemporary language. They are often used, for example, for hybrid names (zorse $\leftarrow$ zebra + horse), or as artistic devices in headlines and other media sources (Brangelina $\leftarrow \operatorname{Br}($ ad) $[$ Pitt] + Angelina [Jolie]). Many contemporary linguists agree that blending is no longer an exceptional way of producing new words. However, the question of how exactly two or more different words can be blended into one, and to what extent the result of blending is predictable, is still open. At the root of this question is the problem of defining what kind of formations are to be classified as blends, which, as will be discussed below, have been approached by several generations of linguists in a number of ways, using various principles.

The most remarkable work of the early $20^{\text {th }}$ century dealing with blends is by Pound (1967[1914]) who gives a definition of blends, as well as their classification. Pound defines blends as "two or more words, often of cognate sense, telescoped as it were into one; as factitious conflations which retain, for a while at least, the suggestive power of their various elements" (1967[1914], p. 1). The classification given is, on the one hand, by the area of origin, on the other hand, by form. As for classification of blends depending on their origin, Pound (1967[1914], pp. 20-21) names the following types (the examples below are Pound's): 
1. Clever literary coinages, e.g. sneakret $\leftarrow$ sneak + secret and other examples from Carroll, Kipling, Wallace, Irwin, Habberton, etc.;

2. Political terms, coinages of cartoonists, editors, and other newspaper writers (Popocrat: populist + democrat);

3. Nonce blends, "originating probably in a sort of aphasia", e. g. sweedle as a result of hesitation between swindle and wheedle (1967[1914], p. 20)

4. Children's coinages, "largely accidental also", e. g. tremense $\leftarrow$ tremendous + immense (1967[1914], p. 21);

5. Conscious folk formations, "whimsical or facetous in intention and usage" (1967[1914], p. 21). e.g solamncholy, sweatspiration, bumbershoot, scandiculous, animule etc.;

6. Unconscious folk formations, "not jocular in intention but seriously meant" (Pound, 1967) e.g. diphterobia, insinuendom rasparated, needcessity, clearn etc.;

7. Coined place names or personal names, e.g. Ohiowa: Ohio + Iowa;

8. Scientific names (mainly referring to names of new chemicals), e.g. dextrose $\leftarrow$ dextrorotary + glucose;

9. Names for articles of merchandise (electrolier $\leftarrow$ electrical + chandelier $)$.

As we can see, both speech error (types 3 and 4) and creative blends (types 1, 2, 5, 6, 7, 8 and 9) are listed. Blends used as hybrid names (such as plumcot $\leftarrow$ plum + apricot) are also mentioned in Pound's work, although not included in the above classification (1967[1914], p. 18). This classification is far from being exhaustive. Moreover, Pound herself admits that "many blend-words may be classified under several of the heads suggested at the same time" (1967[1914], p. 21). She is also sceptical as to classification of blends by their form, considering that "no definite grouping seems advisable" in this respect. Nevertheless, in a later analysis of Pound's collection, Böhmerová (2010, p. 39) mentions a number of types based on formal grounds. Thus, blends can be classified according to:

- what syllable in the original word is affected by the superimposed syllable(s);

- the number of resulting syllables (admitting that monosyllabic blends cause the most difficulties in deconstructing them into source words);

- whether both elements are truncated, or only one;

- the origin of elements;

- the number of blended elements;

- the word-class of the blended elements; 
- the resulting word-class.

Pound is among the first authors who noticed the potential of blends to "achieve a permanent place in the language" (1967[1914], p. 19). Moreover, concerning the time when blends might appear, Pound (1967[1914], p. 6) observes that they "are probably as old in our language history as composites, or cross-forms, or contaminations of various kinds, in general". She supports this view by examples from Shakespeare (rebuse $\leftarrow$ rebuke + abuse), Southey (crazyologist $\leftarrow$ crazy + craniologist), Howell (foolosopher $\leftarrow$ fool + philosopher), etc.

Much stricter criteria for the definition of blends are applied by Marchand (1960, 1969). In his broad classification of English word formation types, Marchand distinguishes between 1) words formed as grammatical syntagmas, i.e. combinations of full linguistic signs, and 2) words which are not grammatical syntagmas, i.e. which are not made up of full linguistic signs. His "non-grammatical" word-formation processes include "expressive symbolism", blending, clipping, and "word-manufacturing" (Marchand, 1969: 2f). Marchand also distinguishes between speech error blends and creative blends (although the modern terms are not used in his book). From the point of view of their meaning, blends are classified into two types: blends created for expressive purposes and names for new products and scientific discoveries (1969, p. 452). This second type includes the names of chemicals, animal and plant hybrids, trade mark names etc. From the structural point of view, the category of blends as drawn by Marchand includes the so-called 'letter-words' (i.e. acronyms and abbreviations), which are considered by the majority of contemporary morphologists to be a different kind of word formation, though there are marginal cases listed, for example, in López Rúa (2004b) and Mattiello (2013) (see section 2.3 for details).

Marchand states that blending "can be considered relevant to word-formation only insofar as it is an intentional process of word-coining" (1969, p. 451). Moreover, the status of blends' constituents, according to Marchand, is different from other, more traditional, word formation units, because the constituents of blends are "morphemes only for the individual speaker who blended them, while in terms of the linguistic system as recognized by the community, they are not signs at all" (1969, p. 451). Two consequential ideas arise from this analysis: 1) that blending "has no grammatical, but a stylistic status" and 2) that "[t]he result of blending is, indeed, always a moneme, i.e. an unanalysable, simple word, not a motivated syntagma" (1969, p. 451). The idea of non- 
grammatical status of blends has been exploited by many linguists after Marchand (see section 2.3). As for the non-morphemic status of the constituents of blends, it has to be noted that some of them can eventually become morphemes (e.g. -(a)holic from workaholic, shopaholic etc.). Another idea which is often questioned is that "blending is compounding by means of curtailed words" (1969, p. 451). In fact, Marchand lists clipping compounds and blends as different types of word formation, but neither the given examples, nor the description of both types allow any exhaustive criteria for distinguishing between the two types.

In later literature, blends are not always (in fact, less and less often) perceived as a marginal phenomenon or as a grammatical anomaly. They have come all the way from being treated as "resulting in striking grammatical anomalies" (Bergström, 1906 § 47) to being considered the output of "a subconscious process assumed to be omnipresent in everyday thought and language" (Böhmerová, 2010, p. 15).

One of the first works in which blends are treated as a frequent means of word formation is Bryant (1974). The researcher compiles a list of 306 blends (251 nouns, 54 adjectives and 1 present participle) that appeared in the $20^{\text {th }}$ century, belonging to several semantic fields: fashion (60 blends); sports, travel, and entertainment (54); science and technology (44); air and space (5); home (37); political issues (15); education (3); art (7); high fidelity (13); youth (8); drug addiction (2); sex (7); health (5); 45 blends are not referred as belonging to any semantic group and thus are classified as 'miscellaneous' (1974, p. 163). Bryant (1974, pp. 163-164) made a few observations concerning the formal properties of blends (the examples below are Bryant's):

- they combine the first sounds of one word with the final sounds of another;

- in many blends some sounds are shared by both original words

- some blends incorporate a complete word as one of their elements (e.g. ambisextrous $\leftarrow$ sex + ambidextrous);

- in some blends a combining form is used as one of the incorporated elements (e.g. celebriana $\leftarrow$ celebrity + -ana);

- proper names (e.g. names of persons and names of places) can be used in blends (e.g. James Bondustry $\leftarrow$ James Bond + industry); 
Some of Bryant's examples demonstrate that such complex units as two-word proper names and abbreviations can be incorporated into a blend (e.g. James Bondustry; Max Factory $\leftarrow$ Max Factor + factory; Ziposium $\leftarrow$ ZIP [Zone Improvement Plan] + symposium).

Not all the analyses of the internal structure of blends offered by Bryant are unquestionable. Such formations as electronovision and electrofile could be classified neo-classical compounds in accordance with contemporary terminology. There are examples (acoustex $\leftarrow$ acoustic + texture; Fortran $\leftarrow$ formula translation; autodin $\leftarrow$ automatic + digital + network) that can be classified as clipping compounds or even (autodin) as acronyms. On the whole, Bryant's work is a collection of examples grouped by various features rather than a classification of blends according to any consistent criteria.

A detailed classification of blends regarding their formal structure and semantic properties is given in Adams (1973). Blends are defined as words containing splinters, i.e. "shorter substitutes" of words (1973, p. 142), which usually are "irregular in form", that is, not regular morphs. Adams mentions three major structural types of blends:

1. Words that "have to do with sound or movement of some kind" (e.g. squirl, flimmer); they cannot be easily analysed into constituents, though it is possible to state that they typically are composed by an initial consonant or consonant cluster and an ending. Elements (clusters) are called phonaesthemes (1973, p. 143), and the author admits that there can be different opinions concerning the impetus of these formations: sound symbolism, onomatopoeia, echoism, etc. (1973, p. 144);

2. Compound blends - contracted forms of compounds;

3. Group-forming (e.g. folknik, scribacious).

The closest attention is given by Adams to 'compound blends', which can be classified into several types structurally and semantically. According to their formal structure three types are distinguished: 1 ) blends of which both elements are splinters (ballute $\leftarrow$ balloon + parachute); 2) blends where only the first element is a splinter (escalift $\leftarrow$ escalator + lift); 3) blends in which only the second element is a splinter (needcessity $\leftarrow$ need + necessity). As to the semantic classification, Adams (1973, pp. 153-160) mentions the following relations between the original words that form blends: 
1. Subject $-\mathrm{V}($ screamager $\leftarrow$ screaming teenager $)$;

2. $\quad \mathrm{V}$ - object (breathalyser $\leftarrow$ breath analyser);

3. appositional of the coordinative kind (they are considered more frequent in compound blends) (brunch $\leftarrow$ breakfast + lunch , fantabulous $\leftarrow$ fantastic + fabulous, smothercate $\leftarrow$ smother + suffocate);

4. appositional, not coordinative, i.e. the first element specifies or qualifies the second (bromidiom $\leftarrow$ bromide idiom, refujews $\leftarrow$ refugee Jews);

5. instrumental (automania - mania caused by automobiles);

6. resemblance (bombphlet - pamphlet like a bomb);

7. composition (plastinaut - plastic astronaut);

8. $\quad$ synonymic (needcessity $\leftarrow$ need + necessity)

This semantic classification is largely based on the semantic classification of compounds in Adams (1973, pp. 64-89). This approach to classifying blends is justified only on assumption that the cognitive operations underlying the formation of compounds are the same for blends. It is important to note, however, that Adams herself, and many other linguists, e.g. Renner (2008) observe that coordinative relations are more typical for blends than for compounds.

The classification is revised in Adams (2001), where blending, together with backformation and shortening, is included into a bigger word-formation category of 'reanalysis', and is understood as a process that "involves the analysis of words in new ways" (2001, p. 139). Blends are defined as "made up of two contributory words, one or both of which may be only partially present in the new word" (2001, p. 139). From the point of view of origin and semantics, Adams outlines different kinds of blends depending on the extent of intentionality in their formation. Thus, three groups of blends are named: 1) unintentional blends (speech errors), that "are usually combinations of near-synonyms" (2001, p. 139); 2) deliberate blends (no formal criteria for them are provided); 3) phonaesthemic formations that occupy "an uncertain area between spontaneous errors and deliberate inventions" (2001, p. 139). Adams (2001) puts aside the definition of blends as contracted forms of compounds, but does not provide any reliable criteria for distinguishing between blends and other forms of 'reanalysis', that is acronyms and clipping compounds. 
Systematic categories of blends (both deliberate creations and lapsus linguae), outlined in accordance with Saussurean understanding of syntagmatic and paradigmatic relations, are given in Algeo (1977). All blends are classified into two main categories: syntagmatic and associative. A syntagmatic blend is defined as "a combination of two forms that occur sequentially in the speech chain", e.g. Chicagorilla $\leftarrow$ Chicago gorilla, morphonemics $\leftarrow$ morphophonemics, Amerind $\leftarrow$ American Indian. (1977, p. 56). Algeo admits that such forms are treated as blends only as a concession to traditional classifications, but notes that "a consistent taxonomy would regard them merely as contractions" (1977, p. 56). He also suggests using the term telescope words to name these formations because "it is metaphorically most appropriate for this particular kind" (1977, p. 57).

The other major category outlined by Algeo is associative blends, i.e. the ones which have "two or more etyma that have been linked in the word-maker's mind and thence in his language" (1977, p. 57). This category is subdivided into: 1) synonymic blends (e.g. swellegant, needcessity); 2) blends that combine words from the same paradigmatic class, or dvandva blends (smog) which may be also called paradigmatic; 3) jumble blends, in which "etyma are associated with one another, but not by paradigmatic equivalence" (1977, p. 58), e.g. foodoholic, dumbfound $\leftarrow d u m b+($ con)found, happenstance $\leftarrow$ happen + (circum)stance. Algeo's suggestion is to name the associative type of blends portmanteau words, to differentiate them from telescope words. The taxonomy looks very useful indeed, but the problem with this differentiation, as Algeo confirms, is that these two processes can appear either sequentially or simultaneously (1977, p. 61). An example of a combination of two kinds of blending named by Algeo is electrocution, formed as a portmanteau blend of electro- and electricution, which, in its turn, is a telescope blend of electrical and execution. Algeo (1977, p. 62) also points out that in some cases it may be unclear whether the blend is a telescope or a portmanteau, as, for example, shamateur which can be analysed as either a telescoping blend of sham amateur meaning 'one who pretends to be an amateur but is really a professional', or as a portmanteau of sham and amateur meaning 'one who tries to deceive but is amateurish'. As noted in Bauer (2012, p. 18), the problem of interpretation of blends as either having a semantic head (i.e. telescope, using Algeo's term) or coordinative (portmanteau) is of the same nature as the problem of interpreting compounds like fighter-bomber as either headed, or coordinative. 
Nevertheless, the distinction between telescope and portmanteau blends has been reconsidered by many linguists after Algeo. Some, among them Bauer (1983), Devereux (1984), Cannon (1986), include both portmanteau and telescope words in the category of blends. On the other hand, some researchers, such as Kubozono (1990), Berg (1998) and others, restrict the category to portmanteaus only, whether using this term or an alternative one, and sometimes, as in Renner (2006), subdividing them into subtler semantic categories. Revision of the distinction between telescope and portmanteau blends underlies Bauer's (2012) categories of syntagmatic origin and paradigmatic origin blends.

Apart from general systemic categories, Algeo also gives characteristics of different types of blends from the point of view of their phonological and morphological structure. In Algeo (1993), the following types of blends are named: 1) with clipped first element; 2) with clipped second element; 3) with both elements clipped (1-3 including cases with overlapping); 4) where the overlapping elements are sounds rather than words (between-ager $\leftarrow$ between + teenager; guesstimate). This structural classification implies the presence of a number of marginal cases. As noted in Algeo (1977, p. 51), “clippings are often shortened at morpheme boundaries" (e.g. betweenager - NB). In such cases, it may be hard to make the distinction "between blending and compounding under analogical influence".

Concerning the phonological structure of syntagmatic blends, Algeo (1977, pp. 56-57) mentions that the structure of blends can be the result of the phonological rules. This observation can be extended to any blends, not just of this particular type, as the influence of phonological rules on blend formation cannot be neglected, as is discussed extensively in other academic works (section 2.2). As for the phonological characteristics of associative blends, Algeo observes that in some cases such a formation originates from a set of phonaesthemes, e.g. glop $\leftarrow$ [gland, glare, glass, gloam, gloat, glub, etc. $]+[$ chop, drop, flop, plop, etc.] $(1977$, p. 60). This, on the one hand, resonates with the category of phonaesthemic blend-like formations in Adams (1973), and, on the other hand, provides another reason for considering the role of the phonological properties of the source words in blend formation and the criteria for distinguishing between some types of blends and onomatopoetical formations.

Blends as part of a general system of word formation in English are described in Bauer (1983). According to Bauer, a blend "may be defined as a new lexeme formed from parts 
of two (or possibly more) other words in such a way that there is no transparent analysis into morphs." (1983, p. 234). The criterion of analysability into morphs, called by the author "the awkward part of this definition" (1983, p. 234) is, however, crucial for the understanding of the nature of blends and may be used as one of the criteria for defining the borders of the category, however vague they might seem at this point.

Devereux (1984) compares blends with other methods of word formation in English. He mentions (1984, p. 210) two main ways of creating new words in English: 1) addition, meaning by it adding affixes to existing words to create new ones; 2) subtraction, that is, "taking letters in order from the original word or words". Subtraction is then subdivided into three subtypes:

1. Shortenings, which utilise "a group of consecutive letters contained in the original word";

2. Blends, which are "originally formed by taking the first few letters from one word and combining these with the last few letters from the other" (including the cases when either first or second word is fully preserved in the blend, e.g. pion $\leftarrow$ pi + meson; contrail $\leftarrow$ condensation trail);

3. Acronyms, which can be distinguished from blends because 1) the order of their constituents cannot be reversed (as in blends like liger $\leftarrow$ lion + tiger vs. tigon $\leftarrow$ tiger + lion); 2) acronyms are built up from the initial letter(s), unlike blends "which use terminal letters as well".

Devereux's definition of subtraction implies a focus on graphical, rather than phonological, constituents of words, which may be not relevant to the same extend to all the categories above. Devereux (1984, p. 210) compares blends to acronyms in which "the resulting concatenation of letters is pronounced as a word", unlike in initialisms such as $D D T$ where they are spelt out letter by letter. This distinction is similar to Bauer's (1983, p. 238). The distinction between blends and acronyms as defined by Devereux is not clear-cut. Bauer (1983) admits that such formations as linac $\leftarrow$ linear accelerator can be analysed either as blends or as acronyms. In later works, e.g. in Bauer (2006, 2012) such formations are labelled as clipping compounds. An important conclusion made by Devereux is that the order of words in blends, unlike acronyms, is potentially reversible. On the other hand, the constituents of many blends including 
some of the examples above (e.g. pion) cannot be reversed as they preserve the righthead structure of the underlying word combination (pi meson, i.e. a type of meson).

Two works by Cannon present an evaluation of the role of blends in English word formation and their relations to other morphological categories. In Cannon (1986), formal patterns of blending are described and analysed with regard to word formation rules, as formulated in the literature on morphology. The results of a fundamental study of English word formation from a historical perspective involving the analysis of trends and changes in the vocabulary of the English language is enunciated in Cannon (1987). All the new words and meanings, according to Cannon's taxonomy, fall within four major categories termed in Cannon (1986, p. 750) as "shifts (new meanings and functional shifts $-19.6 \%$ ), borrowings (7.5\%), shortenings (18\%), and additions (the rest)". Blends are classified in this taxonomy as a particular kind of shortening, along with abbreviations, acronyms, clippings (Cannon uses the term 'unabbreviated shortenings'), and back-formations. Blends, according to Cannon's taxonomy, are the smallest category among the shortenings (4.6\%). Yet "this category contains the most varied and perhaps the most structurally complex items" (1986, p. 750). A corpus of blends which consists of 118 noun, 11 adjective and 3 verb lexemes is analysed phonologically (with respect to overlapping sounds and syllable structure), morphologically (with a conclusion that the analysed blends come mainly from simplexes and derivations, many fewer from compounds, and one acronym), and in terms of formal structure.

Cannon observes that syllable structure is "more crucial to blending than to any other category of word formation". In particular "[t]he structure of the longer of the two source words usually dictates the maximum number of syllables, as well as the primary stress" (1986, p. 746).

From the point of view of formal structure, blends are subdivided by Cannon into two groups: 1) blends formed in such a way that both source-words share some of the letters/sounds; 2) blends which combine the first part of one word with the last part of another, but with no shared letters/sounds at the point of fusion, as in brunch $\leftarrow$ breakfast + lunch (1987, p. 144).

Concerning the semantics of various kinds of shortenings, including blends, it is stated that "our shortenings usually denote scientific subjects like chemistry and biology, though our blends primarily have commercial applications" (Cannon, 1987, p. 273). This 
latter feature of blends supports the attitude to them as to rather ephemeral formations on the marginal edge of morphology, an attitude which has been to a great extent revised in later publications about blends.

Analysing blending from the point of view of its relation to other word formation patterns, Cannon (as many other authors before and after) notes certain difficulty in separating blends from neighbouring categories. For example, he suggests that blends can be distinguished from compounds "by requiring that at least one of the two separate elements which are fused must be reduced", but at the same time he admits this distinction is "somewhat arbitrary" (1986, p. 749). In Cannon (1987) formal criteria distinguishing between various kinds of shortenings are worked out. Thus, Cannon separates blends from acronyms because in blends " $\mathrm{t}]$ he reduction usually consists of a terminal loss in the first item, plus an initial loss in the second item, where there are usually overlapping parts in the fusion" (1987, p. 144). This observation, however, is unlikely to cover all cases of blending. Finally, blends are to be separated from 'unabbreviated shortenings' because, etymologically, blends are formed from more than one item.

The category of 'unabbreviated shortenings' defined in Cannon $(1986,1987)$ covers what is elsewhere referred to as clippings (such as lab from laboratory or fridge from refridgerator), and also complex clippings or clipping compounds, exemplified by Cointelpro (Counter Intelligence Program). The latter category, according to Cannon's criteria, includes also cases like prosage which originates from protein sausage. The idea behind it is that a compound protein sausage is treated as one unit which is shortened to prosage, not as two words protein and sausage blended into one. This argumentation is hard to use for each and every case of blending because it is difficult to judge whether a multiple word unit existed in the language prior to being shortened or not. However, the idea underlying this criterion, i.e. that blends are formed from different lexical units rather than contracted from a single one, is crucial for understanding the mechanism of blending. Taking, thus, an etymological approach to the definition of blends, Cannon returns to the understanding of their nature expressed earlier by Marchand and then by Bauer: "Blending produces a new, technically simple, and often otherwise unanalyzable morpheme" (Cannon, 1987, p. 144). Later, Cannon (1989, p. 108) highlights that blends are "the only category of shortening that involves reduction of at least two preexisting 
items (the other categories involve reduction of a single source-item)". This quality of blends distinguishes them from all the other types of shortenings.

In the above classifications the formal structure of blends is mainly described in terms of categorical distinctions such as the presence/absence of shortening or overlap. It has to be noted that the studies discussed in this section are primarily descriptive, that is, a number of formal and, in some cases, semantic characteristics of blends are listed with very little or no analysis of their mutual influence. As a result, the analysis of many examples is based on arbitrary decisions, and some descriptive classifications contradict one another. The classifications of blends discussed in the following section are derived from analysing the mechanism of blend formation in relation to various factors (e.g. phonological and semantic) that may influence it. Such an analysis may provide the basis for clarifying the status of blends in the system of word formation, which is one of the objectives of the present research.

\subsection{A closer look at the mechanism of blending}

A different view on blends, compared with earlier publications, is expressed in a report on a corpus study of Japanese and English blends (Kubozono, 1990). Unlike many other researchers, Kubozono considers blending a morphological process for two reasons: simply because it is a part of word formation, and also because it "exhibits various linguistic patterns, including one relating to the notion 'head', which are common to ordinary word processes like compounding" (1990, p. 1).

Kubozono uses the term 'blend' in a narrow sense defined by Marchand (1969, p. 451) as a unit which involves "merging parts of words into one new word". Kubozono pays a lot of attention to formulating the criteria for distinguishing blending from other morphological processes. He observes that "it involves two source words in a paradigmatic relation, i.e. words that might substitute for one another, as opposed to words which occur side by side, and it is in this point that blending differs primarily from clipping and clipped compound (compound shortening), the two processes which tend to be confused with blending most often" (1990, pp. 1-2). The intention to make the syntagmatic/paradigmatic relations between source words a distinguishing criterion for different word formation patterns seems both very much justified and problematic. It is justified because it could solve a morphological problem, the solution to which has long been sought, but it is also problematic because it may not always be 
possible to tell with confidence whether the source words of a clipped compound/blend are in a paradigmatic or syntagmatic relationship, and it makes the criteria of the distinction less clear than would be desirable.

Kubozono (1990, p. 3) gives a classification of linguistic constraints on blend formation (summarised here in Figure 1), with special regard to phonological constraints that comprise the main scope of his study:

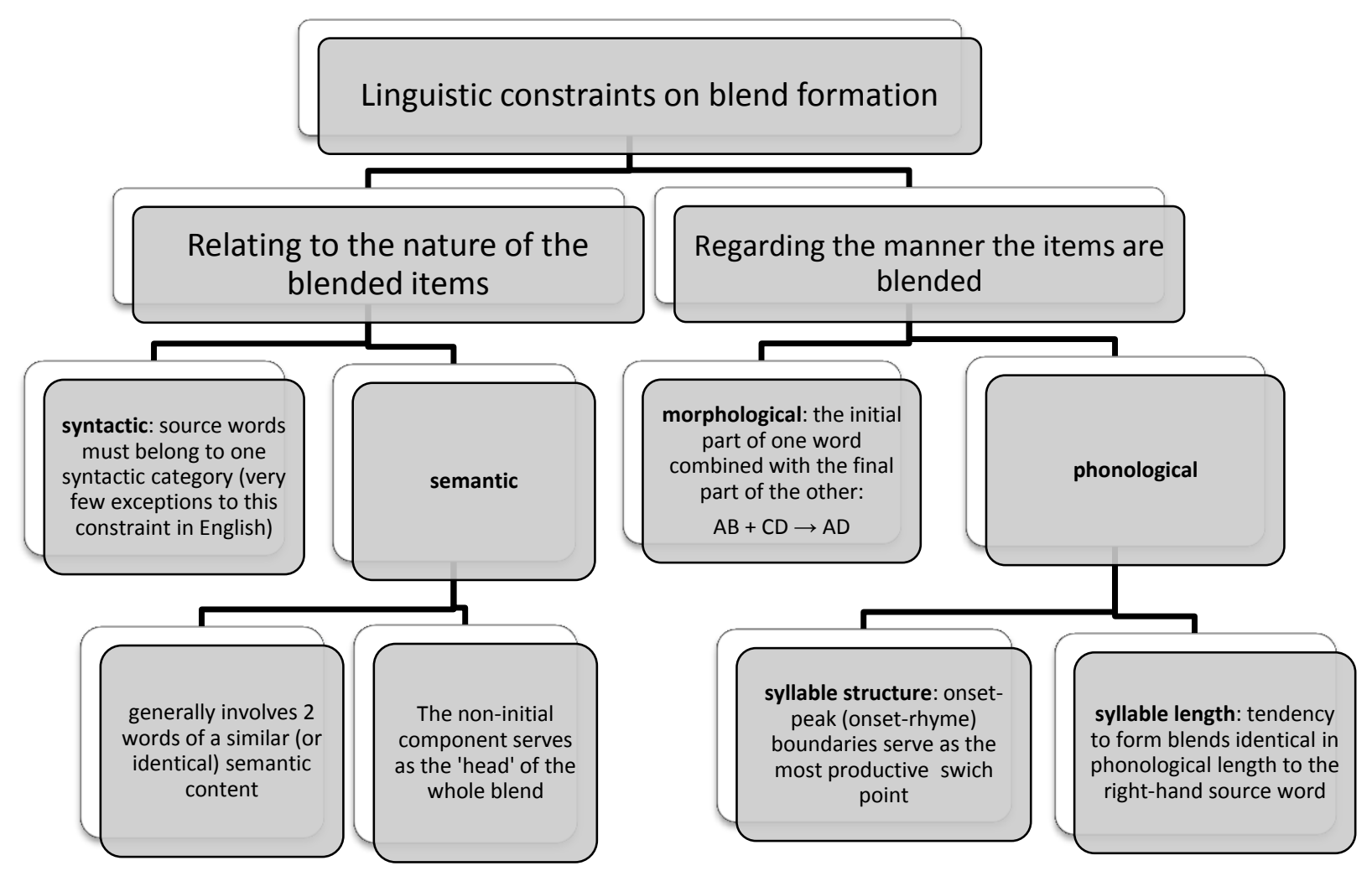

Figure 1. Linguistic constraints on blend formation.

Based on the classification in Kubozono (1990, p. 3)

The constraints mentioned by Kubozono do not work as rules that are never violated, but only express tendencies which are not without exceptions. The study of phonological constraints on blend formation is based on a collection of both unintentional and deliberate blends of English and Japanese from earlier publications on the topic. The English data are taken from Wentworth (1934), Pound (1967), and Fromkin (1973a). On analysing the corpus data, Kubozono (1990, p. 18) concludes that the mechanism of blending in English is subject to one morphological and two phonological constraints. In morphological terms, combining the initial part of one source word with the final part of the other is seen as "the most productive formation pattern" for blends (1990, p. 18), 
this observation following Bauer (1983). Two phonological constraints that condition the formation of blends are formulated by Kubozono. One constraint concerns the syllable structure of blends. It conditions the position of the switch point in blends, that is, where exactly in a blend the switch from one constituent word to the other will take place. In these terms, "the most productive switch point", as observed by Kubozono (1990, p. 18), is between onset and rime. Similar observations can be found in later works, for example, in Gries (2012), though in less categorical form, stating that the switch point is most likely to occur at the boundaries of syllabic constituents, i.e. onsets, rhymes and codas.

The other constraint is claimed to rule out "those blend forms that are not identical in phonological length to the righthand source word" (Kubozono, 1990, p. 18). The influence of the righthand source word on the length of the blend is also highlighted in Bat-El (2006) and in Gries (2004a), but as a tendency only. Morphological and phonological constraints described by Kubozono are claimed to be true not only for English blends, but also for blends in Japanese, and thus might be regarded as part of universal grammar (1990, p. 18). Though this is a very important observation as such, it is beyond the scope of the present research to draw conclusions about the universal character of any morphological rules or patterns. Nevertheless, the analysis of the phonological properties of blends performed by Kubozono, and above all his conclusions concerning the regularities governing blend formation, provided the basis for many studies of blends.

Exploring rules and regularities that underlie blend formation was one of the objectives of the research by Kelly (1998) that was based on three studies dealing with both speech error blends and intentional blends. The first study dealt with blends as "contractions of conjunctive phrases", i.e. contracted forms of and phrases, e.g. fratority $\leftarrow$ fraternity and sorority (1998, p. 580), excluding blends whose component words were drawn from different grammatical classes. The intention of this study was to predict how components are ordered in blends. "In order to examine the similarities between blend and conjunct structure" Kelly tested "whether the order of components in blends was predictable from their frequencies and syllable numbers" (1998, p. 581), and predicted "that shorter and more frequent words should be cannibalized for the first part of the blends" (1998, p. 582). As a result of the study, both predictions were shown to be supported. It was demonstrated that "words denoting prototypical members of 
categories are preferred for first position in conjuncts over words denoting less typical members" (1998, p. 583), as had been stated earlier in Kelly et al. (1986). These results can be explained by the fact that shorter and more frequent words, as well as words denoting prototypical members of categories, are accessed faster and more easily (as shown in studies on word processing discussed in Chapter 5).

Phonological properties of breakpoints in speech error blends and intentional blends were analysed by Kelly using the material from MacKay's (1982) investigation of speech error blends in English and German and of his own corpus of intentional blends. Following MacKay's finding that in German and English blend errors "the component words were generally broken at syllable boundaries" (Kelly, 1998, p. 585), Kelly extended this analysis to his corpus of intentional blends, excluding blends with overlappings, and reported that MacKay's observation turned out to be true for intentional blends as well, i.e. "the majority of breakpoints in the intentional blends occurred at word or syllable boundaries" (1998, p. 585). Kelly's corpus study shows that breakpoints in blends "cluster at major phonological joints, such as syllable, rime, and onset boundaries", which is consistent with the findings of Kubozono and other studies of the phonology of blends discussed above. Kelly explains this result by a general rule of English phonology: "The strong preference for onset-rime over body-coda breaks provides further evidence for an onset-rime representation of English syllables" (1998, p. 586).

The third study reported in Kelly's paper is dedicated to word junctures in blends. It was hypothesised that blends "tend to be arranged so that the boundary involves similar phonemes" (1998, p. 587). A consonant sonority hierarchy (Kelly uses the term 'sonorance') was worked out, in which every consonant received a rank from 1 to 7 as follows:

1 - unvoiced stops;

2 - voiced stops;

3 - unvoiced fricatives and affricates;

4 - voiced fricatives and affricates;

5 - nasals;

6 - liquids;

7 - glides. 
Kelly then uses this hierarchy to check the prediction that "the expected consonant from the first word in a blend and the supplanting consonant from the second word should come from similar places in the sonorance hierarchy" (1998, p. 587). The result of the study confirms this hypothesis, but Kelly admits that further research is needed to determine the effects of such phonological structure on the perception of blends.

In contrast to the conclusions concerning the marginal and peripheral character of blending as a word formation pattern, formulated in Adams (1973), Algeo (1977), Cannon (1986), to name just a few, Kelly's findings "demonstrate that the variability in English blend formations, though extensive, is far from random" (Kelly, 1998, p. 588). As is suggested by Kelly, insights from cognitive linguistics and cross-linguistic investigations could be helpful in checking the findings already reported, as well as in further studying various properties of blends and the mechanisms of their formation.

In a descriptive study by Jin (2005), the following characteristics of blends are analysed: 1) the length and proportions of the source words (the frequency of the source words is also analysed as a factor interconnected with their phonological features), 2) the position of the switch point, and 3) the prosodic structure of the source words. Considering the length, frequency and proportions of the source words in blends, as well as their stress patterns, Jin agrees with the findings of Cannon (1986), Kubozono (1990) and Kelly (1998) already summarised above, and focusses more on phonological considerations regarding the position of the switch point.

Jin (2005, p. 203) observes that "the majority of blends with no segment overlapping tend to split at sub-syllable boundaries: onset-rime splitting". This is different from Kelly's observation above, because Kelly's data includes overlapping blends. Analysing the data in terms of the splitting point, Jin admits that of 466 blends with sub-syllable splitting, 108 items with middle overlap are analysed as ambiguous, because it is not clear whether in twiddle $\leftarrow$ twist + fiddle, for example, the switch point is placed between $[\mathrm{tw}]$ and [1] or between [1] and [d].

Some important generalisations made in Jin (2005, pp. 219-221) are related to the syllable structure of blends and the phonological factors which regulate the order of their constituents. First, it is observed that if one of the source words does not have an onset consonant in word-initial position, it is likely to be positioned second in the blend, as in fugly $\leftarrow$ fat + ugly (2005, p. 219). Second, complex (word-initial) onset priority is 
postulated: a more complex syllable onset is preferred in the blend-initial position (2phoneme onsets prevail over 1-phoneme onsets, e.g. in droob, which Jin describes as originating from drip + boob, and 3-phonemes onsets over 2-phonemes and 1-phoneme onsets, e.g. sprig $\leftarrow$ spray + twig). Finally, lower sonority priority in the word-initial onset position is postulated: a lower sonority onset is preferable to a higher sonority one, e.g. dawk $\leftarrow$ dove + hawk $(2005$, p. 221).

Applying these generalisations to further research, it is necessary to take into account that Jin's study addresses mainly blends which combine the first part of the first source word with the second part of the second element, though some complex shortenings combining two initial parts of words (e.g. modem $\leftarrow$ modulator + demodulator) are classified by Jin as blends. Jin's study is no exception in this respect, as many researchers mention the fuzzy boundaries between blends and the neighbouring word formation categories. The principles of delimiting the category of blends and the problems with finding their place in English (and general) morphology will be discussed in more detail in section 2.3. The remainder of this section will be focussed on the research of the phonological structure of blends which, to a large extent, provide the basis for such delimitation.

An analysis of the phonological properties of blends from the point of view of different varieties of Optimality Theory (OT) is performed in three studies presented in (Renner et al. (eds.), 2012). They regard the mechanism of blending as subject to a number of violable constraints, and model the formation of blends as a hierarchy of constraints which work for a particular process (in this case blending) in a particular language. In Bat-El and Cohen (2012), a constraint-based analysis of stress in English blends is carried out. The researchers aim to explain and predict the position of stress in polysyllabic English blends (deliberately excluding clipping compounds and fully overlapping blends from the analysis). A set of faithfulness constraints is used to explain the position of stress in blends with regard to the stress and the length of the source words. This is done separately for the cases when the blend has the same number of syllables as at least one of the source words and when it has a different number of syllables. To account for the situation when the right-hand source word is a monosyllable and for the fact that, despite the expected right-hand stress on this element, a blend acquires the default word stress, the authors "assume that 
monosyllabic words are not lexically stressed" (2012, p. 207), and the model based on this assumption successfully explains nearly all the observed cases.

'Output-to-output faithfulness in the phonological structure of English blends' is discussed in Tomaszewicz (2012). The study explains the phonological structure of blends using the concept of a prosodic word, that is, a phonological constituent larger than foot but smaller than phonological phrase. An important feature of prosodic word, as described in Hall (1999, p. 2) is that it "must align with morpho(syntactic) boundaries". This implies that a monomorphemic word cannot contain more than one prosodic word, unlike, for example, compounds, each constituent of which corresponds to a prosodic word. In Tomaszewicz (2012), the regular patterns in the phonological structure of blends are viewed as the output of the process of mapping the phonological material of two source words onto a template of a single Prosodic word. The main finding of the study is that metrical well-formedness and constraints on word-internal phonotactics are crucial factors which determine the output of the blending process.

A similar finding is reported in Trommer and Zimmermann (2012), presenting an OT analysis of a specific type of Spanish blends referred to as portmanteaus in the sense of Piñeros (2004). Following the analysis of English truncations in Lappe (2007) as mapping the phonological material of a word onto the template of a Minimal Prosodic Word (that is, the prosodic word of a minimal size allowed by the phonology of the language), Trommer and Zimmermann explain blending as mapping the phonological material of one source word onto the prosodic template of another by substituting the required segments. Their model, though, applies only to a specific type of blends answering its criteria, which makes the main argument circular.

The problem with an OT analysis of blending is that, given the variability of the form of blends, the set of constraints which successfully explains the formation of one structure is not suitable for explaining the formation of another type of blends. As a result, each particular set of constraints is used to successfully explain the formation of blends answering a pre-selected set of structural criteria, which leaves the question of why a particular set of criteria was chosen unanswered.

A possible way of avoiding the problems described above is to make taxonomically relevant generalisations relying on the information provided by the analysis of the lexical data. This is the approach adopted in the present research. In this respect, this 
research largely builds up on a corpus study of the formation of blends by Gries (2004a; 2004b; 2004c; 2012). The definition of blends used in Gries (2004a, p. 416) specifically distinguishes intentional blends from speech error blends:

Blending is the intentional coinage of a new word by fusing parts of at least two source words. Usually, at least, the fore part of the first source word ( $\mathrm{sw}_{1}$ ) is combined with the hind part of the second source word (sw2) and there is some phonemic or graphemic overlap of the source words

The definition proposed by Gries also implies that blends are different from clipping compounds which are understood as consisting of clippings of initial parts of the source words. This assumption is further tested in Chapter 4 of the present thesis using a set of methods different from those used by Gries.

Among the blends that fall into the scope of his study, Gries (2004a, p. 415)

distinguishes the following types, regarding their formal structure (in the examples below, as well as further in the thesis, the parts of the source words that are not retained in blends are in parentheses, the overlapping segments are in bold type):

1. the source words do not overlap in the resulting blend: brunch $\leftarrow$ br(eakfast) + (l)unch;

2. the source words overlap: motel $\leftarrow \operatorname{mot}($ or $)+(h) o t e l$;

3. the source words overlap, and the first word is entirely present in the blend: foolosopher $\leftarrow$ fool + (phi)losopher;

4. the source words overlap, and the second word is entirely present in the blend: austern $\leftarrow \operatorname{auster}(e)+$ stern;

5. the source words overlap so that both words are entirely present in the blend: alcoholiday $\leftarrow$ alcohol + holiday.

Giving a suitable taxonomy of blends is not the ultimate aim postulated by Gries. Claiming that the majority of the previous "studies on blending are mainly taxonomic in nature [...] and contribute little to the explanation of why blends have the structure they have" (2004a, p. 416), the researcher expresses the intention to provide this explanation. Instead of attempting to provide evidence for a categorical distinction between blends and other types of word formation, Gries adopts a probabilistic 
approach, which he considers more apt to such diverse phenomenon as blends. In Gries (2004b, p. 645) a profound analysis of previous definitions and classifications of blends is given. The analysis demonstrates, on the one hand, that blends are incredibly various, and on the other hand, that the studies of blends also vary in terms of parameters they use to define and classify blends. Gries (2004b, p. 645) then concludes that most of the studies provide "purely structural definitions" which "need to be taken with a grain of salt, given that many criteria are not absolute".

Among the studies that are not purely taxonomic, Gries names the above cited works (Kelly, 1998; Kubozono, 1990) that take into consideration the similarity of the source words to each other and their similarity to the blend (2004a, p. 416). Both these factors are interpreted by Gries as related to 'stages' of creating a blend by an active subject (2004, p. 426), although the 'stages' are, as Gries later (2012, p. 147) admits, not to be treated as "isomorphic to the actual psycholinguistic processes". The stages are: 1) choosing the source words to be blended, and 2) merging the source words into a blend. On the first stage the blend coiner chooses to blend source words that not only have the necessary semantic qualities to fulfil the communicative intention of the blend coiner, but "are similar to each other in terms of letters, phonemes and stress patterns" (2004, p. 427).

On the second stage of blend creation, the source words are blended in such a way that 1) they are still recognisable, and 2) "the resulting blend is still sufficiently similar to both source words in terms of letters, phonemes, length, and stress pattern" (2004, p. 427). Gries compared a collection of 988 blends to a corpus of simulated blends created from the same source words (that is, for each real blend a cluster of simulated blends was generated, combining various parts of their source words). The real blends were compared to the simulated blends in two aspects: 1) the degree of preservation of the source words in the blends, i.e. number of letters and phonemes of the blended words included in the blends, and 2) the degree of similarity between the blends and their source words. The comparison between showed that real blends preserved more material from their source words, and also were more similar to their source words, than random simulated blends.

It appears that two opposed factors interact in blending: on the one hand, maximisation of the degree of recognisability of the source words, on the other hand, the desire of the blend coiner to maximise similarity. The conclusions about the 'desire' of blend coiners 
are, of course, assumptions only, as they are made on the basis of corpus research, rather than received in any form from blend creators directly. It does not mean that the conclusions are wrong, but different sets of factors included in corpus analysis may reveal different regularities and thus lead to different conclusions about the formation of blends. This is something to be kept in mind, and because of this it is important to check the assumptions in a study involving human participants.

Two other publications by Gries (2004b, 2004c) present two case studies analysing recognisability and similarity of source words in blends. The aims of the studies are closely related to the problematic questions outlined in (2004a). To be precise, Gries aims to investigate the reasons why intentional blends have the structure they have, and also to compare them to speech error blends in order to define to what degree they are similar to each other.

Concerning the degree of recognisability of the source words, the case study based on a corpus of 988 intentional blends plus 90 authentic speech-error blends and 34 experimentally-induced speech-error blends demonstrated two characteristic patterns:

1. shorter source words contribute more of their material to the resulting blends (in terms of the percentage of graphemes / phonemes of the source words retained in the blend);

2. more material of the final (in terms of the order of presence in the blend) source word tends to be retained in the blend than of the initial source word.

These results support the hypothesis of Kaunisto (2000), who, following Bergström (1906), argues that the deletion of any part of the source words presents a threat to the understandability of the blend. Therefore, Kaunisto concludes that ideal blends would be "ones where the ending of the first source word and the beginning of the second source one overlap, resulting in a way in no deletion at all". Moreover, according to Gries (2004a, p. 4), the recognisability of the second word is reduced because it loses its beginning in blending, and therefore "is not processed in the normal way". To compensate for this, a greater portion of the second source word than of the first one has to be preserved.

The findings of the study demonstrate that the recognisability of source words influences the structure of intentional blends, and provide criteria for the distinction 
between speech-error blends and intentional blends. In particular, intentional blends, unlike speech error blends, tend to maximize the recognisability of their source words and exhibit markedly distinct lengths and frequencies of the source words. However, the degree of similarity of the source words to each other and also to the blend is similar in speech error and intentional blends.

Considering the length and frequency of the source words, the following differences between intentional and speech error blends are revealed by Gries: the average frequencies of source words of error blends differ significantly such that the second source word tends to be much more frequent than the first one. As to intentional blends, the significant effect is in fact in the opposite direction, that is, the more frequent source word usually comes first in the blend (cf. Kelly, 1998). As to the similarity between the source words, the results described above are compatible with an important finding by Kubozono (1990) that the source words of blends are significantly more similar to each other than random words of approximately the same word classes.

The results of these studies were later re-analysed taking into consideration a corpusbased investigation of various kinds of 'subtractive word-formation' (Gries, 2006), extending the previous studies by including in the scope of the work comparative analysis of intentional blends, speech error blends, and clipping compounds, or, to use Gries' term, 'complex clippings'. The results of the studies and the theoretical implications of them are summarised in Gries (2012), where intentional blending is analysed regarding 1) the degree of similarity between the source words, 2) the ordering of source words in blends, and 3) the position of the switch point. These aspects of intentional blending are viewed by Gries as three interrelated temporal stages: 1) the selection of source words; 2 ) the decision for a particular order of the source words in the blend; 3 ) the "decision of how exactly to split up the words and blend them" (2012, p. 146).

As one way of comparing intentional blends, error blends and clipping compounds in terms of the choice of the source words, Gries used the similarity of the source words and the resulting formations (measured in graphemes, phonemes, and graphemic and phonemic $n$-grams). The analysis showed that the source words of error blends are more similar to each other, as well as to the blend, than those of intentional blends. The source words of intentional blends were proved to be on average more similar to each other and to the blend than the source words of clipping compounds. Moreover, the 
qualitative differences in similarity were shown. In particular, the locus of similarity in intentional blends is concentrated around the middle and end of the blend, unlike in speech error blends where it spreads across the word. In clipping compounds, the locus of similarity was found to be concentrated at the beginning of the word.

The selection of the source words to blends, according to Gries, tends also to be subject to their semantics. On analysing these types of relations in different kinds of blending and in complex clippings, Gries comes to the conclusion that the source words of error blends tend to be synonyms (e.g. need and necessity), while the source words of intentional blends can be involved in various semantic relationships, synonymy being only one of them. Such difference can be explained in terms of production processes. Experimental studies of word production have shown that accessing a word from one's mental lexicon can involve simultaneous activation of other words which are phonologically and/or graphically similar to it (see, for example, Marslen-Wilson (1987), and also the discussion in Chapter 5). The source words of clipping compounds, unlike those of blends, show a tendency towards expressing 'contractive relations' (2012, p. 155 ) , that is, are likely to be adjacent in a compound (e.g. scifi $\leftarrow$ science fiction).

In terms of the ordering of the source words, Gries (2012, p. 158) observes the following differences between intentional and error blends: the second source word of intentional blends tends to be longer than the first one, and also is less frequent, which supports earlier findings reported, for example, in Kelly (1998).

Gries pays a lot of attention to analysing the particular ways in which the source words are blended, that is to the choice of the switch point and the amount of each source word preserved in the blend. The placement of the switch point is related to the similarity and its location in the source words, and also to recognisability of the source words. In relation to the latter, Gries (2006) introduces the notion of 'selection point' which is the point marking the border of a particular segment in a given word after which the word is the most frequent word with that segment (Gries used the British National Corpus to extract frequencies). The notion of selection point is closely related to the notion of 'uniqueness point' elaborated by Marslen-Wilson and his colleagues in the context of the Cohort model of word recognition (Marslen-Wilson 1987; Tyler 1984). According to this model, as soon as the beginning of a word is heard, all the words that start with the same sequence of sounds become activated in the hearer's mental lexicon, and this set of words is referred to as 'word-initial cohort'. The process of word recognition and the 28 
research on it will be discussed in more detail in Chapter 5 . What is relevant here is that for any given word there is a certain point after which there are no other words which start with exactly the same set of sounds. The location of this point is correlated with that of the psycholinguistically relevant 'recognition point' defined in Marslen-Wilson (1987, p. 80) as 'the point at which, starting from word-onset, a word can be discriminated from the other members of its word-initial cohort'. Gries relates his selection point to the recognition point as well, and his findings show that the source words of the intentional blends are split up in such a way as to facilitate their recognisability (that is, the first source word tends to be split up "nearly exactly at the selection point", and the second source word is split up on average "half a phoneme too early" (Gries, 2012, p. 162). In contrast, complex clippings tend to split up much earlier, and thus to preserve much less of their source words than would be optimal for their recognisability. These findings provide reliable criteria for distinguishing between clipping compounds and blends and can be used as basis for further study of these categories including other factors and methods.

\subsection{Grammatical or extragrammatical?}

The research findings overviewed in the previous section show that the amount of knowledge about blending, and also the systematicity of this knowledge, has increased dramatically in the last few decades. It is not surprising, therefore, that blends are less and less often considered a marginal phenomenon in word formation, or at least the notion of marginality in morphology has undergone a notable change in meaning. For example, in Dressler (2000) blends are regarded as a phenomenon of extragrammatical morphology (as opposed to marginal morphology), together with other 'extragrammatical morphological operations' such as abbreviation and reduplication. Classifying these types of word formation as extragrammatical does not imply putting them outside the scope of morphological studies, but highlights the importance of a better understanding of them and the qualitative differences between them and "the prototypical cores of morphological grammar" $(2000$, p. 8).

Similar theoretical assumptions underlie the work by Mattiello (2013) which aims to draw a distinction between extragrammatical phenomena and the regular morphological grammar (2013, p. 4), and to provide systematic analysis of the phenomena of English word formation which the author classifies as extragrammatical, i.e. abbreviations, blends, reduplications, back-formations, infixations and 
phonaesthemes. Mattiello highlights that extragrammatical phenomena should not be analysed in the same way and according to the same principles as regular morphological grammar, or "marginal morphology" as defined in Dressler (2000), or "expressive morphology" as defined in Zwicky and Pullum (1987). Mattiello thus opposes the claim made in Bat-El (2000) and Plag (2003) that such formations, and blends in particular "are highly systematic in nature and should therefore not be excluded from what has been called 'grammatical morphology'” (Plag, 2003, pp. 125-126).

A detailed classification of blends provided by Mattiello is based on three perspectives, as follows:

1. Morphotactically (this term is exploited by the author), all blends are classified into 'total', that is, those in which both the source words are reduced, as in ballute $\leftarrow$ ball(oon) + (parach)ute, and 'partial', "in which only one source word is reduced" (2013, p. 120), as in floordrobe $\leftarrow$ floor $+(w a) r d r o b e$. It has to be noted that blends in which both source words are preserved intact, receive no label under this classification.

2. Morphonologically and graphically, Mattiello classifies blends into non-overlapping and overlapping, with more fine-grained subdivision of the latter into several types depending on type of overlap (both graphical and phonological, as in mousewife $\leftarrow$ mouse + housewife, only phonological, as in cartune $\leftarrow$ cartoon + tune, or only graphical, as in smog $\leftarrow$ smoke + fog $)(2013$, pp. 122-123).

3. Semantically, Mattiello differentiates between attributive blends, e.g. Fruitopia $\leftarrow$ fruit + utopia , and coordinative blends, e.g. chemagination $\leftarrow$ chemistry + imagination The first type in other classifications is termed as 'syntagmatic origin' blends (Bauer, 2012) or 'telescope' blends (Algeo, 1977). The second type is what is elsewhere referred to as 'paradigmatic origin' blends (Bauer, 2012) or 'portmanteau' blends (Algeo, 1977).

On the one hand, Mattiello provides a scrupulous classification of a rich collection of multiform examples, and a meticulous analysis of the well-formedness of diversified phenomena. On the other hand, the criteria of classification used by Mattiello for blends and other phenomena such as clippings are not always used consistently. For example, clipping compounds are analysed as 'back-clippings' only when uninterrupted part of a compound is retained, as in prefab $\leftarrow$ prefabricated structure, while formations retaining the initial parts of both compound constituents (e.g. biopic $\leftarrow$ biographical picture) are classified as 'random clippings' alongside with cases like $H R N \leftarrow$ heroine (Mattiello, 30 
2013, p. 82). At the same time, examples like agitprop $\leftarrow$ agitation + propaganda (p. 113) are assigned to the category of blends (2013, p. 113).

Examples of many different patterns of blends and clippings are given to support the view that these formations are 'variable and minimally predictable' (2013, p. 96). It has to be noted, though, that each of the 'regularities' of blends is analysed in isolation, and the possibility of interaction of different constraints is not considered (cf., for example, the violation of the length constraint in psychedelicatessen $\leftarrow$ psychedelic + delicatessen, which may be the result of high degree of preservation of the material from both the source words).

One of the key claims made by Mattiello is to oppose such scholars as Bat-El (2000) and Plag (2003) who admit a considerable degree of predictability of clippings, blends and related phenomena. To support the argument that such formations 'are extragrammatical in nature, and regulated, at most, by analogical processes' (Mattiello, 2013, p. 253) a lot of counterexamples to generalisations made in Bat-El (2000) are offered. It is concluded that the output of all extragrammatical operations is unpredictable or only partially predictable. However, if the prototypicality and probability of the observed patterns is included in the analysis, as, for example, in Bod et al. (2003), it is possible to develop an approach which stretches the boundaries of the 'regular' morphology and includes both 'regular' phenomena and those which are treaded by Mattiello as extragrammatical in a unified picture. A number of studies that provide successful attempts to predict the output of extragrammatical formations using prosodic and morphophonological characteristics are not taken into consideration by Mattiello, although the results reported there can show that the distinction between 'grammatical' and 'extragrammatical' may not be a categorical one. Rather, if the regularity of blend formation is analysed in terms of tendencies, which are realised with greater or lesser frequency (as was shown, for example in Gries (2012), Arndt-Lappe and Plag (2013), and other studies discussed in section 2.2), it is possible to describe them in terms of regular morphological constraints. What is not clear to date is the status of blends in morphological taxonomy, which is envisaged in the following section.

\subsection{The position of blends among the neighbouring morphological categories}

As it proves impractical to define and describe blends in terms of categorical distinctions, another approach is to work out certain criteria of their well-formedness. 
A prototypical approach to classification of word formation categories involving some degree of shortening (including blends) is suggested by López Rúa (2002, 2004a, $2004 \mathrm{~b})$. The main purpose of the research reported in $(2002,2004 \mathrm{a})$ is the analysis of acronyms and alphabetisms, and blends as well as clippings are included in the scope of the work as neighbouring categories to initialisms (2002, p. 33). It is necessary to add that the understanding of what needs to be included in the category of blends, as expressed by López Rúa, does not agree with most classifications (see the description of prototypical and peripheral blends below). Nevertheless, the approach itself can provide a lot of insights, as it differs radically from classical approach to building taxonomies, as can be seen from the following (2002, p. 34):

I suggest that a comprehensive view of the categories under study can be achieved by resorting to the radial polycentric model of categorial description: while respecting the particularities of all the categories involved, this structure efficiently represents the smooth transitions from the centres to the peripheries of those categories, and provides a unifying link which subsumes them as instantiations of the superordinate category of shortenings.

López Rúa analysed a corpus of 9,600 items (among them 7,848 initialisms, 1,100 abbreviations, 195 clippings, and 457 blends) according to a number of parameters (2002, p. 35):

1. number of source forms (one or more) and type of 'the morphosyntactic unit' which is shortened (word or phrase);

2. pronunciation of the resulting form (pronounced as a word or letter by letter);

3. orthography (small letters, capitals, or a combination of both);

4. degree of shortening: from maximum (one or two initials replacing one source word, as in laser), to medium or minimum, in which the resulting forms retain splinters (brunch) or even complete words (e.g. bank in Eximbank $\leftarrow$ Export Import Bank) of the source;

5. degree of phonic integration of the constituents: high (sound intersection or overlap, as in motel), medium (the adjacent sounds from the parts of the sources are joined to form a syllable, as in brunch, or a "pronounceable sequence", as in 
radar), or low (each original constituent provides "an independent syllable", as in Nabisco $\leftarrow$ National Biscuit Company);

6. mode of expression (speaking and writing, or only writing).

On applying these parameters to the items in the corpus, López Rúa then outlines the prototypical characteristics of the categories under consideration. Blends are described (2002, p. 41) as typically formed of two words, written in small letters, read as a word, and used both in oral and in written speech. Prototypical blends are characterised by medium degree of shortening, and high to medium degree of phonic integration (meaning that they may or may not have phonological overlap). Using the same characteristics, López Rúa names less prototypical, or peripheral cases of blends (2002, p. 46):

- the borderline cases between acronyms and blends, e.g. Codesh: 'COnvention for a DEmocratic SoutH Africa', Cospar/COSPAR: 'COmmittee on SPAce Research';

- blends retaining complete words or combining forms as constituents, e.g. robomb $\leftarrow \operatorname{robo}(t)+$ bomb, minex $\leftarrow$ mine (warfare) ex(ercise), slanguage $\leftarrow$ slang + language; these cases are considered to "lie closer to proper compounds";

- letter compounds (also called 'semi-abbreviations'), e.g. e-mail (e: `electronic'), PT boat (PT: 'Patrol Torpedo');

- clipped compounds, in which the constituents are "initial splinters joined with a low degree of phonic integration", e,g, Alcan $\leftarrow$ Al(aska) + Can(ada)

- clitics (didn't, I'll) which "could also be regarded as a very marginal type of blends with some features of clippings".

Still less prototypical cases than ones named above are regarded as hybrids: acronymsalphabetisms-blends, abbreviations-blends, or clippings-blends (terms used by López Rúa).

The results of the analysis are presented in the form of radial polycentric model (2002, p. 55), in which the five categories under consideration (clippings, blends, abbreviations, alphabetisms and acronyms) are represented as apexes of a pentagon, with bilateral connections between all categories with areas of borderline and hybrid cases marking each of them. 
The question that arises with regard to this classification is why the categories under consideration are to be unified in one categorial continuum. In (López Rúa, 2004a, p. 123) an explanation for such approach is that "basically, they are all instances of complex shortening".

Thus, López Rúa (2004a, p. 124) regards shortening as "a superordinate word-formation device, typically consisting in the reduction of old bases (one or more)". The researcher distinguishes between simple and complex shortening, simple shortening involving "exclusively graphic", and complex shortening involving both graphic and phonic reductions. Thus, simple and compound abbreviations are regarded as simple shortenings, and complex shortenings is said to include clippings, blends and initialisms (the latter categories involving different degrees of reduction).

A closer look at blends and their relations to the neighbouring categories is taken in (López Rúa, 2004b), where not only shortenings of various kinds are considered, but also complex lexemes that are formed from more than one source word/root/bound morph without shortening, i.e. compounds. The author "intended to establish a gradual progression which could connect all the categories in a uniform and consistent way, while providing a coherent account of troublesome irregularities (peripheral cases and hybrids) commonly left aside" (2004b, p. 76). The 'categorial continuum' is, thus, built as follows, taking into consideration the six parameters stated above and placing different kinds of complex lexemes on one line from full presence of all the constituents of a complex lexeme to the lowest degree of their presence (2004b, pp. 74-76).

1. compounds;

2. neo-classical compounds;

3. blends where the source words overlap and are retained in their entirety;

4. blends that consist of "clusterings, unions or intersections of splinters and stems or combining forms" (2004b, p. 74);

5. "items only composed of splinters, thus involving a higher degree of shortening than the previous group and also different degrees of phonic integration" (2004b, p. 74);

6. 'letter compounds' (2004b, p. 74);

7. peripheral acronyms. 
This continuum can then be logically finished by acronyms and abbreviations. A similar conclusion is drawn in Bauer (1998), on the basis of a study of neoclassical compounds. In particular, Bauer (1998, p. 414) considers blends and clipping compounds as 'intermediate stages' between compounds and abbreviations in terms of the degree of shortening involved in their formation.

In the categorial continuum described by López Rúa, all lexemes which include parts of other lexemes in this or that form are called blends, and clipped compounds are treated as a marginal subcategory of blends. The placement of certain examples in the continuum is unclear: many examples listed as blends can be classified as acronyms or clipped compounds, even using the same six parameters but with slightly different interpretation. If it is only a matter of terminological difference, the exact term may not be important as long as the researcher provides definitions. But there may be semantic, functional and/or processual differences between blends, clipped compounds and acronyms, and if there are such, they will help to draw the borderlines (though they may be fuzzy as well) between these lexeme classes. López Rúa (2004b, p. 67) admits that there may be other criteria for analysis that were not considered in her study, to be precise, "two other characteristics may be of help in the organization of blends, namely word-stress and what Marchand (1969) calls 'the psychological unity' of the combination, that is, the degree of semantic integration of the constituents" [my italics NB]. To this one can add that not only word stress, but a number of phonological characteristics should be taken into consideration.

In Fradin (2000) blending is analysed together with other unorthodox word-formation processes "for which it is difficult to know, at first glance, whether they belong to morphology proper" (2000, p. 11), i.e. word formation using various kinds of combining forms. Fradin's classification of combining forms is a development of the one by Warren (1990, p. 116) which includes the following groups:

1. Allomorphs of model words, e.g. astro- from Lat. astrum; -drome from Gk. dromos - these forms are often called neo-classical and can occupy either initial or final position in the word (as stated in Bauer (1983), often the same combining forms can be used in different positions).

2. Truncated forms of model words, e.g. cyber-from cybernetics, -(a)holic from alcoholic - these forms can also be both initial and final. 
3. Parts of model words, which happen to be established morpheme-forms, e.g. -gate from Watergate - these forms are initially parts of blends, only final parts tend to become morpheme-forms.

The combining forms described by Warren may be formed in two different ways:

1. Phonetic modification of an existing morpheme: 1) minor modification, as in case of neo-classical combining forms, or in case of adding a linking vowel; 2) clipping.

2. Secretion, which "is not simply an abbreviation process but is seen as a process which makes it possible to create new morphemes" (B. Warren, 1990, p. 125), i.e. involves not only shortening of the form, but also discarding part of the meaning or creating new meanings.

A certain regularity reported by Warren, as later observed in Fradin (2000, p. 20), is that first elements of complex formations such as ecosocialism, Eurofighter are abbreviated forms, while second elements in spendaholic and bikeathon are secreted forms.

Fradin reconsidered this classification on analysing a corpus of French and English examples with a focus on their semantics and also taking into consideration their phonological properties. The heading of the combining forms is classified in Fradin (2000, p. 53) into four types:

1. Learned word formation, that is, the formation of new learned forms using bound morphs or affixes of heterolexical origin (e.g. bathyscaphe, hydrure); Fradin calls them 'classical combining forms', and the formatives using bound roots of this kind are often referred to as 'neo-classical compounds' (e.g. hydrophobe).

2. Blending, in which the formatives "are abbreviated lexemes whose meaning combine in the same way as in compounding" (e.g. camcorder, motel).

3. Secreted affixing, which is a combination of a lexeme or an abbreviated lexeme with a 'secreted affix', i.e. a part of a lexeme that has undergone secretion (e.g. workaholic, Irangate, vodkatini).

4. Concealed compounding, that is, formation of a new lexeme out of an abbreviated form of a model lexeme (also called fracto-morpheme) and another lexeme in full or abbreviated form (e.g. éducatique, télévente, freeware, shareware). 
Judging only by the formal representation, it is difficult or even impossible to distinguish between lexemes in groups 2-4, all of them being referred to as blends in various sources. The core difference, according to Fradin (2000, p. 46), lies in the semantics, and is based on the nature of the process that the part of the lexeme undergoes on one of the stages of word formation. The processes are: 1) abbreviation, which is understood as "shortening phonological representation of a lexeme", but preserving the meaning of it; 2) semantic selection - "shortening or sorting out semantic representation of a lexeme"; and 3) abstraction - "abstracting over a predicate inside the semantic representation of a lexeme" (2000, p. 53). It is stated that only if the semantic component of the source lexemes is preserved in the new form, this new form deserves to be called a blend, otherwise it is a different extragrammatical process. In diachronic perspective this agrees with the observation that the constituents of blends may eventually become combining forms (cf. Lehrer (1996), see also Chapter 5), but the semantic processes that must take place in order to transform one kind of extragrammatical formation (in the terminology used by Fradin) to another, can be very hard to track down. In addition to this, Fradin's classification implies that in terms of semantic relations between constituents all kinds of word formation considered, except learned affixation, are similar to compounding, but this may not always be so.

The idea of marginality of lexical blending is maintained in Brdar-Szabó and Brdar (2008), presented in the light of contemporary cognitive linguistic studies. Following Kemmer (2003), Brdar-Szabó and Brdar recognize lexical blending as an instance of conceptual integration, "just one of the many ways in which conceptual integration or blending may manifest itself" (2008, p. 172). The central question of their study is why, notwithstanding the fact that conceptual integration is claimed to be one of the central cognitive processes, lexical blending should be marginal and even "almost non-existent as a word formation process in certain languages". In order to answer this question, they study phonological and structural properties of blends and neighbouring categories (compounds and clippings) in a cross-linguistic perspective, on the material of English, German, Hungaran and Croatian.

As many other linguists, Brdar-Szabó and Brdar (2008, p. 175) confirm that the study of the blending is impossible without taking into consideration other methods of word formation, such as clipping and compounding, because "we are in fact dealing with a 
cluster of phenomena exhibiting family resemblance". Semantic and phonological criteria are used to build the classification (2008, p. 175), which includes:

1. The core items that should be treated as the most prototypical blends (though the term 'prototypical' is not used). They are the lexemes formed so that "the input words are shortened in their seam, i.e. the end of the left-hand item and the initial segment of the right-hand item, or/and they share a phonological segment", and semantically "they are co-hyponyms of some third item", e.g. goabex $\leftarrow$ goat + ibex, magalog $\leftarrow$ magazine + catalog, prosumer $\leftarrow$ producer + consumer.

2. Determinative compound-like blends, the input words of which fail to qualify as co-hyponyms, e.g. spam $\leftarrow$ spiced + ham, warphan $\leftarrow$ war + orphan.

3. Clipping compounds, the elements of which are in a determinative relationship and the resultant formations do not exhibit phonological overlap.

The items in the first category are described as typical blends because "they often exhibit diagrammatic iconicity in the sense that semantic overlap tends to be accompanied by phonological overlap" (2008, p. 175).

It should be noted that this classification is presented as a suggestion rather than a formal taxonomy, and both the criteria could be questioned. Firstly, the semantic relations between the input words cannot always be defined unmistakably. Secondly, the criterion of having/not having a phonological overlap may not be sufficient in itself. It is not known whether the factors of semantic closeness and phonological resemblance are really connected, and if they are, whether the process of blending is really governed by semantic closeness or is it more determined by phonological likeness.

According to Brdar-Szabó and Brdar (2008), compounding and clipping are not only neighbouring word formation phenomena to blends, but also prerequisites for the productivity/marginality of blending. In other words, the degree of productivity of compounding and clipping in a certain language determines the status of blending in the word formation of this language. The authors claim that the productivity of the three word formation processes under consideration is closely connected, and illustrate this argument by the example of four European languages as follows (2008, pp. 190-191): "Croatian exhibits very little compounding and only peculiar types of clipping and also has very few blends. English seems to be on the other pole of the productivity 
continuum on all three counts. [...] German and Hungarian pattern somewhere in the middle". The findings of Brdar-Szabó and Brdar lead to further questions to be dealt with in morphological research. Needless to say, it is important to find out if the patterns outlined in their study work in other languages. However, the comparative study of this kind is beyond the scope of the present research. Focussing particularly on English, one might need to reconsider the interconnections between phonological and semantic relations of the source words in blends and other related categories.

A view of blends in a broader range of related categories is taken in Fandrych $(2004$, $2008 b)$. The study focusses on non-morphematic word-formation defined as "any wordformation process that is not morpheme-based [...], that is, which uses at least one element which is not a morpheme; this element can be a splinter, a phonæstheme, part of a syllable, an initial letter, a number or a letter used as a symbol" (Fandrych, 2004, p. 18, emphasis in the original). Thus, non-morphematic word formation includes: 1) shortenings: acronyms, blends and clippings; 2) onomatopoeia. Fandrych considers it unsuitable to analyse the three word formation categories as sub-categories of each other, supporting this point of view by the observation that each word formation category involves different "submorphemic concepts": initials form acronyms, (bound) splinters form blends, and 'free splinters' (the term used by Fandrych in analogy to 'free morphemes') form clippings (2008b, p. 117). One of the key differences between blends and clippings, according to Fandrych, is that the former contain bound splinters, while the latter are formed by 'free splinters', e.g. the clipping blog, which is not equal to any of the original morphemes of weblog, is classified as a 'free splinter' which, eventually, acquires a status of a free morpheme. Although no clear criteria of distinguishing between free and bound splinters are suggested in Fandrych $(2004,2008)$, the distinction itself is important for the present research, as one of the criteria of data sampling to be elaborated.

Studying blends in the surrounding of the neighbouring morphological categories may be more fruitful than focusing on a category built in the limits drawn according to a set of arbitrary criteria, but it implies its own hidden dangers. That is, the choice of criteria used to define typicality / marginality of data, has to be chosen on certain grounds, which can be very different in different studies. An example of the analysis of blends relying on a set of criteria which are essentially different from those mentioned above is 
Renner (2006). The following set of "yes/no" criteria is used for defining the typicality of blends:

- double truncation

- internal truncation

- interpenetration (the term used by Renner for overlap)

- coordination (2006, p. 134)

Including coordination as a criterion of typicality is in accordance with the overall scope of Renner's research which aims to analyse coordinative relations in different kinds of complex lexemes in English and covers blends as only one type of such lexemes (Renner includes clipping compounds in this category). Renner (2006, pp. 158-159) subdivides the coordinative relations between the source words into the following types:

- hybrid relations: 58\% (e.g. ballute, beefalo, broccoflower, liger, dramedy, infotainment, Spanglish, zebrass);

- additional relations: 24\% (agitprop, Benelux, kidult, Eurasia, stagflation, tankini, twinight, pro-am);

- polyvalent relations: $11 \%$ (cafetorium, codec, droodle, elevon, modem, ob-gyn, Spork, transceiver, voltammeter);

- tautological relations: 3.5\% (doohickey, hokum, ruckus, wuss);

- other: $3.5 \%$

The relations between blend constituents distinguished by Renner intersect with the ones distinguished in (Wälchli, 2005) for compounds, but it should be kept in mind that all the observations made by Renner can be referred to "coordinative blends" only. Making the criterion of one of the defining ones for "proper" blends would allow to refer to them as to some kind of coordinative complex lexemes alongside with coordinative compounds as opposed to subordinative (determinative) compounds (see (Bauer, 2009) and Scalise and Bisetto (2009) for a detailed classification of compounds).

The criteria of typicality of blends listed by Renner are different from ones selected by, for example, López Rúa. This illustrates that even though the prototypical approach has advantages over the categorical, it can nevertheless be biased by arbitrarily selected criteria, especially if the category in question is as fuzzy and formally diverse as blends. 


\subsection{Gaps and beacons}

Summing up what the literature reveals concerning the structure and formation of blends, the nature of the phenomenon still remain questionable, as well as the outlines of the category itself. Perhaps the best-researched aspect of blends is their structural properties, which go hand-in-hand with general phonological world building laws and which are to a certain extent predictable for any given pair of words that can potentially form a blend. These properties are summarised in Bauer (2012, pp. 14-17) as a set of constraints on form, which are, nevertheless, violated by some existing blends (all examples and counterexamples below are given by Bauer, unless specified otherwise):

1. "In a blend $\alpha \beta$ from words $\alpha \gamma$ and $\delta \beta$, the number of syllables in $\alpha \beta \leq$ the number of syllables in the longer of $\alpha \gamma$ and $\delta \beta "$, e.g. beef( $\varnothing)(b u f f) a l o ~(=\delta \beta)$, ball(oon)(parach)ute $(<\delta \beta)$. But: baro(que)(ro)coco $(=\delta \beta+1)$

2. "In a blend $\alpha \beta$ from words $\alpha \gamma$ and $\delta \beta$, where $\alpha \beta$ is not a monosyllable, $\alpha$ and $\beta$ are each at least one syllable long", e.g. dies(el)(alc)ohol. But: ch(annel)(t)unnel.

3. "The stressed syllable from at least one of the two elements will be retained in the blend. Both may be. There is a preference for the stress pattern of the righthand word to be retained", e.g. posi(tively)(abso)lutely, vodka(Ø)(mar)tini, ox(ford)(Cam)bridge, alpha(betic)(nu)meric. But: ball(oon)(parach)ute.

4. "In a blend $\alpha \beta$ from words $\alpha \gamma$ and $\delta \beta, \gamma$ and $\delta$ are not null elements", e.g. edu(cation)(enter)tainment, tig(er)(li)on. But: key(Ø)(con)tainer ( $\gamma$ is null), jazz(Ø)(ex)ercise, identi(ty)(Ø)kit ( $\delta$ is null).

5. "In a blend $\alpha \beta$ from words $\alpha \gamma$ and $\delta \beta$, where $\alpha$ ends in a consonant and $\beta$ begins in a consonant, the second consonant will be less sonorant than the first", e.g. slan(Ø)(lan)guage. But: West(ern)(Aust)ralia

6. "The blend $\alpha \beta$ must meet all relevant phonotactic requirements", e.g. (from (Kubozono, 1990)) Smoke + drink cannot give /*sməunk/

7. "In a blend $\alpha \beta$ from words $\alpha \gamma$ and $\delta \beta$, where $\alpha \beta$ is a monosyllable, $\alpha$ is a syllable onset and $\beta$ is a syllable rhyme", e.g. $\operatorname{br}$ (eakfast)(I)unch, sm(oke)(f)og, $d($ ove)(h)awk. But: sh(irt)(d)ress (this counterexample is from my collection of blends - NB). 
8. "In a blend $\alpha \gamma \beta$ from words $\alpha \gamma$ and $\gamma \beta$, where $\gamma$ is a phoneme/letter or phoneme/letter string in common between the two base words, this overlap defines the crossover point", e.g. Chicago(go)rilla, gu(ess)estimate. But: h(orse)(z)ebra, tig(er)(li)on, cam(era)(re)corder

9. "In a blend $\alpha \beta$ from the words $\alpha \gamma$ and $\delta \beta$ where $\gamma$ and $\delta$ share no phonemic/orthographic material, the break between $\alpha$ and $\beta$ will fall at a syllable break or, failing that, at an onset/rhyme break", e.g. posi(tively)(abso)lutely. But: tig(er)(li)on.

The fact that one can find counterexamples violating constraints is in itself in accordance with the reality of any living language. To understand the reason why nearly anyone studying blends admits that they are a problematic category, let us consider why it is difficult to distinguish blends from neighbouring morphological categories and why so many examples are either explained and classified controversially by different authors, or treated as marginal cases.

Going back to Marchand, for instance, we can encounter examples given for clipping compounds: while napalm, comintern or positron are mostly treated as 'clipping compounds proper', there are many examples listed by Marchand among clipping compounds that were elsewhere treated as neo-classical compounds (cf. Bauer, 1983) (e.g. lexemes containing mono-, micro-, hydro-, photo-, etc.) or that might be analysed as blends (e.g. greycing $\leftarrow$ greyhound racing; mailomat $\leftarrow$ mail-automat; pulmotor $\leftarrow$ pulmonary motor; Americanadian $\leftarrow$ American + Canadian, etc.). It is necessary to add here that there is a criterion according to which all the coinages combining first part of one word with the second part of another should be called blends (e.g. Kubozono, 1990) and thus positron also falls in the category of blends. The situation is so controversial because sufficient criteria to distinguish between these morphological categories appear not to have been well-elaborated.

Another inconsistency in taxonomy can be traced back to Algeo (1977) who admits that certain forms are treated as blends only as a concession to traditional classifications, but notes that "a consistent taxonomy would regard them merely as contractions" (1977, p. 56). There are a lot of arbitrary classifications, in which categories are outlined according to certain criteria, but even in the framework of one classification it is not always possible to resolve all the cases. Consider, for example, classification by Adams: 
blends are stated to be "made up of two contributory words, one or both of which may be only partially present in the new word" (2001, p. 139), acronyms "may include other than initial letters to make them more word-like: radar" (2001, p. 142). Thus, the exact criteria that would help distinguish between these two categories are not named.

Studying blends in the context of adjacent categories of complex shortenings, López Rúa (2004a, pp. 111-116) criticises traditional approaches and definitions for lack of discriminatory power, lack of homogenity, lack of method for choosing and ranking parameters, lack of category structure, and lack of elaboration. She proposes a prototypical approach to classification, which has advantage over other approaches that it seems to reflect the processes taking place in real language more explicitly. As was stated above, even the advantages of the prototypical approach do not save us from the obvious questions such as what to count as prototype. To agree with Bauer (2012, p. 21), “[w]e need more than new experiments on an ill-defined set of words. We also need a flash of insight, which will allow us to capture the essence of blending and separate it out from everything else (if, indeed, that is the appropriate solution)".

It is not within my capacity to judge whether this research provides such a flash of insight, but its objective is to integrate the previous findings concerning several aspects of blending in one study in order to achieve a new quality of knowledge about this complex phenomenon. The importance of such an approach is spelt out in Gries (2012, p. 165):

More specifically, everything that has been done so far focused on one particular level of resolution: phonemes, graphemes, syllables, and so on. However, this is obviously not how speakers perceive words - naïve speakers have a much more holistic approach, which is why we need measures that allow us to capture and quantify similarity at many different levels at the same time.

This observation was made with regard to similarity, in particular, but its implications, in my view, concern many aspects of blending. Therefore, this research, as outlined in the Introduction, consists of three studies which, on the one hand, use different methods and investigate different sets of data, and on the other hand, are related to each other. The first study (Chapter 4) investigates in what way the phonological properties of the source words influence the form of the shortening and in what way this form is influenced by their semantic relations. The other two studies (Chapters 6 and 7) use the 
findings concerning the phonological, structural and semantic properties of blends to investigate cognitive mechanisms of their formation. 


\section{Chapter 3. Basic terminology and rationale}

This chapter provides definitions to the key terms which are used throughout the thesis and which are crucial for the understanding of the main findings of this research. One might argue that such information could be provided much earlier than 45 pages into the thesis. The previous chapter, however, maps out the route along which the terminology and rationale given below was elaborated, and therefore is an important prerequisite for the following.

\subsection{Approach to defining blends}

As becomes clear from the analysis of the literature in the field presented in the previous chapter, whether a given formation is included in the category of blends or excluded from it depends on the criteria used for defining blends. Currently, there is no unified set of defining criteria for blends, which have often received contradictory definitions in the linguistic literature. Different prototypical features might be chosen depending on whether blending as a word formation type is considered:

1. an instance of compounding (Marchand 1969; Kubozono 1989; Renner 2006);

2. an instance of shortening (Adams 1973; Cannon 1986; Kelly 1998; López Rúa 2002, 2004);

3. a mixture of both processes (Gries 2004, 2006, 2012);

4. a result of an extragrammatical operation of a different nature from both processes (Mattiello, 2013).

If we agree that blends are of the same nature as compounds ( 1 above) then being a twopart formation would indeed become the condition for a blend to be considered prototypical (as stated in Marchand (1969), Kubozono (1990), Kelly (1998), Adams (2001) and other works). Other essential characteristics of blends as an instance of compounding are, e.g., headedness, determinative or coordinative relations between their constituents (see Bauer (2009), Scalise and Bisetto (2009) for detailed classification). The degree of preservation of the original constituents is also important for the comprehension of a blend. Formations combining only the beginnings of the words (in various terminologies - complex clippings / clipping compounds, e.g. digicam $\leftarrow$ digital camera) are difficult to deconstruct into the original constituents without prior knowledge of them, which may be another reason for excluding them from the category of blends (Plag 2003; Gries 2004, 2006). 
On the other hand, if we assume that blending is an instance of shortening ( 2 above), then accepting certain formations as typical members of the category should not depend on the number of their constituents or how the shortening is achieved (i.e. whether the beginning, ending or middle part is preserved). Many researchers, e.g. Algeo (1977), Devereux (1984), López Rúa (2004a), Bauer (2012), include complex shortenings consisting of more than two constituents in the category of blends.

The third approach to defining blends proposed above seems a reasonable alternative, which, however, has to be chosen with regard to the properties of the actual data, not simply to avoid choosing between the first two variants. Therefore, a data-driven approach is taken in this research. The aim will be to describe patterns and regularities which can be observed in a collection of lexical data, and to figure out which of the patterns hold for enough data to be considered category-determining. This approach implies that blending will be considered as subject to general laws of morphological grammar, rather than as an extragrammatical process ( 4 above). Postulating the extragrammaticality of blends (or of clipping compounds, or of any other word formation category) would mean that it is possible, in principle, to make a categorical distinction between grammatical and extragrammatical phenomena. A closer look at real data, however, suggests that this is virtually never the case. On the contrary, even the most 'regular' morphological categories often exhibit fuzzy boundaries and controversial examples (a vast number of which are analysed and discussed in Bauer et al. (2013) on the material of contemporary corpus data ). The data of contemporary morphological studies force the researchers to analyse them in terms of tendencies and probabilities (Bod et al., 2003) rather than in terms of qualitative distinctions. Therefore, the conclusion made in Mattiello (2013, p. 251) that 'the patterns forming blends, acronyms, reduplicatives, and similar formations appear to be best described and explained [...] in terms of prototypical and marginal types' does not necessarily mean that these patterns cannot be woven in the cloth of regular morphology in general.

In the light of the considerations above, a working definition of blends has been developed. The definition is designed primarily to provide grounds for data collection. Therefore, while being consistent with the majority of the previous works published on blends, it does not put any excessive restrictions on what data to include in the scope of the research: 
A blend is a lexical item formed by merging together two (or more) source forms, so that 1) the resulting formation is shorter than its source words put together, either as a result of partial loss of the orthographical and/or phonological material, or as a result of overlap; 2) it has not been formed by concatenation of morphs.

The formal criteria set above do not rule out clipping compounds or three- and fourelement blends, to allow for obtaining a wider spectrum of lexical data. The definition will be reworked at a later stage to account for the results of the actual data analysis. What is important at the moment is to filter out formations which cannot be analysed as either blends or clipping compounds. This includes morphologically complex items formed by concatenation of clippings if the clippings which were established as morphemes before concatenation. For example, the lexeme enviropig is formed by adding a free morph pig to a clipping enviro- (from environment) which is listed in dictionaries (e.g. $O E D$ ) as a morpheme. Therefore, enviropig should be analysed as a compound rather than a blend or a clipping compound. One may argue that listing in a dictionary is not necessarily a sufficient criterion because how morphemes are perceived by language users does not have to be congruent with how dictionaries list them. However, dealing with a fuzzy morphological category implies a need for some boundaries to be imposed to filter out data. Therefore, although I admit that the true state of things in language (e.g. whether a particular clipping has acquired a status of a suffix or prefix) may not be correctly reflected by dictionaries, I choose to rely on dictionaries as orienting beacons which can make the choice of data for the present research better-grounded. A more detailed account of how lexical data was collected for this study with regards to the principles outlined here will be provided in section 4.1. The following section, on the other hand, will be more focussed on what is out of the scope of this research, rather than on what is in it. In what follows I will outline the criteria for data selection and specify the notions important for data analysis.

\subsection{The terminological toolkit and the scope of the study}

In addition to defining what lexemes are the objects of this research (as was done in the previous section), it is important to provide here the approach to selecting lexical material for the study, and the terminology accompanying the key notion of blending, which will be used throughout the following chapters. 
The features nearly all morphologists seem to admit as distinguishing ones for blends is that they are formed out of two constituents which are clipped when being merged together, and that blend formation does not employ morphemes, though even these basic statements do not go without deviations and exceptions. To avoid too many deliberate restrictions on the lexemes that are to be included in the data set for this study, I will include those that are formed of two or more constituents. The maximum number of constituents in the blends exemplified in this thesis is four, this number being used not as a definitive criterion, but only as means of simplifying the data encoding and analysis.

An analysis of the literature on blending has revealed various criteria for determining either whether a formation is a blend at all, or whether a blend is a well-formed, or prototypical, one. In addition to criteria already discussed involving the number of bases taking part in blending, and of whether the bases should be right-clipped or left-clipped, the following criteria for the well-formedness of blends are relevant for data selection:

1. Whether the base elements of a blend should be in co-ordinative semantic relations. The collections of blends in, among others, Kubozono (1990), Kelly (1998), Berg (1998), Renner (2006) and Brdar-Szabó and Brdar (2008) include only lexemes formed out of coordinated bases. Other researchers, such as Algeo (1977), Adams (2001) and Bauer (2012) classify such formations as a subtype of blends. In this research, no restrictions on the semantic origin of blends are imposed, but the semantic type of blends will be accounted for in the data analysis.

2. Whether base elements of a blend are reversible. This criterion was suggested in Algeo (1977) as a tendency, rather than a definitive constraint. It seems that blends which conform to the reversability criterion are relatively rare, and moreover, for many blends the order of the components is determined by either semantic or phonological factors. Semantically, reversibility of constituents is, in principle, possible only if they are in coordinative relations, while the scope of this study is not restricted to such relationships, as stated above. But even in the case where there are no semantic restrictions, the order of constituents can be subject to such factors as frequency, length, and prosody. Therefore, the reversibility of components will not be used as a restriction on data selection. 
3. Whether at least one of the words that are blended necessarily undergoes truncation. For example, according to López Rúa (2004b, p. 64) only those blends which demonstrate a 'medium degree of shortening' deserve to be named prototypical. A restriction like this would mean excluding words formed by two overlapping words e.g. stoption $\leftarrow$ stop + option, which, apart from being very interesting material to study, are not infrequent. Hence, in this research these are included but their structural type is kept in mind (see the classification of blends into structural types in section 4.1)

The lexical units that come into blends are referred to as source words (Cannon, 1987; Gries, 2004a; Kemmer, 2003; Kubozono, 1990; Lehrer, 1998), source forms (López Rúa, 2002, 2004a, 2004b, 2007), component words (Kelly, 1998), input words (Brdar-Szabó and Brdar, 2008) or formatives (Fradin, 2000). The most widely accepted term 'source words' will be used throughout this thesis. For cases when a different unit, for example, an affix (see below) becomes a blend constituent, these units will be labelled as 'source forms', but cases like these are marginal.

The disagreement concerning the parts of source words that actually become blend constituents exists both in terms of labelling them and in terms of counting them as blend constituents with regard to their (non)morphemic nature. The most widely used term for blend parts is 'splinters' (Adams, 1973; Bertinetto, 2001; Fandrych, 2008a; Jin, 2005; López Rúa, 2004b). However, this term is used in at least two different senses. According to one interpretation, any 'shorter substitutes' of words (Adams, 1973, p. 142) should be called splinters. The other approach is to use this term only for those word parts that have started to be used productively in more than one blend, e.g. -(a)holic, -(a)nomics (Bauer, 2006, p. 503). This labelling, however, is potentially the cause of unnecessary polysemy of the term. If splinters are only the forms that have already demonstrated some productivity, then it is not clear how to distinguish between splinters and bound morphs. Indeed, some researchers use the terms bound morphemes (Lehrer, 1998), combining forms (Lehrer, 1998; B. Warren, 1990) or bound splinters (Fandrych, 2008a) for blend constituents that are used with some regularity. Moreover, some of the morphs that are referred to as splinters in publications on blends (e.g. -ware, $e_{-,},-$holic), are listed as morphemes in contemporary dictionaries.

In this research I will use the term 'splinters' for any word parts that come into blends, with two necessary conditions: 1 ) they are not full words; 2 ) they are not morphemes or 
bound morphs registered in dictionaries (OED and $C E D$ were used to check this). The second condition requires an additional clarification. In some cases a bound morph, e.g. a neo-classical combining form, is merged together with a truncated form of another word, i.e. with another splinter, as in pro-mia $\leftarrow$ pro + bulimia. In this case the resultant lexical item will be counted as blend originated from a combining form and a word. There are also cases in which the part of the source word that is preserved in the blend is a morpheme (if one of the source forms is a compound or another morphologically complex word), i.e. cookprint $\leftarrow$ cook + footprint. The resulting form is considered a blend because it was formed not by compounding or derivation, i.e. putting two words together or adding affixes to a base, but by merging together already complex lexical units with deleting part of their orthographical and/or phonological material.

It is not possible in all cases to state that blends consist of splinters. In overlapping blends like stoption the source words, technically, are preserved in full, but part of their phonological / orthographical material is actually lost, otherwise there would have been some repetition. Because of the overlap, it is impossible to say which of the source forms loses its part. As a result, not all material of both source words is preserved, and thus the new lexical unit is a blend, but each of the source forms separately is preserved in full, and thus there are no splinters.

The scope of the research is restricted to blends, and not to neoclassical compounds or regular compounds. This explains why it is important to restrict the lexical data to be analysed to formations made up of full words or splinters, and not of free morphs, or neoclassical combining forms. Section 4.1 provides a detailed account of how this is controlled. The only thing which remains to note here is that the lexical material of this study will be restricted to relatively recent blends. This is done for practical reasons: the source words of newly coined blends can be traced relatively easily, which, in its turn, makes it easier to control and analyse the parameters of blends and their source words which have been specified. 


\section{Chapter 4. Lexical data: From structure to meaning and back again1}

This chapter aims to analyse a corpus of contemporary English blends (including formations which may be analysed as clipping compounds) with respect to their phonological, structural and semantic properties, in order to find evidence that would help clarify their status in the system of English word formation. The methods of data sampling and the classification criteria are covered in section 4.1. Sections 4.2 and 4.3 focus on the statistical analysis of the formal and phonological properties of the words in the collected corpus, adding to the previous studies in this field, in particular those concerned with the distinction between blends and clipping compounds. Section 4.4 goes one step further in explaining the phonological and structural properties of different types of formations, taking into consideration their semantics and origin.

\subsection{Data sampling and methodology}

The lexical data were obtained from a number of online collections of neologisms and occasionalisms: Word Spy http://wordspy.com/ Urban Dictionary http://www.urbandictionary.com/ The Rice University Neologisms Database created by Suzanne Kemmer (http://neologisms.rice.edu/index.php), The Global Language Monitor http://www.languagemonitor.com, Macmillan Dictionary online http://www.macmillandictionary.com, the Unword Dictionary http://www.unwords.com, The Word of the Year collections on http://www.merriamwebster.com/info/woy archive.htm, as well as from opportunistic sources such as newspapers and magazines. The collection included all blends which appeared in the sources no earlier than January 1, 2000 (a randomly chosen date, but one which allows for a sufficient number of novel formations to be collected). If the source of the blend words provided no date of the first occurrence (which was frequently the case), the blend was looked up in the Corpus of Contemporary American English (COCA), and a Google search within a timeframe of January 1, 1990 to January 1, 2000 was performed. A blend was excluded from the data set if: 1) it was dated in COCA before January 1 , 2000 (e.g. boomburb), or 2) it was found in Google with occurrences before January 1, 2000 (e.g. peoplerazzi).

The choice of words for the data set was subject to the working definition of blends provided in section 3.1. In particular, their formation had to involve partial loss of the material of the source words, and had not to be analysable as concatenation of morphs.

\footnotetext{
${ }^{1}$ This chapter is a revised version of Beliaeva (2014)
} 
The second condition was accomplished by looking up the blend parts in the Oxford English Dictionary Online (OED). If the $O E D$ listed a blend part as a morpheme, e.g. $e$-, -tastic, the words that were formed by adding such a morpheme to a full stem, e.g. e-cigarette, killtastic, were excluded from the data set (apart from formations like e-linquent, which are not exhaustively analysable into morphs). The same applies to established clipped forms, e.g. frat for fraternity, jack for hijack because the words that contain them can be analysed as compounds rather than blends, e.g. nerdjack $\leftarrow$ nerd + jack (see also examples in Table 1).

Table 1. Derivatives and compounds formed by adding recently established morphs

\begin{tabular}{|c|c|c|c|}
\hline Morph & $\begin{array}{l}\text { Type of morpheme } \\
\text { (according to OED) }\end{array}$ & Meaning & Example \\
\hline$e-$ & prefix & $\begin{array}{l}\text { denoting the use of } \\
\text { electronic data } \\
\text { transfer }\end{array}$ & $e$-cycling \\
\hline$m-$ & prefix & $\begin{array}{l}\text { denoting commercial } \\
\text { activity conducted } \\
\text { electronically by } \\
\text { means of mobile } \\
\text { phones }\end{array}$ & m-commerce \\
\hline -(a)delic & suffix & $\begin{array}{l}\text { forming adjectives } \\
\text { denoting musical } \\
\text { genres or styles that } \\
\text { incorporate } \\
\text { psychedelic music } \\
\text { with another element }\end{array}$ & Celtadelic \\
\hline -logue & combining form & denoting compilation & civilogue \\
\hline -licious & combining form & $\begin{array}{l}\text { denoting someone or } \\
\text { something delightful } \\
\text { or extremely } \\
\text { attractive }\end{array}$ & beaulicious \\
\hline -tastic & combining form & $\begin{array}{l}\text { denoting someone or } \\
\text { something regarded } \\
\text { as an extremely good } \\
\text { example of their } \\
\text { particular type }\end{array}$ & killtastic \\
\hline enviro & clipping & $\begin{array}{l}\text { short for } \\
\text { environmentalist, } \\
\text { environmental }\end{array}$ & enviropig \\
\hline jack & clipping & short for hijack & nerdjack \\
\hline
\end{tabular}


Defining the number of source forms of a particular blend sometimes required a wider context than just the morphological constituents. Thus, on-call-ogist can be analysed as a three-constituent blend, i.e. on + call + oncologist, but it is obvious from the context in which it appears, shown in (4.1), that it is a blend of on call and oncologist, and, therefore has two source forms, as the majority of the blends do. On the other hand, Thankshallowistmas $\leftarrow$ Thanksgiving + Halloween + Christmas is definitely a three-part blend.

(4.1) She looked at me, her eyebrows twisted. "Yeah, I'm on call three times a week." "Doesn't that mean you're an on-call-ogist?" (McFedries 2011)

Using these methods, 506 neologisms were collected, among which 415 nouns, 50 adjectives, 39 verbs and 2 adverbs (the full list is given in Appendix 1). Out of those, 15 words can be analysed as either nouns or verbs (e.g. dweet / to dweet $\leftarrow$ drunk + tweet / $d r i n k+$ tweet) and 6 as either nouns or adjectives (e.g. cinematard $\leftarrow$ cinema + retard). In each case a category ambiguous word was assigned to one of the categories according to the use in the sources the word was found in, or, if usage in both categories was attested, to the category which corresponded to the majority of the examples that could be found. The vast majority of the data are two-constituent formations, 8 are three-constituent, and 4 are four-constituent.

There are parts of blends, which, although not listed in the $O E D$ as combining forms, clipped forms or other kinds of morphemes, seem to demonstrate a certain productivity, as they appear in more than one blend either in the present collection or in COCA. For example, two blends in our data have the final part -coustic: elecoustic, funkcoustic; the COCA search provides 10 words ending in -zilla: bridezilla, groomzilla, etc. The forms like -coustic or -zilla are what Lehrer (1996, p. 361) calls 'independent bound morphemes', or what in other sources (e.g. Bauer 2006; Bauer et al. 2013) are referred to as splinters which are used productively in more than one word. Overall, 25 initial and 52 final splinters in the data set can be regarded as productive to a greater or lesser extent. They appear in 150 blends in total, which comprises a little over $28 \%$ of the data. Excluding such words from the corpus of blends would mean taking a somewhat extreme approach and stating that the only formations that can be classified as blends are those that merge their source words in a unique way so that the same splinter has never been used to form other words. A classification of this kind would risk the establishment of a maze of categories even more ill-defined than the one I aim to pin 
down. Moreover, in the course of data analysis, whatever method was used (see below), the fact that a splinter could be found in one blend or in more than one did not appear to be a factor that influenced the form of the blend. Therefore, blends with productive splinters remain in the corpus. Their presence shows that, given appropriate conditions, a part of a blend may undergo a gradual process of turning into a productive splinter and eventually into a morpheme. The conditions that determine the productivity are outside the scope of this study, but the fact that this process takes place is another reason to focus on relatively novel blends.

To get a better understanding of how exactly the source forms are merged into blends, their phonological, structural, and semantic properties were considered. The phonemic transcriptions of the source words for all the blends were acquired from Cambridge English Pronouncing Dictionary (CEPD). For obvious reasons, the transcriptions of the blends themselves could not be found in any dictionaries, and not all the sources gave transcriptions. If the source provided a transcription of the blend, it was brought into compliance with the notation of $C E P D$. If no transcription of the blend was provided, it was compiled from the transcriptions of the source words, in which case the country of the blend's origin was born in mind and the corresponding variant of transcription was chosen, e.g. the US English transcription /kwa:n.zə/ for Kwanzaa and /'ræm.ə.dan/ for Ramadan in a Northern American origin blend Christmahanukkwanzadan. In the presentation of American transcriptions, the superscript for sounded r was omitted for simplicity reasons, e.g. /ə/ instead of / $/ \partial^{r}$ / is recorded. Quality changes of the sounds were taken into consideration when deciding which phonemes were preserved in the blends and which not. For example, even though on the graphical level all but one of the letters of the word mascara are preserved in the blend mascary $\leftarrow$ mascara + scary, on the phonological level only /mæs'k/ of /mæs'ka:.rə/ is preserved in /mæ'skeə.ri/, because the stressed vowel comes from the second source word scary. Therefore, the lengths of splinters and the degree of overlap for some of the blends are different depending on whether graphemes or phonemes are the basis of the analysis.

For the purposes of computational analysis all transcriptions were re-coded using the adapted version of the Speech Assessment Methods Phonetic Alphabet (SAMPA) (Wells 1997) based on IPA. The statistical analysis of the data was performed using R software package (R Development Core Team 2012). For various steps of data analysis different 
statistical methods were used, and a description of each particular method will follow below where appropriate.

It is widely accepted (e.g. Cannon 1986; Renner 2006; Brdar-Szabó and Brdar 2008) that the majority of blends combine the initial part of the first source word (W1) with the final part of the second source word (W2); in terms of the formula from Plag (2003, p. 123):

(4.2) $\quad \mathrm{AB}+\mathrm{CD}=\mathrm{AD}$

The formula in (4.2) does not provide sufficient elements to deal with all the data collected, particularly when more than two source words were involved. As a result of applying the logic of Plag's formula to the lexical data, depending on how many source words took part in the formation and what parts of the source words were preserved, new labels for structural types were created, as shown in (4.3). The parts of the source words that do not enter blends are put in parentheses.

a) $\mathrm{AB}+\mathrm{CD}=\mathrm{AC}$ : for initial-initial splinter formations, e.g. hydrail $\leftarrow$ hydr(ogen) + rail(way)

b) $\mathrm{AB}+\mathrm{CD}+\mathrm{EF}(+\mathrm{GH})=(\mathrm{X})$ : for three- or four-constituent blends, e.g. SoLoMo $\leftarrow$ $s o($ cial $)+l o($ cal $)+m o($ bile $)($ ACE $)$, bastitcherbator $\leftarrow$ bast $($ ard $)+($ b)itch + (mast) urbator (ADF), afflufemza $\leftarrow$ afflu(ent) + fem(inist) + m(others) + (influen)za $(\mathrm{ACEH})$

The labelling system had also to account for cases of full preservation of one or more of the source words, as in alcoholimia $\leftarrow$ alcohol $+($ bu)limia. As a result, all the collected neologisms were classified into structural types as illustrated in Table 2: 
Table 2. Structural classification of lexemes in the collected corpus

\begin{tabular}{|c|c|c|c|}
\hline Blend type & Structure & $\begin{array}{l}\text { Number of } \\
\text { tokens }\end{array}$ & Examples \\
\hline WD & $\begin{array}{l}\text { all of } W 1+\text { the end of } \\
W 2\end{array}$ & $172(34.1 \%)$ & $\begin{array}{l}\text { alcoholimia } \leftarrow \text { alcohol }+ \\
\text { (bu)limia }\end{array}$ \\
\hline $\mathrm{AD}$ & $\begin{array}{l}\text { the beginning of } \mathrm{W} 1+ \\
\text { the end of } \mathrm{W} 2\end{array}$ & $157(31.0 \%)$ & $\begin{array}{l}\text { blizzaster } \leftarrow \text { blizza }(r d)+ \\
\text { (dis)aster }\end{array}$ \\
\hline AW & $\begin{array}{l}\text { the beginning of } \mathrm{W} 1+\text { all } \\
\text { of } \mathrm{W} 2\end{array}$ & $82(16.2 \%)$ & fabulash $\leftarrow$ fabul(ous) + lash \\
\hline WW & W1 + W2, overlap & $29(5.7 \%)$ & flabdomen $\leftarrow$ flab + abdomen \\
\hline $\begin{array}{l}\text { central } \\
\text { replacement }\end{array}$ & $\begin{array}{l}\text { W2 is inserted in the } \\
\text { middle of W1 (W1 and } \\
\text { W2 labels could be used } \\
\text { the other way round, the } \\
\text { choice of W1 is based on } \\
\text { what word provides the } \\
\text { beginning for the blend) }\end{array}$ & $20(3.9 \%)$ & $\begin{array}{l}\text { parahawking } \leftarrow \text { para(glid)ing } \\
+ \text { hawk }\end{array}$ \\
\hline $\mathrm{AC}$ & $\begin{array}{l}\text { the beginning of } \mathrm{W} 1+ \\
\text { the beginning of } \mathrm{W} 2\end{array}$ & $19(3.7 \%)$ & $\begin{array}{l}\text { hydrail } \leftarrow \text { hydr(ogen })+ \\
\text { rail }(\text { way })\end{array}$ \\
\hline $\mathrm{ACF}$ & $\begin{array}{l}\text { the beginning of } \mathrm{W} 1+ \\
\text { the beginning of } \mathrm{W} 2+ \\
\text { the end of } \mathrm{W} 3\end{array}$ & $2(0.4 \%)$ & $\begin{array}{l}\text { Thankshallowistmas } \leftarrow \\
\text { Thanks(giving) }+ \text { Hallow(een) } \\
+(\text { Chr)istmas }\end{array}$ \\
\hline WC & $\begin{array}{l}\text { all of } W 1+\text { the } \\
\text { beginning of } W 2\end{array}$ & $4(0.8 \%)$ & $\begin{array}{l}\text { Obamacon } \leftarrow \text { Obama }+ \\
\text { con(servative) }\end{array}$ \\
\hline $\mathrm{BD}$ & $\begin{array}{l}\text { the end of } \mathrm{W} 1+\text { the end } \\
\text { of } \mathrm{W} 2\end{array}$ & $2(0.4 \%)$ & frohawk $\leftarrow(a)$ fro $+(M)$ ohawk \\
\hline ACE & $\begin{array}{l}\text { the beginning of } \mathrm{W} 1+ \\
\text { the beginning of } \mathrm{W} 2+ \\
\text { the beginning of } \mathrm{W} 3\end{array}$ & $4(0.8 \%)$ & $\begin{array}{l}\text { SoLoMo } \leftarrow \text { so(cial) }+\mathrm{lo}(\mathrm{cal})+ \\
\text { mo(bile })\end{array}$ \\
\hline ACW & $\begin{array}{l}\text { the beginning of } \mathrm{W} 1+ \\
\text { the beginning of } \mathrm{W} 2+\text { all } \\
\text { of } \mathrm{W} 3\end{array}$ & $1(0.2 \%)$ & $\begin{array}{l}\text { Chindonesia } \leftarrow \text { Chin }(a)+ \\
\text { Ind }(\text { ia })+\text { Indonesia }\end{array}$ \\
\hline ACEH & $\begin{array}{l}\text { the beginning of } \mathrm{W} 1+ \\
\text { the beginning of } \mathrm{W} 2+ \\
\text { the beginning of } \mathrm{W} 3+ \\
\text { the end of } \mathrm{W} 4\end{array}$ & $1(0.2 \%)$ & $\begin{array}{l}\text { afflufemza } \leftarrow \text { afflu(ent) }+ \\
\text { fem(inist) }+m \text { (others) }+ \\
\text { (influen)za }\end{array}$ \\
\hline
\end{tabular}




\begin{tabular}{|c|c|c|c|}
\hline Blend type & Structure & $\begin{array}{l}\text { Number of } \\
\text { tokens }\end{array}$ & Examples \\
\hline ACWH & $\begin{array}{l}\text { the beginning of } \mathrm{W} 1+ \\
\text { the beginning of } \mathrm{W} 2+\text { all } \\
\text { of } \mathrm{W} 3+\text { the end of } \mathrm{W} 4\end{array}$ & $1(0.2 \%)$ & $\begin{array}{l}\text { Christmahanukkwanzadan } \leftarrow \\
\text { Christma }(\text { s })+\operatorname{Hanukk}(a)+ \\
\text { Kwanzaa }+(\text { Rama }) \text { dan }\end{array}$ \\
\hline $\mathrm{ADF}$ & $\begin{array}{l}\text { the beginning of } \mathrm{W} 1+ \\
\text { the end of } \mathrm{W} 2+\text { the end } \\
\text { of } \mathrm{W} 3\end{array}$ & $1(0.2 \%)$ & $\begin{array}{l}\text { bastitcherbator } \leftarrow \text { bast }(\text { ard })+ \\
\text { (b)itch }+(\text { mastu }) \text { rbator }\end{array}$ \\
\hline acronymic & $\begin{array}{l}\text { initial letters of two or } \\
\text { more source words } \\
\text { combined with another } \\
\text { word or part of it }\end{array}$ & $11(2.2 \%)$ & $V B 6 \leftarrow v($ egan $) b$ (efore) six \\
\hline total & & $506(100 \%)$ & \\
\hline
\end{tabular}

The distinction between, for example, AD and WD blend types is a result of a common practice which is to distinguish between blends consisting of shortened versions of their source words and those preserving one or both of them in full, made, for example, in Algeo (1977) and Gries (2004). This distinction, however, is less clear-cut than it may seem. For example, if the structural types of weisure $\leftarrow$ work + leisure /'wez.ə $\leftarrow \mathrm{w}(3: \mathrm{k})+$ ('l)ez.ə/and dramality $\leftarrow$ drama + reality /dra'mæl.ı.ti $\leftarrow$ 'dra:.m(ə) + (ri).'æl.ı.ti/ are defined relying on their transcriptions, they both are AD blends, but the first source word of weisure loses most of its phonological material when it enters the blend, while /'dra:.mə/ in dramality is almost fully preserved, apart from the last schwa vowel which is replaced by the stressed /æ/ from /ri.'æl...ti/. Moreover, drama is fully retained graphically, and dramality could be labelled as WD, relying on orthography rather than phonology. Therefore, what seems rational for the structural analysis in this study is to rely on a quantitative characteristic (i.e. the number of phonemes preserved in a splinter) instead of a qualitative one (i.e. full or partial preservation of the source word). The degree of preservation of W1 or W2 can then be calculated as percentage of the W1 or W2 phonemes (or graphemes, in relevant analyses) preserved in a splinter. This means there is no need to label some of the blends as WD, as AD can be sufficient for all blends preserving the beginning or all of the first source word (henceforth W1). The same applies to formations preserving either the beginning or all of W1 plus the beginning of W2 (this shortening will henceforth stand for the second source word), which will be labelled as AC (rather than distinguishing between $\mathrm{AC}$ and WC). 
It is clear, though, that the same logical operation does not apply to words like hiberdating $\leftarrow$ hiber(nate) + dating, which can be referred to as either AC or AD forms, depending on whether to count the letters/phonemes in dating from left to right (assuming it is an 'extended beginning' of W2) or from right to left (for 'extended ending'). For these formations the label AW will be used, to avoid potential ambiguity. The same principles apply to labelling overlapping blends like stoption $\leftarrow$ stop + option, preserving both the source words in full. They will be referred to as WW blends.

Table 3. Structural classification of lexemes in the collected corpus, revised

\section{Blend type Structure}

\section{Number of Examples \\ tokens}

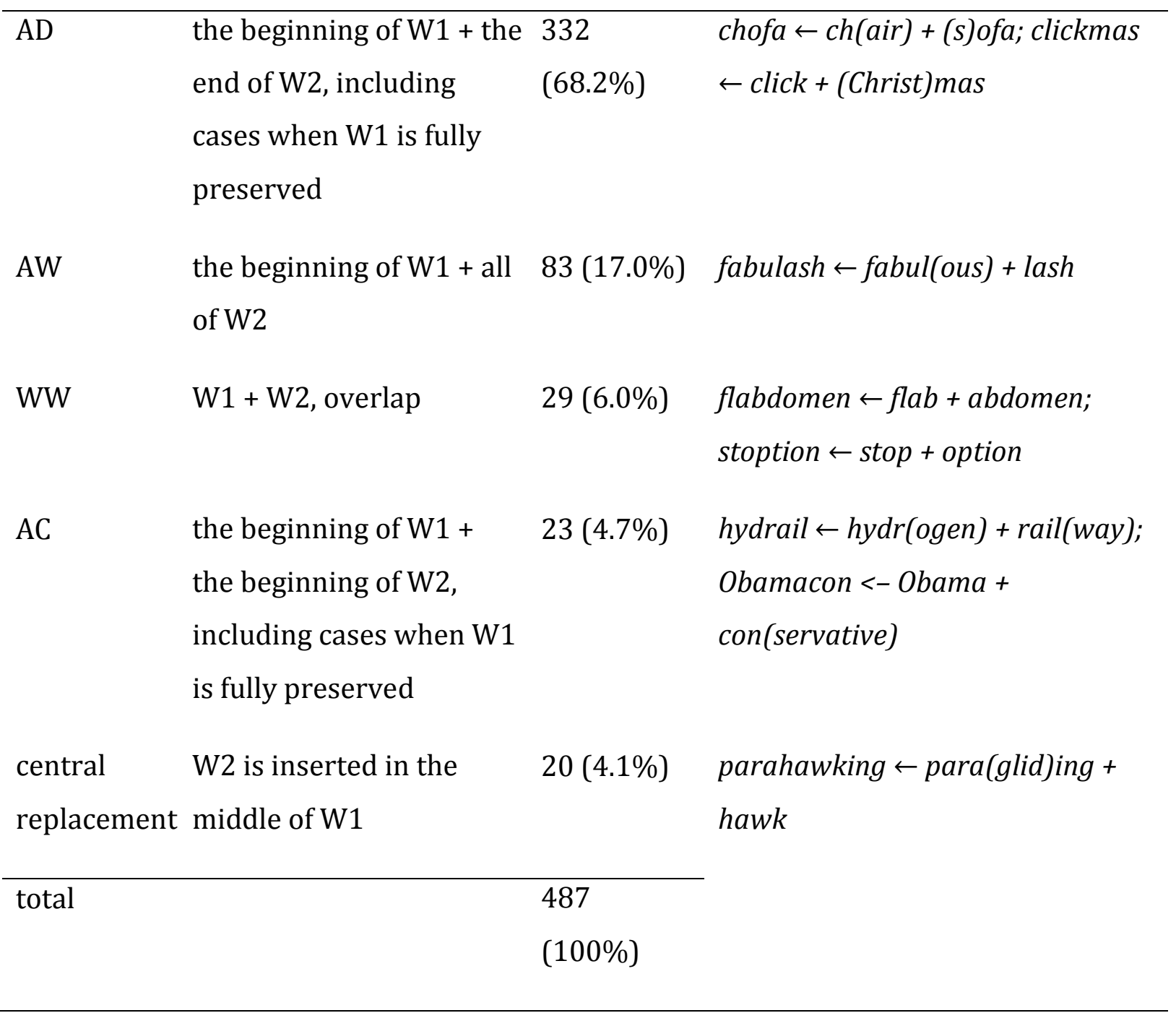

Thus, after the most marginal types (represented by fewer than 10 tokens) were excluded from the analysis, the majority of the blends in our corpus was classified into 5 categories represented in Table 3. 
The only thing that remains to be mentioned regarding the structural types of blends is that 59 blends $(11.6 \%$ of the total corpus, $11.9 \%$ of the corpus excluding the marginal types) have a non-central overlap, i.e. their source words have one or more coinciding letters/phonemes either at the beginning (e.g. protoduct $\leftarrow$ prototype + product) or at the end (e.g. hiberdate $\leftarrow$ hibernate + date). These blends can be either treated as a separate structural type, or be subject to the general structural classification, as shown in Table 4.

Table 4. Structural types of blends with non-central overlap

\begin{tabular}{lll}
\hline $\begin{array}{c}\text { Blend } \\
\text { type }\end{array}$ & Number of tokens & \multicolumn{1}{c}{ Examples } \\
\hline AD & $31(52.5 \%)$ & protoduct $\leftarrow$ proto(type $)+$ product \\
WD & $17(28.8 \%)$ & parentnoia $\leftarrow$ parent + par $($ a $)$ noia \\
AW & $10(16.9 \%)$ & hiberdate $\leftarrow$ hiber $(n)$ ate $+(d)$ ate \\
BD & $1(1.7 \%)$ & Podestrian $\leftarrow(i) P o d+\boldsymbol{p}(e)$ destrian \\
\hline total & $59(100 \%)$ & \\
\hline
\end{tabular}

All statistical analyses that are reported below have been run both excluding these blends and including them. The overall results do not change significantly whether the corresponding groups of blends include these items or not. Therefore, for the sake of consistency of the analysis, all the blends with non-central overlap were classified into the structural types shown in Table 3 applying the approach described above.

\subsection{Phonological properties}

\subsubsection{Data and methods}

Previous findings concerning the phonology of blends discussed in Chapter 2 have shown that blends are subject to prosodic rules, and that the elements that are used as building blocks for blends are syllabic substructures, i.e. onsets, rimes and codas (Kubozono 1990; Kelly 1998; Bauer 2012), rather than individual phonemes. However, the fact that syllabic constituents play a role in the structure of blends does not mean that individual phonemes do not matter. The following analysis takes a bottom-up strategy and is undertaken in order to detect any properties of the individual phonemes which influence the probability of their preservation in the splinters. To estimate this, the relationship between individual phonemes and the length of splinters in different types of blends is investigated. 
One of the main findings in Gries (2006) is that the switch point in clipping compounds is placed earlier than in blends. The same differences can be observed in terms of the degree of source word preservation in $\mathrm{AC}$ and $\mathrm{AD}$ forms in our corpus of neologisms. The relative proportions of source words preserved in AC and AD formations are shown in Figure 2. The difference in preservation of W1 can also be observed between AC and AW (for obvious reasons, it is impractical to compare the preservation of W2 in AW with $\mathrm{AC}$ or $\mathrm{AD}$, and also to include $\mathrm{WW}$ forms in this comparison).
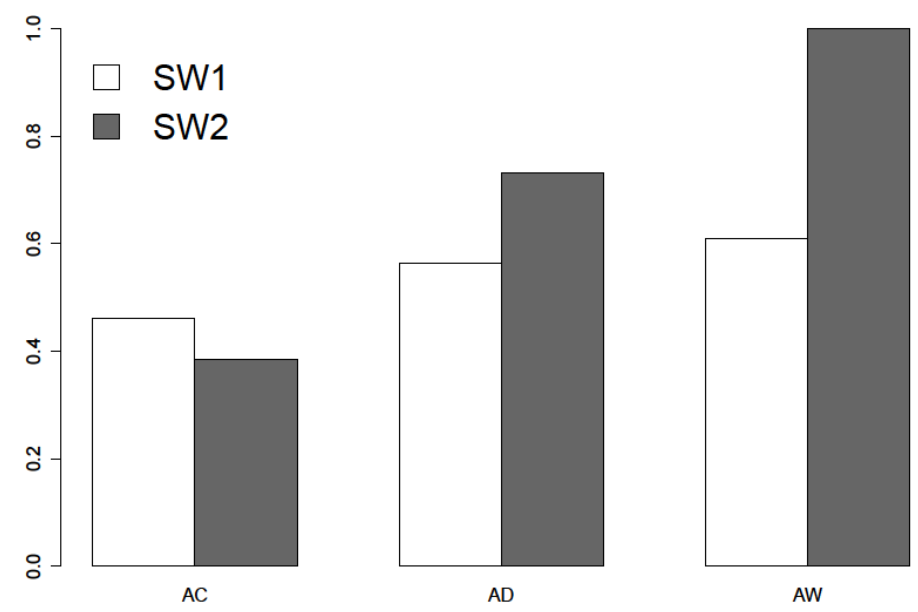

Figure 2. The proportion of the first (SW1) and the second (SW2) source words preserved in formations of different structural types

The coiner of a blend has to decide (although such a decision is not claimed to be a conscious one) where to place the switch point. One of the factors which influences this decision is how large a portion of the beginning or the end of the source word needs to be preserved and, respectively, how many phonemes of each source word the splinters will include. An outcome that reflects this decision is splinter length: the more phonemes are included, the longer the splinter, and vice versa. If individual phonemes can influence the position of the switch point, this can be revealed in the form of a correlation between the splinter length and some relevant characteristics of the phonemes.

An analysis including all the phonemes preserved in blends and clipping compounds would have to account for various characteristics such as the distance from the phoneme and the beginning / end of the word, the mutual alignment of the phonemes, etc. Such an analysis, however promising it might seem, lies outside the scope of the present study, therefore, a simpler analysis will be carried out, focussing only on the 
phonemes situated in close proximity to the switch point. In particular, I will look at the relationship between splinter length and 1) the phonemes placed next to the switch point in each splinter (from here onwards they will be labelled as 'boundary phonemes'), and 2) the phonemes one place to the left or to the right of them (depending on whether the splinter was initial or final). In the initial, i.e. left-hand, splinter of the blend the boundary phoneme was coded as L1 (W1L1 in the first source word and W2L1 in the second source word), and the phoneme to the left of it as L2 (W1L2 or W2L2, respectively). The boundary phoneme in the right-hand splinter was coded as R1, and the phoneme to the right of it as R2, which is shown in Figure 3.

AD: a) with overlap: blizzaster

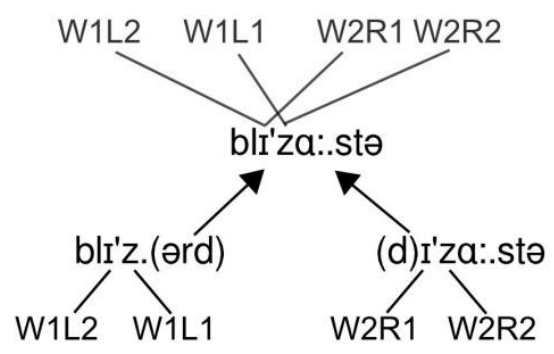

b) without overlap: spime

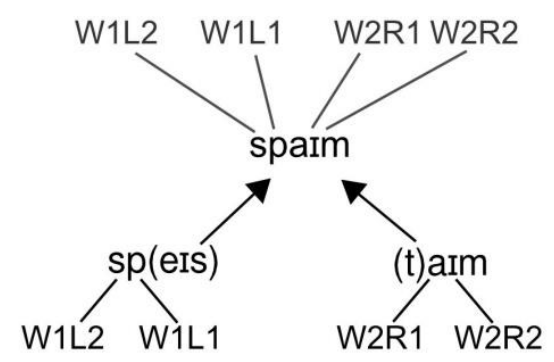

AC:

scigov

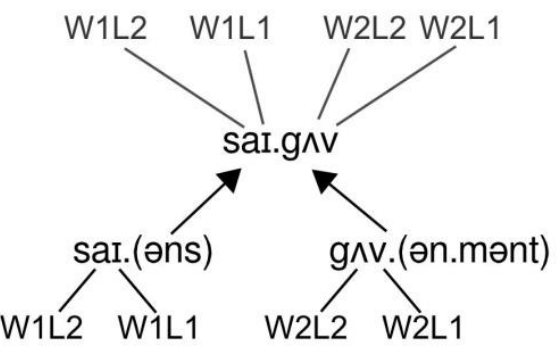

Figure 3. Boundary phonemes in blends and clipping compounds.

Black lines indicate the position of boundary phonemes in the source words, grey lines indicate their position in the resulting formation.

In each case, boundary phonemes were coded with respect to their position in the splinter of a source word. Therefore, in overlapping blends some phonemes may be coded twice, e.g. in guyliner /'gai,laı.nə/ $\leftarrow$ guy /gaı/ + eyeliner /'aı,laı.nə/, /aI/ is coded both as W1L1 and as W2R1. In the further analysis, the effects of double coding were taken into consideration. As the previous example shows, if full word becomes part of a blend, it is the initial or final phonemes (depending on the position of the word in the resulting blend) that are coded as boundary phonemes. 
The purpose of the analysis is to figure out whether there is any association between the length of the splinter and the ranking of any of the two phonemes in close proximity to the switch point (accounting also for the fact that longer splinters may come from longer source words). The analysis included pairwise correlations between W1 and W2 splinter length and: 1) whole blend length, 2) source word length, 3) sonority/frequency ranks of boundary phonemes.

The sonority ranking was adapted from Giegerich (1992, p. 152), grading from the most sonorant low vowels to voiceless stops with minimal sonority. As for frequency ranking, two methods were used. Firstly, the relative token frequencies of phonemes were adapted from Mines et al. (1978) who used a database containing 103,887 phoneme occurrences taken from casual conversational American English obtained from recorded interviews. In addition to that, the frequency with which each phoneme appeared in the current data set was calculated. All the frequency-based analyses (see below) showed similar results with both measures of frequency.

It is important to note that the labels for the structural types (AC, $\mathrm{AD}$ and others) will not be used as variables in any of the simple correlation analyses. The terms 'initial /final splinter' and 'left /right splinter' mentioned interchangeably in section 4.2.2 reflect the position of the splinter in the source word, not in the resultant formation. Therefore, the terms 'initial splinter' or 'left splinter' refer to the A part of AC, AD, and $\mathrm{AW}$ forms, as well as the $\mathrm{C}$ part of the $\mathrm{AC}$ forms. Likewise, the terms 'final splinter' or 'right splinter' refer to the $\mathrm{D}$ part of $\mathrm{AD}$ forms, the $\mathrm{W}$ part of $\mathrm{AW}$ forms, and the right part of the WW forms.

\subsubsection{Results and discussion}

The correlation analysis shows that both the initial and the final splinter lengths are related to:

1) the length of the whole blend,

2) the source word lengths, and

3) the sonority and frequency ranks of the boundary phonemes.

Both W1 left splinter length and W2 right splinter length are positively correlated with the length of the whole blend $(r=0.64, p<0.001$ for the correlation between blend length and the length of the left splinter, $r=0.48, p<0.001$ for the correlation between blend 
length and the length of the right splinter). This includes cases when the W1 left splinter equals W1, as in parentnoia, or when the W2 right splinter equals W2, as in fabulash.

Accordingly, W1 left splinter length is positively correlated with the length of W1 $(\mathrm{r}=0.53)$, and W2 right splinter length with the length of W2 ( $\mathrm{r}=0.79)$, and both correlations are statistically significant ( $\mathrm{p}<0.001$ for each correlation, see Figure 4 ). This means that in case of blending the beginning or the whole of W1 with the ending or the whole of W2, longer source words result in longer splinters, i.e. the tendency is to preserve an amount of phonological and graphical material that is proportional to the length of the source word.
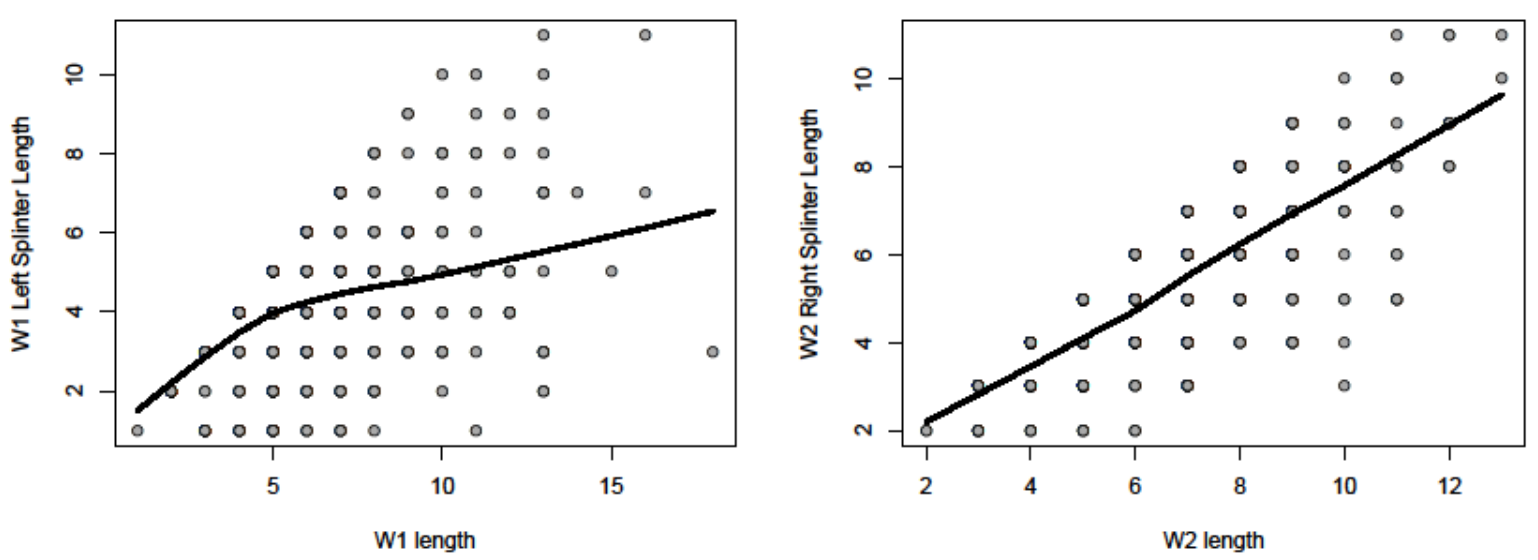

Figure 4. Correlation between the length of the splinters and the length of the source words. Left plot: the correlation between the number of phonemes in the left / initial splinter and WI length in phonemes. Right plot: the correlation between the number of phonemes in the right / final splinter and W2 length in phonemes. Darker circles represent multiple data points. Each relationship is graphically expressed by a lowess line.

Note that the correlation coefficient is higher for W2, that is, the relationship between the length of the final splinter and the length of W2 is stronger than between the length of the initial splinter and the length of W1. However, if the initial splinter of W2 is preserved (i.e. in clipping compounds) no significant correlation is found between the length of the splinter and W2 length ( $\mathrm{r}=0.22, \mathrm{p}=0.0872)$. This means that the observed correlations between the lengths of the splinters and the lengths of the source words the splinters originate from does not simply reflect the fact that longer words produce longer splinters. If this were true, the correlations between splinter lengths and the source word lengths would be similar for W1 and W2. On the contrary, the correlation between the initial splinter length and the length of the source word is observed for W1, but not for W2. Initial splinter of W2 is found only in clipping compounds. Therefore, the 
observed difference indicates that the splinters of AC-forms are shortened with less regard to how much of the source word is retained than the splinters of other forms in the present collection. Such a conclusion, however, has to be treated with caution because the absence of the correlation between W2 initial splinter length and the length of W2 may be due to low number of observations (only $23 \mathrm{AC}$ formations in the collected data set).

The relations between the splinter length and the ranking of the boundary phonemes depend on whether the splinter is initial (left) or final (right). The correlation between the length of the left splinter and the sonority of the boundary phonemes turned out to be close to zero ( $r s=0, p=0.928$ for the correlation between the length of the left splinter and W1L1 sonority, $r s=-0.08, p=0.0894$ for the correlation between the length of the left splinter and W1L2 sonority, rs stands for Spearman's correlation coefficient, which is used here and below when dealing with ranked data). This means that the sonority of the phonemes in the data has no effect on the probability of them being included in the initial splinter. There is, however, a weak correlation between the sonority rank of the boundary phonemes in the right splinter and the right splinter length ( $\mathrm{rs}=0.1, \mathrm{p}=0.0345$ for the correlation between the length of the right splinter and W2R1 sonority, rs $=-0.26$, $\mathrm{p}<0.001$ for the correlation between the length of the right splinter and W2R2 sonority). The analysis also shows a moderate correlation between the sonority of the boundary phonemes themselves ( $\mathrm{rs}=-0.35, \mathrm{p}<0.001$ for the correlation between W1L1 and W2L2 in the left splinter, and $r s=-0.51, p<0.001$ for the correlation between W2R1 and W2R2 in the right splinter, $r s=0.12, p<0.001$ for the correlation between W1L1 and W2R1 at the switch point of AD-formations). This suggests that the neighbouring sounds are not independent of each other, and confirms that blends are subject to phonotactic constraints, which in itself is not an unexpected finding.

The correlation between the sonority of neighbouring phonemes can be explained by phonotactic constraints that operate for any words, not necessarily blends. Thus, the sonority of a syllable gradually rises from the onset to the peak, and then gradually fades into the coda (see, for example, Giegerich (1992) for a discussion of phonotactic constraints in English). This means that the sonority ranks of any neighbouring phonemes in a word are related, hence the above result. The observed correlation between the sonorities of W1L1 and W2R1 reflects the fact that the left splinter of W1 is often merged with the right splinter of W2 at the boundaries of syllable constituents 
(see the following section for details). This result is compatible to the finding in Kelly (1998), where it was shown that the final phoneme of the left splinter of W1 and the initial phoneme of the right splinter of W2 in blends tend to have similar sonority.
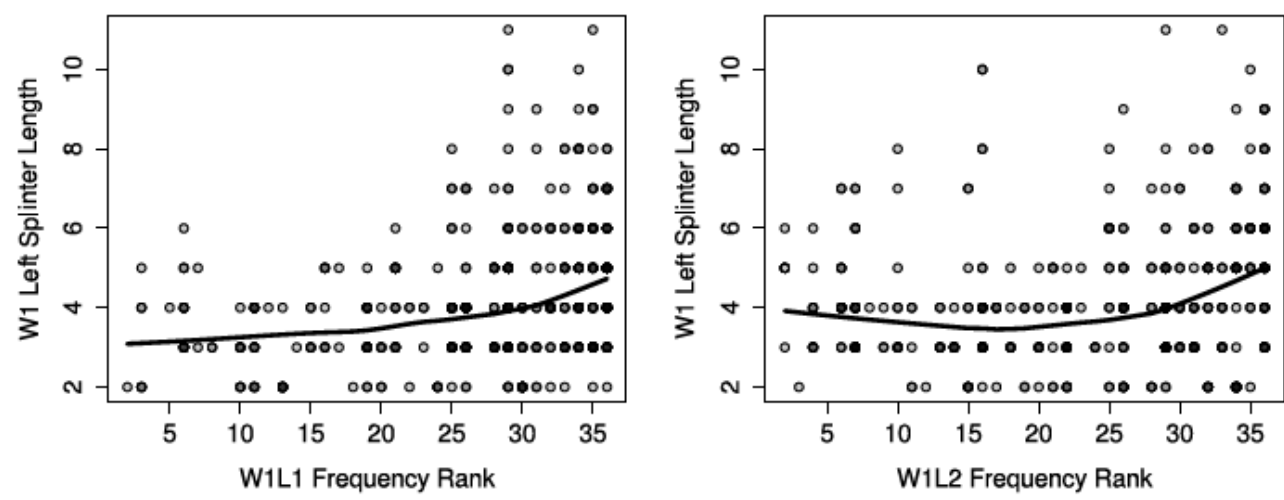

Figure 5. Correlation between the length of the left splinter of the first source word (W1) and the frequency rank of the boundary phonemes. $r=0.33$ for W1L1; $r=0.21$ for W1L2, darker circles represent multiple data points

A more important relation was revealed using frequency ranking of the boundary phonemes. The results of the analysis based on the frequency tables from Mines et al. (1978) are displayed in Figures 5 and 6. The left panel of Figure 5 shows a moderate positive correlation between the length of the left splinter of the first source word, and the frequency of the boundary phonemes W1L1 ( $\mathrm{r}=0.33, \mathrm{p}<0.001)$, the right panel shows a weak $(r=0.21)$ correlation between the length of the left splinter and the W1L2 phonemes, which is also statistically significant $(\mathrm{p}<0.001)$. The correlations illustrate that the initial splinter of a blend is longer if the phonemes at the splinter boundary (W1L1 and W1L2) have higher frequency. Accordingly, lower frequency phonemes tend to be in the positions of W1L1 and W1L2 in shorter splinters. Interestingly, this effect is not observed for the right splinter, i.e. there is no significant correlation between the right splinter length and the frequency of the boundary phonemes $(r=-0.02, p=0.6952$ for the correlation between the right splinter length and W2R1 frequency, $r=0.08$, $\mathrm{p}=0.1027$ for the correlation between the right splinter length and W2R2 frequency, the scatterplots are displayed in Figure 6). 

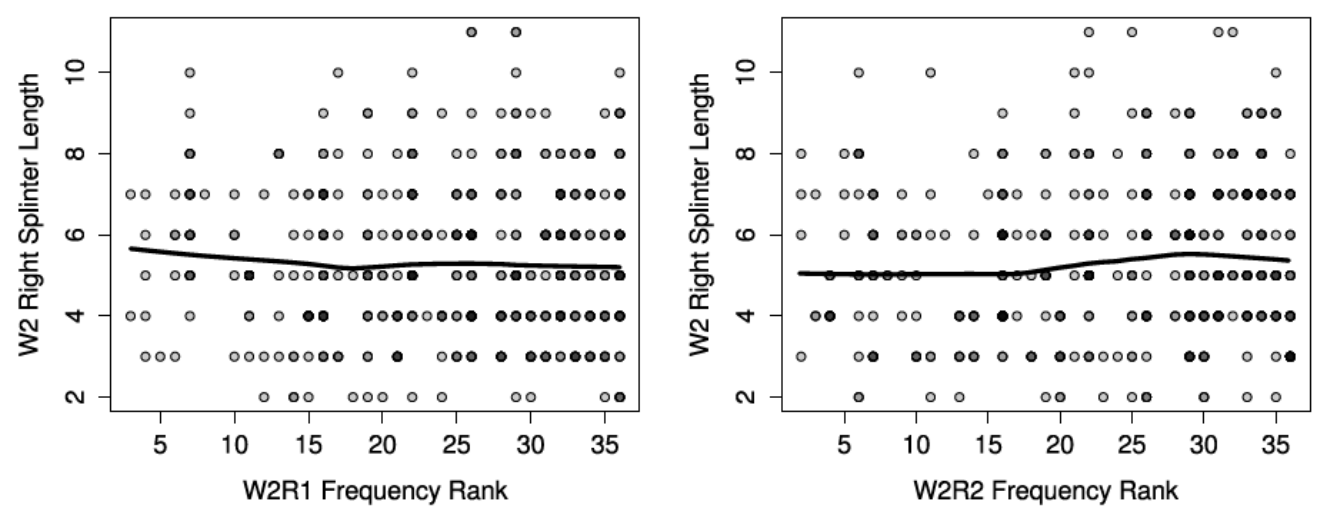

Figure 6. Correlation between the length of the right splinter of the second source word (W2) and the frequency rank of the boundary phonemes.

$r s=-0.02$ for $W 2 R 1$; rs=- 0.06 for W2R2, darker circles represent multiple data points

The analysis of the relationship between the length of the left splinter of the second source word and the frequency rank of the boundary phonemes W2L1 and W2L2 in ACformations like fin-lit (Figure 7) has demonstrated that there is no significant correlation between them ( $\mathrm{rs}=0.2, \mathrm{p}=0.1382$ for the correlation between W2 left splinter length and the frequency of W2L1; rs=0.09, p=0.5041 for the correlation between W2 left splinter length and the frequency of W2L2). The lack of statistical significance of these correlations may be due to the relatively small number of $\mathrm{AC}$ formations in the data $(\mathrm{N}=23$, see Table 3$)$.
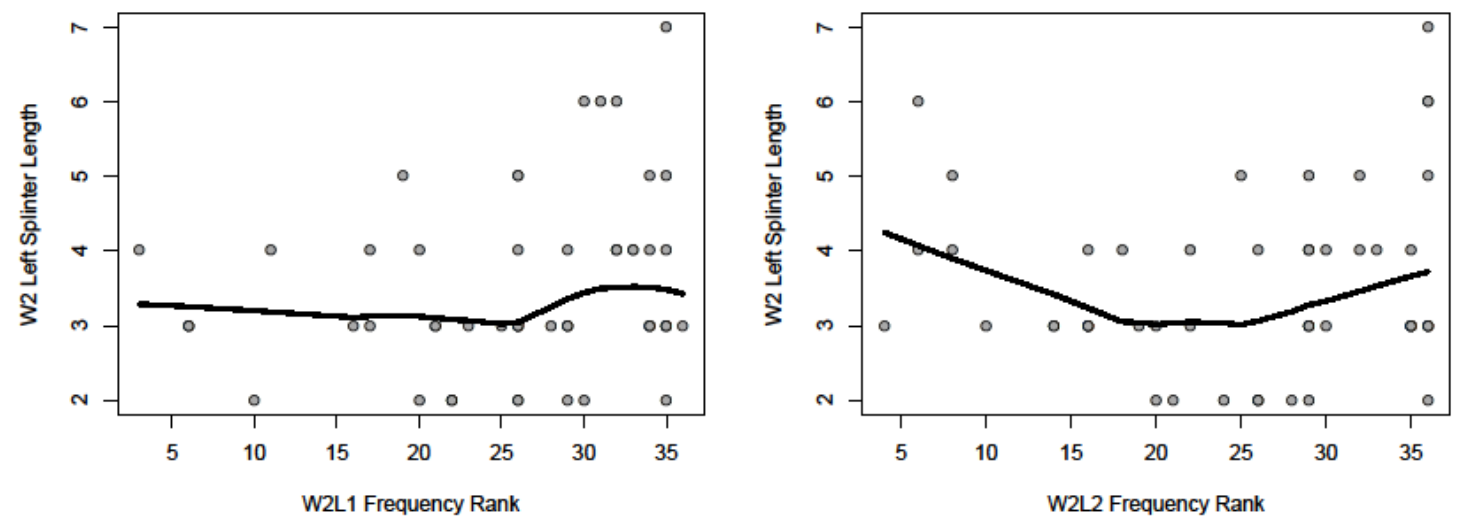

Figure 7. Correlation between the length of the left splinter of the second source word (W2) and the frequency rank of the boundary phonemes in AC formations.

$r=0.2, p=0.1382$ for W2L1; $r=0.09, p=0.5041$ for W2L2, darker circles represent multiple data points

The scatterplot showing the distribution of the values of W2L1 and W2L2 frequency in relation to the length of the left splinter of W2 in Figure 7 is, however, visually more 
similar to the scatterplots in Figure 5 than to those in Figure 6, which suggests that a study of more AC formations may reveal a relationship between the frequency of the boundary phonemes and the length the initial splinter. The observed effect is not simply a natural consequence of phoneme frequency variation (the more frequent the phoneme, the more likely it appears in any given segment of a word), as it is different for the initial and the final splinters.

In sum, the association between the splinter length and the frequency ranking of the boundary phonemes is different for initial and final splinters. This difference can, to an extent, explain the differences between $\mathrm{AD}$ and $\mathrm{AC}$ formations (or blends and clipping compounds) reported, for example, in Gries (2006). It is important, though, to take into consideration the fact that in the above analysis the data were not divided into groups according to the structural types (AC, AD, etc.) and the structural type was not included as a variable. On the one hand, as a result of this approach, the model could not distinguish between, for example, the initial segment $(A)$ in $A D$ blends and in $A C$ formations, which means potentially valuable data were not obtained. On the other hand, differences between different types of splinters are observed nevertheless, which is one of the reasons why it is justified to make this structural distinction. Further analyses in this chapter and also in Chapters 6 and 7 include structural types and aim to detect specific differences between them, in particular, between formations which can be classified as clipping compounds (AC forms) and formations of other structural types.

The fact that frequency is one of the factors determining the 'value' of a phoneme in the formation can be evidence of the relative informativity of the phonemes comprising the source words. That is, the more frequent the phoneme the less information about the source word is 'packed' in it and therefore the more phonemes are needed for the splinter. It is not clear from the above results whether the correlation between the splinter length and the phoneme frequency is observed only in close proximity to the switch point, or throughout the whole word. This is due to the limitation of the analysis described in section 4.2.1, i.e. because only the ranking of the two boundary phonemes in each splinter was considered. What is important for the aims of the present study is that such a relationship is observed in blends, which signifies that the informativity of the constituents influences blend formation. This result, alongside the evidence, for example, from Bell and Plag (2012) concerning informativity as a determinant of 
compound stress, signifies that informativity may work on different levels of word formation.

It is clear that the frequency of the separate phonemes is not the only factor determining the switch point position in a blend: earlier research (e.g Kubozono, 1990; Gries, 2012; Arndt-Lappe and Plag, 2013) has shown the value of other factors. Some of these factors will be considered in the following section.

\subsection{Structural properties: Interaction with phonology}

\subsubsection{Data and methods}

Many studies of the phonology of blends focus on the place of the switch point in their syllabic structure. However, the relations between structural type and prosody (considered here narrowly in terms of syllable structure) have not been taken into consideration. In this section the interaction between the syllable structure of the blends (more specifically, the position of the switch point in relation to the syllable structure) and their structural type will be discussed. It will be considered whether the syllable structure provides grounds for taxonomic differentiation between different structural types, in particular blends and clipping compounds (AC forms).

It is mentioned in the literature (e.g. Kelly 1998; Bauer 2012) that the switch point in blends usually goes either on the syllable boundary or between syllabic constituents, e.g. between onset and rime. The position of the switch point for each blend and clipping compound in the collected corpus was determined with regard to the syllable constituents. It has to be noted, however, than in numerous cases of phonological overlap it is not possible to unambiguously determine the position of the switch point. For example, it is not clear whether the overlapping segment /Iz/ in the blend /blıza:stə/ comes from W1 /blızərd/ or from W2 /dıza:stə/, or indeed from both. That is to say, there is no way to decide whether the two splinters comprising the blend are /bli/ + /zasstə/, /blız/ + /asstə/ or /bl/ + /ızasstə/. To avoid this ambiguity, the phonological content of the splinters and the position of the switch point is determined with regard to all the phonemes from both of the source words that are preserved in blends, including the overlap. In other words, if an overlap takes place there are two possible switch points, one before and one after the overlapping segment. In case of /bliza:stə/, such an analysis results in identifying the right splinter /blız/ with the switch point after /z/, and the left splinter /Izasstə/, with the switch point before /I/. 
Because of this approach to determining splinter boundaries, the position of the switch point(s) is in most cases inseparable from the position of the overlap. Therefore, two possible switch points are accounted for in the analysis.

Depending on whether the full syllables of each source word are preserved or not, all the blends in the collected corpus can be divided into four groups presented in (4.4). In the transcriptions below the parts of the source words which are not retained in blends are in parentheses, the overlapping segments are in bold type, and the syllable boundaries are marked with dots (if not already indicated by stress marks).

a) YY - whole syllables are preserved both from W1 and W2, the switch point is on

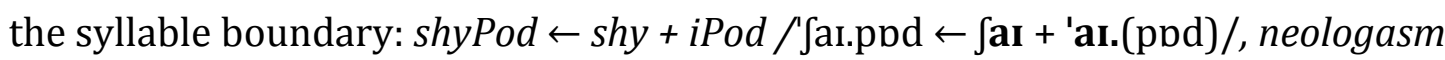
$\leftarrow$ neologism + orgasm /ni'bl.ə.gæz.əm $\leftarrow$ ni'pl.ə.(dzı.zəm) + ('ว:).gæz.əm/;

b) YN - whole syllables are preserved from W1 but not from W2, the switch point is on the syllable boundary of W1, often there is an overlap, e.g. in jewtheran /dzur.Oər.ən/, Jew /dzu: is retained in its entirety, while the syllable /lu:/ in Lutheran /('I)us.Өər.ən/ loses its onset;

c) NY - whole syllables are preserved from W2 but not from W1, the switch point is on the syllable boundary of W2, often there is an overlap, e.g. in microwait /'mar.krəu.wert/, microwave /mar.krəu.wer(v)/ loses its coda, while wait/wert/ is retained in full;

d) $\mathrm{NN}$ - whole syllables are not preserved from $\mathrm{W} 1$ or $\mathrm{W} 2$, the switch point is not on the syllable boundary of either words: chofa $\leftarrow$ chair + sofa /'tfəu.fə $\leftarrow$ t $\int($ eə) + ('s)əu.fə/.

Note that the syllable boundaries for the source words were taken from CEPD and that overlapping segments were coded twice, separately for W1 and W2. In blend formation resyllabification often takes place, and the syllable structure changes. The four groups in (4.4) were formed according to what syllables of the source words are (partially) preserved in the blend, irrespective of whether they are still whole syllables in the blend or they undergo resyllabification. 


\subsubsection{Results and discussion}

Table 5 shows how four of the major structural types of the blends (central replacement blends have two switch points and therefore cannot be compared with the other four types here) are distributed in our corpus in terms of their syllable structure.

It is clear that $\mathrm{AD}$ blends demonstrate a tendency to preserve full syllables from $\mathrm{W} 1$ as in jewtheran in (4.4b). Moreover, over $50 \%$ of blends which preserve full syllables from W1 also preserve full syllables from W2, e.g. wedsite /'wed.sart/ $\leftarrow$ wedding /'wed.(In)/ + website /('web).sait/. Overall, there is a clear tendency to retain full syllables from the beginning of words (which is the case for $246 \mathrm{AD}$ blends, i.e. over $70 \%$ of all AD blends in the data).

Table 5. The syllable structure of the four main types of blends

\begin{tabular}{lllll}
\hline $\begin{array}{l}\text { Syllable } \\
\text { structure of the } \\
\text { blend }\end{array}$ & \multicolumn{2}{l}{ Blend type } & & \\
\cline { 2 - 5 } & AD & AW & WW & AC \\
\hline YY & 131 & 39 & 28 & 6 \\
YN & 115 & 0 & 0 & 6 \\
NY & 26 & 43 & 1 & 6 \\
NN & 60 & 1 & 0 & 5 \\
\hline Total & 332 & 83 & 29 & 23 \\
\hline Grand Total & 467 & & & \\
\hline
\end{tabular}

At first sight it seems that AW blends differ from AD blends in this respect because over half of them are NY, e. g. microwait in (4.4c). However, in AW blends the switch point does not enter the second source word, and therefore the preference to preserve the beginning of the word which can explain this distribution.

For WW blends full preservation of the source words results in preservation of full syllables from both of them apart from homoblivious $\leftarrow$ homo + oblivious

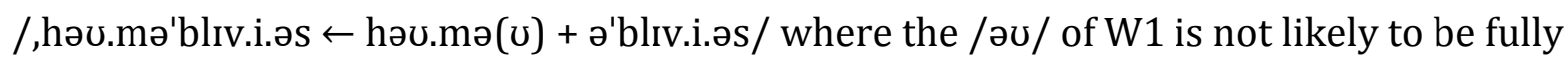
pronounced and hence only schwa vowel was included in the transcription of the blend.

In case of clipping compounds it is by definition the beginning of the word which is preserved in all cases, therefore this factor is a defining one for this group. It is also 
worth noting that no clear preference for any of the four situations in terms of syllable preservation is observed for AC formations.

In the YY group, the position of the switch point is always on a syllable boundary. This includes cases with overlap, in which both potential switch points fall onto syllable boundaries. In YN and NY groups the switch point (or one of the two switch points, in cases of overlap) was found in the following positions:

a) between onset and nucleus (119 observations), e. g. in W2 of blizzaster /blı'za:.stə/ $\leftarrow$ blizzard /'blız.(ərd)/ + disaster /(d)ı'za..stə/;

b) between nucleus and coda (65 observations), e. g. both in W1 and W2 of

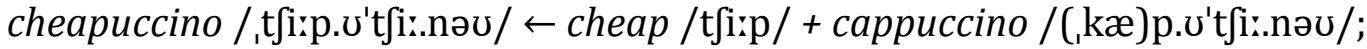

c) within onset (8 observations), e. g. in W1 of awkfest /'o:k.fest/ $\leftarrow$ awkward /'כr.k(wəd)/ + fest /fest/;

d) within coda (1 observation) in W1 of frienvy /'fren.vi/ $\leftarrow$ friend /fren(d)/ + envy /'en.vi/.

A conditional inference tree (decision tree) analysis was performed to figure out if the switch point placement is related to the structural type of blends. The method involves estimating a regression relationship between the variables by binary recursive partitioning in a conditional inference framework. In the process of building a decision tree, the dependent variable is analysed in relation to one or several independent variables. First, the algorithm tests the null hypothesis of independence between the dependent variable and any of the independent variables. If the null hypothesis is rejected, the independent variable which has the strongest association with the dependent variable is selected. At this stage, the data is split into two groups (branches) if the difference between the value of the outcome variable in two branches 'growing' from one node is statistically significant at the $5 \%$ level (that is, the p-value must be smaller than 0.05 in order to split the node). Then this process is recursively repeated until further splits are no longer justified. Each time the full set of independent variables is taken into consideration for a potential node split, so that the same variable can cause more than one split (see Hothorn et al. (2006) for a detailed description of the method). 
In the following analysis, the position of the switch point and phonological overlap were used as independent variables, and the structural type of formations (AD, AW, WW and AC) as the dependent variable. A decision tree was built for each of the groups: YY, NN, YN and NY. As a result, the only group in which a significant effect of the independent variables on the type of the formation was detected is YN. The trees built for YY, NN, and NY groups did not show any splits between the nodes which means that for that part of the data the structure of the blend or clipping compound did not appear to be the outcome of the switch point position and overlap. The outcome of the decision tree analysis for the YN group (consisting of $115 \mathrm{AD}$ and $6 \mathrm{AC}$ forms, see Table 5) is shown in Figure 8, the exact p-values are displayed in the node labels.

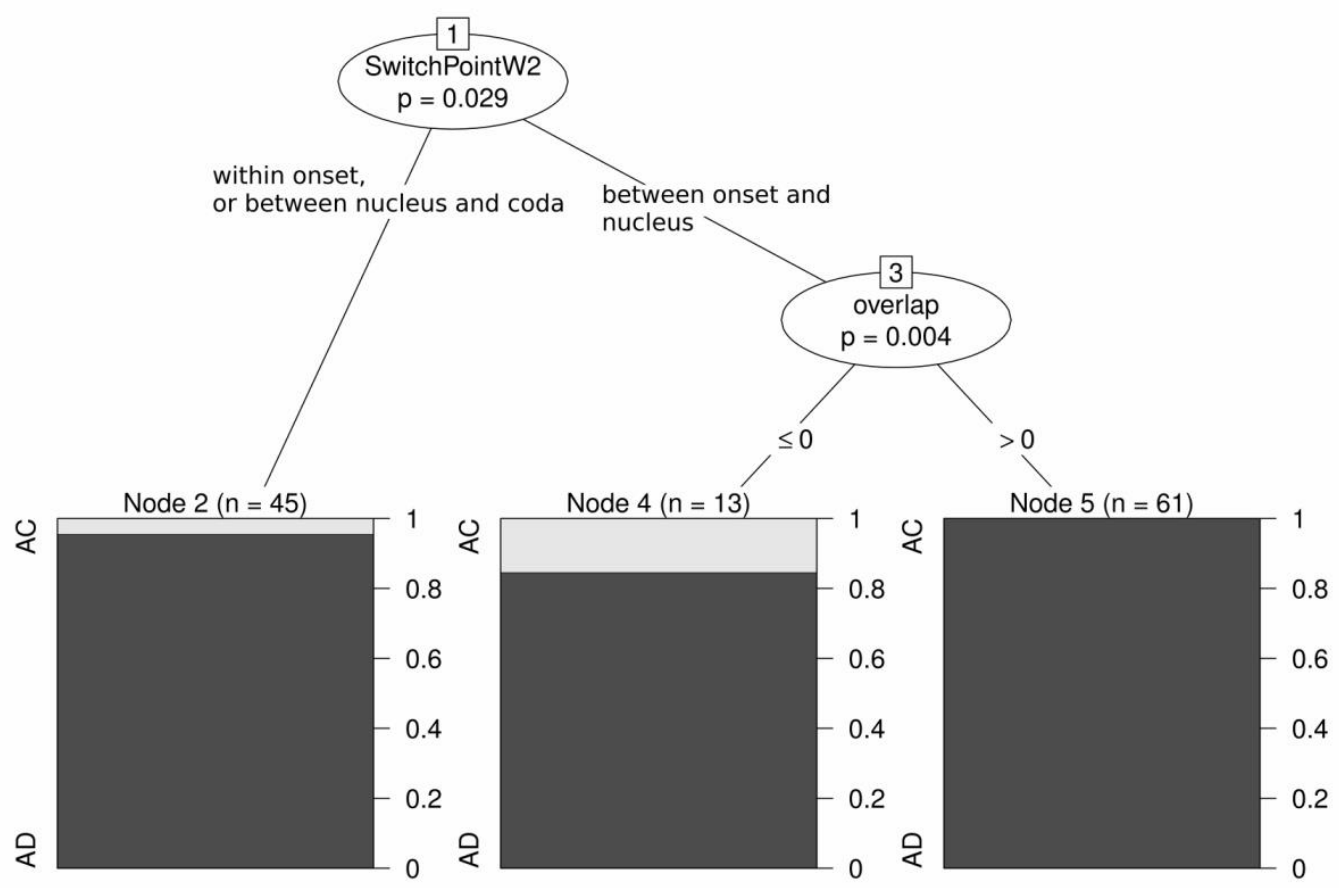

Figure 8. The results of the decision tree analysis of the influence of the switch point placement and the phonological overlap on the structural type of a blend in the YN group

One of the distinguished nodes (node 2), in which the switch point is placed either within onset or between nucleus and coda, contains $42 \mathrm{AD}$ blends and $3 \mathrm{AC}$. Among the blends where the switch point is placed between onset and nucleus, two nodes are distinguished (nodes 4 and 5): with an overlap consisting only of AD blends, and the group of non-overlapping blends containing $3 \mathrm{AC}$ forms and $10 \mathrm{AD}$. It appears from the outcome of the decision tree that $\mathrm{AC}$ formations are distinguished from $\mathrm{AD}$ blends by lack of overlap. In sum, AC formations deviate from the rest of the data in two aspects: 
first, they do not tend to preserve whole syllables from their source words, second, their source words do not phonologically overlap.

In Gries (2006) it was stated that clipping compounds behave differently in terms of recognisability of their source words; mainly, the switch point falls earlier than is necessary for the source words to be easily recognisable by their phonological and orthographical material. The results above confirm that the formation of AC does not involve the same phonological constraints as the formation of blends. How exactly it is related to the recognition of the source words will be further explored in Chapters 6 and 7.

It is essential to bear in mind that recognisability works differently depending on whether the initial or the final segment of a source word is included in the blend. More discussion of the factors which are important for word recognition will follow in Chapter 5, so here I will only make a note of what is essential for the present analysis. As mentioned, for example, in Whitney (2001), word beginnings are remembered more easily than word endings or middle parts. On the other hand, in addition to the graphemes and sounds per se, a word can be recognised by its rhythmic pattern (Gries, 2006). The number of the phonemes (or, to be more precise, syllabic constituents) that are preserved from the beginning of each source word is determined by how many of them are sufficient for the source word to be recognisable. AC forms differ from other structural types in this respect because the switch point is positioned: 1) relatively early; 2) differently within their syllabic structure. If the end of the word is preserved, the main stress position and the overall prosodic structure of the word become important for recognition. Cannon (1986) observes that blend words tend to retain the main stress of one or both of their source words. Recent studies, for example, Bat-El and Cohen (2012), have revealed, more specifically, that the stressed syllable of the second source word is more likely to be preserved in a blend than the stressed syllable of the first source word. An OT analysis of experimentally induced blends in Arndt-Lappe and Plag (2013) has shown that even if the stressed vowel, or even all the phonemes of the stressed syllable are not retained in the blend, the prosodic structure, that is, the number of syllables and the main stress position of the second source word tends to be preserved.

The studies above discuss the preservation of the prosodic pattern in blends which combine the beginning of one word with the end of another, that is, according to the 
present classification, in AD blends only. A decision tree analysis was carried out to check whether there is a difference between AD blends and other formations in the present corpus in terms of preserving the prosodic pattern of their source words. The prosodic pattern was defined, following Arndt-Lappe and Plag (2013), as the overall number of syllables plus the main stress position.

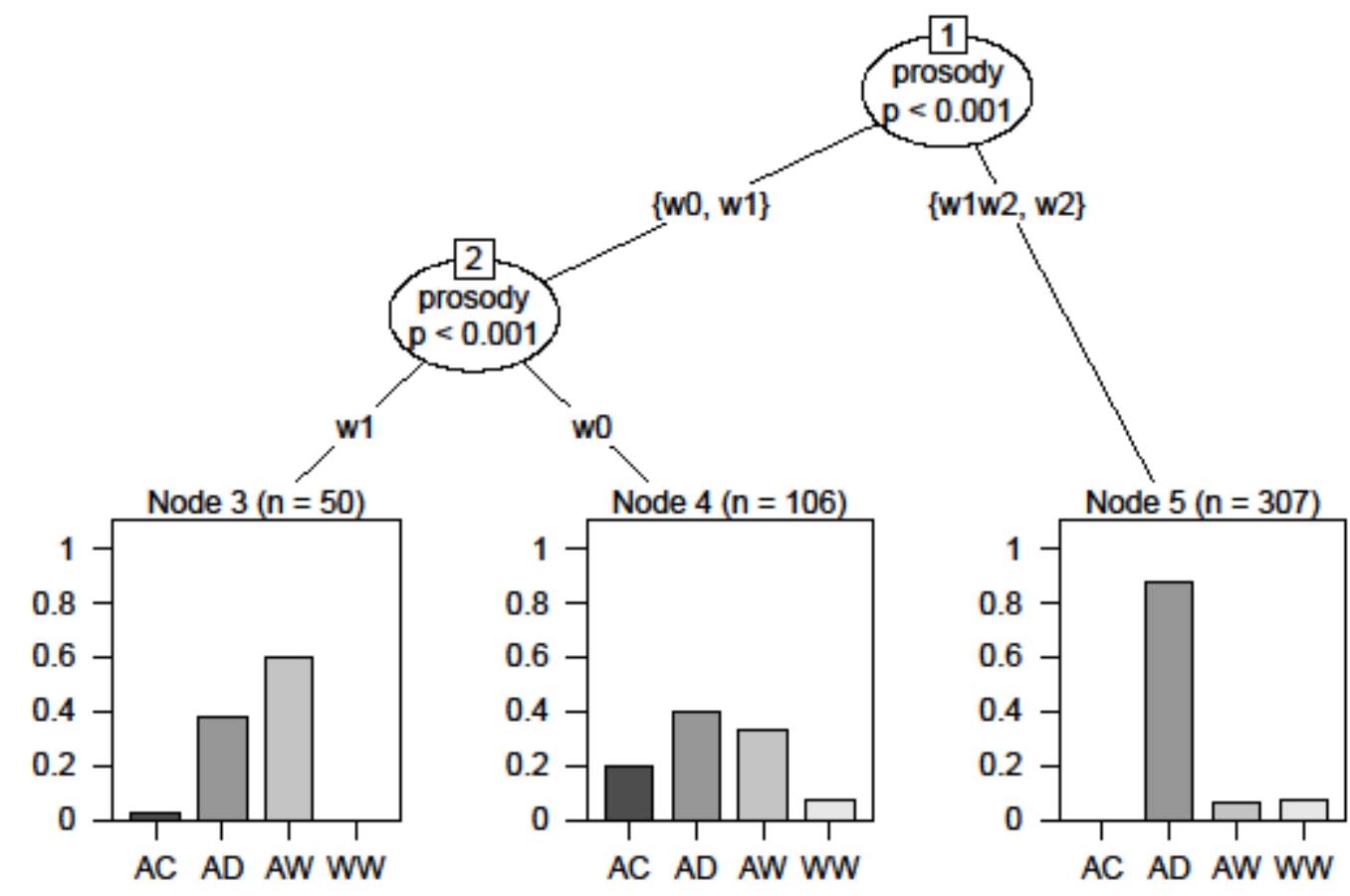

Figure 9. The prosodic pattern of source words repeated in a blend as the predictor of the structural type of the blend

For the decision tree displayed in Figure 9, the prosodic pattern of a formation was used as an independent variable, and the structural type as the dependent variable. The node labels w1 and w2 in Figure 9 stand for reproducing the prosodic structure of W1 and W2 respectively. The label w1w2 means that the prosodic pattern of both the source words is preserved, which is the case for $20 \mathrm{AD}$ blends and $1 \mathrm{AW}$ blend, 9 of them monosyllabic (e.g. shress $\leftarrow$ shirt + dress). The prosodic structures of blends which are different from those of both the source words were labelled as w0. As seen in Figure 9, AD structural type is over-represented in node 5 , i.e. the majority of AD blends follow the prosody of W2, or preserve the prosody of both their source words. AW forms are over-represented in node 3 , that is, the majority of forms that reproduce the prosodic structure of W1 are of AW type, although a considerable number of AW do not preserve the prosodic structure of any of their source words (node 4). No WW blends reproduce the prosody of W1 (no WW forms in node 3), but no clear tendency towards preservation of W2 
prosody in WW can be determined either, as they are split between node 4 and node 5 . AC is the only structural type which almost categorically appears in one node (node 4), which indicates that almost all AC forms do not follow the prosodic pattern of any of their source words.

In sum, phonological differences between blends and clipping compounds have been revealed at different levels: at the level of phonemes, syllable constituents, and the word-level prosodic structure.

\subsection{Semantic properties: Interaction with structure}

\subsubsection{Data and methods}

The semantic properties of blends have often been used as the basis for their classification according to the relationships between their source words, similar to the classifications of compounds according to the semantic relationships between their components, e.g. in Downing (1977), Bauer (1983), Benczes (2006) and Renner (2008). In some cases particular semantic properties were used as distinguishing features of blends. For example, in Adams (1973), Berg (1998), and Kelly (1998) a word is classified as a blend (or at least as a typical blend) only if its source words are in some kind of coordinative relation, e.g. synonymic or antonymic, or are hyponyms, otherwise it is a clipping compound. Making this distinction seems, however, no less arbitrary then a similar distinction based on purely formal properties of blends and clipping compounds, as was shown in section 2.2. An integrative approach to this problem might help find more reliable grounds for distinguishing and classifying blends. In this section, the interaction between the form and the semantics of blends will be considered. An attempt to make a subtle classification of semantic types and subtypes of blends is not among the aims of the present research, therefore only two main semantic types were taken into consideration, based on the classification from Bauer (2006):

1. paradigmatic origin blends (chofa $\leftarrow$ chair + sofa, blizzaster $\leftarrow$ blizzard + disaster $)$;

2. syntagmatic origin blends (fake-ation $\leftarrow$ fake vacation, briet $\leftarrow$ bride diet).

These terms are synonymous to the terms 'coordinative/determinative blends' used, for example, in Bauer (2012). Referring to the blends as having either paradigmatic or syntagmatic origin implies looking at not only the semantics, but also the origin of the blends. Paradigmatic origin blends can be glossed by linking their source words with an 
and or or (chofa has properties of a chair and a sofa). Syntagmatic origin blends can be glossed by modifying the second source word by the first one (e.g. briet is a bride diet, i.e. a kind of diet). It is possible that a blend of either kind actually originates from an itemfamiliar word combination of the corresponding type, but it is not necessarily the case. Defining the semantic type of neologisms in my collection relies on the definitions and the source words provided in the sources, and on the semantic relations between the source words analysed in accordance with the criteria in Bauer (2006). Thus, the data are distributed into the semantic categories in the following way: 391 formations have syntagmatic origin, 109 have paradigmatic origin, and the remaining 6 are classified as 'other'. The latter group includes formations which are problematic to assign to either semantic type because the order of the source words in the explaining word combination is reversed, as in epiphanot $\leftarrow$ not an epiphany, or is questionable, as in collelephant $\leftarrow$ college + elephant, meaning 'a large college'. Although one might argue that the source words of collelephant are in subordinative relations, this blend is still different from other subordinative origin blends. If we assume that it is a subordinative blend parallel to others, this would imply that the second source word is the head word, i.e. the meaning would be something like "an elephant with a college-like property" which is not what the context demands.

Combinations of source words, i.e. cases of their immediate co-occurrence, were looked up in COCA for each blend or clipping compound. Irrespective of the semantic type of the blend or clipping compound in question, both subordinative and coordinative combinations of its source words were looked up. The subordinative word combinations could have only one possible word order, which was determined by the meaning of the blend, and the coordinative word combinations could have different order of constituents. In coordinative word combinations, the source words could be conjoined by and or or, or could have a comma or a hyphen between them; all these variants were looked up in the corpus.

\subsubsection{Results and discussion}

For about one third of the data (149 of 506 lexemes) the source word combinations could be found in COCA, and in the vast majority of cases only one type of combination was attested, particularly, coordinative (either in direct or in reversed order, or both) for paradigmatic origin formations, and subordinative for syntagmatic origin formations (the exact numbers are given in Tables 6 and 7). 
For 54 of 109 (49.5\%) blends of paradigmatic origin, coordinative source words combinations are attested in COCA. As for syntagmatic origin blends, only $18.9 \%$ of the subordinative combinations of their source words (74 out of 391) were found in COCA. The observed difference is higher than could be suggested by chance $(p<0.01$ for a t-test of the difference of proportions).

Table 6.The distribution of the data with regard to the semantic origin and the type of source word combinations attested in COCA

\begin{tabular}{|c|c|c|c|c|c|c|}
\hline \multirow[t]{3}{*}{$\begin{array}{l}\text { Semantic } \\
\text { type of } \\
\text { formation }\end{array}$} & \multirow[t]{3}{*}{$\begin{array}{l}\text { Number } \\
\text { of types } \\
\text { in the } \\
\text { collection }\end{array}$} & \multicolumn{5}{|c|}{$\begin{array}{l}\text { The combination of source words attested in } \\
\text { COCA } \\
\text { (number, \% among all formations of the given } \\
\text { semantic type) }\end{array}$} \\
\hline & & \multirow[t]{2}{*}{ Subordinative } & \multicolumn{3}{|c|}{ Coordinative } & \multirow[t]{2}{*}{ None } \\
\hline & & & $\begin{array}{l}\text { In direct } \\
\text { order }\end{array}$ & $\begin{array}{l}\text { In reversed } \\
\text { order }\end{array}$ & Total & \\
\hline $\begin{array}{l}\text { Syntagmatic } \\
\text { origin }\end{array}$ & $\begin{array}{l}391 \\
(100 \%)\end{array}$ & $\begin{array}{l}74 \\
(18.9 \%)\end{array}$ & $\begin{array}{l}22 \\
(5.6 \%)\end{array}$ & $\begin{array}{l}11 \\
(2.8 \%)\end{array}$ & $\begin{array}{l}18 \\
(4.6 \%)\end{array}$ & $\begin{array}{l}302 \\
(77.2 \%)\end{array}$ \\
\hline $\begin{array}{l}\text { Paradigmatic } \\
\text { origin }\end{array}$ & $\begin{array}{l}109 \\
(100 \%)\end{array}$ & $\begin{array}{l}20 \\
(18.3 \%)\end{array}$ & $\begin{array}{l}46 \\
(42.2 \%)\end{array}$ & $\begin{array}{l}41 \\
(37.6)\end{array}$ & $\begin{array}{l}54 \\
(49.5 \%)\end{array}$ & $\begin{array}{l}51 \\
(46.8 \%)\end{array}$ \\
\hline Other & $\begin{array}{l}6 \\
(100 \%) \\
\end{array}$ & $\begin{array}{l}1 \\
(7.8 \%) \\
\end{array}$ & $\begin{array}{l}1 \\
(7.8 \%) \\
\end{array}$ & $\begin{array}{l}0 \\
(0 \%) \\
\end{array}$ & $\begin{array}{l}1 \\
(7.8 \%) \\
\end{array}$ & $\begin{array}{l}4 \\
(66.7 \%) \\
\end{array}$ \\
\hline Total & $\begin{array}{l}506 \\
(100 \%)\end{array}$ & $\begin{array}{l}95 \\
(18.8 \%)\end{array}$ & $\begin{array}{l}69 \\
(13.6 \%)\end{array}$ & $\begin{array}{l}52 \\
(10.3 \%)\end{array}$ & $\begin{array}{l}73 \\
(14.4 \%)\end{array}$ & $\begin{array}{l}357 \\
(70.5 \%)\end{array}$ \\
\hline
\end{tabular}

Table 7. Source words combinations extracted from COCA

\begin{tabular}{|c|c|c|c|}
\hline $\begin{array}{l}\text { Type of source } \\
\text { word combination }\end{array}$ & $\begin{array}{l}\text { Number of types in } \\
\text { the data set for } \\
\text { which this type of } \\
\text { combination was } \\
\text { found }\end{array}$ & $\begin{array}{l}\text { Number of tokens } \\
\text { in COCA }\end{array}$ & Examples \\
\hline coordinative & 69 & 11676 & chair and sofa \\
\hline $\begin{array}{l}\text { coordinative, in } \\
\text { reversed order }\end{array}$ & 52 & 5550 & sofa and chair \\
\hline subordinative & 95 & 1664 & fake vacation \\
\hline
\end{tabular}

Moreover, the decision tree analysis with frequency and type of source word combinations as independent variables predicting the semantic type of the formation shows (Figure 10) that the frequency of the subordinative combinations of the source words does not affect the probability of forming a blend of them (this is why it is not shown in the decision tree although it was included in the analysis as an independent 
variable), while the frequency of coordinative combinations affects the probability of paradigmatic blends. Blends of this kind are more likely to be formed if the corresponding word combinations are attested (nodes 4 and 5 in Figure 10) than otherwise (node 3). This is an important piece of evidence for the practicability of distinguishing between neologisms of syntagmatic and paradigmatic origin.

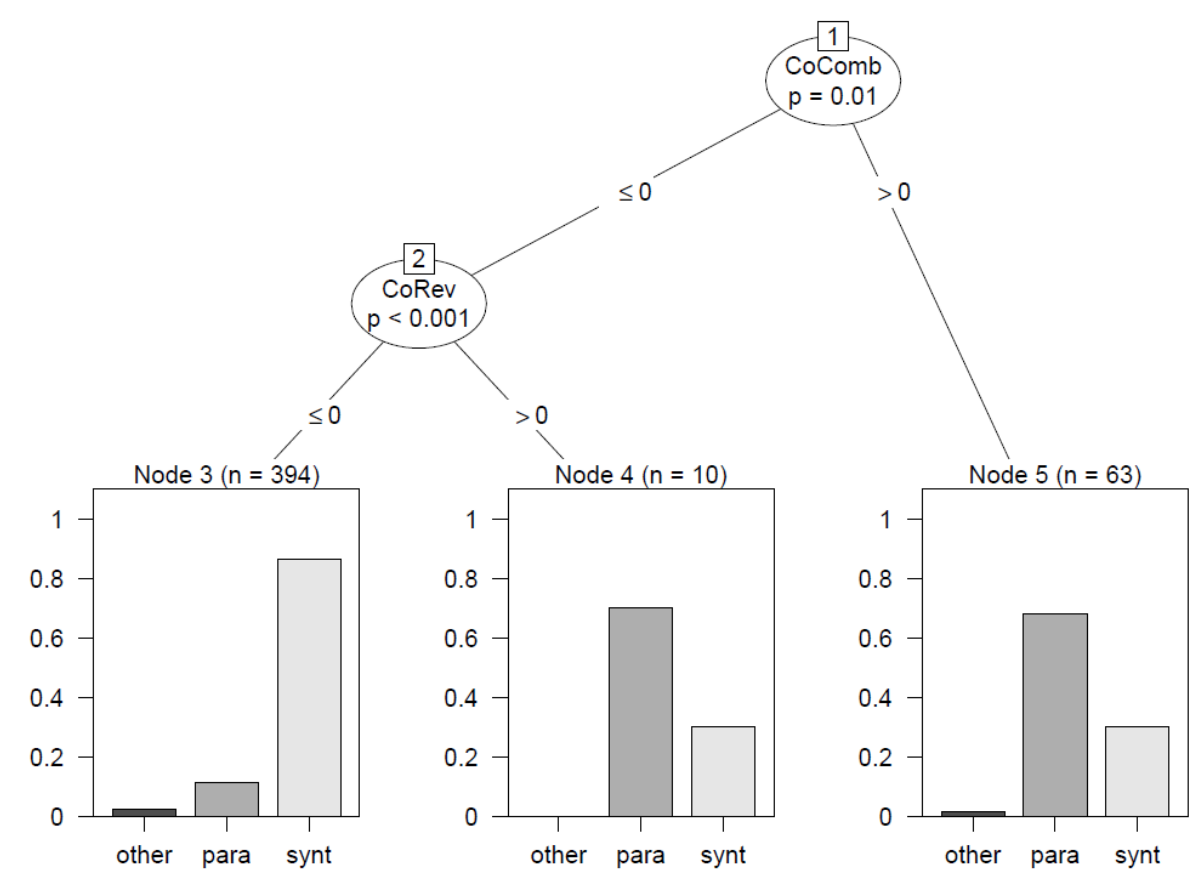

Figure 10. Frequency and type of source word combinations as the predictor of the semantic type of blends.CoComb-COCA frequency of the coordinative source word combination in direct order;

CoRev - COCA frequency of the coordinative source word combination in reverse order

Consider now the structural type of a blend in relation to its semantics. The analysis below is focussed on four structural types: AC, AD, AW and WW. The majority of neologisms of all structural types is of syntagmatic origin, the proportion of syntagmatic origin formations of three structural types being roughly the same (75.4\% of AD forms, $75.9 \%$ of $\mathrm{AW}$ and $77.3 \%$ of AC forms) and higher (89.7\%) for WW blends. In terms of the proportions of the lexemes whose source word combinations are attested in COCA, two structural types differ from the others, as shown in (4.6): for WW blends it is considerably lower and for AC formations considerably higher than for the remaining two types.

$$
\text { AD - 96/332 (28.9\%) }
$$




$$
\begin{aligned}
& A W-28 / 83(33.7 \%) \\
& W W-2 / 29(6.9 \%) \\
& A C-11 / 23(47.8 \%)
\end{aligned}
$$

Coincidentally or not, these are the structural types which differ from the rest of the data in terms of the degree of preservation of the source words. WW blends, e.g. predictionary $\leftarrow$ prediction + dictionary, stoption $\leftarrow$ stop + option, fully preserve their source words due to the overlap, and AC formations preserve a relatively small portion of the phonological and graphical material of their source words (as shown in 4.2.1.). This, in turn, results in lower potential for recognisability of the source words from an AC form, as discussed in Chapter 2.

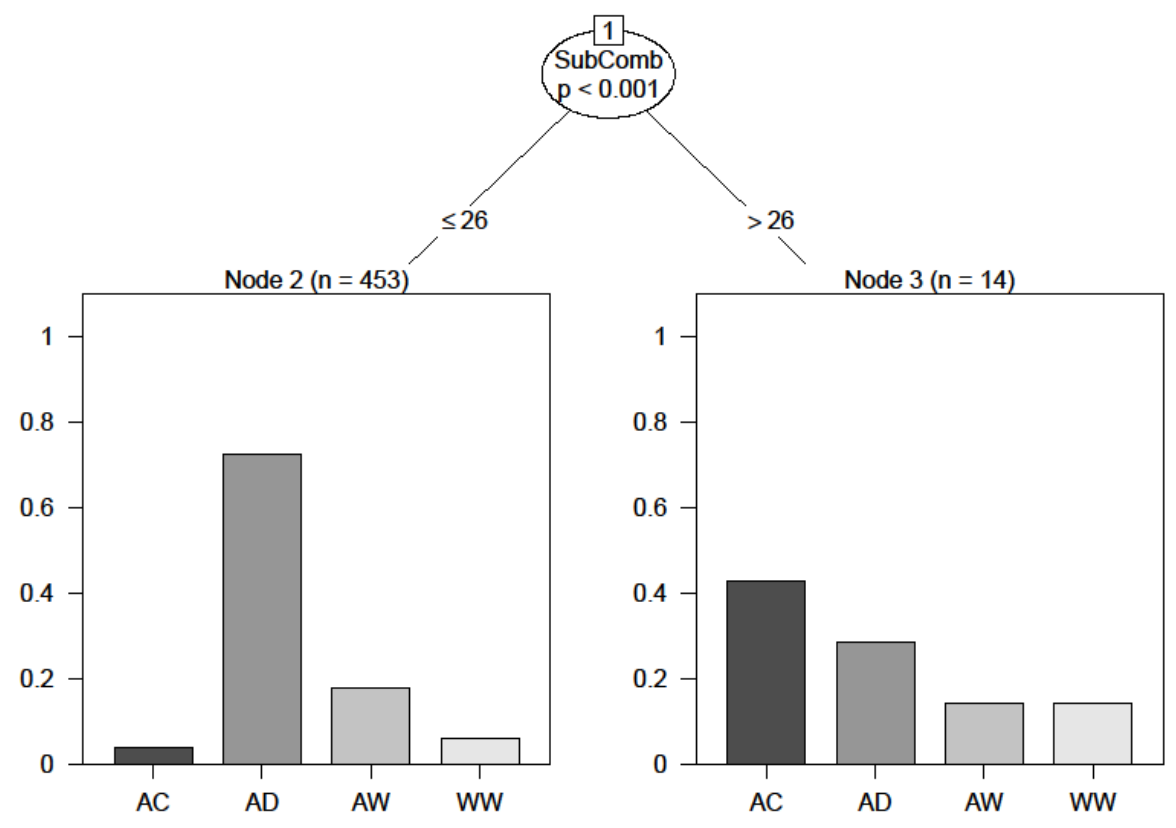

Figure 11. Frequency and type of source word combinations as the predictors of the structural type of blends. SubComb-COCA frequency of the subordinative source word combination

Thus, WW blends seem to be formed using completely different principles, rather than merging together words that are frequently encountered side by side. As for AC formations, an opposite tendency is observed. The recognisability of the source words seems to be of low priority for the formation of these blends because of the different principles of the switch point placement. AC formations demonstrate a tendency to be coined out of words that are encountered together. As the decision tree in Figure 11 shows, the proportion of AC forms is significantly higher in node 3 than in node 2 . The 
subordinative source word combination frequency for node 3 is over 26 , while the frequency of the subordinative source word combinations of blends in node 3 is lower. This means that a complex formation merging together two words is significantly more likely to take the AC form if the frequency of the corresponding subordinative source word combination exceeds 26 . This result implies that an AC form is more likely to be formed as a contraction of an established word combination (i.e. a clipping compound in the sense that it is a clipping of an existing compound) than as a neologism naming a completely new notion. The latter is, for example, the case with hybrid names such as chofa $\leftarrow$ chair + sofa.

If the semantic relationships between the source words are used for the classification of blends among other morphological processes, then blends are often juxtaposed to contractions of 'words which occur side by side', as formulated in Kubozono (1990, p. 2). For example, Gries (2012, p. 155) reports evidence that 'complex clippings have quite a strong preference to involve contractive relations', which means that, unlike blends, complex clippings (AC formations here) tend to merge together words which could appear as a compound. The analysis of the interaction of the structure of the formations and the semantic relations between their source words shows that the structural type of a neologism is indeed related to its actual origin.

\subsection{Interim conclusions: Phonological and semantic factors which influence the structure of blends}

The coinage of blends does not employ morphemes but involves extraction of segments of the source words and merging them together following prosodic rules, which determine the position of the switch point. The findings described in sections 4.2-4.4 reveal the factors that influence the switch point placement: the sonority and frequency of boundary phonemes, and the syllable structure of the source words. The correlation between the sonority ranks of the phonemes adjacent to the switch point indicates that blend words are formed in accordance with the phonological constraints for English words. The positive correlation between the frequency of boundary phonemes and the splinter length can be explained by the recognisability of the source words, which has been shown to be an important factor in blend formation (Gries 2004, 2006, 2012). The frequency of the phonemes, as an indirect indicator of their informativity can be related to recognisability of the source words from the splinters in blends and clipping compounds. This study has shown that in terms of the switch point position AC 80 
formations behave differently from AD blends. Firstly, they preserve less material from the source words than $\mathrm{AD}$ (or any other type of formations considered in the analysis above). In addition to this, AC forms demonstrate a clear preference for the switch point to be placed between onset and nucleus in the situation of no phonological overlap, whilst $\mathrm{AD}$ blends do not show any preference in this respect at all. Although on the surface (considering only the phoneme sequences) the phonology of both $\mathrm{AD}$ and $\mathrm{AC}$ forms is similar to the phonology of other morphological categories, e.g. monomorphemic English words, the picture becomes different if we take into consideration the position of the switch point in the syllabic structure and the factor of recognisability of the source words.

Another factor which must be taken into consideration in an analysis of the structure of blends and clipping compounds is the prosodic contour of their source words. The analysis in section 4.3 shows that whether the stress pattern of the first or the second source word (or of none at all) is preserved, is not independent of the structural type of the formation. The majority of AD forms preserve the prosodic pattern of the second source words, which is compatible with earlier findings in literature. Unlike AD, AC forms tend not to preserve the prosodic pattern of any of their source words. The fact that AW blends tend to follow the prosodic pattern of W1 rather than $\mathrm{W} 2$ can be explained by purely technical reasons. The first source word of AW blends usually has more syllables than the second (or, to put it the other way round, W2 is fully preserved in these blends due to the fact that it is short, usually mono- or disyllabic), e.g. passthought $\leftarrow$ password + thought. Therefore it is impossible for the whole blend to reproduce the prosodic structure of the second source word. Besides, as the second word is, by definition, fully preserved in AW blends, it ensures its recognisability and makes its prosodic structure less important for recognition. It must be noted, however, that a small group of AW blends, e.g. enviclean $\leftarrow$ environment + clean do not follow these tendencies and resemble AC formations in this respect.

It appears that two contradictory factors influence the formation of blend words. The first is the relatively frequent co-occurrence of the source words and, therefore, the possibility of an established semantic link between them. The second factor is the creation of such a link simultaneously with the formation of a blend, which requires a high degree of recognisability of both the source words. Recognisability is not so vital in the first case because the semantic link is already there and the main aim of the 
formation of a new lexeme is merging the source words together in a compact form. The first factor, therefore, is responsible for producing clipping compounds, the second is responsible for blending.

This model can explain the differences in the formation of blends and clipping compounds. Recognisability of the source words is achieved by balancing the preservation of the longest possible segment of both the source words (an ideal case would be a WW blend) and the prosodic pattern of at least one of them. Which prosodic pattern is preserved depends on the relative length of the source words and the blend, the position of the source words (the second source word is more vulnerable if it loses its beginning, so it is essential to preserve its prosodic pattern) as well as on which of them is more valuable for the semantics of the blend. Thus, if one of the source words is the semantic head, it may be the cause for the whole blend to reproduce its prosody, the default pattern for English blends being the preservation of the second source word prosodic pattern. The results of the experiments reported in Shaw et al. (2014) show that, at least for blends labelled here as $\mathrm{AD}$, this is indeed the case. The experiments by Shaw et al. demonstrate that AD blends which can be analysed as right-headed tend to preserve the prosodic pattern of the right-hand source word to a greater extent than paradigmatic origin $\mathrm{AD}$ blends, which do not have a semantic head. In the present study, this works for AD blends and the majority of AW but WW and AC forms behave differently.

As for AC forms, recognisability seems to be a less important factor for their formation than for AD blends. A detailed discussion of the matter and statistical evidence of the differences between the two categories in terms of the recognisability and the similarity of the source words is provided in Gries $(2006,2012)$. This study finds explanation for these differences in the fact that $\mathrm{AC}$ forms originate as an instance of shortening which often implies the existence of a frequently used combination of source words and the existence of a certain semantic link between them prior to the formation of the new lexeme. I tried to filter out such cases at the stage of data collection (see section 4.1), but the analysis has shown that this might be the defining feature of AC forms.

WW blends are on the opposite end of the axis from AC forms. In WW blends, the conditions for the recognisability of the source words are met more successfully than in any other structural type of blends, because both words are preserved in full. The frequent co-occurrence of the source words of these blends is, on the other hand, the 82 
least probable case. A possible explanation of these features of WW blends is that they are an instance of creative word formation introducing a new cognitive unit. To what extent this is true, and whether this also relates to AD blends will be explored in the following chapters.

Contrary to the claim, for example, in Tomaszewitcz (2012, p. 221) that "no relationship between the phonological structure and syntactic origin is assumed to exist in the English blends", the analysis that involves AD, AW, AC and WW structural types shows that the origin has its explanatory value. Considering the origin of a blend alongside its structure, it is possible to distinguish two groups of AW forms, some of them likely to be formed as blends (e.g. approximeeting), others as clipping compounds (e.g. enviclean, see also contrail $\leftarrow$ condensation + trail, lumist $\leftarrow$ luminous mist in Tomaszewitcz $(2012$, p. 228)), although some cases still may be debatable.

The results of this research make an important contribution to the resolution of a much discussed problem: whether clipping compounds and blends are the same type of word formation or not. In terms of purely formal or purely phonological features these two groups of words demonstrate different behaviour, and the reason for this lies in their semantic properties. AC-forms seem to appear contractions of existing compounds, and therefore can be labelled as clipping compounds. Unlike AC-forms, other blends are indeed more likely to be instances of creative word formation involving the formation of new notions in the process of conceptual integration. In other circumstances, a blend of digital and camera could be digamera, but because it was probably coined as the shortening of already established digital camera it took the form of a clipping compound digicam.

Going back to the different approaches to classifying blends as a word formation type given in the Introduction, I have to note that to call blends either an instance of shortening or an instance of compounding would be imprecise, as the process of blend formation appears to be more complex. It is likely that blends or clipping compounds are formed in one of two possible situations: either pure shortening takes place (in most cases after compounding) which results in the formation of clipping compounds, or shortening and compounding happen simultaneously. In both cases phonological rules apply, but in the second case the output word not only has to sound like a normal English word, but also has to preserve enough material from its source words for them to be recognisable. This is achieved by preserving a certain amount of the phonological 
material from the source words, as well as prosodic patterns of the appropriate source word. This leads to a conclusion that both formal criteria that were stated in the working definition in section 3.1 (i.e. partial loss of the phonological/graphical material and not being formed by concatenation of morphs) are important for distinguishing blends from the neighbouring word formation categories.

Applying these criteria to the corpus of data which was analysed in the present chapter leads to a somewhat paradoxical conclusion: blends and clipping compounds are definitely not the same because they have different reasons for appearing and morphologically (if this word indeed can be applied to their formation) they are formed according to different principles. Yet the ultimate boundary between the two categories is impossible to draw because there are, however few, marginal cases the formation of which may be equally successfully explained by either principle.

It has to be noted with regard to the results presented here that some of the conclusions (in particular, the analysis of the syllable structure of the data in section 4.3) are based on small data samples, and therefore should be treated with caution. Despite these limitations, the results presented in this chapter provide important evidence of the influence on the co-occurrence of constituents on the form of the output items. The relations between the structural type of the items and the recognisability of their source words are further explored in two experimental studies described in Chapters 6 and 7. Some ways to provide further confirmations to the claims made here will be suggested in the following chapter. 


\section{Chapter 5. Deconstructing blends: Insights from psycholinguistic and cognitive studies}

Studying phonological and structural regularities of blends and related morphological phenomena consistently leads to the questions of 1 ) how blends are constructed from the point of view of their coiners, and 2) how the readers or hearers of blends perceive and understand them. As is clear from Chapter 2, these questions have often been raised by researchers conducting descriptive and corpus-based studies of blends within various theoretical frameworks. The findings of the lexical data analysis presented in Chapter 4 also reveal that the differences between structural types of blends and clipping compounds are related to factors involved in producing blends and to the recognisability of the source words. It appears that it is difficult to make inferences about the mechanism of blending without referring to psycholinguistic and cognitive phenomena related to the formation and processing of blends. A closer look will be taken at these in the present chapter. Section 5.1 provides an overview of studies discussing the selection of the source words that make up potential blends, and the semantic features of blends related to this selection. In section 5.2 the recognisability of the source words of blends and clipping compounds is considered in the light of psycholinguistic studies of word recognition. Section 5.3 outlines the theoretical and methodological assumptions that are utilised for the experimental study of the processing of blends and clipping compounds undertaken in the current research.

\subsection{Cognitive mechanisms of blending revealed in the form of blends}

In psycholinguistics, studies of blends were initially concerned with speech error blends as part of the research on lapsus linguae in general. Speech error blends occur, as proposed in Fromkin (1973b, p. 235), when two words seem to be able to express what the speaker has in mind, and therefore the speaker "brings them both into a buffer storage compartment, with their phonological specifications". Examples of speech error blends analysed, for example, in Fromkin (1973b) and Garrett (1975), are seen as a result of simultaneous activation of two words, when the speaker produces both competing words instead of selecting one of them. The competing words tend to be semantically related, as, for example, baggage and luggage (which are in synonymic relations). 
Production constraints that regulate intentional blends and speech error blends are considered in Berg (1998). Using the collection of blends from Pound (1967), Berg tests the predictions that: 1) "intentional blends are subject to the same basic constraints as unintentional ones"; 2) "these constraints should be less pronounced (but still be present) in wilful language patterns than in slips of the tongue [...] because speakers' intentions may reduce, but not annul, the impact of the processing principles" (Berg, 1998, p. 152). The following similarities between intentional and unintentional blends are revealed and analysed:

- the source words of both intentional and unintentional blends (Berg uses the term 'interactants') are almost always of the same syntactic category (1998, p. 152);

- if one of the source words begins with a consonant and another with a vowel, the unintentional blends tend to begin with the consonant (thus, the source word which starts with a consonant is put in the first position) and the intentional blends "follow the same trend, though to a lesser extent" (1998, p. 154);

- with regard to the length of the blend, the speech error blends are more often longer than each of their source words, but this is not as frequently the case with intentional blends (1998, p. 155);

- more than half (59.6\%) of intentional blends have an overlap (called "bridge effect" by Berg), which is also a characteristic of speech error blends (1998, p. 156);

- in terms of the semantic relationships, the source words of speech error blends are often synonyms; while this tendency is weaker in intentional blends, due to the fact that " $[\mathrm{t}]$ he volitional element in intentional blends is the rearrangement of semantic features from different lexical items so as to create a word with new meaning" (1998, p. 157).

Berg concludes that the formation of both intentional and error blends is regulated by the same production constraints, but the influence of these constraints on the form of intentional blends is weakened by the interference of the intentions of their creators. The differences in production processes are reflected in the formal differences. Berg, however, argues that the observed differences between intentional and error blends are minor in comparison with what they have in common. This agrees with the results of other studies that show that blends are subject to the same general production 
constraints that are at work in the given language. It should be noted though that the tendencies revealed by Berg apply only to a part of Pound's data because Berg intentionally excluded formations of syntagmatic origin, e.g. prinister $\leftarrow$ Prime minister which he considers to be of a different nature.

A comparative study of phonological and semantic properties of speech error blends and intentional blends by Gries (2006), discussed in Chapter 2, showed that the degree of phonological and graphical similarity between the source words of intentional blends is higher than in random word pairs, but lower than in the source words of error blends. In terms of the relative length of the source words, Gries observes the following: error blends tend to be formed of words of approximately equal lengths, while the first source word of intentional blends is often shorter than the second source word (see also similar findings in other studies discussed in Chapter 2). From the point of view of semantics, Gries' findings are compatible with Berg's assumption that the relations between the source words of error blends are more often of synonymic nature than in intentional blends. Moreover, the findings in Gries (2006) reveal that the semantic relationships between the source words of intentional blends are of a more constrained nature than between randomly selected words. The semantic relations between the source words differ for error blends and intentional blends, as also shown by Berg.

In sum, the findings of the studies above demonstrate that intentional blends and error blends are formed under the influence of similar production constraints, which, however, affect the formation of error blends and intentional blends in a different way. Studying the processing of speech error blends is not the aim of this study. Therefore, this section will further cover the research on the cognitive aspects of intentional blending.

Some recent cognitive treatments of blends are formulated within the framework of the conceptual integration theory by Fauconnier and Turner ( Turner and Fauconnier, 1995; Fauconnier and Turner, 1998, 2002). While Fauconnier and Turner use the term 'blend', their theory is not developed to deal with lexical blends as defined in this thesis. However, more recent cognitive works on lexical blends (e.g. Kemmer, 2003) have been based on conceptual integration theory, so it is useful to provide here a general outline of the theory. 
Fauconnier and Turner introduce the notion of conceptual integration, a cognitive process that operates over 'mental spaces' defined as the "small conceptual packets constructed as we think and talk, for purposes of understanding and action" (Fauconnier and Turner, 2002, p. 40). The mechanism of conceptual integration includes two 'input spaces' which are projected to a newly created space - the 'blend', and this blended mental space "inherits partial structure from the input spaces, and has emergent structure of its own" (Fauconnier and Turner, 1998, p. 1). The cognitive spaces discussed by Facounnier and Turner may include a range of elements such as the roles of the speaker and the listener, time, space, and various characteristic features of a denotatum (for instance, questions and answers are elements of the cognitive space 'debate'). As a result of conceptual integration, or conceptual blending, as the authors prefer to call it (Fauconnier and Turner, 1998, p. 3), various connections between elements of the input spaces are exploited and brought into the blended space. Some of the connections that may be activated are similar speaker roles in two input spaces, similar characteristic features of the two denotata, associative and metaphorical connections.

The main sphere of use of conceptual blending theory for analysing language or speech is at the textual level, or as method of discourse analysis. It is possible though, as is postulated by its authors, to apply it to the analysis of noun-noun compounds. For example, in a compound land yacht ('a luxurious car'), the semantic characteristic 'expensive' which is associated with yacht is brought to the blended space of the compound meaning.

Some linguists extend the field of application of this theoretical framework to the analysis of lexical blends, in which both the form and the meaning of the source words are integrated. A prime example is Kemmer (2003). First of all, Kemmer confirms that the analysis of blends as a morphological phenomenon must not involve attempts to divide them into traditional morphemes or morpheme-like parts, and therefore suggests a schema-based approach (which she concedes is not rule-based but constraint-based), grounded on the principles of cognitive grammar. Her study is focussed on intentional blends.

Following Langacker (1987, 2000) and MacWhinney (2000), Kemmer (2003, p. 78) defines schemas as "generalizations extracted from linguistic forms and meanings", and cognitive representations as "consisting of perceived similarities across many instances 88 
of usage". Linguistic schemas work on different levels of language, for example, on the phonological level a schema would be a specific phonotactic pattern, or a repeated string of phonemes such as /str/ for words like strength and strip (2003, p. 78).

The following characteristics of lexical blends (not necessarily conditions of defining them but rather tendencies that are observed in instances of blending) are given in Kemmer (2003, pp. 75-77):

- blends combine parts of 'lexical source words' (term used by Kemmer), and this distinguishes them from compounds;

- morphological structure is not particularly relevant to blends because blends are "not really composed of morphemes in the sense of recurrent minimal meaningful parts" (2003, p. 77);

- phonological properties are, on the contrary, highly relevant to blending; this is closely connected to the fact that, instead of morphemes, blends are composed "of phonological strings that trigger meanings" (2003, p. 77).

Kemmer's conclusions concerning the formation of blends and their lack of morphological analysability are compatible with similar observations in other studies on blends (e.g. Bauer (1983) and Cannon (1986), see also Chapter 2). According to Kemmer (2003, p. 93, ff.), the last characteristic listed above, i.e. the notion that there are "phonological strings that trigger meanings", concerns the association between sound and meaning that is realised in the words in general. Some examples of this are phonaesthemes and clippings. They, alongside blends, are instances of a more general phenomenon of association between sound and meaning which does not necessarily take place at a word or morpheme level.

Regarding lexical blends, Kemmer reiterates, on the one hand, that phonological patterns play a crucial role in the formation of blends. On the other hand, she claims that blends involve conceptual integration of meaning, that is, the meaning of the blend includes certain (but not all) elements of the meanings of the source words. Thus, the meanings of the source words are associated in this interpretation with the 'input spaces' of Fauconnier and Turner. The meaning of the blend may also include some emergent structures that may not have been present in either of the initial mental spaces. Altogether this meaning represents the 'blended space' (as Fauconnier and Turner term it) that emerges as a result of conceptual integration. Thus, "[t]he semantics 
of a lexical blend is a coherent cognitive structure that selectively incorporates and integrates aspects of the semantics of the activated words" (Kemmer, 2003, p. 71). Kemmer also underlines (2003, p. 83) that the degree of conceptual integration in the case of blends is higher than in the case of compounds. Similarly to the compound constituents, the constituents of established blends can gradually lose their associations with their sources, i.e. the source words of blends, and also may undergo partial meaning loss. For example, blends like glitterati, Briterati, chatterati, etc., are picked by Kemmer to illustrate the observation that blending can give start to a productive process the result of which will be a lexical family and, eventually, a bound morph. The common splinter in this case is perceived as a phonological schema repeated in different lexemes. To this one can add that the relation to the original source word literati can be weakened or eventually lost in such a lexical family, and therefore it will no longer be possible to talk about the conceptual integration based on the words literati and glitter, etc., or about the recognisability of the word literati in glitterati or chatterati. Such weakening of the semantic content of splinters as a result of frequent usage is compatible with the effects of the weakening of the literal meaning in multi-word units such as going to, discussed, for example, in Bybee (2006). Kemmer observes that splinters which are used in more than one blend may undergo partial meaning loss. A similar tendency is discussed in Lehrer (1998): in blends such as shopaholic, workaholic etc. the splinter -(a)holic has lost the semantic connection with the word it originated from, i.e. alcoholic and acquired a specific meaning of its own: 'addicted to something'.

The reasons for such changes can be explained in the framework of Exemplar Theory (e.g. Goldberg, 1995; Pierrehumbert, 2001; Bybee, 2006). According to exemplar models of language use, individual memories of linguistic phenomena such as sounds, words or multi-word combinations (i.e. exemplars) are stored in the memory of language users. The remembered exemplars represent a range of manifestations or, for example, sound or meaning, and further exposure to similar phenomena can alter the stored representations. In particular, new tokens which are similar to the remembered tokens in respect to a particular feature can result in strengthening of the representation of this feature. On the other hand, multiple new tokens which are different from the remembered tokens can cause a change in the stored representation, reflecting the observed differences. The changes in the meaning of productive splinters such as -(a)holic or -(a)thon, therefore, may be the result of their frequent use in words which 
do not have certain semantic components present in the source words of the splinters (e.g. relatedness to alcohol, or a running distance).

Returning to the material of this research, I must note here that the strength of the semantic link between blends and their source words has to be taken into account if we make comparisons between different structural types of blends (and also between blends and clipping compounds) in terms of their potential to be decomposed into source words. The principles of selecting lexical data for this study (that is, concentrating on novel blends, see section 4.1) can help filter out cases where the semantic link between the source word and the splinter has been weakened or lost. Controlling this factor to the extent possible makes it easier to study the influence of other factors, in the present case that of the structural type.

\subsection{What factors determine recognisability?}

Word recognition is studied in psycholinguistics in relation to the mental lexicon, which, as described in Marslen-Wilson et al. (1994), stores "the listener's mental representation of what words sound like and what they mean". Extensive research on mental lexicon, e.g. Badecker (2001, 2007), Moore et al. (2009), has presented evidence that the mental lexicon stores various information not only about the sound and meaning of words, but also about morphological structure, collocations, relations between compound constituents, etc. The information stored in the mental lexicon is, on the one hand, enormously diverse and, on the other hand, structured in multiple ways that allow relatively easy access to its various bits. Models of the mental lexicon (see, for example, Aitchison (2002) for a summary) reflect two essential features assigned to the lexicon by different researchers to a greater or lesser extent: 1) the lexicon stores whole lexemes; 2) the lexicon stores sub-lexical constituents together with the rules or schemas according to which lexemes are to be constructed online when needed. Whether rules or representations (or both) are more characteristic of the mental lexicon has been the subject of debate in psycholinguistics since at least the 1970s (see Pinker (1999) for an overview). According to the first approach which is often called the word-based approach, the key feature of the lexicon is the storage of full lexemes. This approach is maintained, for example, in Bybee (1995) and Blevins (2003), and has received extensive support from experimental studies, such as Bertram et al. (2000) and Baayen et al. (2002). On the other hand, supporters of the decompositional approach, e.g. Taft 
and Forster (1975), Halle and Marantz (1993), assume that morphologically complex words have morphologically complex representations in the mental lexicon (see, for example, Taft (2004) for experimental evidence).

Extensive experimental evidence for the storage and retrieval of words has been derived using the priming technique. In priming experiments, the response of participants to a stimulus referred to as the 'target' or 'probe' (auditory or visual, word or nonword, etc.) is studied in relation to another stimulus presented before the target, the 'prime'. The relatedness of prime to target (that is, whether the prime is identical to the target, phonologically or graphically, morphologically or semantically related to it, or unrelated) is manipulated in order to detect whether the primes which are related to targets in a particular way enhance or inhibit the participants' reaction to targets (Neely, 1991; Marslen-Wilson et al., 1994). The prime can either be presented overtly (that is, so that the participants in an experiment are consciously aware of the prime), or, according to the 'masked priming paradigm' developed by Forster and Davis (1984), presented only for a fraction of a second, with no intervening items between the prime and the target stimulus presentation. In the case of masked priming, most of the participants fail to consciously notice the prime stimulus. On the assumption that recognition starts before the results of this process are registered by consciousness, the effects of masked primes on the facilitation or inhibition of target stimuli indicate quite early stages of word recognition.

Among the variety of tasks used in priming experiments perhaps the most widely used are the lexical decision task and the naming task. In a lexical decision task, participants have to answer whether the target stimulus is a word or not (usually by pressing either a 'yes' or a 'no' button). In a naming task, participants have to produce a word, sentence or another piece of lexical information as a response to a stimulus (word, picture, etc.). Naming tasks are often used in studies of word production, while lexical decision tasks are used in studies of word recognition.

Growing evidence from psycholinguistic studies (in particular, from priming experiments) suggests that both word storage and morphological (de)composition is found in the mental lexicon, and that there are factors which influence whether, during the process of word retrieval, the full form is more easily accessible than its morphological constituents or vice versa. For example, according to Marslen-Wilson et 
al. (1994), not all morphologically complex forms are represented in the lexicon in the same manner (in particular, they highlight differences between semantically transparent and semantically opaque complex forms). The factors which seem the most plausible candidates for explaining the choice between full word storage and online construction in each particular case are the frequency of the word and the productivity of the morphological pattern. Concerning the productivity of the morphological pattern, Bauer (2001, p. 122) notes that evidence from experiments on word processing, and also studies on language acquisition suggest that "some morphological processes are stored in the brain independently of the words in which they occur". An explanation of this in the framework of probabilistic approach to linguistic phenomena is provided in Hay and Baayen (2005).

Research on the representation of morphologically complex words has been conducted on material of various morphological categories, but the studies of the representation of compounds are of particular interest in the context of the present research for two reasons. Firstly, as expressed by Libben (2006, p. 2), compounding can be "considered to be the universally fundamental word formation process" in the sense that in most world languages compounding is a productive type of word formation. This claim is supported, for example, by Štekauer et al. (2012), who recorded compounding in 50 (90.91\%) of the sample of 55 languages used for a typological study of word formation. Therefore, the insights from studies of compounds are likely to be generalisable to all morphological processes. Secondly, as blending is similar to compounding in many aspects and can be regarded as a subtype of compounding (see section 3.1. for detailed argumentation), it is reasonable to assume that the processing and understanding of blends will be similar to those of compounds. Moreover, given that experimental studies of blends are rather scarce, the research on compounds (which is, on the contrary, quite extensive) can be especially useful.

An overview of the findings in psycholinguistic studies of the representation and processing of compounds is given in Jarema (2006). The following effects on compound representation and processing are reported:

- semantic: semantically related primes have been reported to influence the processing of semantically transparent (Sandra, 1990), or even of semantically opaque (Libben et al., 2003) compounds; 
- morphological family effects: the morphological family size of constituents, i.e. the number of words that contain morphemes that are also the constituent parts of a compound has an effect on the accessibility of each constituent in a compound;

- position effects: experimental results testify a "significantly greater magnitude of priming of first constituents as compared to second constituents", i.e. that the first elements of compounds are more easily primed than the second ones (Jarema, 2006, p. 54);

- headedness effects which interact with position effects: the priming of the head element in compounds depends on the semantic transparency of this element and also on whether the right-hand or left-hand element is the semantic head (Jarema, 2006, p. 56).

As summarised in Libben (2006, p. 6), the way in which compounds are represented in the mental lexicon can be modelled differently, depending on whether computational efficiency or storage efficiency is assumed to have higher priority in the organisation of the mental lexicon. Three types of models are distinguished by Libben, as outlined in (5.1):

a) According to the models assuming maximization of computational efficiency, compounds are represented in the lexicon as full forms independently of the representation of their constituents, to allow immediate access to the full form, without reconstructing it from the constituents.

b) According to the models assuming maximization of storage efficiency, only the constituents are represented, and the full form is constructed online from the constituents, instead of being accessed directly.

c) According to the models assuming maximization of computational and storage opportunity, both compound constituents and full compounds are represented, and their representations are linked in the lexicon.

Experimental studies reported in Libben and Jarema (2006), e.g. Myers (2006), Semenza and Mondini (2006), provide evidence for (5.1c). In sum, the findings of the research on the representation and processing of morphologically complex words (including compounds) suggest that in each particular case of word recognition the mechanism of 
accessing mental representation depends on the various properties of the words that are processed. In relation to word frequency, for example, this can be illustrated by the differences in processing low frequency words and high frequency words. As summarised in (Libben, 2006, p. 9), "novel words will only be processed in terms of their constituent morphemes because there is no whole-word representation to activate". Known words which can have whole-words representations "may show graded trade-offs between whole-word and constituent activation". In this respect, high frequency words differ from lower frequency words: "For very frequent words, wholeword activation would be expected to be both stronger and faster. For less frequent words, the morphological route might, in fact, 'get there first'”.

On the one hand, this is applicable to blends as well as to compounds because blend words can be accessed both as a whole and through the mental representations of their source words. The former case especially concerns well-known blends such as brunch or motel which not only have long been functioning in the language, but may have started to lose the semantic connection to their source words. Novel blends, such as those collected for the present study, are more likely to be processed through access to their source words. On the other hand, the representation of blends should be viewed as different from the representation of compounds because of the formal differences between these morphological categories. To be more precise, the fact that blends only partly retain the material of their source words can result in the failure to access the representations of the source words. The following chapters explore how and to what degree the forms of blends and, in particular, the degree of preservation of their source words, influences the processing of these words.

As already stated above, the experimental studies dealing with blends are scarce. One exception is a series of experimental studies on intentional lexical blends presented by Lehrer (Lehrer, 1996, 1998, 2003, 2007; Lehrer and Veres, 2010). The aim of the earliest of these studies was to identify the factors that influence recognisability of the blends' constituents, i.e. the original source words which had formed blends. The hypothesis suggested in Lehrer (1996, pp. 360-361) is as follows: “[...] the factors that lead to the successful identification of the words that make up the blends (the targets) and their interpretation are sensitive to the same factors that have been found relevant in psycholinguistic studies of lexical access: frequency, neighborhood density, and semantic priming". 
From a common sense point of view, this hypothesis seems very likely to be confirmed because lexical access to blends or their parts should work according to the same mechanisms as lexical access in general, unless there are strong reasons for blends to be different from all other lexemes in this respect.

Lehrer's definition of blends is related to her understanding of compounds, the principle difference between compounds and blends being that "in compounding, complete morphemes are present, whereas in blending, one or both parts are clipped" (1996, p. 360). Another important assumption made by Lehrer is that the constituent parts of blends may include the following: 1) clippings (which means that Lehrer's approach does not allow for an etymological distinction between blends and clipping compounds); 2) splinters, defined as "parts of words in blends which are intended to be recognized as belonging to a target word, but which are not independent formatives"; 3) combining forms, i.e. either neoclassical combining forms such as electro, or semantically independent bound morphemes such as -scape, -fare (1996, p. 361). Lehrer approaches the three types of blend constituents diachronically: it is postulated that splinters from blends can with the course of time become combining forms, and that both combining forms and splinters can eventually (though not always) become clippings or affixes (cf. Kemmer's diachronic analysis of blend constituents discussed in section 5.1).

The experiment reported in Lehrer (1996) did not involve time pressure. The participants were shown 72 blend words and were asked to identify their source words and to provide glosses for them. Some of the participants had to read blend words in isolation, some in a sentence context. As a result of this experiment, the initial hypothesis was confirmed, and it was claimed that the mechanisms that are involved in identifying and interpreting blends "are the same as those for the lexical retrieval of any other words" (1996, p. 385). Strictly speaking, given that this study included an off-line task only, its results cannot be used for evaluating automatic processes of lexical retrieval. Therefore, Lehrer's claim above can be referred to the processing of blends in a more general sense, rather than their retrieval. Nevertheless, these results allowed Lehrer to come to a conclusion that "blends are not so exotic after all" (1996, p. 385). This is in agreement with more recent studies that aim to investigate the characteristic patterns of the formation and processing of blends (see below). In addition, it was concluded that the source words are identified more easily if blends are presented in context. This finding is in line with earlier findings concerning the role of context in 
understanding new words (see, for example, Baayen and Neijt (1997) for the discussion of contextual anchoring of derivatives, and Bayer and Renouf (2000) for a study of the role of context in understanding compounds). Such a result suggests that contextual clues may be used to reconstruct the full form of the source words of blends, in addition to the graphical or phonological material that is preserved in the blends.

A later experiment reported in Lehrer (2003) targeted the recognisability of the source words within blends, and introduced time pressure. During the experiment, a blend appeared on the screen, participants were asked to press a YES button if they were able to identify the two source words of the blend and then to pronounce these words into the microphone. If they failed to identify the source words, then after 20 seconds the blend word disappeared. A subsequent task was used to determine which blends were not known to the participants. That is, after the experimental task, participants were provided with a list of all the blend words from the task, and were asked to circle those words which they had never seen before the experiment. Reaction times and accuracy rates (i.e., whether the source words were identified correctly or incorrectly) were compared across four types of blends:

a) word + splinter (WD, a subtype of AD in the notation adopted in this thesis);

b) splinter + word (AW);

c) two splinters (AD);

d) complete overlap (WW).

The results of the experiment show that the source words of blends consisting of two splinters (c) were less often named correctly than the source words of other blends. However, the differences between the four blend types in terms of accuracy rates were not statistically significant. Nevertheless, the observed tendency suggested that the source words of AD blends were more difficult to identify than the source words of blends from other groups.

Semantic relationships between blends and their constituents are studied in Lehrer (1998). Lehrer examines compound-like neologisms that result from blending, focussing on the dynamic process in which a splinter becomes a productive bound morpheme, i.e. a combining form which is neither a root nor an affix and which resembles neoclassical bound morphemes in its morphological properties. The results of the experiments 
reported in Lehrer (1998) demonstrated that the coinages that contain combining forms that were initially parts of blends and which subsequently became productive are perceived as hyponyms to the blends with those combining forms, rather than their cohyponyms. For example, Cinerama can be explained as a kind of panorama, and catnap as a kind of kidnap. This is contrary to the claim in Warren (1990, p. 123) that forming new words with final combining forms results in producing co-hyponyms of the original formations with those combining forms, not their hyponyms (e.g. spendaholic is not a type of alcoholic but a type of addict). The findings in Lehrer (1998) suggest that there is (at least temporarily) a semantic link between the blend as a whole and its splinters. Comparing such productive splinters with the neoclassical combining forms they resemble, Lehrer observes certain differences between them. Firstly, while most neoclassical combining forms are not associated (at least in contemporary English) with specific source words, the "productive splinters from blends retain a connection to their source words" (Lehrer, 1998, p. 16). As Lehrer (1998, p. 16) further admits, the semantic connection between the productive splinters and the words they originate from may be lost over time "if for some reason the source word were to become archaic or obsolete". I would add to this that the weakening or complete loss of this semantic link may not necessarily happen only if the source words become archaic. As exemplar models (see above) suggest, frequent use as a combining form (i.e. without direct association with a particular source word) may be sufficient for such a semantic change. This would explain the perception of new formations with well-established combining forms as cohyponyms, observed in Warren (1990). Another difference between splinters and neoclassical combining forms is that "[s]plinters, unlike neoclassical compounding forms, may reflect radical resegmentation of words, quite different from expected segmentation" (Lehrer, 1998, p. 16). However, from this statement it is not clear what kind of segmentation should be expected.

Evidence of automatic and rapid decomposition of blends was sought in an experiment with a lexical decision task described in Lehrer (2003, also reported in 2007). Participants in the experiment saw blend words (e.g. fruitopia) presented as masked primes for $100 \mathrm{~ms}$ before target words (e.g. FRUIT). They were required to decide, as quickly and accurately as possible, whether or not the targets were real English words. The experiment involved three conditions, each using masked priming, with different participant groups in each condition: 1) the masked prime was a blend and the target word was one of the source words (e.g. fruitopia-FRUIT); 2) the masked prime was 98 
identical to the target (e.g. fruit-FRUIT); 3) the masked prime, matched in length with the blend prime was orthographically and semantically not related to the target (e.g. stillborn-FRUIT). The main hypothesis was that blends would facilitate the recognition of their source words. In particular, it was hypothesised that the strongest priming effect would be caused by presenting identical primes, unrelated primes would have the least effect on the recognition of target words, and that blend primes would facilitate the recognition of target words, but not as successfully as identical primes. The facilitating effect of blend primes would, thus, be the evidence of rapid automatic decomposition of the blend (Lehrer, 2003, p. 378). In this task, target words (e.g. FRUIT) were recognised faster in the condition where they were preceded by identical primes (e.g. fruit) than in both other conditions. However, the difference between the conditions with blend primes and with unrelated primes was very small $(7 \mathrm{~ms})$ i.e. there was no significant facilitation of the recognition of the source word by blend primes. The results reported may seem unreliable because "the order was as predicted, but an analysis of variance showed that the time differences were not significant at the .05 level" (Lehrer, 2007, p. 128). This means that no evidence of automatic decomposition of blends into their source words in the process of word recognition was found in the experiment, either because no automatic decomposition of blends really takes place, or because it has to be elicited using different methods from those in Lehrer's experiment. It may also be the case that the decomposition of blends takes place during later stages of processing and therefore cannot be detected using the masked priming technique.

Other experiments reported in Lehrer $(2003,2007)$ demonstrated evidence of associations between blends and their source words in language users' memories. In an identification and production task, the participants were shown a blend on the screen and were asked to press a foot pedal as soon as they identified the constituent words, and then to pronounce the constituent words. Following this, the same participants were asked to complete a 3-letter sequence so that it made an English word. It was found that those who saw a blend (e.g. dramedy) were twice as likely to produce a blend source word (e.g. comedy) as their completion of the letter sequence (e.g. COM) as the respondents who did not see the blend. Such an effect can be the result of pre-activating the source words of the blend in the identification and production task, so that their representations remain active during the stem completion task. However, one cannot be sure that the effect is not due simply to graphical overlap regardless of whether or not 
the overlap is linked to blend structure, as the experiment did control for simple graphical overlap.

As predicted by the researchers, participants in the identification and production task were more likely to name both source words of a blend correctly in the case of fully overlapping blends, and blends consisting of two splinters turned out to be the most difficult to decipher. However, the difference in the percentages of correct responses for these groups of stimuli was not found to be significant. As discussed in Lehrer (2003: 376), one of the possible reasons could be that the lists of blends used for the task 'were not matched for difficulty'. In addition to this, there could be other factors at play, such as different degrees of lexicalisation of the blend stimuli, or different degrees of productivity of the splinters (some blends in Lehrer's tasks contained splinters that had already lost their connection to the source words and became affixes, e.g. -licious).

Lehrer (2003) makes an important pragmatic observation concerning blends. She notes that some of the creative blends are unlikely to have been created in order to facilitate communication, despite their compact and handy form. Rather, they create additional difficulties in understanding due to the opacity of their meaning (this is regarded not as the result of lexicalisation process, but as an immanent quality of the creative blends from the moment of their coinage). Thus, the author concludes that the users of such novel blends must have the perlocutory intention "to catch the hearer's attention" (2003, p. 370), to make the neologisms memorable. It is worth mentioning that this is not the only observation of this 'attention-catching' property of creative blends, a property that may be seen as distinguishing blends from other types of coinages (see, for example, Renner (2006) and Fandrych (2008a) for similar claims).

As was later summarised in Lehrer (2007), where the experiments above were reviewed in the light of more recent findings and theoretical insights, the following factors were shown "to contribute to the identification of the source words making up the blend and to facilitate an interpretation" (Lehrer, 2007, p. 126):

- context;

- the number and percentage of letters (or phonemes) of the source word present in the splinter;

- the frequency of the source words of the splinter; 
- the number of graphical 'neighbours' of the source words (i.e. words that differ from the targets by one letter or phoneme);

- the semantics of the blend, more precisely, the semantic link between the source words.

Some clues to the mechanisms of blend formation can be drawn from recent experiments on eliciting blends. Two examples are experiments during which the participants were asked to name non-existing hybrid objects shown in pictures. The experiments were carried out in German and Hungarian (Borgwaldt and Benczes, 2011) and, later, in Ukrainian (Borgwaldt et al., 2012). The semantics of the blends created in the course of the experiments was restricted by the underlying semantics of the input stimuli. The same is true for the experimentally elicited blends in German and Hungarian. Thus, two types of hybrid objects were displayed in the experiments: 1) hybrids of two identifiable objects such as plants or animals, e.g. a hybrid of a chicken and a fox; 2) objects having a salient shape, such as a clock in the shape of a flower. The first type of objects can therefore be named by a coordinative compound like fox-chicken or chicken-fox, and the compounds that can be produced as names for the second type of objects are more likely to be endocentric, such as flower clock (a kind of clock).

The majority of the hybrid names produced by both German-speaking and Hungarianspeaking participants of the first study were noun-noun compounds, and only $5 \%$ of responses for both groups were blends. In contrast to this, in an experiment with Ukrainian participants using the same picture stimuli, 55\% of the hybrid names were classified as blends and clipping compounds (out of which $10 \%$ were clipping compounds). Analysing the Ukrainian data, Borgwaldt et al. (2012) investigate the structure of the blends and compare their features to the ones in other corpora of lexical blends. In the Ukrainian data, the first source word of blends was on average longer than the second one, which had been observed before as a characteristic of speech error blends, but not intentional blends (Borgwaldt et al., 2012, p. 90). Some structural features of blends produced by Ukrainian speakers seem to differ from what is described in Bat-El (2006), Gries (2012) and other publications, in terms of the relative length of the source words and the contribution of the second source word to the body of the blend. These differences may be either a feature of Ukrainian blends in general, or only of the spoken blends induced using a particular experimental technique. 
English blends coined under experimental conditions are analysed in Arndt-Lappe and Plag (2013). In their study, English-speaking participants were provided with a list of 60 pairs of words, and were asked to produce a blend on the basis of each pair. The word pairs were made of words which could potentially be interpreted as coordinative compounds, e.g. bar + restaurant (Arndt-Lappe and Plag, 2013, p. 543), with the assumption that blends are typically made of words in coordinative relationships. The findings concern the overall structure of the experimentally induced blends, the position of the switch point and the position of the main stress in blends in relation to one in the source words.

In terms of the general structure, Arndt-Lappe and Plag (2013) observe that 24\% of blends preserve at least one of their source words in full, the remaining being AD blends in the notation used in this thesis. Out of those which retain all the material of at least one source word, two-thirds (that is, about $16 \%$ of all data) preserve W2 in full, that is, they are AW blends. Arndt-Lappe and Plag (2013, p. 546) note that this reflects the tendency of blends to retain more material from W2 than from W1 (cf. 55.7\% of blends preserving W2 in full in Gries (2004, p. 664) and 16.2\% of AW plus 5.7\% of WW in my collection discussed in Chapter 4).

Arndt-Lappe and Plag (2013) argue that the position of the switch point and the position of the main stress in blends are closely related because in their data the switch point tends to be placed on the main-stressed syllable of W2. In section 4.3 this tendency is observed for AD blends, but not for the other structural types in the present collection.

The studies described in Borgwaldt et al. (2012) and Arndt-Lappe and Plag (2013) involved experiments in which respondents were either explicitly or implicitly asked to create blends. The data from these experiments allow inferences to be made about the factors which influence the formation of blends in language. However, it is not known to what extent the factors regulating the formation of experimentally induced blends reflect the formation of blends outside the experimental conditions. Another way to look at the same issue is to study the comprehension and evaluation of existing blends by language users, which is done in this research.

\subsection{Methodological prerequisites for an experimental study of blends}

As was discussed in Chapter 4, the switch point in blends is related to the uniqueness point, 'a point in the word where it no longer overlaps with other words in the initial 
cohort' (Warren, 2013, p. 129). In Gries (2006), a theoretical construct of 'selection point' is used for the analysis of recognisability of the source words and clipping compounds. The selection point in Gries' sense is not the same as the uniqueness point, although the two notions are related. The selection point is the point after which the word is not necessarily the only one starting with a certain letter / phoneme string, but the most frequent one. In order to identify the position of the selection point for the first source words of blends (W1), Gries extracted all the tokens in the CELEX database which start with a given string of letters / phonemes. For the second source word (W2) of blends (not of clipping compounds), word endings were looked up in the CELEX database, and the graphemes or phonemes necessary for recognising W2 were counted from left to right, accordingly.

However, experiments with rhyming word pairs (Marslen-Wilson and Zwitserlood, 1989) have shown that it is the initial portion of the word for which recognisability can be thus assessed, not any part of the word. This does not necessarily mean words cannot be recognised by their endings. It is possible that the recognition of a word by its ending does not work in the same way as by its beginning, especially if we bear in mind that the recognition of words by their beginnings is a consequence of how the words are heard in naturally occurring speech. According to the Cohort model of word recognition (Marslen-Wilson and Welsh, 1978; Marslen-Wilson, 1987), when a word is heard, several words with the same beginnings are contacted in parallel, and this set of words forms the 'word-initial cohort', but this does not work in the same way with word endings or other parts of words.

Thus, although one can compare the recognisability of W1 in blends and clipping compounds using the notion of uniqueness point, the situation becomes different when it comes to $\mathrm{W} 2$ recognition. On the one hand, $\mathrm{W} 2$ of clipping compounds seems to be in a privileged position in comparison with $\mathrm{AD}$ blends because the beginning of $\mathrm{W} 2$ is retained in clipping compounds, and this can be the reason why it may be sufficient to retain a smaller portion of W2 in clipping compounds than in blends. On the other hand, the recognition of W2 can be hindered in clipping compounds because: 1) the W2 splinter is positioned not at the beginning of AC and therefore may not be perceived as the beginning of a word; 2) the W2 splinter of AC (as well as W1 splinter, according to Gries) may be too short to reach the recognition point. As for $\mathrm{AD}$, because the beginning of W2 is not available for recognition, it is not possible to talk about the recognisability 
of W2 in $\mathrm{AD}$ using the left-to-right notion of recognition point in the sense of MarslenWilson's Cohort model.

For these reasons, it is important to study the recognition of both W1 and W2 in blends and clipping compounds using an experimental paradigm, rather than relying only on corpus findings. As was noted in section 4.5 , some of the conclusions regarding the differences between $\mathrm{AC}$ formations and blends require further evidence because they are founded on scarce data. The corpus of novel blends and clipping compounds that was collected for this research is not balanced, and contains only 23 formations that can be classified as clipping compounds, because such formations are extremely rare in the sources that were used for lexical data collection (and perhaps reflect the general tendency in English). The unequal numbers of lexemes of different structural types and, in particular, the low numbers of AC forms, constrained the statistical power of the analysis in Chapter 4. However, even if the generalisations concerning the phonological, structural and semantic properties of clipping compounds had to be formulated using a small sample, it is possible to get a sufficient number of observations regarding the recognition of their source words from human participants in an experimental study.

The following two chapters of this thesis present the results of two experiments addressing the recognisability of the source words of blends and clipping compounds. The first (Chapter 6) is a web-based survey studying the evaluation of the definitions of blends and clipping compounds and the influence of the source words on it. The second (Chapter 7) is a psycholinguistic experiment focussing on the recognition of the source words of blends and clipping compounds. I must note here that I do not aim to find evidence of automatic decomposition of blends into source words during word recognition (as was the aim of one of Lehrer's experiments), nor do I attempt to determine at which stage of word processing such decomposition may happen. Before such questions can be addressed, it is necessary to determine whether such decomposition takes place at all. In addition, the aim of the experimental study is to check whether both blends and clipping compounds are decomposed equally successfully, and whether any differences in this respect can be observed for blends with different types of structure. 


\section{Chapter 6. What can be predicted from the way predictionary and other blends are defined? A web-based study.}

The analysis of corpus data in Chapter 4 has contributed to earlier findings concerning the phonology, structure and semantics of blends, and has revealed some structural differences between blends and clipping compounds. The next objective of this research is to investigate how the phonological and orthographic material from the source words that is retained in the blends affects the way language users understand the blends. This implies finding out whether blends and clipping compounds are formed in such a way that language users can recover the meanings of their full constituents. I have attempted to achieve this goal by looking at readers' evaluation of alternative definitions of the blended words provided in a web-based survey.

\subsection{Objectives and hypotheses of the study}

As discussed in sections 4.2-4.4, clipping compounds such as finlit are more likely to have been formed as contractions of (relatively) frequently used collocations, e.g. financial literacy, than blends formed by fusing together the beginning of one word with the end of another, e.g. collabulary $\leftarrow$ collaborative + vocabulary. Where blends are concerned, I have found no reasons to believe that shortening is the primary mechanism underlying their formation. On the contrary, the data from the research on blends support the assumption that preserving enough material from the source words for their full form to be recognisable from the blend is a crucial factor of blend formation. The findings from studies comparing speech error blends and intentional blends (as summarised in Chapter 5) demonstrate that in intentional blends, unlike in error blends, the shorter source word usually provides the initial splinter, and the final splinter tends to come from the longer source word. One possible explanation is that in a blend, when the beginning of a word has to be lost, the tendency is to preserve as much material of this word as possible, in order to enhance recognition (at least as much as is possible while also shortening the whole formation in order for it to appear a single word).

Clipping compounds differ from blends not only in terms of formal structure, but also because they preserve relatively smaller portion of each of their source words, as is claimed in Gries (2006), and further discussed in Chapter 4. As suggested above, clipping compounds are instances of shortening, and therefore the compactness of their form can be prioritised over the recognisability of their source words. If this is the case, the readers or hearers of clipping compounds will have more difficulty reconstructing their 
source words than the source words of blends. That is, to understand the meaning of the AD blend collabulary, a reader / hearer will not need to be exposed to the words collaborative and vocabulary, while to understand what the clipping compound (AC) finlit means one may need to know that it stands for financial literacy. What could be considered as evidence in favour of this assumption? Meanings of words are reflected in their definitions. An indirect way to estimate understanding of blends and clipping compounds is, therefore, to study the response of readers / hearers to their definitions. If a form with low recognisability leads to difficulty in the retrieval of its source words, then readers / hearers may need an overt explanation of what the formation stands for, that is, the source words should be present in the definition. The survey presented in this chapter aims to investigate whether the presence of the source words in the definitions of blends and clipping compounds influences the responses of readers to those definitions.

If no systematic differences in responses to the definitions of blends and clipping compounds can be found, irrespective of whether the source words are present in the definitions or not, this would suggest the above assumption is not true. Such a finding could then mean that the source words of clipping compounds can be recognised as easily as the source words of blends (or as W1 of blends) because the word onsets are preserved both from W1 and W2 of clipping compounds. Hence there will be no significant difference between responses to the definitions of blend words and of clipping compounds, at least in respect of the presence of W1. Alternatively, such a result might mean that shortening is the primary mechanism underlying the formation of both blends and clipping compounds, which would cause no systematic difference in the recognisability of the source words.

I propose to use readers' evaluation of the definitions of existing blends and clipping compounds as an indirect measure that might reflect some aspects of how these words are processed. Assuming that full preservation of source words results in a higher degree of transparency than partial preservation, it is reasonable to compare the structural types exemplified in 6.1. The use of structural types here is according to the same principles as in Chapter 4, the only difference being that it was decided to distinguish between WD and AD ( $b$ and $d$ in 6.1) to check whether full versus partial preservation of $\mathrm{W} 1$ is important. 
a) blends preserving both source words (WW), as in predictionary $\leftarrow$ prediction + dictionary;

b) blends preserving the first source word in full (WD), as in jazzerina $\leftarrow$ jazz + (ball)erina;

c) blends preserving the second source word in full (AW), as in voluntourism $\leftarrow$ volunt(eer) + tourism;

d) blends preserving only parts of both the source words (AD), as in blizzaster $\leftarrow$ blizz(ard) + (dis)aster;

e) clipping compounds, consisting of the beginnings of two source words (AC), e.g. scigov $\leftarrow$ sci(ence $)+$ gov (ernment).

Because of the low degree of formal transparency of clipping compounds, the easiest and most efficient way to define, for example, foco would be to provide its full counterpart, i.e. food court. On the other hand, using the words collaborative and vocabulary may be not so crucial for defining the blend collabulary which has a high degree of transparency (see Chapter 4 for the discussion of the degrees of transparency of different types of blends and clipping compounds).

The data for this study come from a web-based survey inviting native speakers of English to read a number of sentences containing novel blends or clipping compounds (selected from the corpus described in Chapter 4) and to evaluate definitions provided for these blends or clipping compounds. The experimental stimuli were presented visually to the participants, and so for each of the target words the decision about inclusion or exclusion of material from the source words (marked by parentheses in 6.1) is based on orthography.

The following hypotheses are tested in the experiment:

1. Participants' evaluation of the definition of a target word will be higher for more transparent target words (a-c above) than for less transparent ones (d-e).

2. The definitions of target words of different structural types will be evaluated differently depending on the type of the definition. Specifically, if a target word retains only a fraction of one $(A W, W D)$ or both $(A D, A C)$ its source words, then 
the definitions which contain these source words will be given a significantly higher evaluation. No such difference will be observed for definitions of blends which fully retain both source words (WW).

\subsection{Data and methods}

\subsubsection{Participants}

Native speakers of English, aged over 18, were invited to participate in the web-based survey via e-mail lists and online announcements containing the link to the survey webpage (see Appendix 3). No restrictions were placed on which particular variety of English the participants should speak. Even though the information about the survey was circulated mainly among students and staff at the Victoria University of Wellington (New Zealand), there was a possibility that residents of other English-speaking countries took part in the survey. Likewise, the stimuli came from various sources (as they were selected from the collection discussed in Chapter 4, see 6.2.2) and originated from different varieties of English. Because of this, no a priori decisions about the spelling of the stimuli were made, and the spelling of the original sources was preserved (see section 6.2.3, and also the full set of stimuli and instructions in Appendix 4). The participants entered a prize draw as a reward for taking part in the survey (full instructions for the participants are provided in Appendices 3 and 4). Participation was anonymous, but the survey included questions about the respondents' age group, sex, first language, and whether or not they had taken a university course in linguistics. The full list of survey questions is provided in Appendix 4.

Responses from 117 people were received, but 5 were excluded from the analysis because 1) the first language of 2 respondents was not English, 2) 3 respondents gave answers to only a small fraction of the survey.

\subsubsection{Stimuli}

The set of experimental data consisted of 79 lexemes of the following structural types exemplified in Table 8. The blends were selected in such a way that groups of AC, AD, $\mathrm{AW}, \mathrm{WD}$ and $\mathrm{WW}$ forms were of approximately equal size (one of the AC forms - totes awk $\leftarrow$ totally awkward - was excluded from the set of stimuli because the way totes is formed from totally deviated significantly from the rest of the words of the same structural type). The remaining three groups are smaller because these forms are 
extremely rare, and no other forms of the same kind could be found. Although they were included in the experiment, the main analysis focussed on the more strongly represented structural types. It should also be mentioned that WW blends were added to the experimental set at a later stage (see section 6.2.3), and therefore were not included in all analyses. Various characteristics of the target words were included in multifactorial analyses (see below) as item variables (see Appendix 5 for full list of item variables).

Table 8. Survey stimuli

\begin{tabular}{lll}
\hline Structural type & Number of stimuli & Examples \\
\hline AC & 14 & scigov $\leftarrow$ sci(ence) + gov(ernment $)$ \\
AD & 15 & weisure $\leftarrow$ w(ork $)+($ l)eisure \\
AW & 15 & celeblog $\leftarrow$ celeb $($ rity $)+$ blog \\
WD & 15 & jazzerina $\leftarrow$ jazz $+($ ball $)$ erina \\
WW & 15 & clapathy $\leftarrow$ clap + apathy \\
WC & 2 & blogfic $\leftarrow$ blog + fic $($ tion $)$ \\
BD & 2 & frohawk $\leftarrow($ a)fro $+($ m)ohawk \\
BC & 1 & netco $\leftarrow($ inter $)$ net + co(mpany $)$ \\
\hline total & 79 & \\
\hline
\end{tabular}

\subsubsection{Procedure}

The stimuli were presented on the Victoria University web portal in the form of a questionnaire that was designed using Qualtrics software (Version 44205 of the Qualtrics Research Suite). Each target word was presented in a context sentence either taken unchanged or adapted (identifying material such as personal names omitted or changed) from the source in which it had been found. The target word was printed in bold type, and the sentence was followed by the definition of the target word. Initially, the survey stimuli included only 64 target words, without WW blends, which were added at a later stage (see below). The 64 stimuli sentences were displayed in random order each time the survey was taken. The stimuli were preceded by an instruction, and each sentence was followed by a seven-point evaluation scale, as shown in (6.2). Four types of definitions of the target words were created, as exemplified in (6.3): W1W2 (the definition contains both the source words), W1 (only the first source word is included in the definition), W2 (only the second source word is included in the definition), and W0 (the definition does not include either of the source words). 
You will see a total of 64 sentences displayed successively on 10 pages. After each sentence there is a definition for the word in bold type. Please read the sentences and answer how successfully you think each definition explains the word by choosing the appropriate option.

Whether you are weathering the storm by building snowmen or plowing through piles of snow on your driveway, we are asking for photos of how you honor Old Man Winter. And if you want to stay in the comfort of your home, just post a photo of your backyard blizzaster.

blizzaster - a disaster caused by a blizzard

This definition is:

$\begin{array}{ccccccc}\text { Very poor } & \text { Poor } & \begin{array}{c}\text { Poor rather } \\ \text { than good }\end{array} & \begin{array}{c}\text { Neither } \\ \text { good nor } \\ \text { poor }\end{array} & \begin{array}{c}\text { Good } \\ \text { rather than } \\ \text { poor }\end{array} & \text { Good } & \text { Very good } \\ 0 & 0 & 0 & 0 & 0 & 0 & 0\end{array}$

a) globfrag -

- globalisation and simultaneous fragmentation (W1W2 definition)

- globalisation and simultaneous breakdown in connections between people (W1 definition)

- international integration and simultaneous fragmentation (W2 definition)

- international integration and simultaneous breakdown in connections between people (W0 definition)

b) blizzaster -

- a disaster caused by a blizzard (W1W2 definition)

- a sudden accident caused by a blizzard (W1 definition)

- a disaster caused by a snow storm (W2 definition)

- a sudden accident caused by a snow storm (W0 definition)

c) hydrail -

- hydrogen railway (W1W2 definition)

- trains that use hydrogen fuel (W1 definition)

- a railway system that uses highly flammable gas fuel (W2 definition)

- trains that use highly flammable gas fuel (W0 definition)

The order of the source words in the definition either reproduced the order of their parts retained in the target word (a), reversed it (b), or varied for different types of 
definitions (c). This was not manipulated purposefully, but was a consequence of expressing the meaning of a compound-like formation by a phrase. The reason for this is that syntagmatic origin blends are normally right-headed, as are most English compounds (Bauer 2009), and the definitions take the left head which is characteristic of phrases. The order of the source words of paradigmatic origin blends like (a) above, on the other hand, is not determined by semantics. The general intention was to make all the definitions sound as natural as possible (given the restrictions imposed by the definition type), and to explain the target word as successfully as possible, irrespective of the source word order and the number of source words retained. To achieve this, I asked 10 native speakers of English to read the full list of sentences with the stimuli and with all four definitions for each target word and to comment upon how successfully they thought the definitions explained the meaning of the target words. The feedback and comments from the testers were taken into consideration, and some definitions were rephrased. Even these measures could not, however, guarantee that the glosses are equivalent to the words they replace. There remains a possibility that the judgements about the quality of definitions might be affected not by the presence of the source words, but by some other factor influencing the appropriateness of the gloss, which is not accounted for in this study. Nevertheless, because there is no obvious way of measuring the appropriateness of the gloss and because the judgements regarding it are likely to be subjective, it seems appropriate to focus on the influence of the experimental factor, i.e. the presence of the source words in the definition, and to assume that all other influences can be treated as random item factors.

Four groups of stimuli were created, so that each target word had a definition from one of the four types in each group, and so that each group contained equal numbers of W1W2, W1, W2 and W0 definitions. The participants were randomly assigned to one of the four groups $(27,28,28$ and 29 participants, out of the 112 whose responses were included in the analysis). The survey was run with 64 stimuli (only two-element blends or clipping compounds that included some degree of shortening, i.e. AC, AD, AW, WD, WC, BD, and BC forms, see Table 1 above). Preliminary results of the study suggested that that it would be valuable to also assess fully overlapping WW blends because it appeared that the full preservation of source words was emerging as a significant factor influencing the evaluation of blend definitions. An additional survey was therefore created with just the $15 \mathrm{WW}$ target words and their definitions. The methods of presenting the stimuli and assigning them to the groups according to the definition type 
were exactly the same as described above. An e-mail was sent to the participants of the previous survey (99 e-mails were collected for the purpose of the prize draw, the names of the addressees were not known to the researcher) asking them to answer 15 additional questions. Out of the 99 participants that received the invitations, 58 completed the survey. No responses had to be excluded from the analysis, so all 58 were included. Because the email addresses in the new survey were not connected to the demographic data in the old survey (for the sake of anonymity), the demographic questions were included only in the main survey. Therefore, the information about the participants' age group, sex and linguistics education could not be used in the analyses which included WW blends, but all analyses included participant as a random factor.

\subsubsection{Methods of analysis}

It is often the case in linguistic studies that the effect of the experimental conditions can be quite hard to estimate because of the presence of many other effects which are not part of the experimental design. In this survey, the readers can evaluate the definitions of target words as more or less appropriate depending on many factors, such as the length of the blend itself and its source words, their frequency, orthographic or phonetic similarity, and many other factors which are not experimental variables. A way to approach this situation is to control for as many factors as possible by including the known properties of target words as factors in a statistical analysis. So, a number of characteristics of target words, and also demographic information about the participants were included in the analyses presented below. In the course of the analysis, it was sometimes necessary to choose between various ways to define certain item characteristics. Thus, various measures of similarity of blend words to their source words were used, including the orthographic similarity measure described in van Orden (1987). However, a simpler method of calculating the degree of similarity that is described below turned out to produce the predictor variable which outperformed other measures of similarity. Similarly, the method of calculating the average similarity between the source words and the blend by Levenshtein distance (see the discussion of item variables below) was selected as the most effective from several methods.

The statistical analysis of the survey results involved two qualitatively different approaches: conditional inference tree and mixed effects multiple regression modelling. The conditional inference tree (decision tree) method was used at various stages of the analysis, in order to estimate the hierarchy of independent variables that have 
significant influence on the dependent variable. This methodology was discussed in Chapter 4 (see also Hothorn et al. (2006) for more details). The results of the decision tree analysis were then used to inform the selection of factors in the regression analysis.

The influence of the two experimental variables on the dependent variable (alongside the influence of other item and participant factors) was estimated in a series of mixed effects regression models. For regression modelling, the dependent variable was transformed from a scalar value (having seven possible levels ranging from 'Very poor' to 'Very good') to a numeric value ranging from 1 to 7 (1 standing for 'Very poor', and 7 for 'Very good'). It has to be noted that presenting the scalar dependent variable in the form of a numeric one is an approximation, but this approximation provides a means of building more easily interpretable regression models, with more degrees of freedom (the latter is especially important when including interactions in the models). The assumption which underlies the decision to present the scalar variable as a numeric one is that there is an underlying continuous value (in this case, the 'goodness' of definition) which is indirectly measured on the scale used in the questionnaire. The relationship between this underlying variable and the independent variables will therefore be analysed in the regression models (see Bock and Diday (2000) for a discussion of such an approach to scalar variables).

The role of each factor and of possible interactions of factors was estimated by building a series of regression models which included different independent variables and by selecting the models that best predict the observed results. To separate out the effect of the experimental variables from all the other effects, I used a two-step approach to regression modelling (Hofmeister 2011). First, all variables except the experimental ones were used to build a series of regression models, and a model which best explained the influence of different factors on the response was selected out of the series. At the second stage a new model was built which used the residuals from the first model as the dependent variable, and which was used to estimate the influence of the experimental conditions on it. All statistical analyses were performed using the R software package (R Development Core Team 2012).

\subsection{Results and discussion}

The analysis below is based on responses received from 112 participants to the 64 items of the main survey, and from 58 participants to the 15 items of the additional survey. 
The analysis of the distribution of responses showed that, overall, the evaluations of the definitions of different target words vary dramatically, for example, the definitions of carbage $\leftarrow$ car + garbage were generally evaluated as 'Good' or 'Very good' and the definitions of acatramp $\leftarrow$ academic + trampoline received a lot of 'Poor' and 'Very poor' responses. The results of the analysis of participant and item factors that influence the responses will be presented below. First, the overall tendencies will be presented, and then the details of the statistical tests confirming their significance will be given.
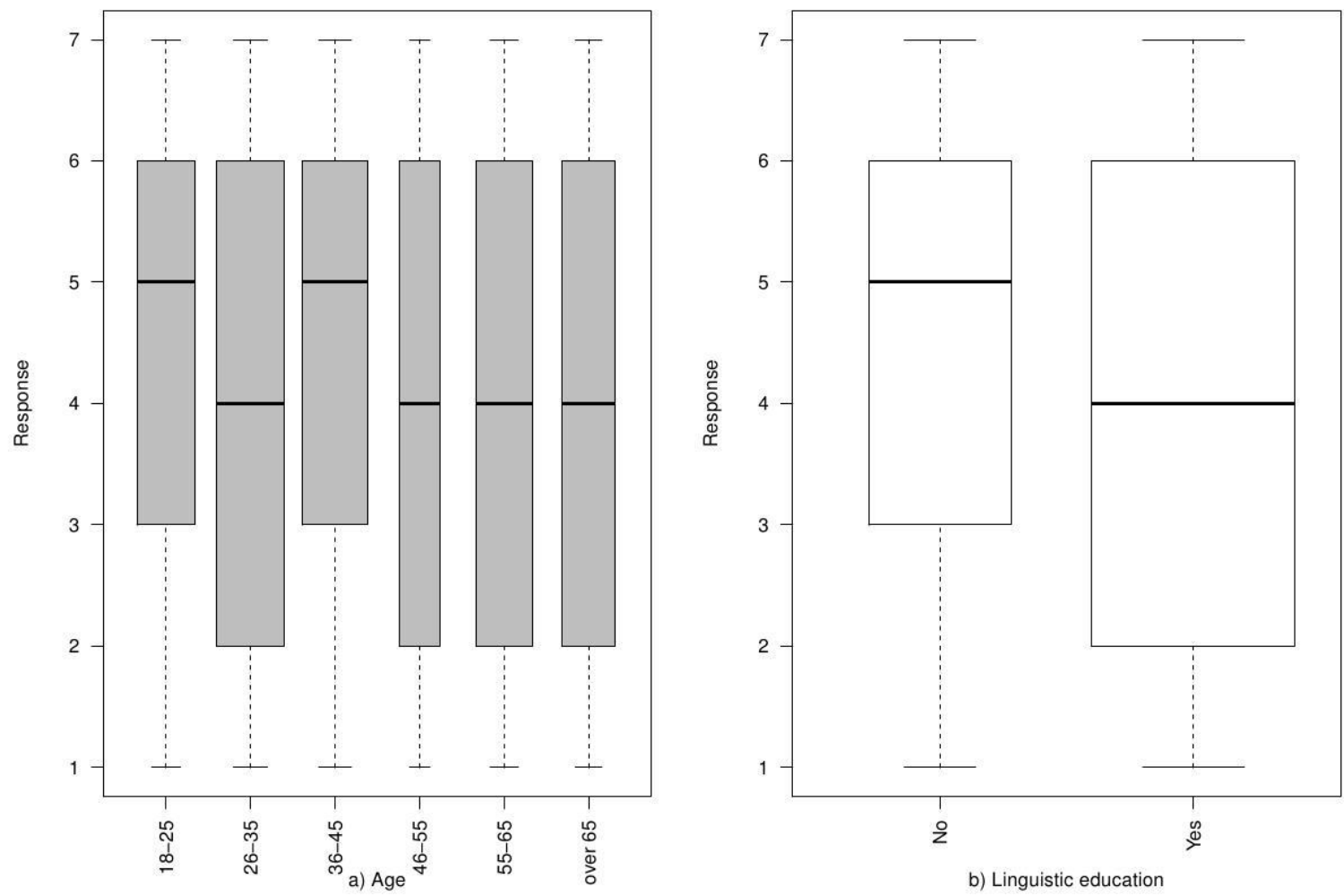

Figure 12. The distribution of responses by the age group of the participants (a) and by their education level (b). The width of the boxplots is proportional to the square root of the size of the group. The response values are labelled as 1-7 which corresponds to 'Very Poor' - 'Very Good'. The box plots show median responses for each category, as well as upper and lower quartiles.

The age of the participants and whether or not they have some background linguistic education turned out to influence the responses (Figure 12). The respondents with some background linguistic education tend to give more varied evaluations, and in particular, more lower grades. Most of the age groups have approximately the same distribution of responses, apart from the (18-25) and the (36-45) groups. The respondents in these two groups tend to give higher evaluations to the targets. The significance of the effects of age and education was confirmed in further analyses (see below). No significant influence of sex was revealed. 
In terms of the structural type of the target words, the median response for the definitions of AW, WD and WW forms is higher than for the definitions of AC and AD forms (Figure 13a). In terms of definition type (Figure 13b) the responses tend to be lower for the definitions containing no source words (W0) than for definitions containing one of the source words (W1 or W2) or both (W1W2).
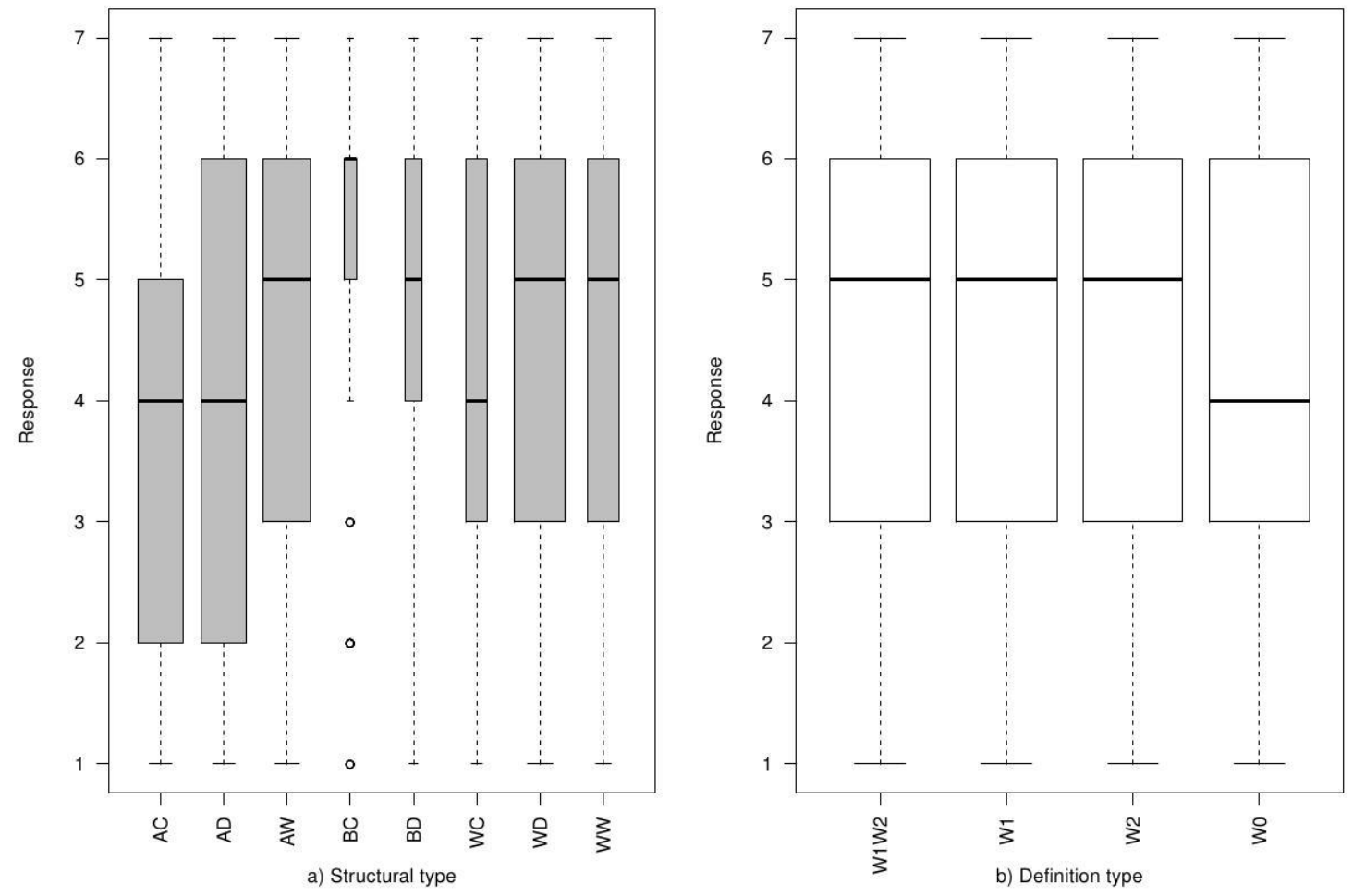

Figure 13. The distribution of responses by structural type of the target word (a) and by definition type (b).

The observed median responses differ across definition types in ways that reflect differences between the structural types of the target words. For AW, BC, WD and WW blends the median responses are the same for all four types of definitions, whilst the definitions of $\mathrm{AC}, \mathrm{AD}, \mathrm{BD}$ and $\mathrm{WC}$ target words are given higher evaluations if they contain the source words (see Table 9).

The results of an ANOVA show that both the effect of blend type and that of definition type on the response is significant $(F[4,6872]=69.58, p<0.0001$ for blend type; $F[3$, 6872] $=6.06, p=0.0004$ for definition type). Moreover, the effect of the interaction of blend type and definition type on the response is also significant $(F[12,6872]=2.35$, $\mathrm{p}=0.0052$ ). It has to be noted, however, that the observed effect could be due to factors other than blend type or definition type, or could be a cumulative effect of several different factors. This is especially important in a situation when, as in the present study, 
the stimuli are very diverse not only in terms of structural types but also in terms of frequencies, lengths and other characteristics. Therefore, in what follows, the influence of a number of item and participant factors on the readers' response will be considered alongside the influence of blend type and definition type discussed above. First, an exploratory analysis by a decision tree method will be carried out to estimate the relative influence of various factors on the response, and then the influence of blend type and definition type will be reconsidered in a multiple regression analysis.

Table 9. Observed median responses to definitions of different types of target words

\begin{tabular}{lllll}
\hline Blend type & \multicolumn{3}{l}{ Median response to definitions } & \\
\cline { 2 - 5 } & W1W2 & W1 & W2 & W0 \\
AC & 4 & 4 & 4 & 3 \\
AD & 5 & 4 & 4 & 4 \\
AW & 5 & 5 & 5 & 5 \\
BC & 6 & 6 & 6 & 6 \\
BD & 6 & 5 & 5 & 5 \\
WC & 5 & 4 & 4 & 4 \\
WD & 5 & 5 & 5 & 5 \\
WW & 5 & 5 & 5 & 5 \\
\hline
\end{tabular}

The influence of different factors on the participants' responses can be visualised in a decision tree. As mentioned in Chapter 4, this method estimates a regression relationship between the variables (both continuous and categorical) by splitting the data into significantly different 'branches' according to factors which have the most influence for each particular split (these factors are shown as nodes in the tree). In the following analysis, the conditional inference trees were built to visualise the effect of a number of predictor variables on the participants' responses. The following characteristics of target words were used as item predictor variables (see also the full list of variables in Appendix 5):

- the length of splinters (initial and final) in letters;

- the length of W1 and W2, each residualised against the splinter length (that is, estimating the effect of the length of the source words not already accounted for by the splinter length);

- the frequency of the source words in COCA: log transformed frequencies were used for the analysis, but raw frequencies are displayed in the plots below, to simplify the interpretation of the results; 
- the log transformed frequency of the blend word measured by the number of hits in Google search (as, for obvious reasons, COCA frequency could not be used for blends - see the discussion of data collection in section 4.1);

- the semantic type of the target word, that is, whether it is of paradigmatic origin, e.g. weisure (meaning both work and leisure)or of syntagmatic origin, e.g. intellidating (meaning 'intelligent dating'), as discussed in section 4.1;

- the orthographic similarity of the source words to the target words, i.e. the number of letters that the target word, e.g. advergame shares with the source word, e.g. advertisement, divided by total number of letters in the source word, calculated separately for W1 and W2;

- the average string edit distance (ASED) between the source words and the target word, also known as Levenshtein distance (Levenshtein, 1966), and calculated in the following way (Gries, 2012): (W1 $\rightarrow$ Blend + W2 $\rightarrow$ Blend) /2, i.e. the average of the number of letters that have to be inserted/deleted/substituted to turn W1 into the target word plus the number of insertions/deletions/substitutions that are necessary to turn W2 into the target word;

- whether the target word preserves the prosodic pattern (i.e. the number of syllables and the main stress position) of $\mathrm{W} 1$ or $\mathrm{W} 2$, or none of them;

- the frequency position of the source words in COCA among all the words beginning/ending with the letter string in the splinter (that is, whether the source words are the most frequent among all the words in COCA beginning or ending with that letter string, see also Appendix 5 for details);

- the COCA frequency of the coordinative combination (with and, or, or a comma) of the source words of the target word;

- the COCA frequency of the subordinative combination of the source words of the target word

- the structural type of the target word, i.e. AC, AD, AW, WD, or WW;

- the type of the definition, i.e. W1W2, W1, W2, or W0.

In addition to the item variables, the participants' age, sex, and background linguistic education were included in the analysis as participant variables. However, the participant variables could only be accounted for when analysing the data from the main survey (that is, the responses to the 64 target words of $\mathrm{AC}, \mathrm{AD}, \mathrm{AW}$ and WD types). The analysis including the data from the additional survey on $15 \mathrm{WW}$ blends included only item variables. 
Not all variables that were initially included in the analysis actually appear in the decision trees and in the regression models. Firstly, the decision trees do not display variables which do not have significant influence on the dependent variable. Secondly, some of the variables considered for including in regression models highly correlated with each other, which meant that some steps had to be taken in order to de-correlate them. In the process of regression model building, some predictors were residualised against one another or entirely excluded for this reason (see Dormann et al. (2013) for this approach to reducing multicollinearity in the data). Random intercepts for items and participants, and also random slopes where relevant, were added to the regression models because they account for item and participant properties not measured directly.

To save space, the responses are presented in the plots below on a scale from 1 to 7 , rather than on a scale from 'Very poor' to 'Very good'. However, it should be kept in mind that the response in this study is not a continuous measure like, for example, height or temperature, but, rather, an ordinal variable. This means that each response is a value on a scale with presumably unknown distances between the neighbouring values (it is assumed, for instance that 'Very good' is a higher value than 'Good' and 'Good' is a higher value than 'Good rather than poor', but it is not known whether the distance between the first pair of values is equal to the distance between the second pair). For these reasons, the type of the decision tree analysis below was ordinal regression, and therefore the results displayed in Figures 14-16 are not in terms of mean values, but, rather, in terms of distributions of the responses shown as bar plots in the terminal nodes of the trees.

In what follows, the analysis of the combined data from the main and the additional survey will be presented. Therefore, the focus will be on the item factors because the participant statistics was not collected in the additional survey and therefore could not be included in the combined data analysis. In addition to the combined data analysis, the analysis of the main survey only will be presented, to estimate the influence of the participant factors. 


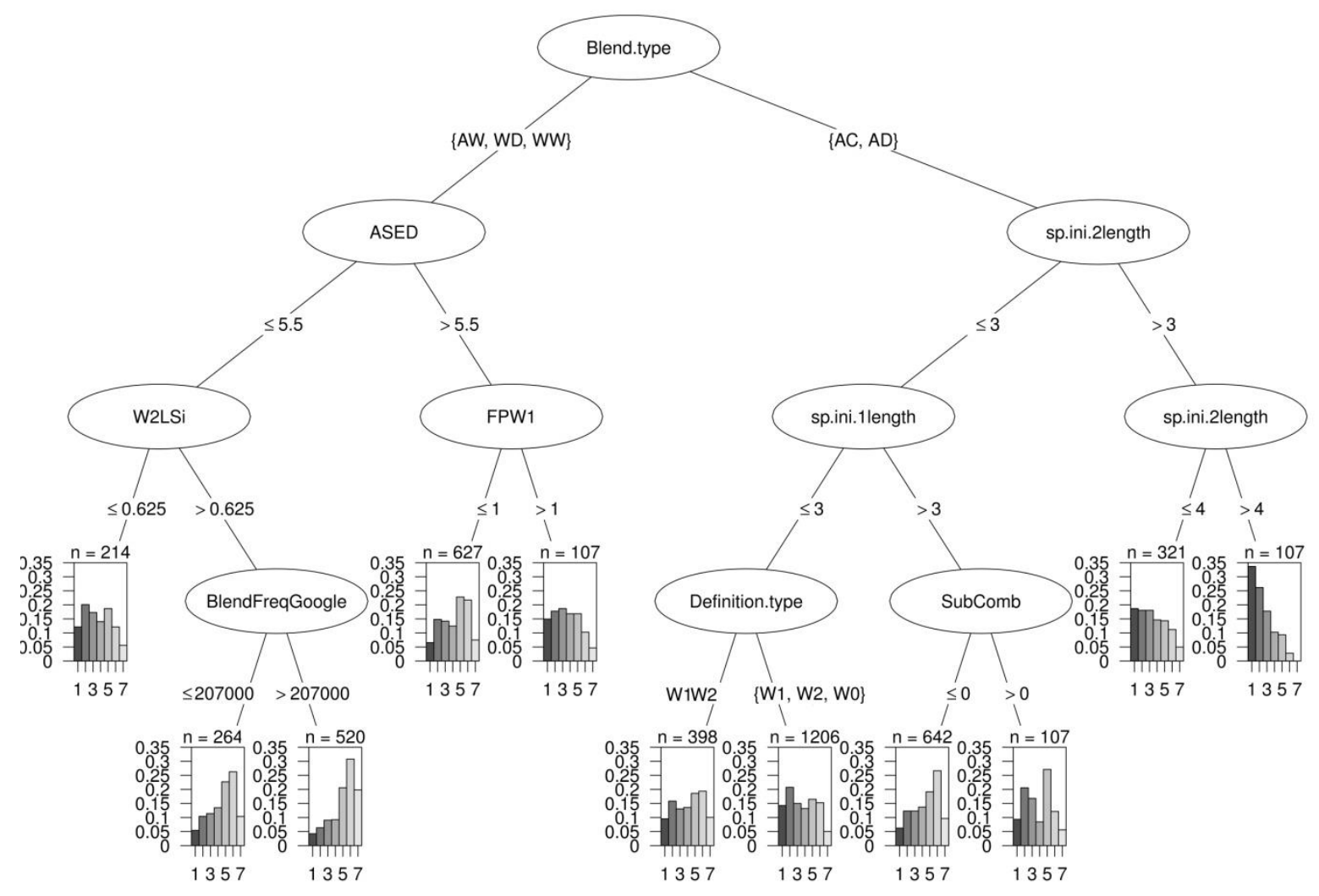

Figure 14. A decision tree analysis of the response with item factors as independent variables. Each node is labelled by the factor that works as the best predictor for it (from top to bottom): Blend.type - the structural type of the blend, ASED - average string edit distance between the source words and the target word, sp.ini.2length - the length of the initial splinter of W2, W2LSi-graphical similarity between W2 and the blend, FPW1 - frequency position of W1 in COCA (see Appendix for details), sp.ini.1length - the length of the initial splinter of W1, BlendFreqGoogle - the Google frequency of blend word (log transformed for all the analyses, raw frequencies are displayed in node labels for better interpretability), Definition.type - type of definition, SubComb - the frequency of cooccurrence of W1 and W2 in COCA as a subordinative word combination. The branches coming from each node are labelled by the values of the relevant factors for which the split is significant; $n$ is the number of responses in the terminal node, $p<0.05$ for each node. The bar plots show the distribution of responses from $1=$ very poor to $7=$ very good, for each terminal node.

As shown in Figure 14, blend type has the strongest influence on the participants' response because this factor appears in the topmost node of the tree. This is consistent with hypothesis 1 in section 6.1. Blends of WW, AW and WD types are assigned to the left branch with higher evaluations (the responses in left branches of the tree tend to be higher than in right branches, which is in accordance with the overall median responses for each structural type displayed in Figure 13a). This means that if a blend is formed by merging together two words so that they overlap, as in predictionary $\leftarrow$ prediction + dictionary, or if one of the source words is fully preserved, as in carbage or advergame $\leftarrow$ advertisement + game, then the definitions of such words generally received high evaluations. On the other hand, the definitions of blend words which do not preserve 
any of their source words in full, such as weisure $\leftarrow$ work + leisure, and of clipping compounds such as scigov, were less frequently evaluated as good or very good. Therefore, $\mathrm{AD}$ and $\mathrm{AC}$ forms appear in the right branch of the tree with lower evaluations.

A number of other predictors appear in the tree which means they also have a significant effect on the response (the higher the node in the tree the greater predictive power the corresponding variable has). The numbers displayed on top of terminal nodes indicate the number of responses in each node. The nodes with small $\mathrm{n}$ are informative as descriptive statistics, rather than as a basis for generalisations. More valuable information can be drawn from analysing nodes with larger $\mathrm{n}$ and tracking down (or up) the tree in order to see which factors have a significant influence on the responses to larger groups of target words. For instance, the responses to AW, WD and WW blend definitions appear to depend on the mean similarity of the two source words to the blend (ASED) and the frequency position of W1 (FPW1). The definitions of AW, WD and WW blends which are relatively similar to their source words, i.e. with ASED less than 5.5 (e.g. intellidating $\leftarrow$ intelligent + dating), tend to receive higher evaluations than those of blends which are relatively less similar to both the source words (ASED greater than or equal 5.5). In the latter group, higher evaluations are received if $\mathrm{W} 1$ of the blend is the most frequent word among all the words beginning with a particular letter string: this is measured by frequency position (FPW1, e.g. 1 for advergames $\leftarrow$ advertisement + games, and 15 for chofa $\leftarrow$ chair + sofa). For ASED=5.5 or less orthographic similarity of W2 to the target word (W2LSi) is significant: for less similar words (W2LSi=0.625 or lower), lower evaluations are given.

The evaluations of definitions of AC and AD target words (right branches of the tree) appear to be sensitive to a different set of factors than those of AW, WD and WW blends. These factors are the length of the initial splinter of W2 (sp.ini.2length) and W1 (sp.ini.1length), and, for the blends with both initial splinters of 3 letters or less, the type of definition (Definition.type). For a subset of the data corresponding to 1602 responses (the sum of 396 and 1206 in the two terminal nodes under the Definition.type node), W1W2 definitions receive fewer 'Very poor' / 'Poor' responses and more 'Good' responses than the other three types of definitions. The relevant nodes include 9 clipping compounds out of 14 , and 7 AD blends out of 15 , and all of them preserve fewer than 3 graphemes of the beginning of the first source word (that is, the length of the 
initial splinter sp.ini.1length is less than 3 as in briet $\leftarrow$ bride + diet, and foco $\leftarrow$ food + court). For example, the W1W2 definition of rumint 'rumour intelligence' was more often evaluated as 'Good' or 'Very good' than other definitions of the same word. The rightmost nodes in the tree include clipping compounds which combine relatively long parts of their source words (longer than 3 graphemes), such as globfrag $\leftarrow$ globalisation + fragmentation. The definitions of these target words generally received low evaluations whether the source words were present or not.

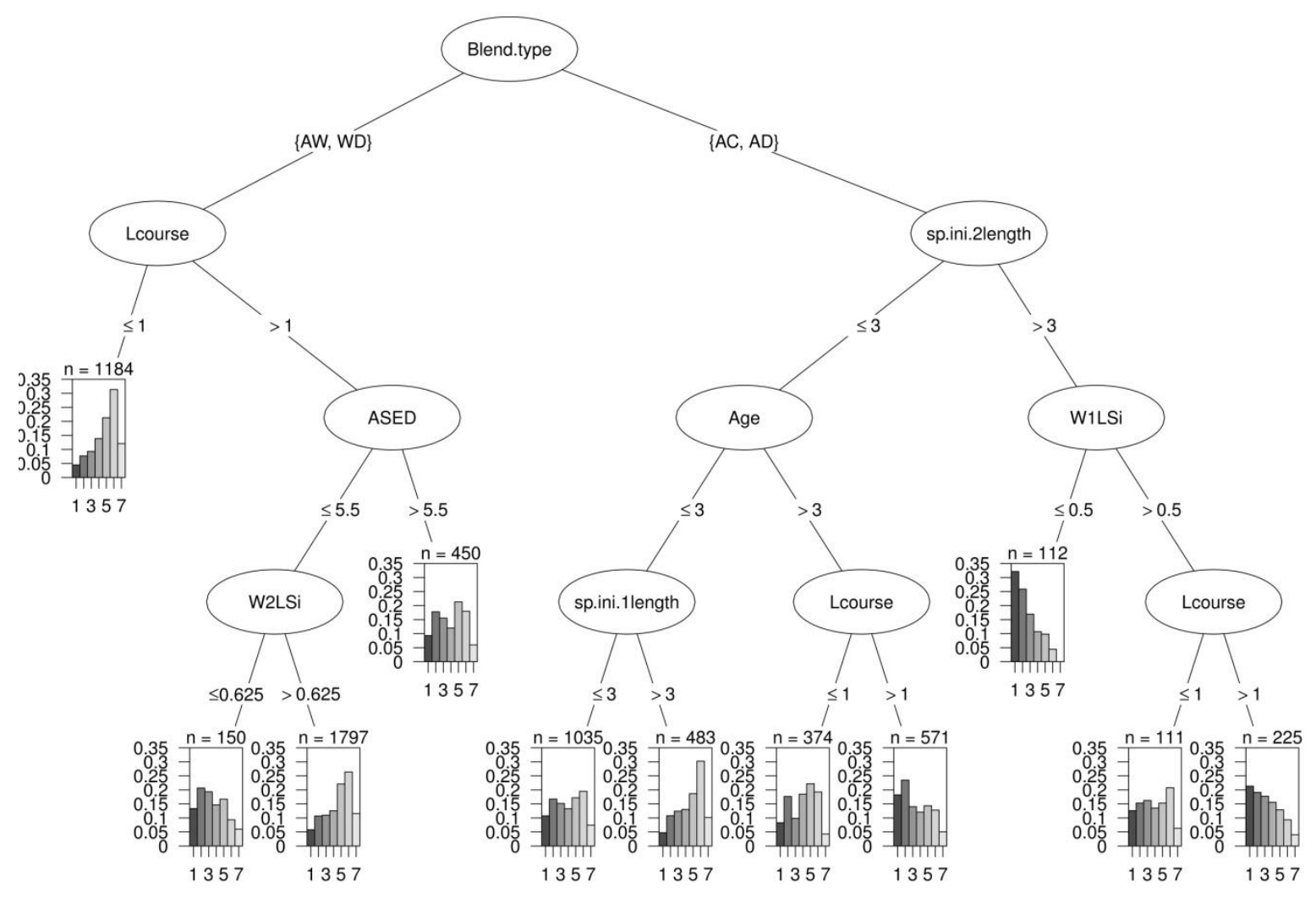

Figure 15. A decision tree analysis of the response to the main survey, with item and participant factors as independent variables.

Each node is labelled by the factor that works as the best predictor for it (from top to bottom): Blend.type - the structural type of the blend, Lcourse - whether or not the participant took a course in linguistics, sp.ini.2length - the length of the initial splinter of W2, ASED - average string edit distance between the source words and the target word, Age - the participants' age group, W1LSi-graphical similarity between W1 and the blend, W2LSi-graphical similarity between W2 and the blend, sp.ini.1length - the length of the initial splinter of W1. The branches coming from each node are labelled by the values of the relevant factors for which the split is significant; $n$ is the number of responses in the terminal node, $p<0.05$ for each node. The bar plots show the distribution of responses from $1=$ very poor to $7=$ very good, for each terminal node.

I focussed here on presenting results from joint data from the main and the additional survey, including all the structural types of target words. The decision tree analysis restricted to the main survey data only (Figure 15) shows a significant effect of the participants' age and education: older respondents and also those who had some 
linguistic education tend to give lower evaluations to the definitions, which is compatible with the distributions displayed in Figure 12 above.

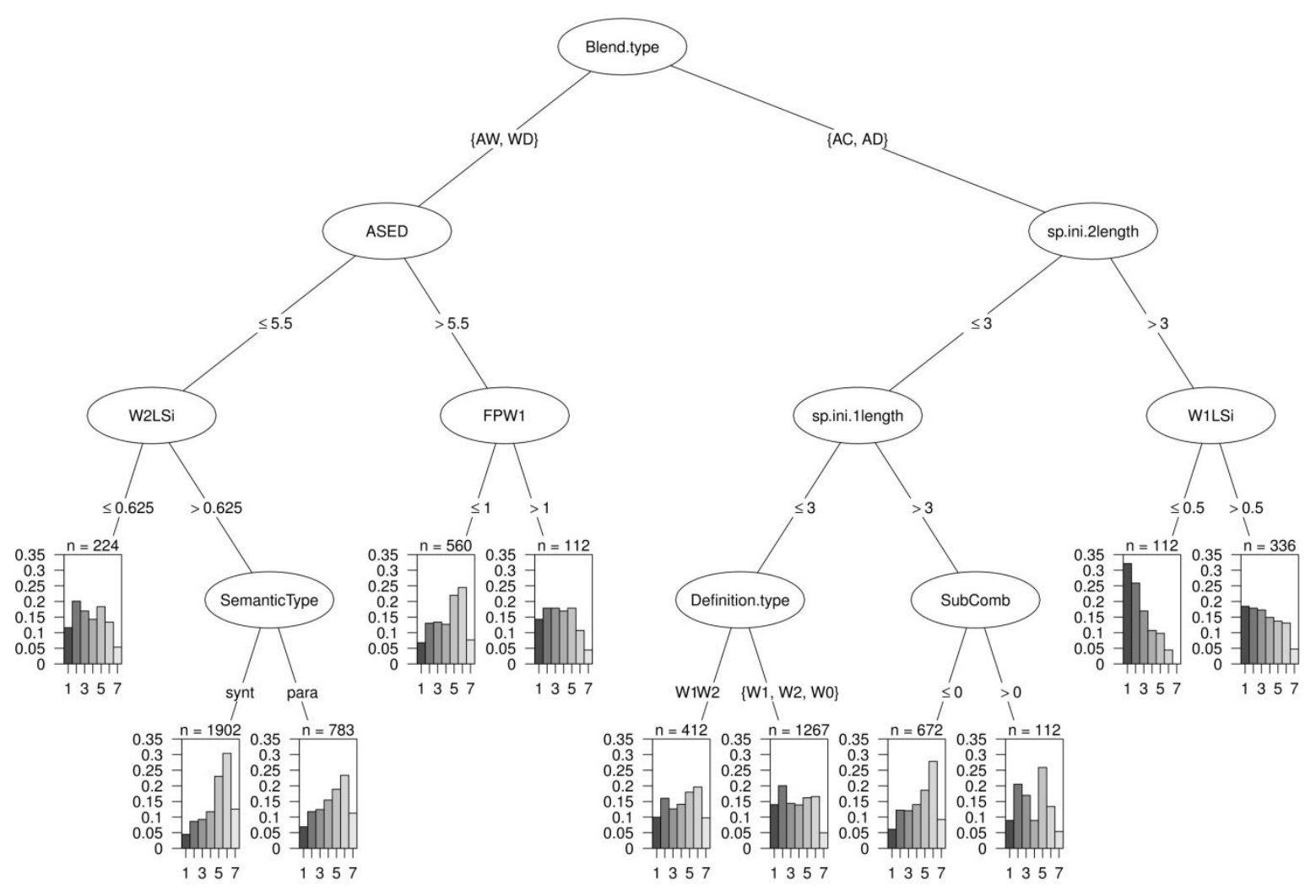

Figure 16. A decision tree analysis of the response to the main survey, with item factors as independent variables.

Each node is labelled by the factor that works as the best predictor for it (from top to bottom): Blend.type - the structural type of the blend, ASED - average string edit distance between the source words and the target word, sp.ini.2length - the length of the initial splinter of W2, W2LSi-graphical similarity between W2 and the blend, FPWI - frequency position of W1 in COCA, sp.ini.1length - the

length of the initial splinter of W1, WILSi-graphical similarity between W1 and the blend,

SemanticType - whether the target word is of syntagmatic (synt) or paradigmatic (para) origin,

Definition.type - type of definition, SubComb - the frequency of co-occurrence of W1 and W2 in COCA as a subordinative word combination. The branches coming from each node are labelled by the values of the relevant factors for which the split is significant; $n$ is the number of responses in the terminal node, $p<0.05$ for each node. The bar plots show the distribution of responses from $1=v e r y$ poor to $7=v e r y$ good, for each terminal node.

The results of the analysis shown in Figure 15 confirm that blends containing one of the source words in full (AW and WD) receive higher ratings than blends containing only clipped forms (AD and AC). The effects of the splinter length and the orthographical similarity between the source words and the blend are also observed in the main survey analysis. The definition type, on the other hand, does not appear in the tree, which means that the effect of the definition type is no longer significant once the effects of participant variables are accounted for. To check this, another decision tree of the main 
survey data was built, this time with item predictor variables only. The decision tree in Figure 16 is very similar to the one in Figure 14, which is not surprising because most of the data used for the two trees are the same. It should be noted that among the item variables that turn out to be significant for the main survey data is the semantic type of the blend, and not frequency, as was the case with the joint data from the main and the additional survey. Another difference is in the rightmost node of the tree, corresponding to $\mathrm{AC}$ and $\mathrm{AD}$ blends with the initial splinter of more than 3 graphemes. The joint data tree (Figure 14) assigns the difference in the responses to the length of the initial splinter, while the main survey tree (Figure 16) assigns this difference to the similarity of W1 to the target word. This may be due to the fact that some significant differences in responses relevant to particular subsets of data can be explained by different factors, or could even be due to random properties of particular target words which cannot be accounted for in this analysis.

In sum, the decision tree analysis of the responses has shown that the structural type of the target word has a significant influence on how the definitions of the target words are evaluated. It has also shown that different sets of item factors are significant predictors of the responses for AW, WD and WW blends on the one hand, and for AD and AC target words on the other hand, and that the definition type appears to be significant only for a subset of the data. To better understand the role of the structural type of the target word and of the definition type as predictors of the response variable, a multiple regression analysis was performed. It is essential to complete the analysis above with regression modelling because the latter can account for random item and participant factors.

As mentioned in section 6.2.4, a two-step approach to regression modelling was applied in order to more carefully investigate the influence of two experimental variables on the response. First, a series of models was built to predict the response with regard to significant effects other than those of blend type and definition type. The models were built with the same independent item and participant variables as the tree models above, excluding the two experimental variables. As was the case with the variables in the tree analyses, W1 length and W2 length were residualised against the length of W1 splinters and W2 splinters, respectively, to avoid high correlations between the variables. Therefore, the variables 'w1length' and 'w2length' in the models below stand for the length of W1 and W2 not already accounted for by the length of the splinters. Random intercepts for items (qID) and participants (UID) were included in the model, as 
well as random slopes for items over the participants' age, and for linguistic background (the results of an ANOVA comparing the models with different structures of random effects are summarised in Table 10). The formula and the outcome of the final model are given in Table 11. The full model summary is presented in (A6.3), Appendix 6.

Table 10. Likelihood ratio tests comparing models predicting the response in the main survey with increasingly complex random effects structure

\begin{tabular}{lcccllll}
\hline & Df & AIC & BIC & $\begin{array}{l}\text { log- } \\
\text { likelihood }\end{array}$ & Chi-square & $\begin{array}{l}\text { Df for Chi- } \\
\text { square }\end{array}$ & p-value \\
\hline $\begin{array}{l}\text { random intercepts } \\
\text { only }\end{array}$ & 26 & 23800 & 23976 & -11874 & & & \\
$\begin{array}{l}\text { random slope for } \\
\text { items over age }\end{array}$ & 28 & 23796 & 23989 & -11872 & 5.1311 & 2 & 0.04688 \\
$\begin{array}{l}\text { included } \\
\text { random slopes for } \\
\text { items over age and }\end{array}$ & 31 & 23791 & 24002 & -11865 & 13.579 & 3 & 0.00353 \\
$\begin{array}{l}\text { linguistic } \\
\text { background } \\
\text { included }\end{array}$ & & & & & & & \\
\hline
\end{tabular}

Table 11. The effects of item and participant factors on the response in the main survey

\begin{tabular}{|c|c|c|c|}
\hline \multicolumn{4}{|c|}{$\begin{array}{l}\text { Model formula: } \\
\text { response } \sim \text { w1length + w2length + sp.ini.1length + sp.ini.2length + sp.fin.2length + w1freqCoca + } \\
\text { w2freqCoca + BlendFreqGoogle + W1LSi + W2LSi + ASED + ProsW } 1+\text { ProsW } 2+\text { FPW } 1+\text { FPW } 2+ \\
\text { CoComb + SubComb + Sex + Age + Lcourse + (1 } \mid \text { participant })+(1+\text { age } \mid \text { item })+(1+\text { Lcourse } \mid \text { item })\end{array}$} \\
\hline & Estimate & Std. Error & t value \\
\hline Intercept & 7.731 & 1.368 & 5.650 \\
\hline participant & -0.005840 & 0.002717 & -2.149 \\
\hline item & -0.03404 & 0.006879 & -4.949 \\
\hline w1length & -0.02651 & 0.04705 & -0.563 \\
\hline w2length & 0.08142 & 0.06490 & 1.255 \\
\hline sp.ini.1length & 0.02349 & 0.06531 & 0.360 \\
\hline sp.ini.2length & -0.4221 & 0.1264 & -3.339 \\
\hline sp.fin.2length & 0.04892 & 0.07609 & 0.064 \\
\hline w1freqCoca & $6.402 \mathrm{e}-07$ & $1.269 \mathrm{e}-06$ & 0.504 \\
\hline w2freqCoca & $-2.360 e-06$ & $2.200 \mathrm{e}-06$ & -1.072 \\
\hline BlendFreqGoogle & $-1.449 e-07$ & $1.245 \mathrm{e}-07$ & -1.164 \\
\hline W1LSi & -0.2390 & 0.5938 & -0.403 \\
\hline W2LSi & 0.1806 & 0.7796 & 0.232 \\
\hline ASED & -0.1423 & 0.07606 & -1.871 \\
\hline ProsW1 & -0.05672 & 0.1541 & -0.368 \\
\hline ProsW2 & -0.02870 & 0.2296 & -0.125 \\
\hline FPW1 & $7.405 \mathrm{e}-04$ & 0.004116 & 0.180 \\
\hline FPW2 & $8.277 \mathrm{e}-04$ & 0.004983 & 0.314 \\
\hline CoComb & -0.009070 & 0.008979 & -1.010 \\
\hline SubComb & -0.001489 & 0.002314 & -0.643 \\
\hline Sex & -0.09555 & 0.1882 & -0.508 \\
\hline Age & -0.1158 & 0.05218 & -2.219 \\
\hline Lcourse & -0.4603 & 0.1862 & -2.473 \\
\hline
\end{tabular}

To save space, the correlations between fixed effects are not displayed in Table 11, but it has to be noted that the absolute values of the coefficients of the correlation between different predictors used in the model are no larger than 0.25 . This means that the 
predictors selected for the model are relatively independent. The significance of the effects in the model in Table 11 is estimated using t-values because p-values cannot be extracted from models with random slopes. However, for large enough samples (n>30) an absolute t-value greater than 2 is a sufficient indicator of significance at the $5 \%$ level (Baayen and Milin, 2010). The model in Table 11 shows significant effects of the length of the initial splinter of W2 (i.e. 'sp.ini.2length', the regression estimate is $-0.4366, t=-$ 3.539), ASED (the regression coefficient is $-0.16, \mathrm{t}=-2.296$ ), the participants' age (the regression coefficient is $-0.116, t=-2.219$ ), and of their prior linguistic education 'Lcourse' (the regression coefficient is $-0.46, \mathrm{t}=-2.473$ ). These are the effects also shown in the upper nodes of the decision tree in Figure 15 above. The reliability of the model can also be tested by checking the distribution of its residuals (Figure 17). The distribution of the residuals of the model is very close to a straight line and thus is best approximated as normal, therefore it can be concluded that the model inference is valid.

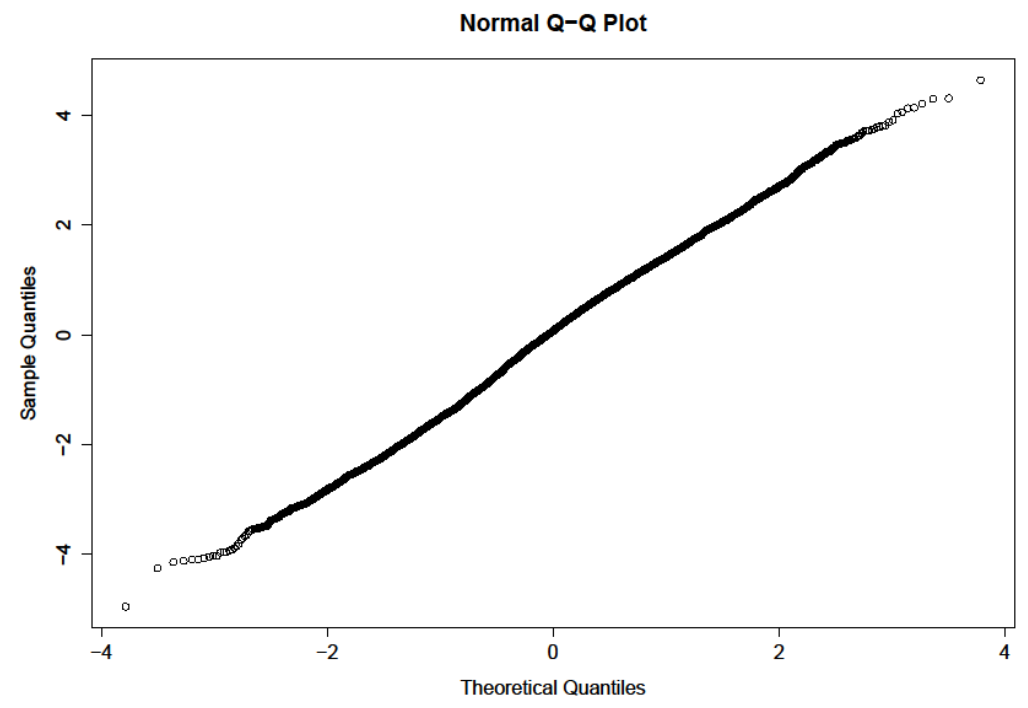

Figure 17. A quantile-to-quantile plot of the residuals of the regression model predicting the responses in the main survey.

Despite the fact that the model in Table 11 contains a number of non-significant factors, it was selected for the second step of the analysis as the one that best predicts the response out of the series of regression models. Compared to a model including only significant predictors from Table 11, the full model demonstrates a better fit to the data (Chi-square=6.1322, $\mathrm{Df}=2, \mathrm{p}=0.0043$ ).

The second step of the modelling was to generate residuals from the selected model and then to use these residuals as the dependent variable in models which only included the experimental factors as independent variables, together with random intercepts for 
items and participants. The reference level of the intercept for blend type is $\mathrm{AC}$, and for the definition type is W1W2, which means that the responses to different structural types of blends are compared to responses to AC target words, and the responses to different types of definitions are compared to responses to W1W2 definitions. AC type was selected as the reference intercept level for two reasons. Firstly, to compare the responses to clipping compounds (AC target words) to the responses to other target words was one of the objectives of this experiment. Secondly, the descriptive statistics and the decision tree analysis above suggests that the definitions of AC target words are most likely to receive lower evaluations, and therefore should be useful as a baseline for comparison. On the other hand, because it was hypothesised that the presence of the source words in the definition should increase the probability of higher evaluations (at least for some of the target words), it was assumed that W1W2 definitions should receive the highest evaluations, and therefore it would be reasonable to use W1W2 as the reference intercept level. This choice of reference levels of intercepts is used for all the models in this section.

Table 12. Likelihood ratio test comparing the model with simple effects of blend type and definition type with the model including the interaction of the two factors

\begin{tabular}{|c|c|c|c|c|c|c|c|}
\hline & Df & AIC & BIC & $\begin{array}{l}\text { log- } \\
\text { likelihood }\end{array}$ & Chi-square & $\begin{array}{l}\text { Df for Chi- } \\
\text { square }\end{array}$ & $\mathrm{p}$-value \\
\hline $\begin{array}{l}\text { simple effects of } \\
\text { blend type and } \\
\text { definition type }\end{array}$ & 10 & 22992 & 23060 & -11486 & & & \\
\hline $\begin{array}{l}\text { interaction of blend } \\
\text { type and definition } \\
\text { type included }\end{array}$ & 19 & 22985 & 23113 & -11473 & 25.766 & 9 & 0.00223 \\
\hline
\end{tabular}

6) shows the significant effects of definition type: the evaluations of W1, W2 and W0 definitions are significantly lower than those of W1W2 definitions (the regression coefficients are $-0.142,-0.118$ and -0.306 respectively, $t>2$ for all three definition types). No significant effect of blend type is shown by the model ( $t<2$ for all blend types). It is reasonable, however, to account for the interaction of the two factors because such an interaction is predicted by hypothesis 2 in section 6.1. The model that included the interaction of the two factors performed significantly better than the one including blend type and definition type as separate effects, as shown in the ANOVA comparison summarised in Table 12. The outcome of the model is summarised in Table 13. The intercept reference level of the model including the interaction is AC:W1W2, that is, W1W2 definitions of AC targets. The negative effect of W1, W2 and W0 definition types is confirmed by the model in Table 13 . 
Table 13. The effects of blend type and definition type on the response in the main survey

\begin{tabular}{|c|c|c|c|}
\hline $\begin{array}{l}\text { Model formula: } \\
\text { response } \sim \text { Blend.type + Definitiol }\end{array}$ & e * Definit & $+(1 \mid u I D)$ & \\
\hline & Estimate & Std. Error & tvalue \\
\hline Intercept & 0.35581 & 0.07415 & 4.799 \\
\hline Blend.typeAD & -0.14131 & 0.10590 & -1.334 \\
\hline Blend.typeAW & -0.34598 & 0.10132 & -3.415 \\
\hline Blend.typeWD & -0.31563 & 0.09835 & -3.209 \\
\hline Definition.typeW1 & -0.41423 & 0.10279 & -4.030 \\
\hline Definition.typeW2 & -0.40389 & 0.10701 & -3.774 \\
\hline Definition.typeW0 & -0.59966 & 0.10574 & -5.671 \\
\hline Blend.typeAD:Definition.typeW1 & 0.19448 & 0.14723 & 1.321 \\
\hline Blend.typeAW:Definition.typeW1 & 0.52019 & 0.14194 & 3.665 \\
\hline Blend.typeWD:Definition.typeW1 & 0.32190 & 0.13805 & 2.332 \\
\hline Blend.typeAD:Definition.typeW2 & 0.15342 & 0.15020 & 1.021 \\
\hline Blend.typeAW:Definition.typeW2 & 0.39668 & 0.14515 & 2.733 \\
\hline Blend.typeWD:Definition.typeW2 & 0.50463 & 0.14099 & 3.579 \\
\hline Blend.typeAD:Definition.typeW0 & 0.23251 & 0.15006 & 1.549 \\
\hline Blend.typeAW:Definition.typeW0 & 0.44264 & 0.14390 & 3.076 \\
\hline Blend.typeWD:Definition.typeW0 & 0.42868 & 0.13975 & 3.067 \\
\hline
\end{tabular}

The model also shows a significant difference in responses between AC targets and each of the AW and WD blend types, although the sign of the regression coefficient is different from what could be expected from the descriptive statistics and the decision tree analysis: the simple effects of AW and WD blend types have negative coefficients $(-0.346$ for AW and -0.316 for WD, the difference between AC and AD is not significant) indicating that the model predicts lower evaluations for the definitions of AW and WD blends compared to the definitions of AC forms. This result is due to the chosen intercept level, that is, the responses to AW, WD and AD blend types are compared to the responses to the W1W2 definitions of $\mathrm{AC}$ forms, and those tend to have higher evaluations than AC definitions on average. A model with AC:W0 intercept reference level showed positive coefficients for blend type as a simple effect, but the overall performance of that model was not as good as that of the model in Table 13, and therefore it is not discussed here.

Analysis of the regression coefficients of the interaction of blend type and definition type (visualised in Figure 18) shows that the responses to W1, W2, and W1W2 definitions are distributed between the four types of target words in a very different way. For AC and AD targets, the W1W2 definition is clearly the preferred kind, and the effect of W1W2 is stronger for AC than AD (at least numerically, if not significantly). In addition to this, it has to be noted that definitions of AW blends that include W1, and definitions of WD blends that include W2 are given significantly greater positive evaluations than W0 definitions of AC. It appears that if a source word (either W1 or W2) is not fully 
preserved in the blend then the participants become sensitive to its presence in the definition. Figure 18 shows that both for AW and for WD blends the definition which provides the source word which is not fully retained in the blend is the preferred type.

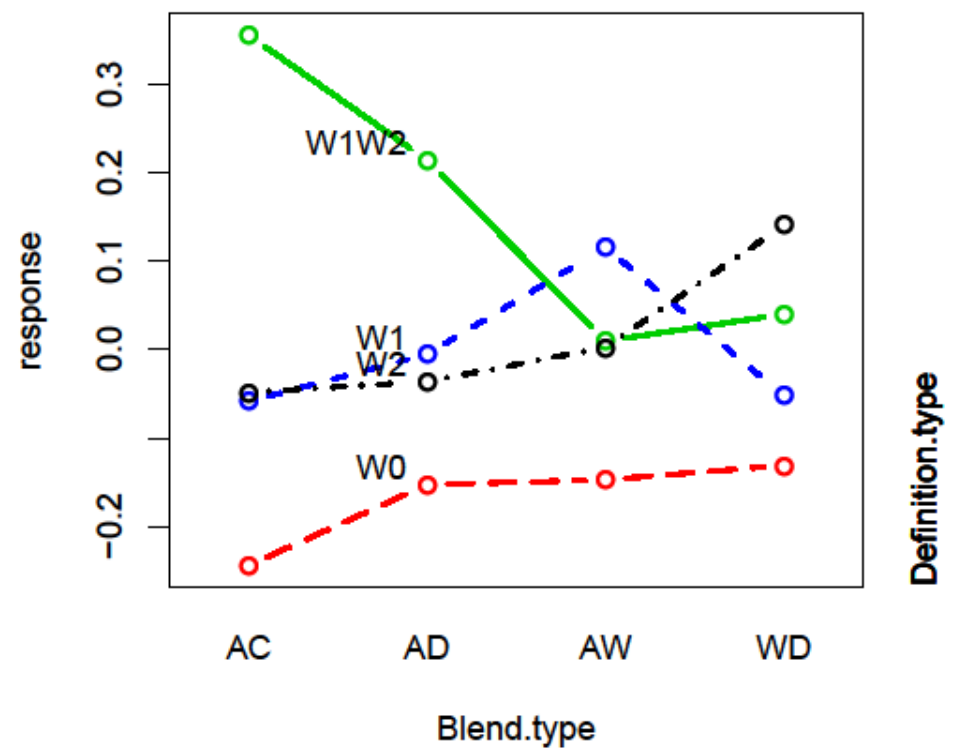

Figure 18. Regression coefficients for response by blend type and definition type, in the model with interaction of these experimental variables. Different line styles of the graphs correspond to different definition types: solid line to W1W2 definition, short dashes to W1 definitions, dots and dashes to W2 definitions, long dashes to WO definitions

Similar analysis was performed for the data including the responses to the additional survey. A series of models predicting the response as an outcome of item factors other than blend type and definition type were built as a first step or regression analysis. The item factors included in the model were the same as in the model in Table 11. The significant effects shown by the first-step model (A6.6 in Appendix 6) are those of the length of the initial splinter of W2 (the regression coefficient is $-0.242, t=-2.086$ ), the orthographic similarity of W1 to the blend (the regression coefficient is $0.889, \mathrm{t}=2.090$ ) and the random item and participant effects. No interactions between the item variables or any random slopes for item variables were justified. The residuals from the model were then used to build a model accounting for the effects of blend type and definition type. As was the case with the models above, the model including only simple effects of blend type and definition type was compared to the model including also their interaction, the results of the comparison are summarised in Table 14. 
Table 14. Likelihood ratio test comparing the model with simple effects of blend type and definition type with the model including the interaction of the two factors (combined data from the main and the additional survey)

\begin{tabular}{lccclccc}
\hline & Df & AIC & BIC & $\begin{array}{l}\text { log- } \\
\text { likelihood }\end{array}$ & Chi-square & $\begin{array}{l}\text { Df for Chi- } \\
\text { square }\end{array}$ & p-value \\
\hline $\begin{array}{l}\text { simple effects of } \\
\text { blend type and } \\
\text { definition type }\end{array}$ & 11 & 25016 & 25091 & -12497 & & & \\
$\begin{array}{l}\text { interaction of blend } \\
\text { type and definition } \\
\text { type included }\end{array}$ & 23 & 25005 & 25162 & -12480 & 34.702 & 12 & 0.00052 \\
\hline
\end{tabular}

Overall, the output of the regression models in Tables 15 and 16 predicting the responses to both main and additional survey is very similar to the output of the models accounting only for the main survey data (which is not surprising because a great part of the data used for all models is the same).

Table 15. Simple effects of blend type and definition type in the main and the additional survey

\begin{tabular}{llll}
\hline $\begin{array}{l}\text { Model formula: } \\
\text { response } \sim \text { Blend.type + Definition.type + (1|uID) }\end{array}$ & & & \\
\hline & Estimate & Std. Error & t value \\
\hline Intercept & 0.122380 & 0.050329 & 2.432 \\
Blend.typeAD & -0.010040 & 0.056266 & -0.178 \\
Blend.typeAW & -0.007946 & 0.053582 & -0.148 \\
Blend.typeWD & -0.011097 & 0.053577 & -0.207 \\
Blend.typeWW & 0.017108 & 0.069088 & 0.248 \\
Definition.typeW1 & -0.124132 & 0.050319 & -2.467 \\
Definition.typeW2 & -0.086284 & 0.050715 & -1.701 \\
Definition.typeW0 & -0.259037 & 0.050583 & -5.121 \\
\hline Intercept levels: Blend typeAC, Definition typeW1W2 & &
\end{tabular}

Table 16. The effects of blend type and definition type, and the interaction of the two factors in the main and the additional survey

\begin{tabular}{|c|c|c|c|}
\hline \multicolumn{4}{|c|}{$\begin{array}{l}\text { Model formula: } \\
\text { response } \sim \text { Blend.type + Definition.type +(Blend.type } * \text { Definition.type })+(1 \mid \text { uID })+(1 \mid q I D)\end{array}$} \\
\hline & Estimate & Std. Error & t value \\
\hline Intercept & 0.36195 & 0.07886 & 4.590 \\
\hline Blend.typeAD & -0.14014 & 0.11268 & -1.244 \\
\hline Blend.typeAW & -0.33244 & 0.10624 & -3.129 \\
\hline Blend.typeWD & -0.34681 & 0.10641 & -3.259 \\
\hline Blend.typeWW & -0.47334 & 0.13738 & -3.445 \\
\hline Definition.typeW1 & -0.42556 & 0.10953 & -3.886 \\
\hline Definition.typeW2 & -0.39733 & 0.11428 & -3.477 \\
\hline Definition.typeW0 & -0.61075 & 0.11259 & -5.424 \\
\hline Blend.typeAD:Definition.typeW1 & 0.20014 & 0.15675 & 1.277 \\
\hline Blend.typeAW:Definition.typeW1 & 0.49381 & 0.14917 & 3.310 \\
\hline Blend.typeWD:Definition.typeW1 & 0.36462 & 0.14933 & 2.442 \\
\hline Blend.typeWW:Definition.typeW1 & 0.48853 & 0.19334 & 2.527 \\
\hline Blend.typeAD:Definition.typeW2 & 0.10867 & 0.16044 & 0.677 \\
\hline Blend.typeAW:Definition.typeW2 & 0.37413 & 0.15270 & 2.450 \\
\hline Blend.typeWD:Definition.typeW2 & 0.52732 & 0.15250 & 3.458 \\
\hline Blend.typeWW:Definition.typeW2 & 0.63790 & 0.19570 & 3.260 \\
\hline Blend.typeAD:Definition.typeW0 & 0.22129 & 0.15992 & 1.384 \\
\hline Blend.typeAW:Definition.typeW0 & 0.43791 & 0.15096 & 2.901 \\
\hline Blend.typeWD:Definition.typeW0 & 0.45588 & 0.15120 & 3.015 \\
\hline Blend.typeWW:Definition.typeW0 & 0.84323 & 0.19528 & 4.318 \\
\hline
\end{tabular}


The regression analysis results confirm that, for AC target words, the definitions of the W0, W1 and W2 types are given significantly lower evaluations than the W1W2 definitions. It is also confirmed that the definitions of all the target words which preserve one or more of their source words in full (that is, WW, WD and AW) are evaluated higher than those of AC forms. The regression coefficients for the difference between responses to AD and AC target words have the same sign as for other blend types, but the effect is not significant at the $5 \%$ level.

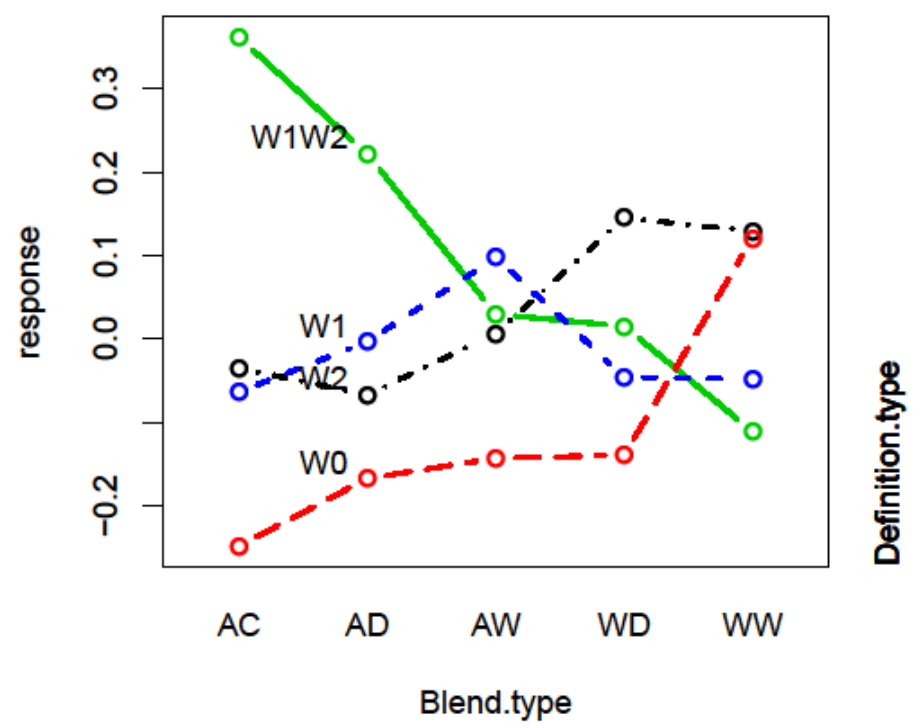

Figure 19. Regression coefficients for response by blend type and definition type, in the model with interaction of these experimental variables. Different line styles of the graphs correspond to different definition types: solid line to W1W2 definition, short dashes to W1 definitions, dots and dashes to W2 definitions, long dashes to WO definitions

The effect of the interaction between blend type and definition types visualised in Figure 19 is similar to those in Figure 18. In addition, it can be seen that the definitions containing none of the source words (W0) are given less positive evaluations for all the target words except WW blends. This can be explained by the fact that the source words can easily be accessed from the form of WW blends and therefore there is no need to include them in the definition. Moreover, W1W2 definitions are the least preferred for WW blends, according to the model. It may be the case that a definition that includes the source words which are already present in the blend itself makes the definition look redundant. Interestingly, W2 definitions of WW blends are given the same amount of high evaluations as W0 definitions of these words, and both W2 and W0 definitions appear to be preferred to W1 or W1W2 definitions. It appears that the presence of only W2 in the definitions of WW blends does not result in lower evaluations of such definitions. A possible explanation is that W2 is less salient than W1 in the form of WW 
blends because the beginning of W2 does not coincide with the beginning of the blend, and therefore may not be easily recognised as a word onset (in fact, some of the findings in Chapter 7 suggest this is the case). The fact that including the interaction between blend type and definition type has significantly improved the model fit implies that whether or not the evaluation of the target words' definition is sensitive to the presence or absence of their source words, depends on the type of the target words. The decision tree analysis described above demonstrates qualitative characteristics of the target words which are more successfully explained by definitions containing both the source words - these are clipping compounds preserving relatively small portion of the graphical material of their source words. The outcome of the regression analysis provides a quantitative estimate of these effects.

\subsection{Interim conclusions: Perception and understanding of blends and clipping compounds}

The survey results strongly support the hypothesis that the structural type of the blend/clipping compound influences the readers' evaluation of its definition (hypothesis 1). The results also suggest that, when evaluating the definitions of target words, the readers appear to be sensitive to their structural type. It was confirmed that the readers give higher evaluations to less formally transparent target words if their source words are included in the definitions (hypothesis 2). It would be intuitively clear that any blend word or clipping compound is easier to explain by using the words it is made of, rather than by paraphrasing them. What is striking about the results of this survey is that this factor turns out to have an impact on the definitions of a subset of words with specific characteristics. If the source words cannot be easily recovered from the form of a blend or a clipping compound (as it appears to be the case for weisure and scigov), then it is essential to use these words in order to explain the meaning of the blend or clipping compound. This is also true for cases when only one source word cannot be recovered from the blend, as the readers give numerically higher evaluations to $\mathrm{W} 1$ definitions of AW blends and W2 definitions of WD blends. That is, the readers seem to prefer definitions in which the full counterparts of the incomplete splinters of blends are provided, as in the W1 definition of approximeeting which includes the word approximate, and in the W2 definition of complimentsult which includes the word insult. On the other hand, if the source words are recoverable well enough from the blend itself, as in the case of predictionary, their presence in the definition becomes less important because the 
formal transparency of the blend enhances the recoverability of their source words. The results of the survey reveal that the structural type of target words explains the variance in the response to a considerable extent. The construct of the structural type introduced in Chapter 4 reflects two aspects of blend formation: formal transparency (that is, full versus partial preservation of the source words and preserving word beginnings versus word ends) and, albeit indirectly, recognisability of the source words. The results above confirm that full preservation of the source words (which is true for AW, WD and WW blends) makes the respondents less sensitive to the types of definition, which clearly distinguishes AW, WD and WW target words from the ones which do not preserve the source words in full (i.e. AD or AC). Having said this, I must admit that different patterns are observed for AW, WD and WW blends with respect to $\mathrm{W} 1$ and $\mathrm{W} 2$ definitions. This means that full preservation of only one of the source words influences the evaluation of the definitions depending on the presence of that source word in them.

The participants of the survey were asked to evaluate how well each definition explained the highlighted words. This instruction could potentially be interpreted in two different ways. On the one hand, the participants could have interpreted it as an invitation to judge how well the given definitions matched their own interpretations of the stimuli words. On the other hand, they could be evaluating how well the information provided in the definitions compensated for their lack of knowledge of the meaning of the stimuli words. It could also be the case that the choice between the two interpretations varied across participants, or across items. Although the procedure of this survey did not have a separate measure of whether or not the participants were able to derive their own 'a priori' interpretation, the observed results suggest that, at least for less transparent types, the participants appeared to lack the sufficient prior knowledge, and therefore had to rely upon the definition. This can explain higher evaluations of W1W2 definitions of AD and AC formations. The present results support the claim in Gries (2006) that recognisability of the source words can be used as a criterion of well-formedness of blends, which helps to distinguish them from clipping compounds. The most apparent difference between evaluations of blends that was revealed in this study is that blends with high degree of formal transparency, i.e. blends which preserve at least one of their source words in full, are evaluated differently from those with low formal transparency (AD blends like blizzaster). Clipping compounds (or AC forms, such as rumint $\leftarrow$ rumour + intelligence), behave similarly to low transparency AD blends. It is worth noting that clipping compounds seem to be more sensitive to the presence of the source words in the 132 
definition than even low transparency blends. Although this tendency did not reach the level of statistical significance to be revealed in the decision tree or regression analysis, the overall distribution of the responses by blend type (Figure 13) suggests that clipping compounds are given lower evaluations than any other target words in the sample. These observations indicate that clipping compounds may be processed differently from all types of blends, including lower transparency AD forms. Judging by the low marks words like rumint get, people have difficulty understanding them, and the only thing that seems to be able to save the situation is the presence of both their source words in the definition. However, further study is needed to investigate whether AC forms are significantly different from $\mathrm{AD}$ forms in this respect.

This research is, to the best of my knowledge, the first study of English blends which 1) attempts to evaluate readers' understanding of blends by analysing experimentally induced responses, 2) compares the participants' evaluation of blends and clipping compounds. Moreover, the study focusses entirely on novel blends, which distinguishes it from a number of earlier works in the field. On the one hand, this combination of new materials and new methods can help look at blending from a new angle and, perhaps, find 'a flash of insight' Bauer (2012) hoped for. On the other hand, it means choosing a path 'less traveled by', with a lot of bumps on the way, and possibly a dead end. Among the factors which may be regarded as 'bumps' in that they may have caused difficulty in interpreting the results is, for example, the form of the experiment that was used, that is, a questionnaire. Such a method implies using a subjective measure of the plausibility of the definition, which can depend on a variety of factors, both linguistic and extralinguistic, and only a small number of those (in this case, a few demographic characteristics of the participants and formal and semantic characteristics of target words) can be formalised for statistical analysis. When questionnaire responses form the dependent variable for analysis, a great deal may depend on how the questions were interpreted by the participants, and whether they were interpreted in the same way as the researcher expected them to be. In this study there is no guarantee that the responses that were produced were indeed the evaluations of the definitions, rather than of the target words themselves. A further study involving online measures and reducing the possibility of strategic and/or conscious answers on the part of the participants is needed to further investigate the relationships between the source words' recognisability and recoverability, on the one hand, and the processing of the blends and clipping compounds, on the other hand. Another factor which may have decreased the interpretability of the 
results is the high degree of variance of the lexical material (various structural types of the target words, differences in their semantics, length, frequency of the source words, etc.). It is hardly possible to find a satisfying number of blends and clipping compounds which would be matched for length, frequency, etc. The results of this research, however, can help select those characteristics of target words which are important to control because they appear to influence the participants' responses to them. For instance, as the structural type appeared to be an important predictor of the response, I propose restricting future study to fewer structural types in order to be able to make reliable comparisons between them. It may be the case that some information will be not available if we are not testing the full range of structural types which can be found, but concentrating on fewer distinctly different types of target words can help provide more well-supported explanations of their processing which can afterwards be tested on more structural types.

Despite the drawbacks outlined above, the results of this survey provide some clues about the importance of the different formal properties of the target words, as well as their understanding. The observed results can be regarded as further evidence, in addition to those reported, e.g. in Gries (2012) and in Chapter 4 of the present thesis, that the process of blending results in formations consisting of the beginning of one word and the ending of another (with a possible overlap), which are different from clipping compounds (combining the beginnings of two or more words) not only in terms of form, but also in terms of the relationships between the formation and its source words. The results of the survey also imply that the relationship between the structure of the blend and its plausibility from the reader's point of view is not straightforward, and it is possible that different cognitive factors are involved in the perception and understanding of blends of different structural types and of clipping compounds. To get a closer look at the perception of blends and clipping compounds, I carried out a psycholinguistic experiment which is discussed in the following chapter. On the one hand, the methods used for the next stage of this research allow us to look at the recognition of the source words in a more straightforward way than the questionnaire discussed in this chapter, on the other hand, I will henceforward use fewer structural types of target words which, as the above analysis suggests, is more practical for the aims of the study. 


\section{Chapter 7. Can you find an academic in an acatramp? Priming effect of blends and clipping compounds on the processing of their source words}

The study described in this chapter has been inspired not only by the analysis of gaps in the literature on blends and the corpus data analysis, but to a great extent by the results of the web survey discussed in the previous chapter. In particular, it was found that full preservation of the source words in a blend word clearly influences the evaluation of the definition of the blend. It is not so clear, however, whether blend words which do not preserve any of their source words in full (AD) are significantly different from clipping compounds (AC) in terms of the recognition of their source words. Both earlier research and my own analysis of the structural properties of both types of formations suggest there should be differences. Therefore, the next stage of this research is an experimental study in which $\mathrm{AC}$ and $\mathrm{AD}$ forms are compared in terms of the recognition and processing of the W1 and W2 source words contained in those forms. Both groups will be compared to WW blends which fully preserve their source words and are therefore more transparent than both $\mathrm{AD}$ and $\mathrm{AC}$.

Studies of visual word recognition reveal different effects of word properties on lexical decision latencies, as discussed in Chapter 5. The research presented in the current chapter studies the link between formal transparency (i.e. what portion of the original word is preserved in the shortening) and the recoverability of meaning in two types of complex formations: blends and clipping compounds. The results presented in Chapter 6 suggest that WW blends with a higher degree of formal transparency (such as predictionary) may produce stronger priming of their source words in a lexical decision task than either AD blends with a lower degree of formal transparency (e.g. scoratorium) or clipping compounds (e.g. finlit). The experiment involves an unconventional way of presenting primes via an identification and production task which precedes lexical decision task.

\subsection{Background and rationale}

Are blend words processed in the same way as regular and productive formations such as compounds and derivatives? This question has been approached in a number of experiments by Lehrer $(1996,2003)$ and Lehrer and Veres (2010). The researchers tried to find evidence for rapid and automatic decomposition of blends into their source 
words, and, as stated in Chapter 5, did not succeed. Nevertheless, the results of Lehrer's experiments demonstrated evidence of associations between blends and their source words in language users' memories. In particular, presenting a blend in a task which requires participants to identify its source words results (if the words are recognisable from the blend) in activation of their mental representations. This in turn can influence the participants' performance in a subsequent task (in Lehrer and Veres (2010), a stem completion task). If this is the case, then different results should be obtained for different structural types of blends, depending on the recognisability of their source words. In Lehrer and Veres' study, blends of four structural types were used as stimuli for the identification and production task: splinter + word (AW in my notation, e.g. qualatex $\leftarrow$ quality + latex), word + splinter (WD, e.g. beermare $\leftarrow$ beer + nightmare), two splinters (AD, e.g. snizzle $\leftarrow$ snow + drizzle) and complete overlap (WW, e.g. palimony $\leftarrow$ pal + alimony). The difference between the structural types found in the study was, however, not statistically significant (see section 5.2 for details).

For this research I adapted some of the methods used by Lehrer and Veres and used them on a different set of data to study the differences between processing fully overlapping WW blends, non-overlapping AD blends (the 'two splinters' type in Lehrer's terminology) and clipping compounds, or AC forms. Comparing blends with clipping compounds did not form part of Lehrer and Veres' study, so this research is not simply a replication of their experiment. Rather, taking inspiration from their findings, I am trying to build on them and to extend their implications in new directions. Another important difference between my experiments and those of Lehrer and Veres is that I restrict my stimuli to relatively new blends with splinters which are not likely to have developed into productive combining forms (see criteria in section 4.1). Such a restriction on data sampling is essential because it allows us to separate the effects of recognisability of source words from those of lexicalisation (the case of older blends) and of analogy (the case of productive splinters). Choosing the stimuli for the experiment, I avoided including blends whose splinters showed signs of productivity, such as the initial splinter edu- which is a part of edutainment, edupunk, etc., or the final splinter-noia (present in juvenoia, parentnoia, etc.).

The experiment was designed to verify two hypotheses. The first of them concerns the actual recognition of the source words of blends and clipping compounds: 
1) The source words of blends with a higher degree of formal transparency (WW, e.g. predictionary) will be more easily identified than those of blends with a lower degree of formal transparency (AD, e.g. blizzaster), and of clipping compounds (AC, e.g. finlit). It is also hypothesised that because AC forms are less transparent than any blends (including AD), according to the criteria developed in Chapter 4, their source words will be less easily identified than the source words of AD blends.

The second hypothesis concerns the effect of blends or clipping compounds on the processing of their source words. In a masked priming experiment reported by Lehrer $(2003,2007)$, no priming effect of blends on the processing of source words was found and this result was reported as showing no effect of rapid automatic decomposition of blend words into their constituents. Assuming that, indeed, blends "present a processing challenge" (Lehrer, 2003, p. 379), I focussed not on automatic decomposition but on the effect of prior retrieval of the representation of the source words. Thus, it was decided not to use blends or clipping compounds as masked or unmasked primes in the lexical decision task. Rather, the long-term priming effect of the source words retrieved in an identification and production task was considered to be an experimental variable (see details of the procedure in section 7.4).

2) Prior exposure to blends will facilitate recognition of their source words in a lexical decision task. This effect will be greater for blends with a higher degree of formal transparency (WW) than for blends with a lower degree of formal transparency (AD). This is because the source words of higher transparency blends are more likely to be recognised in the identification / production task which will mediate recognition in the subsequent task. For clipping compounds (AC) no priming effect is expected.

The conditions for the long-time priming effect were created by combining an identification and production task and a lexical decision task so that the blends and clipping compounds used in the former could prime their source words presented as stimuli in the latter.

\subsection{Participants}

Native speakers of English were offered gift vouchers for their voluntary participation in this experiment (the information sheet offered to the participants is provided in 
Appendix 7, and an example of the consent form filled out by each participant is given in Appendix 8). Overall, there were 107 participants (37 male and 70 female), recruited among undergraduate students or staff at Victoria University of Wellington. The age of participants ranged between 18 and 45, the mean age being 22 years, SD=5.5 years.

\subsection{Stimuli}

As pointed out above, the objective of the experiment was to compare blends with a high degree of formal transparency to lower transparency blends and to clipping compounds. Therefore, three groups of complex formations with different degrees of formal transparency were contrasted:

- $\quad$ AC forms (clipping compounds), e.g. hydrail $\leftarrow$ hydr(ogen) + rail(way) (low transparency);

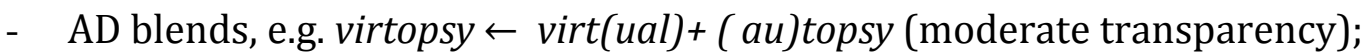

- WW blends (fully overlapping blends), e.g. stoption $\leftarrow$ stop + option (high transparency)

The notation for the structural types of stimuli was used according to the same criteria as in Chapter 6, i.e. only those of $\mathrm{AC}$ and $\mathrm{AD}$ forms which do not preserve either of their source words in full were selected as experimental stimuli because full versus partial preservation can be an important factor influencing word recognition and therefore it is essential to control for it.

In total, 30 words were used for the identification and production task, 10 of each structural type. All the stimuli were selected from the collection discussed in Chapter 4 and therefore attested in media/ corpora no earlier than $1 / 1 / 2000$. None of these words contain productive combining forms registered in dictionaries. However, 6 of 10 clipping compounds contain splinters which appear in other blends or clipping compounds found either in COCA or among those collected for the present research.

The source words of the blends and clipping compounds were then presented as targets in a lexical decision task, 60 target words in total. The same number of pseudowords was also included in the lexical decision task. The pseudowords were created specifically for this experiment, and were matched with the target words in length and also in the degree of orthographic similarity to primes. For example, the blend blizzaster is made of the source words blizzard and disaster so that the letter string blizza-is 
common for the first source word and the blend, and the letter string -aster for the second source word and the blend. The corresponding pseudowords blizzant and colaster have the same letter strings common with the blend blizzaster, and are also matched with the real words blizzard and disaster for the number of syllables. These principles were followed with most of the pseudowords orthographically similar to the real source words of blends and clipping compounds used as stimuli in this experiment. However, the pseudowords similar to the source words of WW blends demanded a slightly different approach. As both the source words are fully preserved in blends like stoption and predictionary, it is impossible to simply retain the relevant part of blend in a pseudoword because it would repeat an existing word, e.g. stop or dictionary. Therefore, pseudowords similar to the source words of WW blends were generated from the corresponding words by replacing some of the letters and/or changing the order of letters in them, e.g. tosp was generated from stop, and doctoilary was generated from dictionary. Thus, such pseudowords had the necessary high degree of orthographic similarity to the source words of blends and at the same time did not look exactly like real words. The parts of the pseudowords which did not repeat the letter strings from real words were either taken from $A R C$ online nonword database (Rastle et al. 2002), or created in such a way that the letter strings resembled those in the database. This approach to generating pseudowords was maintained to make sure that they followed general orthographic and phonological restrictions of English. In addition, the list of experimental stimuli was checked by three native speakers of English, to ensure that they conformed to the phonological and orthographic constraints of English.

\subsection{Procedure}

The experiment consisted of two tasks, completed one after another. Both tasks were created and run in the E-Prime 2.0 software package (Schneider et al. 2002). A response box with NO, OK, and YES buttons was used to input answers for the two tasks. The participants were told that they were going to do two tasks, and that they should use the OK button only for the first task, and YES and NO buttons for the second task.

\subsubsection{Task 1: An identification and production task}

The stimuli were arranged into two lists, with 15 words in each ( 5 AD blends, 5 WW blends, and 5 clipping compounds per list), in order to create a priming condition for the lexical decision task (see section 7.4.2 for details). The participants were randomly assigned to two groups (Group 1 and Group 2), so that one Group 1 was shown one list 
of 15 target words, and Group 2 was shown the other list. All the words were lower case, typed in Courier New font, 13 font size, in light silver colour on black background. The stimuli were presented on a 20 -inch computer screen with the screen resolution of 640 by 480 pixels, the participants were sitting at 50-60 $\mathrm{cm}$ from the screen during the experiment sessions. Each word appeared on the screen for a maximum of $15000 \mathrm{~ms}$.

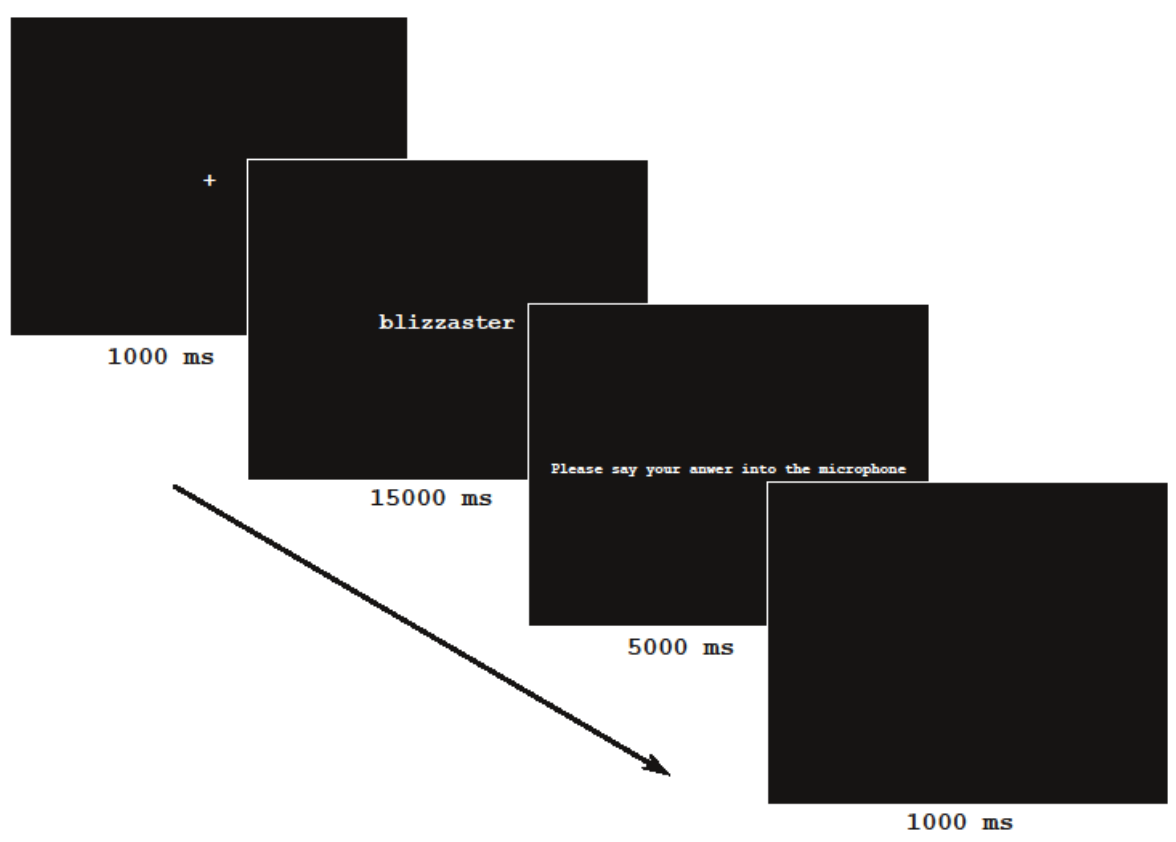

Figure 20. The experimental procedure: Task 1

The participants were instructed to press the OK button as soon as they guessed which two words made up the word on the screen, and then to say these two words into the microphone. As soon as the OK button was pressed, the blend or clipping compound disappeared from the screen, and the instruction 'Please say your answer into the microphone' was presented for $5000 \mathrm{~ms}$ while the participant's response was recorded. The duration of all the responses was less than $5000 \mathrm{~ms}$, and therefore all the responses were fully recorded. After $5000 \mathrm{~ms}$, there was a $1000 \mathrm{~ms}$ blank screen followed by the next fixation cross (Figure 20). A training session with 2 AD blends, 2 WW blends and 2 AC forms preceded the actual task so that the participants could get accustomed to the task. After the training session, participants were given an opportunity to ask questions about the procedure. In addition to the training session, two filler blend stimuli were selected from the same collection of blends as the target words and added to the beginning of the main test block, as a warm-up after the break that followed the training items. The responses to the filler stimuli were not recorded or analysed. The stimuli 
were presented in a pseudo-randomised order, so that no more than two words of the same structural type followed one another.

\subsubsection{Task 2: A lexical decision task}

On completing the first task, the participants saw the instructions for the lexical decision task. They were told that they would see a series of word stimuli, and they were instructed that for each stimulus they should press the YES button if they thought that what they saw was an existing English word, or the NO button if they thought that it was not an existing English word. The YES button was to be pressed with the index finger of the right hand (if the participant was right-handed) and the NO button with the index finger of the left hand. If a participant was left-handed, the buttons were reversed. In this task, unlike in Task 1, the participants were asked to answer as quickly as possible. The stimuli appeared on the screen in upper case, typed in size 13 Courier New font, preceded by a fixation cross and followed by a blank screen (see Figure 21). The screen resolution was the same as in Task 1 . The stimulus stayed on the screen for a maximum of $3000 \mathrm{~ms}$, and disappeared as soon as either the YES or NO button was pressed, or after $3000 \mathrm{~ms}$ if no answer was given.

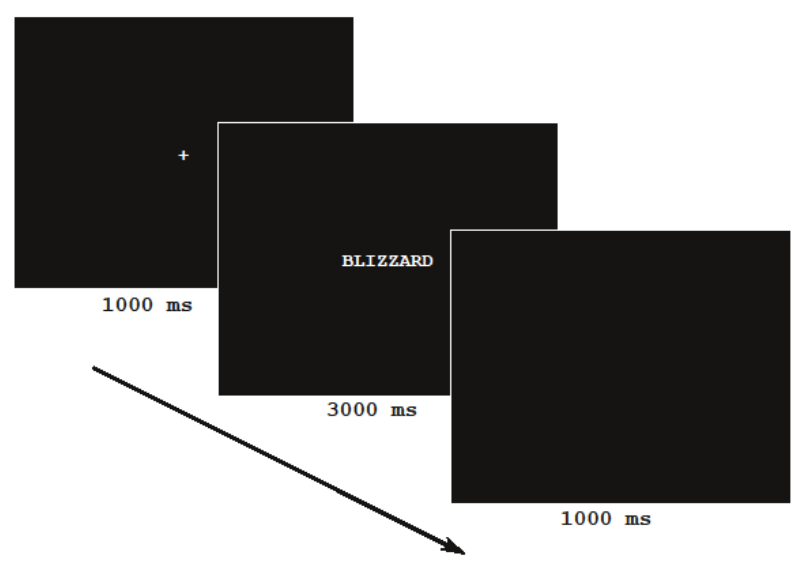

Figure 21. The experimental procedure: Task 2.

In this task, all participants saw source words from each of the total of 30 blend items used in Task 1 . However, because each participant saw only 15 of the blend items in Task 1 (a different 15 depending on whether they were in Group 1 or Group 2 for that task), only half of the target words they saw in Task 2 were source words of items they 
saw in Task 1 (and so could potentially have been recognised in that task), and the other half of target words served as controls. Thus, each target word was shown both to a group of participants who saw the corresponding prime (the blend containing that target as a source word), and to a group of participants who did not. In addition, in Task 2 the target words were arranged in two groups, so that first and second source words of each prime were shown to different participants. As a result, four different combinations of prime and target stimuli were created, as illustrated in Table 17 (the full list of stimuli is presented in Appendix 9). Each group of participants thus saw only one target word per prime, so that there was no possibility that the two source words of an individual blend or clipping compound could prime each other. Nonword stimuli were allocated to groups according to the same principles, and in such a way that word targets and pseudowords orthographically similar to them were shown to different groups of participants. For convenience, the blend words and clipping compounds used as stimuli in Task 1 will be hereafter referred to as primes, and their source words presented in Task 2 as targets.

Table 17. Arrangement of word stimuli into groups according to priming conditions. Group 1 (first two columns) and Group 2 (second two columns) were shown different sets of primes but the same targets; Group A (grey background) and Group B (white background) were shown the same set of primes but different targets

\begin{tabular}{|c|c|c|c|}
\hline \multicolumn{2}{|c|}{ Group 1 Task 1} & \multicolumn{2}{|c|}{ Group 2 Task 1} \\
\hline Group A Task 2 & Group B Task 2 & Group A Task 2 & Group B Task 2 \\
\hline $\begin{array}{l}\text { prime scoratorium - } \\
\text { target } \\
\text { MORATORIUM } \\
\text { (pseudoword } \\
\text { SCORT) }\end{array}$ & $\begin{array}{l}\text { prime scoratorium - } \\
\text { target SCORE } \\
\text { (pseudoword } \\
\text { DROATORIUM) }\end{array}$ & $\begin{array}{l}\text { No prime - target } \\
\text { MORATORIUM } \\
\text { (pseudoword } \\
\text { SCORT) }\end{array}$ & $\begin{array}{l}\text { No prime -target } \\
\text { SCORE } \\
\text { (pseudoword } \\
\text { DROATORIUM) }\end{array}$ \\
\hline $\begin{array}{l}\text { No prime - target } \\
\text { NEGATIVE } \\
\text { (pseudoword } \\
\text { GARTITUDE) }\end{array}$ & $\begin{array}{l}\text { No prime - target } \\
\text { ATTITUDE } \\
\text { (pseudoword } \\
\text { NEGACEDE) }\end{array}$ & $\begin{array}{l}\text { prime negatude- } \\
\text { target NEGATIVE } \\
\text { (pseudoword } \\
\text { GARTITUDE) }\end{array}$ & $\begin{array}{l}\text { prime negatude- } \\
\text { target ATTITUDE } \\
\text { (pseudoword } \\
\text { NEGACEDE) }\end{array}$ \\
\hline $\begin{array}{l}27 \text { participants } \\
\text { in group } 1 \mathrm{~A}\end{array}$ & $\begin{array}{l}27 \text { participants } \\
\text { in group } 1 B\end{array}$ & $\begin{array}{l}27 \text { participants } \\
\text { in group } 2 \mathrm{~A}\end{array}$ & $\begin{array}{l}26 \text { participants } \\
\text { in group } 2 B\end{array}$ \\
\hline
\end{tabular}

As with Task 1, a training session with three word and three nonword stimuli preceded the actual task, to familiarise the participants with the procedure. Participants were given an opportunity to ask questions after the training session. In addition, two fillers (one word, one nonword) served as warm-up items before the main list of targets. 
In sum, the following targets were presented to each of the four groups $(1 \mathrm{~A}, 1 \mathrm{~B}, 2 \mathrm{~A}$ and 2B), arranged in such a way that formal overlap between items seen in a) to h) was minimised:

a) first source words of blends/clipping compounds that were previously shown in Task 1

b) first source words of blends/clipping compounds that were not shown in Task 1

c) second source words of blends/clipping compounds that were previously shown in Task 1

d) second source words of blends/clipping compounds that were not shown in Task 1

e) pseudowords orthographically similar to (a)

f) pseudowords orthographically similar to (b)

g) pseudowords orthographically similar to (c)

h) pseudowords orthographically similar to (d)

\subsection{Methods of data analysis}

\subsubsection{Task 1}

As outlined in section 7.1, it was hypothesised that the constituents of WW blends will be the easiest to identify, AD blends will cause more difficulties, and the constituents of AC forms will be the least recoverable. In accordance with this hypothesis, it is expected that the response choice (and the percentage of correct responses, accordingly) in Task 1 will be different for different target words. In addition to response choice, reaction time was also measured. However, with no time pressure introduced in Task 1, the reaction time analysis was used as supplementary to the analysis of the responses. The percentage of correct answers in naming the first and second source words of all targets in Task 1 was analysed in mixed effects logistic regression models, in which the predictors were the structural type of the target, along with other features of target words (as specified below). The logistic regression models included random intercepts for both item and participant. The random intercepts for items were introduced to account for the fact that the source words of some primes may be more easily recognisable or more difficult to recognise for some reasons which were not already accounted for as fixed item effects. Similarly, random intercepts for participants were introduced to account for the fact that certain participants could recognise more source 
words correctly, or, on the contrary, could give more incorrect answers than other participants. No random slopes for any item or participant effects were included in the logistic models, as adding random slopes did not significantly improve the models.

The mixed effects linear regression analysis of response latencies in Task 1 was run in addition to the logistic regression analysis of the responses. The linear regression models built in the course of this analysis predicted the time it took the participants to give the answer. The reaction time was included in the models as the dependent variable, and various item and participant factors (see the model description in section 7.6.1 for details) as independent variables. The models also included random item and participant factors, and random slope for participant over the length of the primes.

\subsubsection{Task 2}

For Task 2, unlike for Task 1, the primary focus of the analysis was on reaction times: it was hypothesised that response times to word stimuli in Task 2 would be shorter if the relevant primes had previously been shown in Task 1 , and that the priming effect would be stronger for source words of higher transparency blends. Therefore, the structural type of primes shown in Task 1, i.e. AD blends, WW blends, or clipping compounds (AC forms), will be referred to as prime type and will be used as the experimental variable in the data analysis.

To estimate the priming effect, a mixed effects regression analysis of reaction times (RT) was performed. The models included as independent variables both structural type of primes (i.e. AC, AD or WW) and the priming condition (i.e. whether or not the target had a potential prime in the Task 1 stimuli seen by the participant group, and whether or not the prime was correctly identified by participants in Task 1). To adequately interpret the observed values of the dependent variables (that is, reaction time and response choice, in both tasks), a number of other factors had to be accounted for, in addition to experimental conditions. These factors are, on the one hand, participant characteristics, i.e. sex, age and handedness, and, on the other hand, various characteristics of primes and targets. The choice of item variables to be used in the analysis was motivated by the properties of blends observed in Chapter 4, and also by theoretical assumptions from studies on word recognition. Previous studies on visual word recognition reveal the effects of word length (Weekes, 1997), frequency (Grainger, 1990), and, in case of morphologically complex words, family size, or cumulative frequency of all tokens having a particular morpheme (Chialant and Caramazza, 1995). 
Thus, the set of item variables includes frequency of the source words and of the resulting blends/clipping compounds, the length of the splinter, whether the splinter is initial (as is the case for both splinters in AC forms) or final, how similar the blend or clipping compound is to its source words, and how frequently these words co-occur. The full list of item variables is given in Appendix 10, and those variables which are relevant for describing and interpreting the results will be discussed in more detail in the relevant sections. The models in this chapter also include random intercepts for prime and participant, and a nested random effect for Group (to account for the fact that participants of different groups saw different sets of primes). Random slopes for participants over the frequency of the targets, and for item over the age of the participants are also included in the models.

In is essential to check whether the priming effect of the stimuli in Task 1 is due to the recognition of the source words of primes, or simply due to orthographic similarity between primes and targets. One way of looking at this would be to control for orthographic similarity by having a group of control primes with the same degree of orthographic relatedness to targets as the experimental primes. However, such an approach is problematic for the present study for two reasons. Firstly, it would be very difficult (and in some cases not possible at all) to find control words which are as orthographically similar to targets as primes are, and at the same time not morphologically related to targets. Secondly, the identification task which was used to introduce primes could not be used with morphologically simple words. Therefore, in order to look at possible effects of the orthographic similarity between primes and targets, the responses to pseudoword targets were analysed. The regression analysis of pseudoword data was performed in a similar way as the analysis of responses to word targets. The item and participant factors which turned out to have significant effect on the response latencies are different for word and nonword targets, as will be discussed in section 7.6.2. The models for nonword data also included random effects for item and participant, and a random slope for participants over the orthographic similarity between prime and target.

In addition to reaction time analysis, response choices for both words and pseudowords were analysed by means of mixed effect logistic regression modelling (i.e. the dependent variable was the type of response given to each stimulus by each participant, coded as 'correct' or 'incorrect'). Both incorrect and correct responses were used in the analysis 
of response choice, but the reaction time analysis was restricted to correct responses only.

\subsection{Results}

One participant did not give answers to any questions in Task 1, and the same participant and three further participants gave incorrect answers to more than $20 \%$ of the stimuli presented in Task 2. Responses from this total of four participants were excluded from the analysis. The analysis below is therefore based on the responses received from 103 of the original 107 participants: 26 in group 1A, 26 in group 1B, 25 in group $2 \mathrm{~A}$ and 26 in group 2B. In this section I will first present the analysis of Task 1 (7.6.1) and then the analysis of Task 2, the lexical decision task (7.6.2).

\subsubsection{Identification and production task results}

\subsubsection{Identifying SW1 and SW2 of primes}

The percentage of the source words named correctly is different for all three types of stimuli, as shown in Table 18. Correct answers include all cases when the source words were named in exactly the same form as found in the sources, e.g. text and extrovert for textrovert, globalisation and fragmentation for globfrag, and also cases when the same lemma was named, e.g. texting instead of text. Morphologically related, but different lemmas, as globular instead of globalisation were marked as incorrect. This was done not because the clipping compound globfrag cannot possibly mean globular fragment instead of globalisation and fragmentation, according to the original source. The criterion that was used to determine whether the responses were correct or incorrect, was the formal and semantic equivalence between the words named by participants and the actual source words of primes which were subsequently used as targets in the lexical decision task. Despite the fact that globular is both morphologically and semantically related to the target globalisation, it is not possible to conclude they are equivalent. Correct answers for the first and second source words were coded as two separate variables.

As predicted, the source words of WW blends turned out to be the easiest to identify. It is also evident from the table that there is a dramatic difference in the percentage of correct responses between groups, especially for clipping compound stimuli (AC). This might have occurred because the stimuli in Group 1 and Group 2 were not matched for difficulty, or because participants in Group 2 were more capable of recognising the 
source words of the targets for some reason. Nevertheless, the overall tendency is the same in both groups: the source words of WW blends were named correctly by the majority of participants, fewer correct answers were given to AD blends and to clipping compounds. However, it is only in Group 1 that the source words of clipping compounds were the least likely to be named correctly, at least as far as the second source word is concerned.

Table 18. Percentage of correct answers in Task 1

\begin{tabular}{|c|c|c|c|c|c|c|c|c|}
\hline \multirow[t]{2}{*}{$\begin{array}{l}\text { Prime } \\
\text { type }\end{array}$} & \multicolumn{2}{|c|}{$\begin{array}{l}\text { AC } \\
\text { e.g. rumint } \leftarrow \\
\text { rumour }+ \\
\text { intelligence }\end{array}$} & \multicolumn{2}{|c|}{$\begin{array}{l}\text { AD } \\
\text { e.g. blizzaster } \leftarrow \\
\text { blizzard + } \\
\text { disaster }\end{array}$} & \multicolumn{2}{|c|}{$\begin{array}{l}\text { WW } \\
\text { e.g. } \\
\text { predictionary } \leftarrow \\
\text { prediction }+ \\
\text { dictionary }\end{array}$} & \multicolumn{2}{|c|}{ All data } \\
\hline & $\begin{array}{l}\text { Group } \\
1\end{array}$ & Group 2 & $\begin{array}{l}\text { Group } \\
1\end{array}$ & $\begin{array}{l}\text { Group } \\
2\end{array}$ & $\begin{array}{l}\text { Group } \\
1\end{array}$ & $\begin{array}{l}\text { Group } \\
2\end{array}$ & $\begin{array}{l}\text { Group } \\
1\end{array}$ & $\begin{array}{l}\text { Group } \\
2\end{array}$ \\
\hline $\begin{array}{l}\text { SW1 } \\
\text { named } \\
\text { correctly } \\
(\%)\end{array}$ & 17.0 & 53.2 & 45.2 & 72.4 & 78.8 & 81.8 & 47.6 & 68.2 \\
\hline $\begin{array}{l}\text { SW2 } \\
\text { named } \\
\text { correctly } \\
(\%)\end{array}$ & 7.3 & 60.5 & 40.5 & 51.0 & 73.8 & 73.5 & 41.1 & 61.7 \\
\hline
\end{tabular}

The influence of the structural type of primes on the recognition of their source words was estimated in a multiple regression analysis. Two series of logistic regression models were built: the first predicted the identification of the first source word of the primes in Task 1, and the second predicted the identification of the second source word. The participant factors that were taken into consideration were age, sex and handedness. As it could be expected that successful naming of one of the source words triggers naming of the other, a variable indicating correct or incorrect naming of SW2 was included as one of the predictors in models of the error rate for SW1, and vice versa.

A logistic regression model summarised in Table 19 predicts SW1 naming as an outcome of three participant variables, correct / incorrect naming of SW2, and prime type (the full summary of the model is given in (A11.1) in Appendix 11). The model shows significant influence of prime type on the correct naming of SW1. In particular, the difference between AC prime type (used as the reference intercept level) and WW prime type is significant at the $5 \%$ level ( $p=0.0105)$, and the regression coefficient of 2.841 shows that SW1 is more often named correctly if the prime is a WW blend. 
Table 19. The output of the regression model predicting SWI naming

\begin{tabular}{lllll}
\hline $\begin{array}{l}\text { Model formula: } \\
\text { PrimeSW1Correct } \sim \text { Age }+ \text { Sex }+ \text { Handedness + PrimeSW2Correct + PrimeType + (1|Prime) + } \\
\text { (1|Group:uID) }\end{array}$ & \multicolumn{4}{l}{} \\
\hline & Estimate & Std. Error & z value & p value \\
\hline Intercept & -2.179383 & 0.865655 & -2.518 & 0.0118 \\
Age & 0.023592 & 0.010707 & 2.203 & 0.0276 \\
Sexmale & 0.056345 & 0.178936 & 0.315 & 0.7528 \\
Handednessright & -0.004971 & 0.275423 & -0.018 & 0.9856 \\
PrimeSW2Correct & 1.716712 & 0.209339 & 8.201 & 0.0000 \\
PrimeTypeAD & 1.323845 & 1.098240 & 1.205 & 0.2280 \\
PrimeTypeWW & 2.841471 & 1.109863 & 2.560 & 0.0105 \\
\hline
\end{tabular}

Intercept levels: Sexfemale, Handednessleft, PrimeSW2:incorrect, PrimeTypeAC

In addition to the effect of prime type, the model in Table 19 reveals that older participants give more correct answers (the regression coefficient for Age is 0.024, $\mathrm{p}=0.0276$ ) and also that SW1 is more likely to be named correctly if SW2 is correctly identified (the regression coefficient for PrimeSW2Correct is $1.717, \mathrm{p}<0.0001$ ).

Table 20. The output of the regression model predicting SW2 naming

\begin{tabular}{lllll}
\hline $\begin{array}{l}\text { Model formula: } \\
\text { PrimeSW2Correct } \sim \text { Age }+ \text { Sex + Handedness + PrimeSW1Correct + PrimeType + (1|Prime })+ \\
(1 \mid \text { Group:uID) }\end{array}$ & \multicolumn{5}{l}{ Estimate } & Std. Error & z value & p value \\
\hline & -2.99261 & 0.80181 & -3.732 & 0.00019 \\
\hline Intercept & 0.02335 & 0.01270 & 1.839 & 0.06597 \\
Age & -0.19466 & 0.22005 & -0.885 & 0.37637 \\
Sexmale & 0.50931 & 0.34154 & 1.491 & 0.13590 \\
Handednessright & 1.70486 & 0.21752 & 7.838 & 0.00000 \\
PrimeSW1Correct & 0.72455 & 0.92138 & 0.786 & 0.43165 \\
PrimeTypeAD & 2.21640 & 0.92228 & 2.403 & 0.01625 \\
PrimeTypeWW &
\end{tabular}

Intercept levels: Sexfemale, Handednessleft, PrimeSW1:incorrect, PrimeTypeAC

The effect of prime type on SW2 naming is also significant, as is clear from the output of the regression model summarized in Table 20 (A11.2 in Appendix 11). Moreover, analysing Group1 and Group2 separately reveals the significant difference between AD and AC types as well, but only in Group 1, as shown in Table 21 (A11.3 in Appendix 11). The source words of both $\mathrm{AD}$ and WW primes are more frequently recognised correctly than the source words of AC primes, though the difference between $\mathrm{AD}$ and $\mathrm{AC}$ is smaller than the difference between AC and WW. The effect of correct identification of SW1 is a significant predictor of SW2 naming (the regression coefficient for PrimeSW1Correct is 1.953, p<0.0001). As was hypothesised above, recognising one of the source words of a prime triggers recognising the other. 
Table 21. The output of the regression model predicting SW2 naming in Group 1

\begin{tabular}{|c|c|c|c|c|}
\hline \multicolumn{5}{|c|}{$\begin{array}{l}\text { Model formula: } \\
\text { PrimeSW2Correct } \sim \text { Age }+ \text { Sex }+ \text { Handedness }+ \text { PrimeSW1Correct }+ \text { PrimeType }+(1 \mid \text { Prime })+(1 \mid \text { uID })\end{array}$} \\
\hline & Estimate & Std. Error & z value & p value \\
\hline Intercept & -4.59362 & 0.91402 & -5.026 & 0.0000 \\
\hline Age & 0.01729 & 0.01770 & 0.977 & 0.3286 \\
\hline Sexmale & -0.28517 & 0.26669 & -1.069 & 0.2849 \\
\hline Handednessright & 0.48633 & 0.48676 & 0.999 & 0.3177 \\
\hline PrimeSW1Correct & 1.95259 & 0.30843 & 6.331 & 0.0000 \\
\hline PrimeTypeAD & 2.32260 & 0.89555 & 2.593 & 0.0095 \\
\hline PrimeTypeWW & 3.84687 & 0.90368 & 4.257 & 0.0000 \\
\hline
\end{tabular}

Intercept levels: Sexfemale, Handednessleft, PrimeSW1:incorrect, PrimeTypeAC

So far, the only item factor included in the models as fixed effect is prime type. It is important to consider the effect of prime type in the context of other qualities of the stimuli. For this, more complex regression models were built which included other item factors, i.e. prime length and splinter length, and various frequency and similarity measures. The frequency measures considered as item variables (all log-transformed for the regression analysis) are:

- COCA frequency of targets (i.e. the source words of primes);

- the number of Google hits for prime

- Splinter frequency, i.e. the cumulative frequency of all words in COCA that start or end with the splinter retained in prime (for WW primes this means the cumulative frequency of compounds and derivatives including prime)

- Relative frequency, calculated by dividing the Splinter frequency by the frequency of the corresponding source word of the prime.

The recognition of the source words (targets) can also be related to the amount of their material retained in primes, i.e. the splinter length, and also the similarity between prime and target. As a measure of graphic similarity, the Weber and van Orden algorithm was used. The algorithm, which is described in van Orden (1987, p. 196), calculates graphic similarity between two given words, the number of pairs of adjacent letters shared by both words in the same order, the number of pairs of adjacent letters shared by both words in reverse order, the number of single letters shared by words, the average number of letters in the two words, and the ratio of shorter word to longer word. As was the case with the analysis of the web survey data in Chapter 6, various measures of similarity of the source words and blends were used, and the variable which was included in the models discussed below is the one that was selected from a 
cohort of regression models. In addition to graphic similarity, the distance between the switch point and the Uniqueness Point of each source word, as defined in section 5.3, was included as a measure of recognisability of targets. This term, following Gries (2006), was labelled as Switch Point Distance (SPD1 for left splinter, and SPD2 for right splinter). Participant variables, as well as random intercepts for item and participant, were also included in all the models. Including various random slopes for item and participant variables did not significantly improve the fit of any model, therefore models with simple random effect structure are presented here.

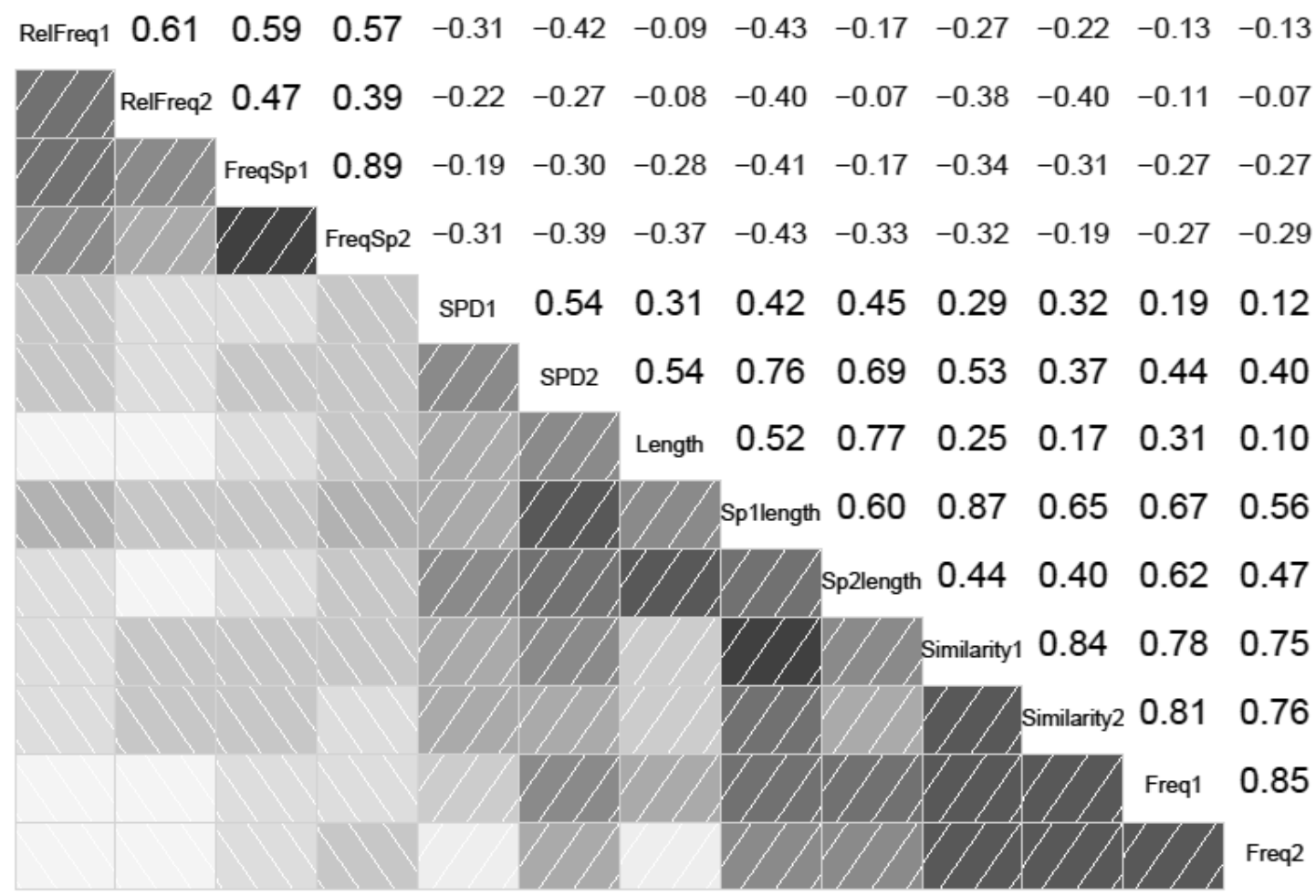

Figure 22. Correlation matrix of a selection of item variables.

The coefficients for the correlation of any two variables in the matrix are shown on the intersection of the vertical and horizontal drawn from the names of the variables in the top right half of the matrix. The deeper the shade of the contra-lateral square in the bottom left half of the matrix, the stronger the correlation

It has to be noted here that some of the item variables correlate with each other, as displayed in Figure 22. In particular, the relative frequency measures (RelFreq1 and RelFreq2) highly correlate with the frequencies of splinters (FreqSp1 and FreqSp2), and raw frequencies of the source words (Freq1 and Freq2) with splinter length (Sp1length and Sp2length). Splinter length also correlates both with SPD (SPD1 for SW1 and SPD2 for SW2) and the Weber and van Orden similarity measure (Similarity1 and Similarity2). 
On the one hand, an analysis of the systematic relations between these various characteristics of blend words is an interesting subject in itself, and looking into these relations might prove sensible, although would need a much larger lexical data sample than the 30 words used for this experiment. On the other hand, multiple correlations shown in Figure 22 are problematic for predicting the effect of these factors on the probability of correct identification of targets, or on the reaction time (see, for example, Baayen (2008) for a detailed discussion of the problem of multicollinearity caused by such situations).

Table 22. Likelihood ratio tests comparing models with splinter frequency and relative frequency predicting SWI naming

\begin{tabular}{lccclccc}
\hline & Df & AIC & BIC & $\begin{array}{l}\text { log- } \\
\text { likelihood }\end{array}$ & $\begin{array}{l}\text { Chi- } \\
\text { square }\end{array}$ & $\begin{array}{l}\text { Df for Chi- } \\
\text { square }\end{array}$ & p-value \\
\hline $\begin{array}{l}\text { model including } \\
\begin{array}{l}\text { splinter frequency } \\
\text { model including } \\
\text { relative frequency }\end{array}\end{array}$ & 9 & 1064.5 & 1112.6 & -523.27 & & & $<0.0001$ \\
\hline
\end{tabular}

One approach to multicollinearity is to select one variable out of a cluster of correlating predictors. This method is appropriate in our case because some of the variables discussed here are different ways of estimating the same property. For example, splinter frequency is built into relative frequency by the way relative frequency is calculated. Such variables as similarity, splinter length and SPD are all related to the degree of preservation of the material of the source words in a blend. Therefore, a series of regression models was built, each of the models including only one variable from each cluster of correlating predictors. The full model predicting the number of correct responses in the identification and production task was built in several steps. Firstly, either splinter frequency (FreqSp) or relative splinter frequency (RelFreq) was selected to account for the splinter frequency effect. The influence of splinter frequency on the identification of source words was better grasped by the relative frequency variables, which is true both for SW1 (as shown in Table 22) and SW2 naming (as shown in Table 23).

Table 23. Likelihood ratio tests comparing models with splinter frequency and relative frequency predicting SW2 naming

\begin{tabular}{lccclccc}
\hline & Df & AIC & BIC & $\begin{array}{l}\text { log- } \\
\text { likelihood }\end{array}$ & $\begin{array}{l}\text { Chi- } \\
\text { square }\end{array}$ & $\begin{array}{l}\text { Df for Chi- } \\
\text { square }\end{array}$ & p-value \\
\hline $\begin{array}{l}\text { model including } \\
\text { splinter frequency } \\
\text { model including } \\
\text { relative frequency }\end{array}$ & 9 & 1232.2 & 1280.3 & -607.09 & & & $<0.0001$ \\
\hline
\end{tabular}


The second step of building the regression models was selecting one variable of the following: SPD, splinter length (Sp1length and Sp2length), Similarity, log transformed source word frequency (Freq1 and Freq2), or PrimeType. Some of these correlating variables (SPD, Sp1length, Sp2length, Similarity, Freq) reflect the degree of preservation of the source words in a blend or a clipping compound. PrimeType, being a nominal variable, is not included into the correlation matrix; however, it is of the same nature as SPD and Similarity variables, and splinter length is built into PrimeType by definition, that is, WW blends are bound to have, on average, longer splinters than AD blends or clipping compounds because the splinter length in case of WW blends is, effectively, the length of the source words. Source word frequency (Freq1 and Freq2) does not directly measure the degree of preservation of the source words, but it correlates with Similarity, splinter length and SPD and therefore is considered one of the competing variables of this cluster. Comparing a series of logistic regression models each of which included one of the above variables showed (see Table 24) that SPD best predicts the identification of SW1.

Table 24. Likelihood ratio tests comparing models with correlating predictors of SW1 naming

\begin{tabular}{|c|c|c|c|c|c|c|c|}
\hline & Df & AIC & BIC & $\begin{array}{l}\text { log- } \\
\text { likelihood }\end{array}$ & $\begin{array}{l}\text { Chi- } \\
\text { square }\end{array}$ & $\begin{array}{l}\text { Df for Chi- } \\
\text { square }\end{array}$ & p-value \\
\hline $\begin{array}{l}\text { model including } \\
\text { SPD }\end{array}$ & 9 & 1059.0 & 1107.1 & -520.51 & & & \\
\hline $\begin{array}{l}\text { model including } \\
\text { splinter length }\end{array}$ & 9 & 1063.3 & 1111.4 & -522.64 & 0.0000 & 0 & 1 \\
\hline $\begin{array}{l}\text { model including } \\
\text { source words } \\
\text { frequency }\end{array}$ & 9 & 1067.4 & 1115.5 & -524.71 & 0.0000 & 0 & 1 \\
\hline $\begin{array}{l}\text { model including } \\
\text { Similarity }\end{array}$ & 9 & 1061.4 & 1109.5 & -521.68 & 1.9286 & 0 & $<0.0001$ \\
\hline $\begin{array}{l}\text { model including } \\
\text { prime type }\end{array}$ & 9 & 1061.8 & 1109.9 & -521.90 & 0.0000 & 0 & 1 \\
\hline
\end{tabular}

Finally, a full model predicting the identification of SW1 was built, including both Relative frequency and SPD, and also prime frequency and prime length. It turned out that the fixed effects of Relative frequency and SPD are highly correlated with one another (the coefficient for the correlation between SPD1 and RelFreq1 is -0.775 , and -0.625 for the correlation between SPD2 and RelFreq2, see model A11.6 in Appendix 11). Therefore, complex terms 'SPD1resid' and 'SPD2resid' were created by residualising SPD of SW1 and SW2 against their relative frequencies, in order to estimate the effect of SPD not already accounted for by relative frequency. Although prime type was not selected as the best predictor of recognisability for a model with simple effects, it was decided to check whether any of the predictors interact with prime type. Adding such an 
interaction was considered because it could be predicted from the way prime types are defined. For example, the relative frequency of WW blends reflects the ratio of the source word frequency to the morphological family frequency, whilst the relative frequency of other primes is, effectively, the ratio of the source word frequency to the cumulative frequency of orthographical neighbours of that word. Adding the interaction between prime type and relative frequency significantly improved the model fit, as shown by a likelihood ratio test (Chi-square $=11.635, \mathrm{Df}=6, \mathrm{p}=0.0406$, see also models A11.7 and A11.8 in Appendix 11). No significant effects of prime frequency and prime length were found, and removing these two predictors from the model did not significantly reduce the model performance (Chi-square $=2.4463, \mathrm{Df}=2, \mathrm{p}=0.2943$ in a likelihood ratio test). Adding random slopes for various participant or item factors did not significantly improve the model, therefore only random intercepts for item and participant and a nested random effect for group were included.

Table 25. The output of the final model predicting SWI identification

\begin{tabular}{|c|c|c|c|c|}
\hline \multicolumn{5}{|c|}{$\begin{array}{l}\text { Model formula: } \\
\text { PrimeSW1Correct } \sim \text { Age }+ \text { Sex }+ \text { Handedness + PrimeSW2Correct }+ \text { RelFreq1 } * \text { PrimeType }+ \text { RelFreq } 2 * \\
\text { PrimeType }+ \text { spd } 1+\operatorname{spd} 2+(1 \mid \text { Prime })+(1 \mid \text { Group:uID })\end{array}$} \\
\hline & Estimate & Std. Error & z value & p value \\
\hline Intercept & -5.02073 & 0.87539 & -5.735 & 0.00000 \\
\hline Age & 0.02369 & 0.01078 & 2.197 & 0.02801 \\
\hline Sexmale & 0.04989 & 0.17900 & 0.279 & 0.78045 \\
\hline Handednessright & -0.01615 & 0.27727 & -0.058 & 0.95355 \\
\hline PrimeSW2Correct & 1.66035 & 0.20674 & 8.031 & 0.00000 \\
\hline RelFreq1 & 6.30828 & 1.95329 & 3.230 & 0.00124 \\
\hline PrimeTypeAD & 0.02347 & 1.41927 & 0.017 & 0.98681 \\
\hline PrimeTypeWW & 5.89311 & 4.46913 & 1.319 & 0.18729 \\
\hline RelFreq2 & 5.44590 & 2.78963 & 1.952 & 0.05092 \\
\hline SPD1resid & -0.84839 & 0.40729 & -2.083 & 0.03725 \\
\hline SPD2resid & -0.10521 & 0.28724 & -0.366 & 0.71414 \\
\hline RelFreq1:PrimeTypeAD & 1.77346 & 2.58348 & 0.686 & 0.49242 \\
\hline RelFreq1:PrimeTypeWW & -7.99432 & 3.24132 & -2.466 & 0.01365 \\
\hline PrimeTypeAD:RelFreq2 & -2.24680 & 3.09976 & -0.725 & 0.46856 \\
\hline PrimeTypeWW:RelFreq2 & -4.38688 & 4.64540 & -0.944 & 0.34499 \\
\hline
\end{tabular}

Intercept levels: Sexfemale, Handednessleft, PrimeSW2:incorrect, PrimeTypeAC,

RelFreq1:PrimeTypeAC, PrimeTypeAC:RelFreq2

The final model in Table 25 (A11.8 in Appendix 11) confirms the effects of participants' age, and of correct identification of SW2, discussed above. The simple effect of prime type on SW1 identification, however, does not reach significance at the 5\% level. The model shows a significant effect of the relative splinter frequency of SW1 (the regression coefficient for RelFreq1 is $6.308, p=0.0012$ ). The higher the frequency of SW1 and the fewer orthographically similar neighbours it has (hence the higher the relative frequency), the higher the probability that SW1 will be correctly named. This effect, 
however, seems to work in an opposite direction for WW blends, as shown by the significant interaction between SW1 relative splinter frequency and prime type, which is visualised in Figure 23.

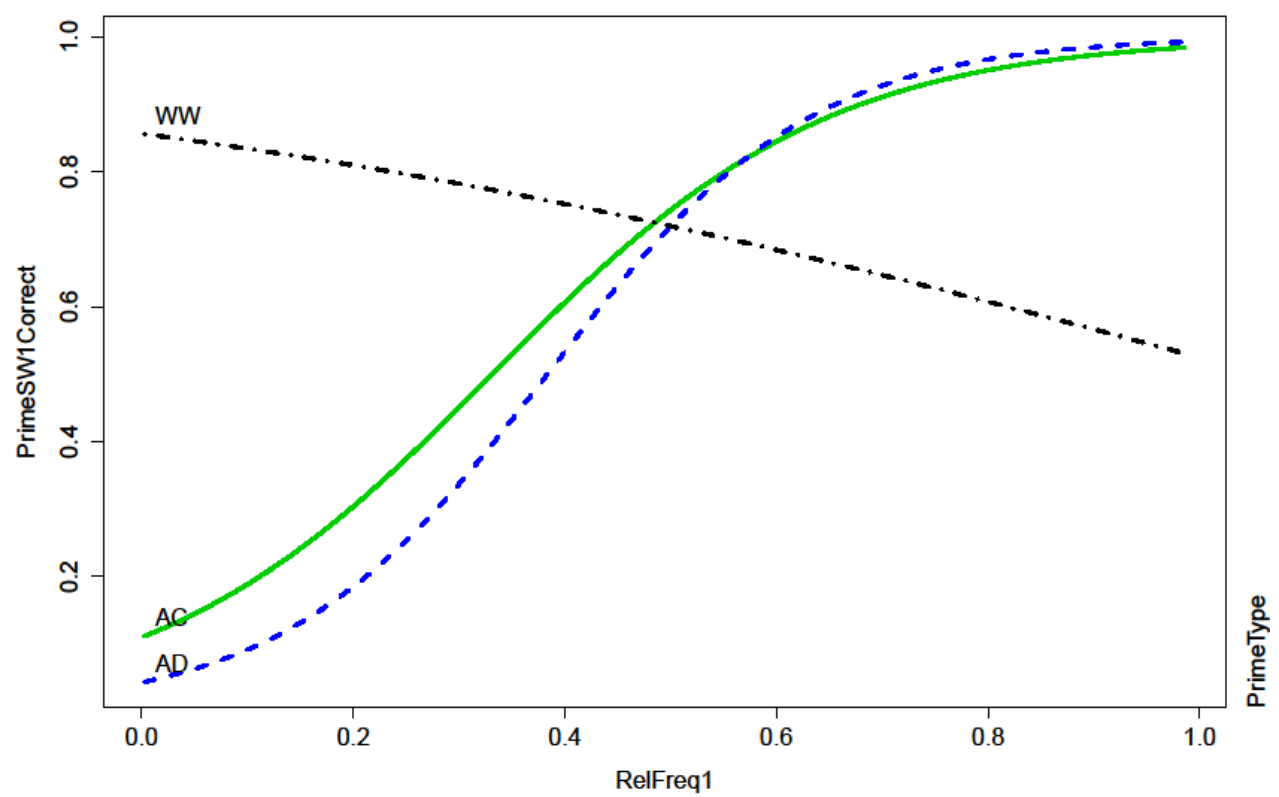

Figure 23.The interaction of prime type and the relative frequency of SWI splinter in the model predicting SW1 naming in Task 1. The vertical axis shows the proportion of correct namings of SW1, the horizontal axis shows the relative splinter frequency of $S W 1$.

Higher relative frequency of SW1 of WW blends, unlike AC or AD forms, results in lower proportion of SW1 named correctly. Such an effect can be explained if we consider again the way relative frequency is calculated. For AC and AD blends high relative frequency of SW1 means that the cumulative frequency of all words attested in COCA that start with the particular splinter is not much higher than the frequency of SW1. This, in turn, implies that SW1 with fewer orthographically similar competitors would be easier to identity by its splinter (e.g. guess that blizza- in blizzaster stands for blizzard). On the other hand, high relative frequency of SW1 of WW blends indicates that there are few compounds or derivatives including their SW1, but it does not rule out cases when there are some high frequency words with similar beginnings, which can inhibit SW1 identification. Therefore, some of the participants named other words beginning with the same letter string as SW1 (e.g. unpacked the WW blend baggravation as bad + aggravation instead of bag + aggravation).

Slightly different results were observed in the logistic regression analysis of SW2 identification, which followed the same steps as for SW1. Firstly, SW2 was correctly 
named less often than SW1, as was earlier shown in Table 18. This is true for all stimuli except AC primes. The participants of Group 2 correctly identified SW2 of AC primes more often than SW1 of those primes (though still not as often as any of the source words of WW primes and, at least in Group 1, AD primes). These results are consistent with earlier findings of the studies of word recognition which show that word beginnings are more important for recognition than word endings (see, for example, Whitney (2001) and White et al. (2008) for experimental findings). Therefore, if the ending of SW2 is preserved in a prime, as is the case for AD blends, SW2 is less easily recognised than SW1. It should be noted that, despite the fact that WW blends fully preserve both SW1 and SW2, SW1 of WW primes was more often named correctly than SW2. This can be explained by the fact that in WW primes the beginning of SW2 does not coincide with the beginning of the whole blend, and therefore may not be recognised as a word beginning at all. In fact, some of the incorrect responses to WW primes (such as stop + motion, or stop + action for the WW blend stoption $\leftarrow$ stop + option) suggest that some of the participants misinterpreted the overlap in WW primes and assumed that only the end of SW2 was preserved in them.

As shown in Table 26, the frequency of the source words turned out a better predictor of SW2 identification than SPD, similarity, splinter length, or prime type. However, adding prime type to the model with source words frequency not only revealed a significant effect of prime type, but also improved the model fit (Chi square $=12.001, \mathrm{Df}=2, \mathrm{p}=0.0025$ for comparing the model including source word frequency only, with the model including also prime type). As a result, the final model predicting SW2 identification (Table 27, the full summary is provided in A11.9 in Appendix 11) included both the frequency of the source words, and prime type.

Table 26. Likelihood ratio tests comparing models with correlating predictors of SW2 naming

\begin{tabular}{|c|c|c|c|c|c|c|c|}
\hline & Df & AIC & BIC & $\begin{array}{l}\text { log- } \\
\text { likelihood }\end{array}$ & $\begin{array}{l}\text { Chi- } \\
\text { square }\end{array}$ & $\begin{array}{l}\text { Df for Chi- } \\
\text { square }\end{array}$ & p-value \\
\hline $\begin{array}{l}\text { model including } \\
\text { SPD }\end{array}$ & 9 & 1237.2 & 1285.3 & -609.62 & & & \\
\hline $\begin{array}{l}\text { model including } \\
\text { Similarity }\end{array}$ & 9 & 1240.1 & 1288.3 & -611.06 & 0.0000 & 0 & 1 \\
\hline $\begin{array}{l}\text { model including } \\
\text { splinter length }\end{array}$ & 9 & 1232.1 & 1280.2 & -607.06 & 5.1222 & 0 & $<0.0001$ \\
\hline $\begin{array}{l}\text { model including } \\
\text { prime type }\end{array}$ & 9 & 1238.1 & 1286.3 & -610.10 & 1.9264 & 0 & $<0.0001$ \\
\hline $\begin{array}{l}\text { model including } \\
\text { source word } \\
\text { frequency }\end{array}$ & 9 & 1233.8 & 1281.9 & -607.91 & 4.3767 & 0 & $<0.0001$ \\
\hline
\end{tabular}


This model does not include sex and handedness, unlike the models above predicting SW1 naming. This is because no significant effect of either factor on SW2 naming was shown, and excluding these predictors from the model did not significantly influence the model fit (Chi square=10.209, $\mathrm{Df}=6, \mathrm{p}=0.1161$ ). Adding other predictors (RelFreq, prime length and prime frequency) and various interactions did not significantly improve the model.

Table 27. The output of the final model predicting SW2 identification

\begin{tabular}{|c|c|c|c|c|}
\hline \multicolumn{4}{|c|}{ Model formula: } & PrimeSW2Correct $\sim$ Age + PrimeSW1Correct + Freq1 + Freq2 + PrimeType $+(1 \mid$ Prime $)+(1 \mid$ Group:uID $)$ \\
\hline & Estimate & Std. Error & z value & p value \\
\hline Intercept & -0.28920 & 0.7698 & -3.757 & 0.000172 \\
\hline Age & 0.02349 & 0.01274 & 1.845 & 0.065108 \\
\hline PrimeSW1Correct & 1.753 & 0.2171 & 8.075 & 0.000000 \\
\hline Freq1 & -0.00056 & 0.00001 & -3.849 & 0.000119 \\
\hline Freq2 & 0.00043 & 0.00001 & 3.367 & 0.000761 \\
\hline PrimeTypeAD & 1.058 & 0.7771 & 1.362 & 0.173322 \\
\hline PrimeTypeWW & 2.854 & 0.7932 & 3.598 & 0.000321 \\
\hline
\end{tabular}

As is the case with SW1 identification, SW2 is more likely to be identified correctly if SW1 is also correctly identified (the regression estimate for PrimeSW1Correct is 1.753, $\mathrm{p}<0.0001$ ). Both SW1 frequency and SW2 frequency have a significant effect on SW2 identification ( $\mathrm{p}<0.001$ for both fixed effects), but they work differently, as shown by the opposite signs of the regression coefficients for the two effects $(-0.00056$ for Freq 1 and 0.00043 for Freq2). It is intuitively clear why higher frequency of SW2 results in it being identified correctly. On the contrary, higher frequency of SW1 appears to inhibit the identification of SW2. It should be also noted that both effects are very small, judging by the extremely low regression coefficients. A possible explanation is that SW1 identification has a stronger influence on SW2 identification than other properties of SW1 or SW2. The model in Table 27 also shows a significant positive effect of WW prime type on SW2 identification (the regression coefficient is 2.854, $\mathrm{p}=0.0003$ ). This means that SW2 of WW primes are more likely to be identified correctly than SW2 of AC primes (as noted above, AC prime type is used as the reference intercept level in all the models). The effect of AD prime type goes in the same direction as the effect for WW prime type (both regression coefficients are positive) but does not reach significance at the $5 \%$ level $(p=0.1733)$.

Even though the effect of prime type is not consistent across all the models discussed above, it is robust enough to assume that the type of prime, i.e. whether it is a high 
formal transparency WW blend, a lower formal transparency AD blend, or a clipping compound (AC), does affect the recognition of the source words of the prime.

\subsubsection{Reaction time in Task 1}

Although Task 1 was not, strictly speaking, a reaction time task, i.e. the participants were not asked to answer as quickly as possible, the analysis of the reaction time was run as supplementary to the source word identification analysis presented above. This was done in order to estimate whether the factors which influence the probability of correct naming of the source words of primes also affected the response latency. A multiple regression analysis was performed, with reaction time (RT) as dependent variable, and the item and participant factors discussed in section 7.6.1.1 as independent variables.

The factors that have significant influence on the reaction time to stimuli in Task 1 are included in the model summarised in Table 28 (the full summary is provided in A11.10 in Appendix 11). Apart from random effects of prime and participant, this model includes a random slope for prime length across participants, which was found to significantly improve the model fit. The increase of prime length indicates two features of primes which could influence the participants' response latencies in different ways. On the one hand, longer primes take longer to read, and therefore the random slope for prime length across participants would reflect individual differences in reading speed. On the other hand, longer primes may contain more material of their source words (WW blends, which are more formally transparent than other primes, are also longer) and therefore be easier to unpack.

Table 28. The output of the model predicting RT in Task 1

\begin{tabular}{llll}
\hline $\begin{array}{l}\text { Model formula: } \\
\text { Stimulus1.RT } \sim \text { PrimeLength + PrimeSW1Correct * PrimeSW2Correct + (1|Prime) }+ \\
\text { (1+PrimeLength|uID) }\end{array}$ & \multicolumn{3}{l}{} \\
\hline & Estimate & Std. Error & t value \\
\hline Intercept & 5819.7 & 1003.6 & 5.799 \\
PrimeLength & 304.2 & 110.6 & 2.751 \\
PrimeSW1Correct & -3311.7 & 278.8 & -11.877 \\
PrimeSW2Correct & -2657.0 & 326.1 & -8.147 \\
PrimeSW1Correct:PrimeSW2Correct & 1206.8 & 389.2 & 3.101 \\
\hline Intercept & & &
\end{tabular}

Intercept levels: PrimeSW1:incorrect, PrimeSW2: incorrect

Including random slopes in a regression model makes it impossible to calculate the $\mathrm{p}$ values estimating the significance of the effects in the model. A way of assessing significance at the $5 \%$ level which is suitable in this case is "to check whether the absolute value of the t-statistic exceeds 2" (Baayen, 2008, p. 270). This method of assessing significance will henceforward be used for all models with random slopes. 
The model in Table 28 shows that it takes longer to identify the source words in longer primes (hence the positive value of the regression coefficient for prime length). The model also includes negative separate effects of the correct identification of SW1 and SW2 on the RT, which indicate that it takes less time for the participants to give their answer if they correctly identify either SW1 or SW2. This is also true for cases when both SW1 and SW2 are named correctly, but the positive regression coefficient for the interaction of these two factors shows that these effects are not simply additive. The model shows that the participants who name both SW1 and SW2 correctly tend to answer faster but not as fast one would expect if SW1 and SW2 were acting completely independently of each other, which is most likely a floor effect.

As is clear from the analysis above, although the structural type of prime appears to influence the identification of targets, it does not affect the speed of response. The following section discusses whether the type of prime influences the recognition of targets in a subsequent lexical decision task.

\subsubsection{Lexical decision task results}

As mentioned in section 7.5, the primary focus of the analysis of the lexical decision task is on the reaction time. An analysis of errors will also be presented in addition to the RT analysis.

\subsubsection{Reaction time analysis}

The RT analysis is based on responses from 103 participants whose overall error rate in Task 2 is smaller than 20\%. Incorrect answers (i.e. pressing the NO button for word stimuli or YES answers to pseudoword stimuli, making up $4.5 \%$ of all responses to word stimuli and $8.7 \%$ of responses to pseudoword stimuli) are also excluded from the analysis. A lower response threshold of $200 \mathrm{~ms}$ was set, but no responses were faster than this. On the other hand, about $3 \%$ of responses were more than $2.5 S D$ above the overall mean RT. On studying the distribution of RT it was decided, however, to keep the slow responses in the data set. The reason they cannot be simply discarded as outliers is that the responses are not normally distributed, as illustrated in the density plot in Figure 24. The strong right skew of the distribution reflects the large number of slow responses observed in the data. 


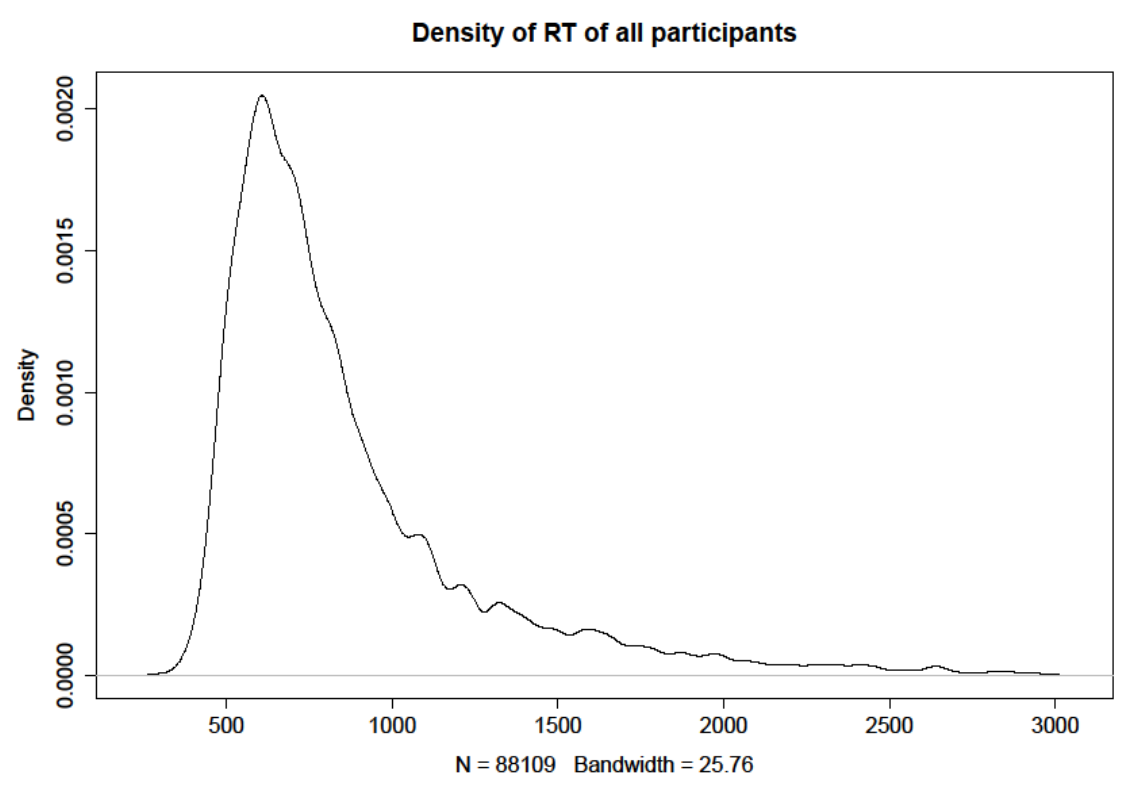

Figure 24. The distribution of RT in Task 2.

In regression analysis, a power transformation function was used to normalise the distribution of the dependent variable, following the method suggested in Weisberg (2005). After transformation, the number of outliers did not exceed $1 \%$ of the data. When regression models were built both for full data and for data with the slowest responses $(0.96 \%)$ excluded, it turned out that reducing the data did not significantly (or even noticeably) change the results. Therefore, all the slow responses were kept in the data set for final modelling. Another consequence of the data transformation is that the regression coefficients in the models below are small (their absolute values are below 0.0001). In order to interpret the output of the models, it is more important to look at the signs of the coefficients and compare the coefficients to each other, rather than concentrate on absolute values. In what follows, I first present the results of a multiple regression analysis of RT to word targets, and then to pseudowords.

\section{Word targets}

Even a casual glance at the descriptive statistics of the RTs makes it evident that the reaction time is different across priming conditions (Table 29). In particular, the source words of AD or WW primes are recognised faster in Task 2 if they were named correctly in Task 1. This is not true, however, of targets which are the source words of AC primes. On the contrary, the mean reaction to such targets is slower in both priming conditions (744 ms if target was named correctly, and 749 if prime was shown but target was not named) than if no prime was shown (726 ms). 
Table 29. Mean reaction time to target words in Task 2 for different priming conditions, millisecs (SD)

\begin{tabular}{llll}
\hline Priming condition & $\begin{array}{l}\text { Prime shown in } \\
\text { Task 1, and target } \\
\text { named correctly }\end{array}$ & $\begin{array}{l}\text { Prime shown in } \\
\text { Task 1, and target } \\
\text { not named } \\
\text { correctly }\end{array}$ & No prime \\
\hline AC prime & $744(292)$ & $749(275)$ & $726(260)$ \\
AD prime & $682(244)$ & $703(271)$ & $705(278)$ \\
WW prime & $683(235)$ & $703(237)$ & $726(267)$ \\
All words & $704(260)$ & $718(262)$ & $719(269)$ \\
\hline
\end{tabular}

The effect of both prime type and priming condition (i.e. whether the prime was shown in Task 1 or not, and whether the target was named) on the response latency in the lexical decision task was explored in multiple regression analysis. The regression models presented below include the same participant variables as the models in section 7.6.1. The item variables used for the analysis were selected and labelled in a different way, accounting for the fact that two targets can share the same prime. The following item variables were used for the regression analysis (the full list of abbreviations for item variables is given in Appendix 10):

- $\quad \log$ transformed Google frequency of prime (LogPfreq);

- log transformed COCA frequency of the target (LogTfreq);

- relative splinter frequency of the corresponding splinter of prime (Prelfreq), calculated as the cumulative frequency of words beginning or ending with the splinter divided by the frequency of the target, using the method suggested in Cook and Stevenson (2007);

- similarity between target and prime (Weber and van Orden (1987) spelling distance between target and prime, labelled as Similarity);

- SPD between prime and target (Pspd):

- prime length (Plength);

- target length (Tlength);

- Splinter length (SpLength);

- prime type (AC, AD or WW)

The effect of the exposure to primes in Task 1 on the response latency is revealed in the regression model displayed in Table 30 (the full summary of the model is given in A11.11 in Appendix 11). Judging by the negative regression coefficient (-0.00001079) for PrimeShownTRUE variable, RT in Task 2 decreases (i.e. the recognition of targets is facilitated) if the prime was shown in Task 1. On the other hand, no significant effect of 
correct naming of SW1 or SW2 is revealed as shown by high p-values for PrimeSW1CorrectTRUE and PrimeSW2resid. It has to be noted that the latter variable accounts for the effect of SW2 naming not already accounted for by SW1 naming (i.e. SW2 naming residualised against SW1 naming). This variable was introduced because of high correlation between PrimeSW1Correct and PrimeSW2Correct variables $(r=0.519)$ which was also revealed in Task 1 analysis in section 7.6.1. The regression analysis was carried out with either PrimeSW1Correct and PrimeSW2resid variables, or with PrimeSW2Correct and PrimeSW1resid variables (i.e. when SW1 naming was residualised against SW2 naming). As the outputs of the models are very similar in both cases, only the former combination of variables is discussed here. Other simple item effects revealed in the model in Table 30 are those of target length and target frequency. Longer targets take more time to be recognised than shorter ones, and more frequent targets are recognised faster (the regression coefficients for Tlength and LogTfreq are negative). All these effects are highly significant ( $\mathrm{p}<0.0001$ for each).

Table 30. The output of the model predicting RT to words in Task 2, with simple effects only

\section{Model formula:}

bcPower(exp.words.noerr\$Stimulus2.RT, pt\$roundlam) $\sim$ Sex + LogTfreq + PrimeType + PrShown + Tlength + PrimeSW1Correct + PrimeSW2resid + (1| uID) + (1| Target $)$

\begin{tabular}{lllll}
\hline & Estimate & Std. Error & t value & p value \\
\hline Intercept & $8.881 \mathrm{e}-01$ & $5.250-05$ & 16918.304 & 0.00000 \\
Sexmale & $1.112 \mathrm{e}-04$ & $2.040 \mathrm{e}-05$ & 5.454 & 0.00000 \\
LogTfreq & $-2.313 \mathrm{e}-05$ & $3.966 \mathrm{e}-06$ & -5.832 & 0.00000 \\
PrimeTypeAD & $-1.628 \mathrm{e}-05$ & $1.695 \mathrm{e}-05$ & -0.960 & 0.341 \\
PrimeTypeWW & $-8.008 \mathrm{e}-06$ & $1.856 \mathrm{e}-05$ & -0.431 & 0.668 \\
PrimeShownTRUE & $-1.079 \mathrm{e}-05$ & $1.171 \mathrm{e}-06$ & -9.210 & 0.00000 \\
Tlength & $1.696 \mathrm{e}-05$ & $3.157 \mathrm{e}-06$ & 5.370 & 0.00000 \\
PrimeSW1CorrectTRUE & $-1.623 \mathrm{e}-09$ & $1.465 \mathrm{e}-06$ & -0.001 & 0.999 \\
PrimeSW2resid & $-3.639 \mathrm{e}-08$ & $1.473 \mathrm{e}-06$ & -0.025 & 0.980 \\
\hline
\end{tabular}

Intercept levels: Sexfemale, PrimeTypeAC, PrimeShownFALSE, PrimeSW1CorrectFALSE

The effect of the correct naming of SW1 and SW2 is not significant in the model above, but this may be due to the fact that the data for this model included cases when the corresponding prime was not shown at all. If a prime was not shown it makes no sense to consider the effect of these two variables on the response latencies. Therefore, a regression model similar to the one in Table 30 was built to analyse the reaction times only to those targets which could potentially be recognised in Task 1 (that is, only if prime was shown). The summary of the model in Table 31 shows that even for this subset of data no significant effect of SW1 or SW2 naming is revealed. Adding the interaction of these two variables with prime type did not significantly improve the model fit (Chi square $=2.1946, \mathrm{df}=4, \mathrm{p}=0.7$ ), nor did it reveal any significant effect of the correct naming of the source words. Therefore, in what follows only the models 
predicting the reaction time for the full data set (that is, including cases when primes were not shown) will be discussed.

Table 31. The output of the model predicting RT to words in Task 2, for PrimeShownTRUE condition

\begin{tabular}{|c|c|c|c|c|}
\hline $\begin{array}{l}\text { Model formula: } \\
\text { bcPower(words.prime\$ } \\
\text { PrimeSW1Correct + Prin }\end{array}$ & $\begin{array}{l}\text { undlam }) \sim \\
\text { uID })+(1 \mid \text { t }\end{array}$ & LogTfreq & th + Prir & \\
\hline & Estimate & Std. Error & t value & p value \\
\hline Intercept & $8.882 \mathrm{e}-01$ & $5.235-05$ & 16964.852 & 0.00000 \\
\hline Sexmale & $1.171 \mathrm{e}-04$ & $2.185 \mathrm{e}-05$ & 5.358 & 0.00000 \\
\hline LogTfreq & $-2.518 \mathrm{e}-05$ & $3.132 \mathrm{e}-06$ & -6.439 & 0.00000 \\
\hline Tlength & $1.504 \mathrm{e}-05$ & $3.157 \mathrm{e}-06$ & 5.370 & 0.00000 \\
\hline PrimeTypeAD & $-2.861 \mathrm{e}-05$ & $1.660 \mathrm{e}-05$ & -1.723 & 0.0909 \\
\hline PrimeTypeWW & $-3.222 \mathrm{e}-06$ & $1.818 \mathrm{e}-05$ & -1.773 & 0.0821 \\
\hline PrimeSW1CorrectTRUE & $1.577 \mathrm{e}-09$ & $2.021 \mathrm{e}-06$ & -0.008 & 0.994 \\
\hline PrimeSW2resid & $-7.232 \mathrm{e}-08$ & $2.033 \mathrm{e}-06$ & -0.036 & 0.972 \\
\hline
\end{tabular}

The models above include simple effects only, no interactions and no random slopes for item or participant variables, but have random intercepts for item and participant. No significant effects of other item variables, that is, prime length, splinter length, Similarity, relative splinter frequency, SPD and prime type, were found. These predictors were introduced one by one, rather than added all to one model because they correlate highly with each other, as is clear from Figure 25. It is not surprising because they are, effectively, the same variables as in Task 1, labelled in a different way.

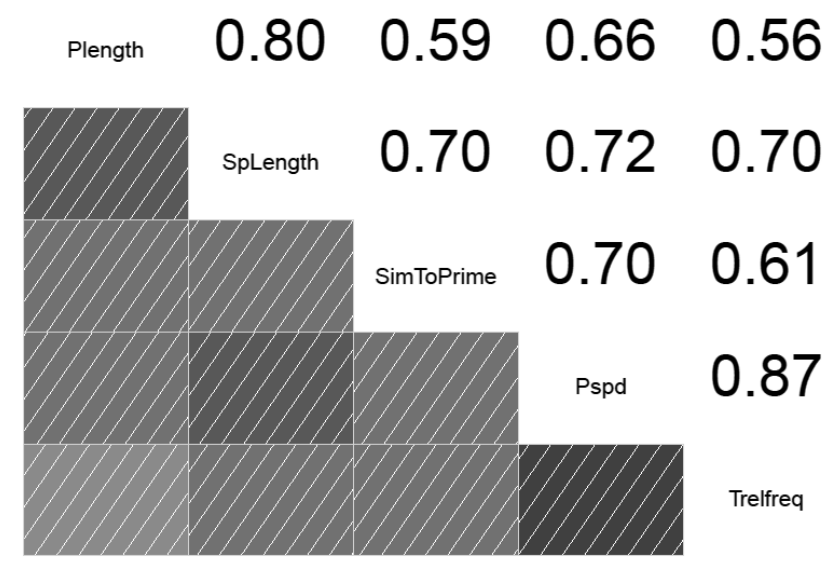

Figure 25. Correlation matrix of a selection of target variables.

The coefficients for the correlation are shown on the intersection of the vertical and horizontal drawn from the names of the variables in the top right half of the matrix. The deeper the shade of the contralateral square in the bottom left half of the matrix, the stronger the correlation

Although the simple effect of prime type did not prove to be significant in the models above, the data in Table 29 suggest that there is a difference in the priming effect for the source words of primes of different structural types. A possible explanation would be the interaction between the effect of priming condition and prime type. Therefore, a 162 
more complex model was built, including the significant simple effects from the model in Table 30, and also the interaction of PrimeShown and PrimeType variables.

Table 32. Likelihood ratio tests comparing models with increasingly complex structure

\begin{tabular}{|c|c|c|c|c|c|c|c|}
\hline & Df & AIC & BIC & $\begin{array}{l}\text { log- } \\
\text { likelihood }\end{array}$ & $\begin{array}{l}\text { Chi- } \\
\text { square }\end{array}$ & $\begin{array}{l}\text { Df for Chi- } \\
\text { square }\end{array}$ & p-value \\
\hline $\begin{array}{l}\text { model with simple } \\
\text { effects only }\end{array}$ & 8 & -646055 & -646985 & 323535 & & & \\
\hline $\begin{array}{l}\text { model including } \\
\text { the interaction of } \\
\text { prime type and } \\
\text { priming condition }\end{array}$ & 12 & -647231 & -647127 & 323627 & 184.34 & 4 & $<0.0001$ \\
\hline $\begin{array}{l}\text { model including } \\
\text { variable } \\
\text { interaction, and } \\
\text { random slope for } \\
\text { target frequency }\end{array}$ & 14 & -648553 & -648431 & 324290 & 1325.56 & 2 & $<0.0001$ \\
\hline $\begin{array}{l}\text { model including } \\
\text { variable } \\
\text { interaction, and } \\
\text { random slopes for } \\
\text { target frequency } \\
\text { and Age }\end{array}$ & 16 & -650141 & -650003 & 325087 & 1592.96 & 2 & $<0.0001$ \\
\hline
\end{tabular}

The results of an ANOVA comparison summarised in Table 32 show that the model with interaction outperforms the model with simple effects only (Chi-square $=184.34, \mathrm{df}=4$, $\mathrm{p}<0.0001)$. In addition to that, various random slopes for item and participant variables were added to the model. The random slopes for target frequency across participants and for the age of participants across the different items significantly improved the model fit. The final model is summarised in Table 33 (the full summary of the model is provided in A11.13 in Appendix 11).

Table 33. The output of the final model predicting RT to words in Task 2

\section{Model formula:}

bcPower(exp.words.noerr\$Stimulus2.RT, pt\$roundlam) PrimeType * PrShown + Sex + LogTfreq + Tlength + (1+LogTfreq|uID) + (1+Age|Target)

\begin{tabular}{lllll}
\hline & Estimate & Std. Error & t value & p value \\
\hline Intercept & $8.881 \mathrm{e}-01$ & $5.318 \mathrm{e}-05$ & 16700.262 & 0.00000 \\
PrimeTypeAD & $-7.910 \mathrm{e}-06$ & $1.724 \mathrm{e}-05$ & -0.459 & 0.64812 \\
PrimeTypeWW & $1.049 \mathrm{e}-05$ & $1.886 \mathrm{e}-05$ & 0.556 & 0.58031 \\
PrimeShownTRUE & $6.369 \mathrm{e}-06$ & $1.975 \mathrm{e}-06$ & 3.225 & 0.00126 \\
Sexmale & $1.118 \mathrm{e}-04$ & $2.043 \mathrm{e}-05$ & 5.473 & 0.00000 \\
LogTfreq & $-2.376 \mathrm{e}-05$ & $4.019 \mathrm{e}-06$ & -5.912 & 0.00000 \\
Tlength & $1.664 \mathrm{e}-05$ & $3.199 \mathrm{e}-06$ & 5.202 & 0.00000 \\
PrimeTypeAD:PrimeShownTRUE & $-1.726 \mathrm{e}-05$ & $2.804 \mathrm{e}-06$ & -6.156 & 0.00000 \\
PrimeTypeWW:PrimeShownTRUE & $-3.762 \mathrm{e}-05$ & $2.823 \mathrm{e}-06$ & -13.325 & 0.00000 \\
\hline Intercept levels: PrimeTypeAC, PrimeShownFALSE, Sexfemale, PrimeTypeAC:PrimeShownFALSE
\end{tabular}

Intercept levels: PrimeTypeAC, PrimeShownFALSE, Sexfemale, PrimeTypeAC:PrimeShownFALSE

All the fixed effects that were significant in the model with simple random effects hold in the final model. The simple fixed effects that are included in the final model are those of sex (male participants tend to respond more slowly than females), target length, and target frequency. The response latency increases for longer targets, and decreases with 
the increase of target frequency, which is a common finding in lexical decision experiments, discussed, for example, in Weekes (1997) and Grainger (1990). There is an important difference between the model including simple effects only (Table 30), and the model including the interaction between prime type and priming condition. In the simple model, the priming effect was shown as decreasing the RT for the condition when prime was shown, which is shown by the negative regression coefficient for the variable PrimeShownTRUE in Table 30. In the model with interaction, however, the simple effect of the Priming condition is reversed for AC primes, as illustrated in Figure 26 (the effects are back-transformed for RTs, for the ease of interpretation).

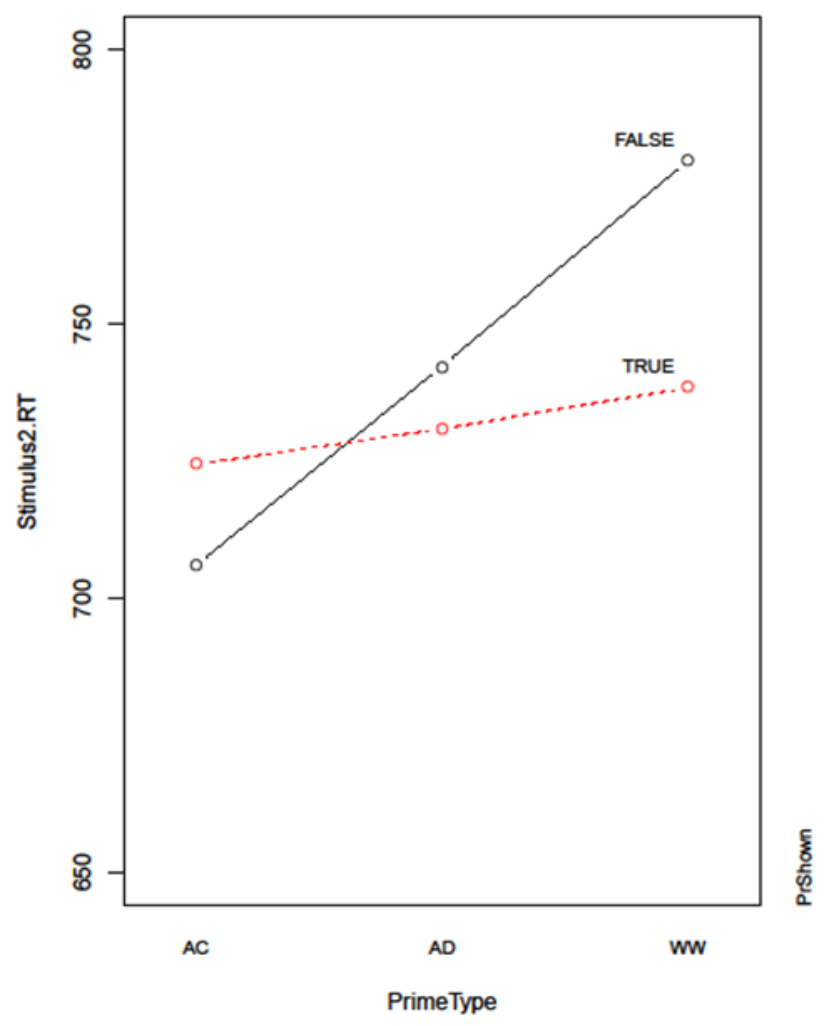

Figure 26. The interaction of prime type and priming condition in the model predicting RT in Task 2. The graph in solid line shows the mean reaction times for different prime types if no prime was shown (FALSE priming condition); the graph in dotted line shows the mean reaction time for different prime types if primes were shown in Task 1 (TRUE priming condition)

The regression coefficients for the Priming effect in Table 33 are easier to understand if we keep in mind that the reference intercept level for prime type in all the models is AC. The positive regression coefficient for PrimeShownTRUE indicates that for AC blends (all the random item and participant effects being accounted for) the RT is larger if the prime was shown in Task 1, in comparison with the No prime condition. The regression coefficients for priming effects for $\mathrm{AD}$ and WW primes, however, have negative signs. Given that the reference level for the interaction term is the targets are the source words 
of AC primes, with no primes shown in Task 1, this means that the response to the source words of $\mathrm{AD}$ and WW primes in Task 2 (if the primes were shown in Task 1 ) is significantly faster than the response to the source words of AC primes for which no primes were shown. Moreover, the model in Table 33 confirms that both AD primes and WW primes are significantly different from AC in this respect $(\mathrm{p}<0.0001$ both for PrimeShownTRUE:AD and PrimeShownTRUE:WW). The partial effects of prime type and priming condition plotted in Figure 26 suggest that the exposure to AD and WW Primes in Task 1 results in faster recognition of Targets in Task 2.

The facilitating effect of exposure to $\mathrm{AD}$ and $\mathrm{WW}$ primes on the recognition of Tagrets is in agreement with Hypothesis 2 in section 7.1. As for AC primes, not only does prior exposure to them not facilitate the recognition of their source words, but it also appears to inhibit their recognition. The nature of this effect may become more evident if we look at the recognition of nonword targets in the same task.

\section{Nonword targets}

Nonword targets created for Task 2 were matched with the word targets for syllabic length and orthographic similarity to primes, as described in section 7.3. The mean reaction time to nonword targets in the lexical decision task (987 $\mathrm{ms}$ ) is higher than to word targets (714 ms). This is consistent with earlier findings of many lexical decision experiments, e.g. (Forster and Davis, 1984) which show that nonwords are generally responded to more slowly than words. Partially this is because the NO button in this experiment was pressed with the nondominant hand, but also because 'non-acceptance' judgements are generally slower than 'acceptance' judgements if the task is to determine if a given letter string is a word (not to judge if it is a nonword).

Table 34. Mean reaction time to nonwords in Task 2, millisecs (SD)

\begin{tabular}{llll}
\hline Priming condition & $\begin{array}{l}\text { Prime shown in } \\
\text { Task 1, and target } \\
\text { word named } \\
\text { correctly }\end{array}$ & $\begin{array}{l}\text { Prime shown in } \\
\text { Task 1, and target } \\
\text { word not named } \\
\text { correctly }\end{array}$ & No prime \\
\hline AC prime & $1014(475)$ & $1022(463)$ & $973(430)$ \\
AD prime & $1016(415)$ & $1010(415)$ & $1012(436)$ \\
WW prime & $934(402)$ & $977(435)$ & $911(391)$ \\
All nonwords & $987(433)$ & $1003(439)$ & $964(421)$ \\
\hline
\end{tabular}

What is interesting about the reaction to nonwords in this study is that the mean reaction time is larger for nonwords orthographically similar to $\mathrm{AC}$ and $\mathrm{WW}$ primes if the participants had seen the primes in Task 1. For nonwords similar to AD primes there 
is almost no difference in response latency across all priming conditions, as shown in Table 34 .

Table 35. Likelihood ratio tests comparing models with increasingly complex structure

\begin{tabular}{|c|c|c|c|c|c|c|c|}
\hline & Df & AIC & BIC & $\begin{array}{l}\text { log- } \\
\text { likelihood }\end{array}$ & $\begin{array}{l}\text { Chi- } \\
\text { square }\end{array}$ & $\begin{array}{l}\text { Df for Chi- } \\
\text { square }\end{array}$ & $\mathrm{p}$-value \\
\hline $\begin{array}{l}\text { model with simple } \\
\text { effects only }\end{array}$ & 11 & -398540 & -398447 & 199281 & & & \\
\hline $\begin{array}{l}\text { model including } \\
\text { the interaction of } \\
\text { prime type and } \\
\text { priming condition }\end{array}$ & 12 & -398565 & -398463 & 199294 & 26.35 & 1 & $<0.0001$ \\
\hline $\begin{array}{l}\text { model including } \\
\text { variable } \\
\text { interaction, and } \\
\text { random slope for } \\
\text { similarity between } \\
\text { target and prime }\end{array}$ & 14 & -400119 & -400000 & 200073 & 1557.91 & 2 & $<0.0001$ \\
\hline $\begin{array}{l}\text { model including } \\
\text { variable } \\
\text { interaction, and } \\
\text { random slopes for } \\
\text { similarity and Age }\end{array}$ & 16 & -400645 & -400509 & 200339 & 530.73 & 2 & $<0.0001$ \\
\hline
\end{tabular}

A multiple regression analysis of factors which influence the reaction to nonword targets included the same participant and item variables as were included for word targets. The only difference is target frequency: given that all nonwords have zero frequency, there was no point in including it as a variable in the regression models.

Table 36. The output of the final model predicting RT to nonwords in Task 2

\begin{tabular}{|c|c|c|c|c|}
\hline \multicolumn{3}{|c|}{$\begin{array}{l}\text { Model formula: } \\
\text { bcPower(exp.nonwords.noerr\$Stimulus2.RT, ptnw\$roundlam) } \sim \text { Sex + } \\
+ \text { spd.nw + }(1+\text { SimToSW|uID) + (1|Target) }\end{array}$} & \multicolumn{2}{|c|}{ PrShown * PrimeType + tlength } \\
\hline & Estimate & Std. Error & t value & p value \\
\hline Intercept & 1.262 & $1.706 \mathrm{e}-04$ & 7397.691 & 0.00000 \\
\hline Sexmale & $9.596 \mathrm{e}-04$ & $2.245 \mathrm{e}-04$ & 4.275 & 0.00004 \\
\hline PrimeShownTRUE & $1.785 \mathrm{e}-04$ & $1.843 \mathrm{e}-05$ & 9.689 & 0.00000 \\
\hline PrimeTypeAD & $2.153 e-04$ & $1.502 \mathrm{e}-04$ & 1.433 & 0.15808 \\
\hline PrimeTypeWW & $2.668 \mathrm{e}-04$ & $1.567 \mathrm{e}-04$ & -1.702 & 0.09471 \\
\hline Tlength & $2.485 \mathrm{e}-04$ & $3.022 \mathrm{e}-05$ & 8.223 & 0.00000 \\
\hline spd.nw & $-9.084 \mathrm{e}-05$ & $4.676 \mathrm{e}-05$ & -1.943 & 0.03790 \\
\hline PrimeTypeAD:PrimeShownTRUE & $1.169 \mathrm{e}-05$ & $2.605 \mathrm{e}-05$ & -4.489 & 0.00000 \\
\hline PrimeTypeWW:PrimeShownTRUE & $9.573 \mathrm{e}-05$ & $2.615 \mathrm{e}-05$ & -3.660 & 0.00025 \\
\hline
\end{tabular}

The regression analysis for nonwords followed the same steps as for words, that is, first a model with simple fixed effects was built (A11.14 in Appendix 11), and then variable interactions and random slopes were added, but only if adding to the complexity of the model was justified. Table 35 summarises the steps of the regression model building, 
and the final model is shown in Table 36 (the full summary of the model is given in A11.14 in Appendix 11).

Some factors appear to influence the response times for decisions made to nonword and word targets in the same way. In particular, male participants react more slowly both to word and nonword targets (hence the positive regression coefficients for Sexmale in the model in Table 36, as well as in Table 33 above). Longer nonword targets take more time to reject as not being words, as indicated by positive regression coefficient for target length in Table 36. The main difference between the models predicting the reaction time to word and nonword targets is in the direction and strength of the effect of PrimeType, in interaction with the priming condition. Responses to nonword targets orthographically similar to AD and WW primes were slower if the primes were shown in Task 1. The main effect for PrimeShownTRUE variable also has a positive regression coefficient. The regression analysis shows that responses to all nonwords were slower if orthographically similar primes were shown in the task preceding the lexical decision. However, the strength of this effect is different for different structural types of primes, which is revealed by the significant effect of the interaction between prime type and priming condition, as shown in Table 36.

Partial effects of prime type and priming condition in the models predicting RT for words and nonwords are displayed in Figure 27. As it is clear from the left panel (which shows the same effects as Figure 26 above), prior exposure to WW or AD primes reduces the reaction time to their source words in the lexical decision task. The facilitative effect is weaker for $\mathrm{AD}$ primes than for $\mathrm{WW}$ primes, and exposure to $\mathrm{AC}$ primes results in slower reactions rather than faster ones. The right panel of the plot demonstrates that prior exposure to primes slows down the reaction to targets, and this is true for all three prime types, although the inhibitory effect of AD primes is smaller than that of $\mathrm{AC}$ and $\mathrm{WW}$ primes. 

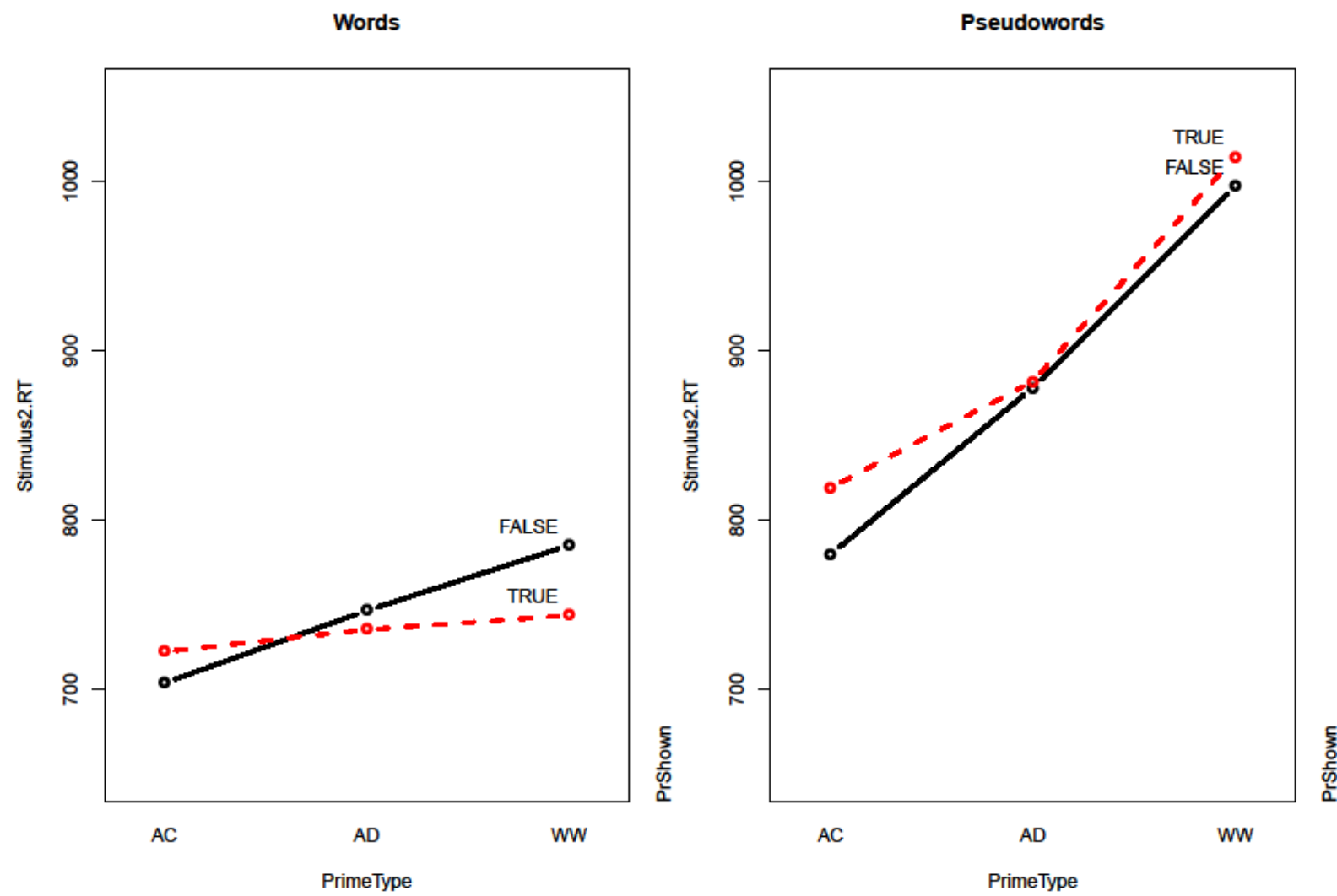

Figure 27. The interaction of prime type and priming condition in the model predicting $R T$ to word targets (left panel) and nonword targets (right panel) in Task 2. The graph in solid line shows the mean reaction times for different prime types if no prime was shown (FALSE priming condition); the graph in dotted line shows the mean reaction time for different prime types if primes were shown in Task 1 (TRUE priming condition)

It appears that the presence of $\mathrm{AC}$ primes has a similar effect on response times to both the source words and to matched nonword targets in this lexical decision task. In both cases, prior exposure to $\mathrm{AC}$ primes results in slower responses to the related targets in Task 2. The analysis of RT to nonwords has also shown similar effect of exposure to AD and WW primes. If a prime was shown in Task 1 , it took the participants longer to recognise an orthographically related nonword. Judging by this result, the priming effect of $\mathrm{AD}$ and WW blends is different for word and nonword targets.

\subsubsection{Error analysis}

The percentage of errors in Task 2 is higher for nonword stimuli than for words, as shown in Table 37. This is an expected result for nonwords, similarly to longer reaction times, in comparison with word stimuli (see 7.6.2.1). For both words and nonwords, the error rate is lower if the prime was shown, but the logistic regression analysis showed that this effect is not significant at the $5 \%$ level. The output of the model predicting the error rate for word targets is displayed in Table 38 (see also the full summary in A11.16 
in Appendix 11), and for nonword targets in Table 39 (see also the full summary in A11.17 in Appendix 11).

Table 37. The proportion of errors in Task 2 for different priming conditions, $\%$.

\begin{tabular}{lllll}
\hline $\begin{array}{l}\text { Priming } \\
\text { condition }\end{array}$ & \multicolumn{2}{c}{ Words } & \multicolumn{2}{c}{ Nonwords } \\
& Prime shown & No prime & Prime shown & No prime \\
\hline AC Prime & 2.43 & 2.82 & 5.35 & 5.98 \\
AD Prime & 4.83 & 4.24 & 9.00 & 11.14 \\
WW Prime & 5.38 & 6.96 & 6.74 & 7.82 \\
Total & 4.22 & 4.68 & 8.52 & 8.72 \\
\hline
\end{tabular}

A significant effect of the participants' age on the error rate is revealed both for words and for nonwords. Older participants made fewer errors in the lexical decision task. For word targets, higher target frequency results in fewer errors. No significant effect of target length on error rate is found for words, but the number of miscategorisations of nonword targets (i.e. YES responses) increases with the increase in their length.

Table 38. The output of the model predicting error rate for words in Task 2

\begin{tabular}{|c|c|c|c|c|}
\hline \multicolumn{5}{|c|}{$\begin{array}{l}\text { Model formula: } \\
\text { Error } \sim \text { Age + LogTfreq + PrShown * PrimeType + (1|uID) + (1|Target })\end{array}$} \\
\hline & Estimate & Std. Error & $\mathrm{z}$ value & p value \\
\hline Intercept & 5.60337 & 2.95716 & 1.895 & 0.05811 \\
\hline Age & -0.13787 & 0.04563 & -3.021 & 0.00252 \\
\hline LogTfreq & -1.26368 & 0.30711 & -4.115 & 0.00003 \\
\hline PrimeShownTRUE & -0.22505 & 0.12365 & -1.820 & 0.06875 \\
\hline PrimeTypeAD & -0.73210 & 1.18797 & -0.616 & 0.53773 \\
\hline PrimeTypeWW & -1.15954 & 1.23062 & -0.942 & 0.34607 \\
\hline PrimeTypeAD:PrimeShownTRUE & 0.49530 & 0.15965 & 3.102 & 0.00192 \\
\hline PrimeTypeWW:PrimeShownTRUE & -0.15126 & 0.15444 & -0.979 & 0.32740 \\
\hline
\end{tabular}

No significant effect of prime type is revealed in the regression modelling. The interaction between prime type and priming condition turned out to significantly improve the model fit for words (Chi-square=20.576, Df=2, $\mathrm{p}<0.0001$ ), but not for nonwords. A positive regression coefficient for PrimeTypeAD:PrShownTRUE: (0.49530) in Table 38 indicates that the source words of the AD primes that were shown in Task 1 were more often misrecognised in Task 2 than the source words of AC primes in no priming condition. It is not clear what this effect is due to, but a possible explanation may be that the recognition of the source words of AD primes in Task 1 interferes with the recognition of the targets in Task 2. 
Table 39. The output of the model predicting error rate for nonwords in Task 2

\begin{tabular}{|c|c|c|c|c|}
\hline \multicolumn{5}{|c|}{$\begin{array}{l}\text { Model formula: } \\
\text { Error } \sim \text { Age }+ \text { Tlength }+ \text { PrShown } * \text { SimToPrime }+(1 \mid \text { uID })+(1 \mid \text { Target })\end{array}$} \\
\hline & Estimate & Std. Error & $\mathrm{z}$ value & p value \\
\hline Intercept & -7.2944155 & 1.5688987 & -4.649 & 0.000003 \\
\hline Age & -0.0597923 & 0.0260627 & -2.294 & 0.021781 \\
\hline Tlength & 0.3428641 & 0.1495786 & 2.292 & 0.021894 \\
\hline PrimeShownTRUE & -0.5218644 & 0.1481934 & -3.522 & 0.000429 \\
\hline SimToPrime & 0.0028572 & 0.0020136 & 1.419 & 0.155926 \\
\hline PrShownTRUE:SimToPrime & 0.0009214 & 0.0002682 & 3.435 & 0.000593 \\
\hline
\end{tabular}

Intercept levels: PrimeShownFALSE, PrimeShownFALSE:SimToPrime $=0$

The recognition of nonwords, on the other hand, is subject to the degree of their orthographic similarity to primes. The interaction of the similarity of the nonword targets to primes and the priming condition is significant, and the model in Table 39 which includes this interaction performs significantly better than the model with no interactions (Chi-square $=14.335, \mathrm{Df}=2, \mathrm{p}<0.0001$ ). Nonword targets are more likely to be erroneously identified as words if orthographically similar primes were seen by the participants in Task 1.

\subsection{Interim conclusions: Recognisability and recognition}

This experiment was designed to study the effect of the formal transparency of blend words and clipping compounds on the recognition of their source words in a lexical decision task. It was hypothesised on the basis of the analysis of the structural properties of blends and clipping compounds (Chapter 4) that the source words of WW blends and AD blends would be recognised more easily than the source words of clipping compounds (AC). Similar assumptions concerning the recognisability of source words were made in earlier studies of blends, e.g. in Gries (2006), on the basis of corpus data analysis. The results of the first part of the experiment (Task 1) confirmed this hypothesis. It was shown that the source words of WW blends are more easily recognised than those of lower transparency AD blends or of clipping compounds. This result is compatible with the findings of earlier experiments by Lehrer (2003) and Lehrer and Veres (2010), which showed that if the source words are fully preserved in blends (which is the case for WW), they are easier to identify. The findings of the present study reveal a statistically significant effect of the structural type of blends on the recognition of their source words, which was not found in earlier experiments. It is also clear from the present results that the source words of AD blends are more easily recognised than the source words of clipping compounds. This comparison was part of the design of my experiment which was not present in the experiments carried out by 
Lehrer and Veres. Although the difference between the recognition of the source words of AD blends and clipping compounds was significant only for Group 1, the overall tendency was observed for both groups.

Easier recognition of the source words of WW blends, in comparison with the source words of $\mathrm{AD}$ blends or clipping compounds, can be explained by the fact that the source words are fully preserved in WW blends and are not fully preserved in other formations used as stimuli in this experiment. The differences in recognition of the source words of AD blends and clipping compounds should be explained by other factors, as both AD and AC formations contain only parts of their source words, i.e. splinters. First of all, SW2 of $\mathrm{AD}$ blends have to be recognised not by their beginning, but by their final letters, unlike SW2 of clipping compounds, and word onsets are generally recognised more easily, as was discussed in Chapter 5. However, the absence of SW2 onset in AD blends is compensated by having more of SW2 present in the blend, and also by the overall prosodic shape which AD blends tend to retain from SW2, as suggested by the findings e.g. in Piñeros (2004) and Gries $(2006,2012)$. Indeed, all the AD blends which were selected as stimuli for this experiment, have the same number of syllables and the same main stress position as their SW2, except renoviction $\leftarrow$ renovation + eviction, which retains the prosodic contour of SW1. The degree of preservation of SW2 in AD blends may not be enough to ensure the same degree of recognisability as in WW blends, but appears to be higher than in AC formations. Moreover, the results of Task 1 show that SW1 of AD blends is correctly identified more often than SW1 of clipping compounds.

An important difference between my experiments and those described in Lehrer (2003, 2007) and Lehrer and Veres (2010) is that the stimuli selected for the present study included only novel blends and clipping compounds. Therefore, identifying the source words of blends and clipping compounds, the participants were likely to rely on the orthographic form of primes, rather than on context, or on prior knowledge. It has to be noted that I did not explicitly ask the participants whether or not they had been familiar with the words before seeing them in Task 1. This was not done for two reasons. First, such a debriefing session would make the experiment overly long. Second, the stimuli selected for the experiment are so novel that the likelihood of them being known to more than a handful of participants was very low.

In this study, it was assumed that the results of the identification and production task would have an influence on the priming effect revealed in Task 2. It was hypothesised 
that as a result of recognising the source words from blends in Task 1, the recognition of the same source words in Task 2 would be facilitated. The results of Task 2 confirm the priming effect of WW and AD blends on the recognition of their source words. It is also confirmed that the priming effect is stronger for WW blends than for $\mathrm{AD}$, as was predicted. As for clipping compounds, no priming effect on the recognition of their source words was predicted. The experiment results did not only show that, in accordance with hypothesis $2, \mathrm{AC}$ formations do not prime their source words, but they also demonstrated that, in fact, there is an inhibitory effect of exposure to AC formations. Such an effect is in line with the finding that the source words of AC formations were most difficult to identify in Task 1. It was discussed in Chapter 5 that the source words of $\mathrm{AC}$ are difficult to recognise because the splinters in AC formations are cut off too early to reach the recognition point (as shown in Gries (2006) on the basis of corpus data). The results of the identification and production task show that the source words of at least some novel clipping compounds can be correctly identified (in Group 2, more than in half cases). This may be due to the fact that AC primes contain splinters which appear also in other blends or clippings (as noted in section 7.3). Nevertheless, it appears that the source words of AC primes are less easily identified than those of AD blends. If so, it is possible that several words were considered as candidates to be named in Task 1, and therefore, more than one word was activated (in accordance with the cohort model of word recognition, see Chapter 5 and references therein). Competition between those words could be a reason why the source words of AC primes were recognised more slowly. It is also important to note that in the regression analysis of the reaction time in Task 2, the priming condition (i.e. whether a prime was shown in Task 1 or not) turned out to be a significant predictor of the response latency, but no significant effect of the actual response in Task 1 was found. It appears that the exposure to primes in Task 1 may result in facilitating the response to the corresponding source words in Task 2 even if these source words were not named correctly. It is possible, therefore, that the source words of higher transparency blends for which the priming effect was observed (i.e. WW and AD) were activated in Task 1 , even if the participants did not actually name them.

It may be argued that the priming effect observed in the experiment was due to orthographic similarity between blends and their source words. However, prime type, rather than similarity, turned out to be a significant predictor of both the reaction time to word targets, and the proportion of misrecognised words. The error analysis of nonword data demonstrates the difference between nonwords and words in this 172 
respect, and gives further evidence that the priming effect of blends on the recognition of their source words is due to the activation of the representations of words during Task 1, rather than to mere orthographic similarity between blends and their source words.

Exposure to AD and WW primes in the identification and production task was found to facilitate the recognition of word targets, but was shown to inhibit the recognition of nonwords orthographically similar to primes. This may be the consequence of high resemblance of WW-like nonword targets to real words. As discussed earlier, word and nonword targets were matched in orthographic similarity to primes. For example, both the word target rumour (SW1 of an AC prime rumint) and the corresponding nonword *rumacks begin with the same letter string (rum-) as the prime. Likewise, the nonwords * picknell and *rackade contain the same amount of orthographic material of the AD prime pickade, as its source words picket and blockade. The nonwords matched with the source words of WW targets, therefore, look very similar to words (compare aggravation and *garravation, text and *twext), and for this reason it may take more time to distinguish them from real words. The inhibitory effect of AC primes on nonword targets may be due to the fact that they share the initial letter string with the corresponding source words, which means that activating one or more real words from the initial cohort could cause a delay in recognition. The inhibitory effect of AD primes was smaller than that of WW and AC primes, which may be due to the fact that only half of $\mathrm{AD}$ primes share initial letter strings with nonwords. The other half of AD primes which share the final letter string with nonword targets do not affect the recognition of their source words in the same way as AC primes. This is, however, an assumption only, because the difference between targets similar to SW1 and SW2 of primes did not appear significant in any of the analyses, perhaps due to the limited number of primes.

In sum, the experiment presents evidence for the differences between blends and clipping compounds, not only in terms of their form, but also in terms of the way they are processed. The results of the identification and production task show that the degree of formal transparency of different types of blends determines whether or not their source words can be easily identified. The results of the lexical decision task demonstrate that the degree of formal transparency of primes influences the effect the primes have on the speed of the recognition of their source words. The results of the experiment imply that priming effect of blends on the recognition of their source words 
is related to the activation of the source words during the processing of blends, rather than to orthographic similarity between the source words and the blends. 


\section{Chapter 8. Synthesis and conclusions}

The aim of this thesis was to study blends as a phenomenon of contemporary word formation in English. In particular, the thesis aimed to explore the formal regularities of blends, and their relation to other word formation categories. A definition of blends was provided in Chapter 3, based on a systematic analysis of the relevant literature. This definition outlines the criteria for distinguishing blends from compounds and derivatives, but does not specify the criteria for distinguishing them from clipping compounds. As is clear from the analysis of linguistic studies of blends presented in Chapter 2, such a distinction requires us to consider various aspects of blend formation: their formal features alongside their semantics, and issues to do with how they are processed. The primary aim of the present research was to provide a description of blends which reflects the cognitive mechanisms involved in their formation, and which is also relevant for defining their place in contemporary English word formation.

\subsection{The design of this research revisited}

Blends are formally diverse to such an extent that generalisations about their formation made on purely formal grounds are often controversial, or even lead to the conclusion that no sensible generalisations about blends are possible at all. The argument about whether blends are a marginal morphological phenomenon or a productive type of word formation regulated by predictable constraints is still continuing, although many researchers now agree that blends are less marginal than used to be believed. A number of studies (Kubozono, 1990; Kelly, 1998; Gries, 2004a, 2006; Arndt-Lappe and Plag, 2013) have presented extensive evidence that the process of blend formation involves a considerable degree of regularity and predictability. This predictability concerns, in the first place, the phonological and phonotactic structure of blends. In particular, it has been shown that the formation of blends follows the same general rules that any English word, monomorphemic or polymorphemic, is bound to follow (compare fin-lit (AC) and shress (AD) with unlit and shrink). Thus, phonotactically, blends are not marginal. A number of publications on blends discussed in Chapter 2 also provide criteria of wellformedness that are applicable to blends and that can be used to describe the formation of prototypical blends. Among the criteria of well-formedness that are named in various studies are the degree of preservation of the source words (e.g. Algeo, 1977; Cannon, 1986; Kubozono, 1990; López Rúa, 2004), the degree of overlap (e.g. Adams, 1973; Algeo, 1977; Kelly, 1998; Brdar-Szabó and Brdar, 2008), and the preservation of the prosodic contour of the source words (Gries, 2012; Arndt-Lappe and Plag, 2013). All 
these criteria can be related to the degree of recognisability of the source words of the blends. This is important for the distinction between blends and clipping compounds, which was one of the areas of interest in the present research. Finding reliable criteria for this distinction has been problematic for many studies which either focussed specifically on blends, or only touched upon them in broader contexts. As a result, a number of studies (e.g. Bryant, 1974; Cannon, 1986; Borgwaldt et al., 2012) exclude clipping compounds from their scope on purely formal grounds, that is, because clipping compounds include the initial part of the second source word, unlike more frequently encountered blends which include the final part or the whole of the second source word.

The review of the literature presented in Chapter 2 revealed some reasons why such a formal distinction is arbitrary, and why it is important to take into account more than simply formal considerations when discussing the differences between blends and clipping compounds. Some of the studies which are not purely taxonomic in nature address possible reasons that may underlie the differences between blends and closely related word formation categories (such as clipping compounds). In particular, it is essential to consider the formal regularities of blends alongside the cognitive mechanisms involved in their formation, as suggested in Gries $(2006,2012)$. The aim of bringing the description of blends in line with what is really observed in contemporary language can justify considering blends and clipping compounds as prototypes rather than as clearly delimited categories. A prototypical approach is taken, for example, in López Rúa (2004), and is also earlier discussed in Bauer (1998) in relation to compounds. The approach taken in the present research (as also described in detail in Chapter 3) was to find grounds for placing blends on a continuum of word formation categories, based on psycholinguistic and cognitive considerations. To achieve this, the research combined corpus data analysis with the experimental study of the recognition of the source words of blends and clipping compounds. What makes this study different from earlier experiments on blends is 1) the principles of selecting targets, that is, focussing on novel formations, and 2) comparing blends to clipping compounds.

\subsection{The main findings of the thesis}

The analysis of the phonological, structural and semantic properties of contemporary blends (Chapter 4) provided a description and classification of blends, and criteria distinguishing them from compounds, derivatives, and clipping compounds. A collection of neologisms created by merging together two or more (up to four) words was 
described and classified into structural types. The principles underlying the formal classification into structural types are based on the approach developed in Plag (2003), and also taxonomic studies on blends such as Algeo (1977), Cannon (1986) and Bertinetto (2001). What distinguishes the classification suggested in this thesis is that, in addition to a practical description of the morphological phenomenon under consideration, the classification aimed to reflect psycholinguistically relevant features of blends, primarily the recognisability of their source words. As stated in Gries (2006), clipping compounds differ from blends in terms of the recognisability of their source words because the splinters included in clipping compounds are, on average, too short to allow for successful recognition of the source words. The present research builds on these findings, also taking into consideration the findings of psycholinguistic studies on word recognition (e.g. Johnson-Laird, 1987; Luzzatti et al., 2001; Whitney, 2001; Mondini et al., 2002; Badecker, 2007; Scaltritti and Balota, 2013). In particular, the parameters which are considered crucial for recognisability of the source words are 1) preserving the initial vs. the final parts of the source words, and 2) full vs. partial preservation of the source words. The formal classification worked out in Chapter 4 was based on the first of these parameters, but the categorical distinction between full or partial preservation was not made. That is to say, the AD structural type was assumed to include not only blends preserving the beginning of W1 and the end of W2, e.g. negatude $\leftarrow$ negative + attitude, but also blends preserving the whole of $\mathrm{W} 1$ and the end of $\mathrm{W} 2$, e.g. cheapuccino $\leftarrow$ cheap + cappuccino. Similarly, the AC structural type was assumed to include not only formations preserving the beginning of $\mathrm{W} 1$ and the beginning of $\mathrm{W} 2$, e.g. rumint $\leftarrow$ rumour + intelligence, but also formations preserving the whole of $\mathrm{W} 1$ and the beginning of W2, e.g. blogfic $\leftarrow$ blog + fiction. This was justified for the corpus study reported in Chapter 4, because 1) the length of the splinter and the proportion of each of the source words retained in a particular blend were also taken into account, and 2) it was important to impose as few arbitrary decisions as possible on the classification into structural types. In other words, the category of structural type was used not for the sake of classification per se, but, rather, to help describe the mechanisms that underlie blend formation.

The analysis presented in Chapter 4 showed that blend words are different from clipping compounds in various aspects, apart from purely formal ones. Mainly, the two types of word formation differ in terms of the position of the switch point in the syllabic structure, and also in terms of the relative frequency of co-occurrence of the source 
words. The fact that a relatively high frequency of co-occurrence of two words may result in these words forming a clipping compound can be explained in terms of exemplar theory (Pierrehumbert, 2001; Bybee, 2006; Goldberg, 2006). According to exemplar theory, the representations of particular words, collocations, phonetic or sematic features, etc., which are stored in memory influence subsequent language use, and the strength of their influence depends on how frequent and how recent the representations are. For example, the more frequently collocating words are used together, the more likely it is that this collocation undergoes phonetic reduction. What is observed with clipping compounds is more complex than the phonetic reduction described in Bybee and Scheibman (1999) and Bybee (2001). In clipping compounds, the source words are reduced to their shorter versions to a greater extent than would be the case if it were only a result of fast articulation.

The results of the corpus study in Chapter 4 implied that the process of merging two words into one is driven by two factors: relatively frequent co-occurrence of these two words, or the possibility to merge them in one in such a way that both remain recognisable. It was found that blends, unlike clipping compounds, are more likely to be formed not as a result of simple co-occurrence of their source words, but as a result of some other process, such as a conscious attempt to bring them together to express a specific meaning. The analysis in Chapter 4 provides evidence in support of findings reported by, for example, Kubozono (1990) and Gries (2006, 2012). This contribution to earlier findings is especially important because the conclusions in Chapter 4 were arrived at using different principles of data collection and different methods to those in the studies above. The conclusions concerning different degrees of recognisability of blends of different structural types based on the results of corpus analysis had to be further verified in an experimental study.

The principles and methods of such an experimental study were discussed in Chapter 5. A review of literature on word recognition was presented, and the experimental design appropriate for the aims of the present research was elaborated. Two experiments were carried out in the course of the present research, each designed in accordance with the aims of the research outlined above. The structural classification developed in Chapter 4 was used for selecting the stimuli for both experiments. In order to account for possible effects of full / partial preservation of the source words, this structural distinction was introduced for the experimental stimuli. 
The experiment presented in Chapter 6 was a web-based survey during which native speakers of English were asked to read sentences containing blends and clipping compounds, and to evaluate the definitions of their source words. The evaluations of four types of definitions were compared: 1 ) those containing both source words; 2) those containing only W1;3) those containing only W2;4) those containing neither of the source words. The study results indicate that the structural type of the target words (i.e. whether they are blends retaining both their source words in full, blends retaining only one of the source words in full, or none at all, or clipping compounds) influences the readers' evaluation of the definitions of these words. In particular, the definitions of blends which preserve at least one of the source words in full (e.g. clapathy $\leftarrow$ clap + apathy, bigature $\leftarrow$ big $+($ mini)ature, approximeeting $\leftarrow$ approxim(ate) + meeting), were given significantly higher evaluations than the definitions of clipping compounds (rumint $\leftarrow$ rumour + intelligence), or AD blends that preserve neither of their source words in full (e.g. pickade $\leftarrow$ picket + blockade). This tendency was observed regardless of the definition type. Moreover, the survey results have shown that the presence of the source words in the definition influenced the evaluations in a different way, depending on the structural type of the defined word. The definitions of clipping compounds and $\mathrm{AD}$ blends were given a higher rating if the source words were given in the text of the definitions. This effect was not observed for WW blends, which preserve both the source words in full. It appears that the readers did not need the presence of the source words in a definition of a WW blend to decide how successfully the definition explained the meaning of the blend. Such a result indicates that the source words are recognisable from the form of WW blends well enough not to be needed in the definition. This conclusion is also supported by the numeric differences in the evaluations of definitions of AW and WD blends. If a source word was not fully present in the blend (as, for example, miniature in the WD blend bigature, or approximate in the AW blend approximeeting), its presence in the definition resulted in a higher evaluation of that definition. As for clipping compounds, and also blends which preserve neither of their source words in full, the presence of both the source words in the definitions of these words appears to be important for the definitions to be given high evaluations. It has to be noted that the definitions of clipping compounds containing neither of their source words were given lower evaluations than the same type of definitions of AD blends. On the other hand, the definitions of clipping compounds containing both the source words were given higher evaluations than the same type of definitions of $\mathrm{AD}$ blends. These 
numeric differences between the evaluation scores for clipping compounds and AD blends suggest that the source words of clipping compounds are less recognisable than the source words of AD blends. The results of the survey implied 1) that full preservation of the source words in blends is an important factor influencing their recognition, and 2) that the differences in recognisability between AD blends and clipping compounds had to be further explored.

In order to reveal the difference in recognisability between AD blends and clipping compounds, and also to find further confirmation of the above results, a psycholinguistic experiment was carried out. The purpose of the experiment described in Chapter 7 was to study the effect which recognising the source words from the form of blends and clipping compounds had on the recognition of those words in a lexical decision task. The experimental design was inspired by earlier studies by Lehrer $(1996,2007)$. Unlike Lehrer, I did not aim to find evidence of rapid and automatic decomposition of blends into their source words. Instead, the evidence of a long-term priming effect of blends and clipping compounds on the recognition of their source words was sought. The conditions for long-term priming were created by combining an identification and production task and a lexical decision task in one experiment. Another way in which this study differs from Lehrer's, and also from the other experimental studies on blends, e.g. Tomaszewicz (2012), is that it compares blends to clipping compounds, which is not usually done. For such comparison to be made possible, experimental stimuli included clipping compounds and AD blends which did not fully preserve any of their source words. As it was also essential to control for full preservation of the source words, a group of WW blends fully preserving both the source words was also included in the set of experimental stimuli.

The first task of the experiment was an identification and production task, during which the participants were asked to identify the source words of blends and clipping compounds displayed on the screen. The analysis of the responses showed that the source words of fully overlapping blends (e.g. predictionary) were retrieved more easily than those of non-overlapping blends (e.g. renoviction), and that the source words of clipping compounds (e.g. finlit) were the most difficult to identify. In a subsequent task, the participants were shown the source words of blends and clipping compounds and were asked to decide whether or not they were real words in English. The main finding of this experiment is as follows. If the participants were shown a blend in the 
identification task, then it facilitated the recognition of its source words in the subsequent lexical decision task. Importantly, this priming effect was stronger for fully overlapping blends, weaker for non-overlapping blends, and no priming effect was found for clipping compounds. Moreover, it was observed that exposure to clipping compounds in the identification task resulted in slower recognition of their source words in the lexical decision task (compared to blends).

An analysis of the responses to nonword targets matched with the word targets in terms of orthographic similarity to the primes (i.e. the blends and clipping compounds presented in the identification task) provided an explanation of the observed differences in priming effects for blends and clipping compounds. First, it has to be pointed out that the exposure to primes in the identification and production task resulted in slower responses to the matched nonword targets. This effect was observed for all three types of primes, but it was strongest for nonword targets orthographically similar to clipping compounds. The responses to nonword targets orthographically similar to AD blends and WW blends were generally slower than the responses to nonwords similar to clipping compounds, whether the primes were shown or not, and prior exposure to clipping compounds resulted in slower responses to the matched nonwords. Such results can be explained by the effect of orthographic similarity between nonword targets and real words. As shown, for example, by Grainger and Jacobs (1996), if nonword targets have orthographically similar neighbours among real words, that is, if they are more word-like, they take longer to reject as not being words. Because the nonword targets in the present experiment were matched with the primes for orthographic similarity, the nonword targets matched with WW blends were, as a result, the most word-like, because the degree of preservation of the source words in WW blends was the highest. This can explain the slow reaction to nonwords matched with WW primes. The nonword targets matched with AD blends were, accordingly, less wordlike, and the nonword targets matched with clipping compounds, the least word-like of all the nonwords among the present experimental stimuli. This means that the amount of material from the beginning of a real word preserved in such nonword targets (e.g. fin-from financial, preserved in the nonword target *finerniel) does not make the nonwords word-like enough to cause the similar inhibitory effect to that observed for other prime types. This is also compatible with the results of the identification task showing that the source words of clipping compounds are harder to identify than the 
source words of blends, which suggests that the amount of the source words that is preserved in clipping compounds is not enough for their identification.

Given that nonword targets matched with clipping compounds were not similar enough to real words to be perceived as word-like, exposure to clipping compounds might have caused the participants to begin treating such nonwords as word-like, and therefore to be more reluctant to reject them as words. This can be explained by the fact that both clipping compounds and the matched nonwords have beginnings similar to other English words. Presenting clipping compounds in the identification and production task, and moreover, the nature of the task itself, i.e. asking the participants to identify the source words of the presented formation, might cause the activation of multiple words with similar beginnings in the participants' mental lexicon. In terms of the Cohort Model of word recognition (Marslen-Wilson and Welsh, 1978; Marslen-Wilson, 1987), presenting a clipping compound in an identification task might cause the activation of the word-initial cohorts of its source words. This, in turn, could be the reason for the slower reaction to the nonwords starting with the same letter string. It is important to note that the activation of the word-initial cohorts happens irrespective of the actual response (or lack thereof) in the identification task. Even if the relevant source word of a clipping compound was not named (or named incorrectly) in the identification task, the activation of one or more words beginning with the same letter string as the nonword target results in slower rejection of that nonword target. The same applies to the recognition of the source words of clipping compounds. The possibility of activation of more than one word from the relevant word-initial cohorts can explain the inhibitory effect of clipping compounds as primes on the recognition of their source words.

The results of the experiment have shown that presenting the prime words in an identification task had different effects on the recognition of their source words and on the processing of orthographically similar nonwords. Therefore, it can be concluded that the observed priming effect is due not purely to orthographical similarity between blends and their source words, but, rather, to activation of the representations of the source words during the processing of blends. The results can be used to re-interpret earlier findings of Lehrer (1996, 2007), and Lehrer and Veres (2010): it is confirmed that a direct retrieval task works better for facilitating the recognition of the source words than masked priming. 
In sum, the results of the present research reveal that blends differ from clipping compounds not only formally, but also in terms of their processing. In addition to the distinction between blends and clipping compounds, the differences between different types of blends were demonstrated. High degree of formal transparency (e.g. in WW blends like predictionary) results in higher recoverability of their source words. The source words of AD blends with a low degree of formal transparency (e.g. blizzaster) are less recoverable, and clipping compounds like globfrag are characterised by lower recoverability of the source words than even low transparency blends.

The findings of the research show that it is practical to define the criteria of wellformedness of blends in terms of the recognisability of their source words, which is determined by the degree of similarity between the source words and the resulting formations, the preservation of beginning or ending, the retention of the prosodic pattern, and full or partial preservation of the source words. In the light of these findings, a typical blend can be defined as a lexical item formed by merging together two source forms, so that 1 ) only part of their orthographical and/or phonological material is preserved, and 2) the full constituents remain retrievable from the blend. The second part of this definition is hard to use for practical purposes of classification. Therefore, the definition based on formal criteria which is provided in Chapter 3 is more suitable for taxonomic purposes. This definition, however, should be complemented by a formal criterion distinguishing blends from clipping compounds, as given below:

A blend is a lexical item formed by merging together two source forms, so that 1) it includes the beginning or the whole of the first word, and the ending or the whole of the second word, 2) only part of their orthographical and/or phonological material is preserved, 3) no transparent analysis into morphs is possible.

The results of this research suggest that including the formal criterion 1) in the definition above is not an arbitrary decision, but is based on the psycholinguistically relevant characteristics of blend words which are important factors of their wellformedness. The definition above does not cover cases of central replacement blends such as intextication $\leftarrow$ intoxication + text, or blends formed of more than two source words such as Christmahanukwanzadan $\leftarrow$ Christmas + Hanukkah + Kwanza + Ramadan. This is because, firstly, blends like these are less typical, and secondly, working out the criteria of recognisability of the source words in such blends was outside the scope of 
the present research. Nevertheless, even formations which do not conform to all the formal criteria given in the definition above can be referred to as blends if their source words are recognisable, in line with the general considerations of well-formedness outlined in this thesis.

\subsection{The implications of the research}

This research combined the analysis of corpus data with the study of the responses of participants in two experiments. As a result, the findings of the research cover a wide range of facts related to the phenomenon of blending. These findings have implications for both descriptive and taxonomic studies in morphology, as well as psycholinguistic studies of word processing.

\subsubsection{Finding room for blends in English morphology: Implications for taxonomic studies}

One of the aims of the present research was to distinguish between blends and the neighbouring word formation categories, primarily clipping compounds. The common feature of compounds, fully overlapping blends, partially overlapping blends and clipping compounds is that in all of these formations two or sometimes more words are joined in a single lexeme. The difference between these formations is in the degree of shortening of the original constituents: from no shortening at all in compounds, to substantial shortening in clipping compounds (an extreme degree of shortening would in this case be represented by acronyms). This conceptualisation of the word formation categories is very similar to the 'categorical continuum' described in López Rúa (2004b), and also to the conclusions regarding compounds in Bauer (1998), arrived at using different data and different methodology. The present research has also revealed that the degree of shortening (and therefore, the recoverability of the source words from the resulting formation) is related to the frequency of co-occurrence of the source words. Such characteristics as the degree of shortening and the frequency of co-occurrence are continuous values, rather than categorical distinctions. Therefore, in light of the findings presented in the thesis, the distinction between blends and other types of formations which combine various parts of words into one lexeme seems not categorical, but, rather, involving points on a continuum.

This thesis presented strong evidence for the distinction between blends and clipping compounds. This distinction, however, is of a different nature to that made, for example, 
in Gries (2006, p. 536) wherein blends, clipping compounds and acronyms are classified as 'other' types of word formation, different both from derivation and compounding. What is important about the present findings is that, in addition to the distinction between blends and clipping compounds, significant differences were revealed between blends containing full source words and blends containing only shortened versions of the source words. In sum, the research presented evidence in support of a prototypical approach to relations between different types of blends. One of the questions raised in this thesis is whether the primary mechanism of blending is addition (as in compounding) or shortening. The results of this research have shown that with respect to blends this should not be a categorical distinction. The formation of blends that fully preserve both of their source words can be analysed as compounding rather than shortening. On the other hand, the formation of clipping compounds can be analysed as a type of shortening. These results imply that blends (at least in English) should be analysed as a productive type of word formation, as suggested, for example, in Plag (2003), rather than an extragrammatical and unpredictable way of creating new words, as claimed in Dressler (2000) and Mattiello (2013).

The implications of the present research go beyond a simple distinction between blends and clipping compounds. The dissertation outlines a number of distinctive features of various types of blends which, as a result of the study, can be pictured as points in a continuum space of formations governed by two processes: compounding and clipping. This continuum can potentially embrace those data points which for their marginality have been excluded from the analysis (e.g. SoLoMo $\leftarrow S o($ cial $)+L o(c a l)+M o(b i l e)$ and other three-constituent formations, and various acronym-like formations). In this light, one of the key implications of this research is in revealing psycholinguistically relevant differences between items with different degrees of shortening of the original constituents: from no shortening at all in compounds, to a substantial shortening in clipping compounds (an extreme degree of shortening would in this case be represented by acronyms). This conceptualisation of the word formation categories is similar to what is postulated in Bauer (1998), and in López Rúa (2004), and the results of the present research comprise empirical evidence supporting the claims therein. 


\subsubsection{Finding room for blends in your 'mind palace': Implications for the studies of word processing}

One of the key findings of the present research is that formal transparency (related to the recognisability of the constituents) not only distinguishes between blends and clipping compounds, but is also essential for the processing of these formations. Moreover, the results of this research suggest that the prototypicality of blends is determined by the degree of recognisability of their source words. The structural type of blends, as defined in this thesis, directly reflects two features that determine the degree of recognisability of the source words from the form of the blend: full or partial preservation of the source words, and preserving the initial or final part of the source words. Other factors which were found to be related to recognisability are to a large extent reflected in the structural type of blends. One such factor is the proportion of the source words preserved in the blend, which, by definition is the highest in WW blends, and tends to be lower in clipping compounds than in blends of any type (as shown in Chapter 4). The findings of two experimental studies reported in the thesis concerning the recognition of the source words in blends and clipping compounds have implications not only for further research on blends and clippings. More importantly, the findings of this thesis can be used for the development of models of word processing (such as the Cohort Model (Marslen-Wilson, 1987) of word recognition).

The recognisability of blend constituents can also be related to the degree of similarity between the blend and its source words, partly reflected in the phonological and / or graphical overlap which characterises some of the blends (primarily, the WW type). In the studies of blends, similarity is discussed in terms of the degree of overlap (Algeo, 1977; Kelly, 1998; López Rúa, 2004b), and also using more complex measures of similarity such as the degree of phonetic similarity of the neighbouring sounds (Kelly, 1998), and the number of graphemic and phonemic n-grams shared by blends with their source words (Gries, 2012). In Gries (2006), recognisability is discussed in terms of the switch-point distance, which is the distance between the 'uniqueness point' after which the source word becomes easily recognisable among all the other words beginning or ending with a given letter string and the actual switch point in a blend. The switch point distance can be argued to also reflect the degree of similarity between the source words and the blend. A number of similarity measures, including the switch point distance, were used in the present study to evaluate the influence of similarity on the response in 
the experimental tasks. In the course of data analysis it was found that these similarity measures correlate highly with each other, and that different similarity measures turned out to be optimal for different types of analysis. This indicates that, effectively, any of the similarity variables discussed in Chapters 6 and 7 can be used to estimate similarity between two given words. Moreover, the analysis has shown that a single measure is often not enough, as such factors as the length of the shared phonological and graphical segments, the presence or absence of the shared beginning, and also the overall syllable length and the stress position can be relevant for different formations, depending on their structure.

Another feature which is associated with recognisability is the prosodic pattern of the source words, i.e. the number of syllables and the position of the main stress. The role of prosodic structure as a factor that enhances recognisability is discussed, for example, in Gries (2004a, 2006), Renner (2006), Bat-El and Cohen (2012), Arndt-Lappe and Plag (2013) primarily on the basis of collections of blends defined here as AD. In the current study the prosodic similarity between various types of blends and their source words is considered, and blends are compared to clipping compounds in this respect.

Another factor which is important for the recognition of words (and also other items, both at the sublexical and supralexical level) is frequency. Frequency effects can be discussed in relation to productivity (Bauer, 2001; Plag, 2006), and also in relation to informativity (see, for example, Bell and Plag (2012) for a discussion of frequency as an informativity effect in compounds). In this research, the frequency of individual phonemes was found to be related to the length of splinters in blends, which suggests that some phonemes are more informative than others. Word frequency and splinter frequency turned out to influence the recognition of the source words in the lexical decision task, as demonstrated in Chapter 7. This is not simply a further confirmation of word frequency effects discussed in many publications on word recognition. What is more important, the relative splinter frequency, as defined in Chapter 6, can be a useful factor to be considered in studies on the productivity of splinters, combining forms, and affixes. The role of the frequency of word constituents that is discussed in the thesis is often considered in contemporary studies which apply a probabilistic approach to linguistic phenomena (e.g. Feldman et al., 2004; Hay and Baayen, 2005). The results of this research provide further evidence that probability plays an important role in word formation and justify the inclusion of probability effects in the discussion of 
morphological phenomena, e.g. in the framework of exemplar theory (Goldberg, 1995; Pierrehumbert, 2001; Bybee, 2006).

\subsection{The limitations of the study and the recommendations for future research}

As pointed out above, this research combined a variety of methods of data collection and analysis. The thesis reported the results of three separate studies looking at different aspects of one phenomenon. While this is a clear strength of the approach taken in the thesis, it is also inevitable that the combination of such different tasks in one piece of research means that I was less well able to engage deeply in any of them. For example, in terms of formal analysis, to provide a complete and exhaustive account of all the phonetic and phonological factors involved in blend formation would probably require a whole PhD-level study, and the same applies to all aspects of the processing of blends. The task of getting a view of the phenomenon from different perspectives implied that the depth of the analysis was limited to some extent. One of the limitations in this respect, as was pointed out in section 4.2 , is that the association between the frequency /sonority ranking of the phonemes was considered only for the two boundary phonemes, rather than for all phonemes in the splinter. Because of this, it is not possible to determine within the context of the present research whether the observed correlations are representative of the tendencies working along the whole splinter, or whether they are only valid for the phonemes positioned close to the switch point.

Perhaps one of the most important limitations of this study is that semantic aspects of blending did not provide a main focus of the thesis. Both syntagmatic origin blends (that is, blends which have semantic heads) and paradigmatic origin blends (also termed coordinative blends) were included in the data for this study. However, a more subtle semantic classification, or a discussion concerning which semantic relations are more prototypical in blends did not form part of my approach. Some of the findings in this thesis suggest that the semantic factors and the formal structure of blends are related. For example, it was found that WW blends are almost categorically headed, and the source words of AD blends tend to be in coordinative relations. This can be regarded as a problem for the discussion of the well-formedness of blends. If the coordinative relations between the source words are viewed as one of the criteria of wellformedness, as in Kubozono (1990), Kelly (1998) or Renner (2006), then WW blends are less typical than $\mathrm{AD}$ blends. A more detailed analysis of the interaction between the structure and the semantics of blends should be considered in further research. 
The recognisability of the source words in blends was discussed primarily in terms of orthographic and phonological factors; what was not considered in this thesis is the degree of morphological relatedness between blends and their source words. For example, in the analysis of responses to Task 1 of the experiment described in Chapter 7 , morphologically related responses such as globular instead of globalisation were treated as incorrect. However, it could be argued that morphologically related responses should be treated differently from unrelated responses. The analysis presented in Chapter 7 shows the priming effect of exposure to blends, whether or not the responses were correct or not, but it could be the case that morphological relatedness also influences the recognition of blend constituents (see, for example, Marslen-Wilson et al. (1994), Schreuder and Baayen (1995), Rastle et al. (2000) for discussion of morphological effects in word processing). It has to be noted that the experimental stimuli used in the present study are not the best material for the study of morphological relatedness. The relevant data that can be extracted from my experiment (that is, the words named in Task 1 that do not share lemmas with the source words, but are morphologically related) are too scarce to reveal any patterns if they exist at all. It would take another experiment, with a different design and different stimuli, to look into the effects of morphological relatedness in blends.

It was pointed out more than once throughout this thesis that the productivity of a splinter influences the recognition of the words formed with that splinter, and the higher the productivity, the weaker the semantic link between the splinter and the word it originates from, e.g. -holic and alcoholic. An increase in productivity can result in the grammaticalisation of the splinter and turning it into a combining form or an affix, so that new formations with such splinters will be formed by derivation, not blending. A thorough study of the productivity of splinters was beyond the scope of the present investigation, but it turned out that considering the productivity of splinters is one of the necessary prerequisites of a study of blends. Selecting data for the structural and phonological analysis in Chapter 4, and also stimuli for the experiment in Chapter 7, I aimed to filter out formations with productive splinters, but even the steps that were taken could not guarantee that the effect of productivity was entirely controlled for. An alternative approach would be to carry out an experimental study with made-up blends and clipping compounds, created with regard to the factors which have been found to influence the formation of real blends and clipping compounds (including the factors discussed in the present thesis). 
To conclude, this research, as is frequently the case, raises a number of questions to be answered in future studies, alongside answers to the questions which inspired it. The thesis contains a number of important findings concerning the formation, structure and processing of blends, but it by no means exhausts the subject of blending in English. Finally, it provides some openings for future investigations in morphology and psycholinguistics. 


\section{References}

Adams, V. (1973). An introduction to modern English word formation. London: Longman.

Adams, V. (2001). Complex words in English. Harlow: Longman.

Aitchison, J. (2002). Words in the mind: An introduction to the mental lexicon (3rd ed.). Oxford: Blackwell Pub.

Algeo, J. (1977). Blends, a structural and systemic view. American Speech, 52, 47-64.

Algeo, J. (Ed.). (1993). Fifty years among the new words: A dictionary of neologisms 19411991 (2nd ed.). New York \& Oakleigh: Cambridge University Press.

Arndt-Lappe, S., \& Plag, I. (2013). The role of prosodic structure in the formation of English blends. English Language and Linguistics, 17(3), 357-563. doi:10.1017/S1360674313000154

Baayen, H. R. (2008). Analyzing linguistic data. Cambridge University Press.

Baayen, H. R., \& Milin, P. (2010). Analyzing reaction times. Journal of Psychological Research, 3(2), 12-28.

Baayen, H. R., \& Neijt, A. (1997). Productivity in context: A case study of a Dutch suffix. Linguistics, 35(3), 565-587.

Baayen, H. R., Schreuder, R., De Jong, N., \& Krott, A. (2002). Dutch inflection: The rules that prove the exception. In S. Nooteboom, F. Weerman, \& F. Wijnen (Eds.), Storage and computation in the language faculty (pp. 61-92). Dordrecht: Kluwer.

Badecker, W. (2001). Lexical composition and the production of compounds: Evidence from errors in naming. Language and Cognitive Processes, 16(4), 337-366.

Badecker, W. (2007). Processing compound words: An introduction to the issues. Brain and Language, 103(1), 16-17.

Bat-El, O. (2000). The grammaticality of extragrammatical morphology. In U. Doleschal \& A. M. Thornton (Eds.), Extragrammatical and marginal morphology (pp. 61-84). München: Lincom Europa.

Bat-El, O. (2006). Blend. In K. Brown (Ed.), Encyclopedia of language and linguistics (pp. 66-70). Amsterdam: Elsevier.

Bat-El, O., \& Cohen, E.-G. (2012). Stress in English blends: A constraint-based analysis. In V. Renner, F. Maniez, \& P. J. L. Arnaud (Eds.), Cross-disciplinary perspectives on lexical blending (pp. 193-211). Berlin: De Gruyter Mouton.

Bauer, L. (1983). English word-formation. Cambridge: Cambridge University Press.

Bauer, L. (1998). Is there a class of neoclassical compounds, and if so is it productive? Linguistics, 36(3), 403-422. doi:10.1515/ling.1998.36.3.403

Bauer, L. (2001). Morphological productivity. Cambridge: Cambridge University Press.

Bauer, L. (2006). Compounds and minor word-formation types. In B. Aarts \& A. McMahon (Eds.), The handbook of English linguistics (pp. 483-506). Oxford: Blackwell. 
Bauer, L. (2009). Typology of compounds. In R. Lieber \& P. Štekauer (Eds.), The Oxford handbook of compounding (pp. 343-356). Oxford: Oxford University Press.

Bauer, L. (2012). Blends: Core and periphery. In V. Renner, F. Maniez, \& P. J. L. Arnaud (Eds.), Cross-disciplinary perspectives on lexical blending (pp. 11-22). Berlin: De Gruyter Mouton.

Bauer, L., Lieber, R., \& Plag, I. (2013). The Oxford reference guide to English morphology. Oxford: Oxford University Press.

Bauer, L., \& Renouf, A. (2000). Contextual clues to word-meaning. International Journal of Corpus Linguistics, 5(2), 231-258.

Beliaeva, N. (2014). A study of English blends: From structure to meaning and back again. Word Structure, 7(1), 29-54.

Bell, M. J., \& Plag, I. (2012). Informativeness is a determinant of compound stress in English. Journal of Linguistics, 48(3), 485-520.

Benczes, R. (2006). Creative compounding in English: The semantics of metaphorical and metonymical noun-noun combinations. Amsterdam \& Philadelphia: Benjamins.

Berg, T. (1998). Linguistic structure and change: An explanation from language processing. Oxford: Oxford University Press.

Bergström, G. A. (1906). On blendings of synonymous or cognate expressions in English: A contribution to the study of contamination. Lund: H. Osslon. Retrieved from http://www.archive.org/details/onblendingsofsyn00bergrich

Bertinetto, P. M. (2001). Blends and syllabic structure: A four-fold comparison. In M. Lorente, N. Alturo, E. Boix, M.-R. Lloret, \& L. Payrató (Eds.), La gramàtica i la semàntica per a l'estudi de la variació (pp. 59-112). Barcelona: Promociones y Publicaciones Universitarias.

Bertram, R., Laine, M., Harald Baayen, R., Schreuder, R., \& Hyönä, J. (2000). Affixal Homonymy triggers full-form storage, even with inflected words, even in a morphologically rich language. Cognition, 74(2), B13-B25. doi:10.1016/S00100277(99)00068-2

Blevins, J. P. (2003). Stems and paradigms. Language, 79, 737-767.

Bock, H.-H., \& Diday, E. (Eds.). (2000). Analysis of symbolic data: Exploratory methods for extracting statistical information from complex data. Berlin: Springer-Verlag.

Bod, R., Hay, J., \& Jannedy, S. (Eds.). (2003). Probabilistic linguistics. Cambridge, MA: MIT Press.

Böhmerová, A. (2010). Blending as lexical amalgamation and its onomatological and lexicographical status in English and in Slovak. Bratislava: ŠEVT.

Borgwaldt, S., \& Benczes, R. (2011). Word-formation patterns in a cross-linguistic perspective: Testing predictions for novel object naming in Hungarian and German. In D. Schönefeld (Ed.), Converging evidence: Methodological and theoretical issues for linguistic research (pp. 221-246). Amsterdam \& Philadelphia: Benjamins. 
Borgwaldt, S. R., Kulish, T., \& Bose, A. (2012). Ukrainian blends: Elicitation paradigm and structural analysis. In V. Renner, F. Maniez, \& P. J. L. Arnaud (Eds.), Crossdisciplinary perspectives on lexical blending (pp. 75-92). Berlin: De Gruyter Mouton.

Brdar-Szabó, R., \& Brdar, M. (2008). On the marginality of lexical blending. Jezikoslovlje, 9(1-2), 171-194.

Bryant, M. M. (1974). Blends are increasing. American Speech, 49, 163-184.

Bybee, J. (1995). Regular morphology and the lexicon. Language and Cognitive Processes, $10,425-455$.

Bybee, J. L. (2001). Phonology and language use. Cambridge: Cambridge University Press.

Bybee, J. L. (2006). From usage to grammar: The mind's response to repetition. Language, $82(4), 711-733$.

Bybee, J. L., \& Scheibman, J. (1999). The effect of usage on degrees of constituency: The reduction of don't in English. Linguistics, 37, 575-596.

Cannon, G. (1986). Blends in English word formation. Linguistics, 24(4), 725-753.

Cannon, G. (1987). Historical change and English word-formation. New York, Berlin, Frankfurt am Main \& Paris: Peter Lang.

Cannon, G. (1989). Abbreviations and acronyms in English word-formation. American Speech, 64(2), 99-127.

Chialant, D., \& Caramazza, A. (1995). Where is morphology and how it is processed? The case of written word recognition. In L. B. Feldman (Ed.), Morphological aspects of language processing (pp. 55-76). Hillsdale, NJ \& Hove, UK: Laurence Erlbaum Associates.

Cook, P., \& Stevenson, S. (2007). Automagically inferring the source words of lexical blends. In Proceedings of the 10th Conference of the Pacific Association for Computational Linguistics (PACLING 2007) (pp. 289-297). Melbourne, Australia.

Davies, M. (2008). The Corpus of Contemporary American English (COCA): 400+ million words, 1990-present. Retrieved from http://www.americancorpus.org/

Devereux, R. (1984). Shortenings, blends and acronyms. Word Ways, 17, 210-215.

Dormann, C. F., Elith, J., Bacher, S., Buchmann, C., Carl, G., Carré, G., Marquéz, J. R. G., Gruber, B., Lafourcade, B., Leitão, P. J., Münkemüller, T., McClean, C., Osborne, P. E., Reineking, B., Schröder, B., Skidmore, A. K., Zurell, D., Lautenbach, S. (2013). Collinearity: A review of methods to deal with it and a simulation study evaluating their performance. Ecography, 36(1), 27-46. doi:10.1111/j.1600-0587.2012.07348.x

Downing, P. A. (1977). On the creation and use of English compound nouns. Language, 53, 810-842.

Dressler, W. U. (2000). Extragrammatical vs. marginal morphology. In U. Doleschal \& A. M. Thornton (Eds.), Extragrammatical and marginal morphology (pp. 1-10). München: Lincom Europa. 
Fandrych, I. (2004). Non-morphematic word-formation processes: A multi-level approach to acronyms, blends, clippings and onomatopoeia ( $\mathrm{PhD}$ thesis). University of the Free State, Bloemfontein.

Fandrych, I. (2008a). Pagad, chillax and jozi: A multi-level approach to acronyms, blends, and clippings. Nawa Journal of Language and Communication, 2(2), 71-88.

Fandrych, I. (2008b). Submorphemic elements in the formation of acronyms, blends and clippings. Lexis: Lexical Submophemics / La Submorphémique Lexicale, 2, 105-123.

Fauconnier, G., \& Turner, M. (1998). Blending as a central process of grammar. Retrieved from http://www.cogsci.ucsd.edu/ faucon/BEIJING/blending-grammar.pdf

Fauconnier, G., \& Turner, M. (2002). The way we think: Conceptual blending and the mind's hidden complexities. New York: Basic Books.

Feldman, L. B., Soltano, E. G., Pastizzo, M. J., \& Francis, S. E. (2004). What do graded effects of semantic transparency reveal about morphological processing? Brain and Language, 90, 17-30.

Forster, K. I., \& Davis, C. (1984). Repetition priming and frequency attenuation in lexical access. Journal of Experimental Psychology: Learning, Memory, and Cognition, 10(4), 680-698.

Fradin, B. (2000). Combining forms, blends and related phenomena. In U. Doleschal \& A. M. Thornton (Eds.), Extragrammatical and marginal morphology (pp. 11-59). München: Lincom Europa.

Fromkin, V. A. (Ed.). (1973a). Speech errors as linguistic evidence. The Hague: Mouton de Gruyter.

Fromkin, V. A. (1973b). The non-anomalous nature of anomalous utterances. In V. A. Fromkin (Ed.), Speech errors as linguistic evidence (pp. 215-242). The Hague: Mouton de Gruyter.

Garrett, M. F. (1975). The analysis of sentence production. In G. H. Bower (Ed.), The psychology of learning and motivation (Vol. 9). New York: Academic Press.

Giegerich, H. J. (1992). English phonology: An introduction. Cambridge: Cambridge University Press.

Goldberg, A. E. (1995). Constructions. Chicago: The University of Chicago.

Goldberg, A. E. (2006). Constructions at work: The nature of generalization in language. Oxford: Oxford University Press.

Grainger, J. (1990). Word frequency and neighborhood frequency effects in lexical decision and naming. Journal of Memory and Language, 29, 228-244.

Grainger, J., \& Jacobs, A. M. (1996). Orthographic processing in visual word recognition: A Multiple Read-Out Model. Psychological Review, 103(3), 518-565.

Gries, S. T. (2004b). Shouldn't it be breakfunch? A quantitative analysis of blend structure in English. Linguistics, 42(3), 639-667. 
Gries, S. T. (2004b). Some characteristics of English morphological blends. In M. A. Andronis, E. Debenport, A. Pycha, \& K. Yoshimura (Eds.), Papers from the 38th Regional Meeting of the Chicago Linguistics Society (Vol. 2: The panels, pp. 201216). Chicago: Chicago Linguistic Society.

Gries, S. T. (2006). Cognitive determinants of subtractive word-formation processes: A corpus-based perspective. Linguistics, 17(4), 535-558.

Gries, S. T. (2012). Quantitative corpus data on blend formation: Psycho- and cognitivelinguistic perspectives. In V. Renner, F. Maniez, \& P. J. L. Arnaud (Eds.), Crossdisciplinary perspectives on lexical blending (pp. 145-167). Berlin: De Gruyter Mouton.

Gries, S. T., Achard, M., \& Kemmer, S. (2004). Isn't that fantabulous? How similarity motivates intentional morphological blends in English. In Language, culture and mind (pp. 415-428). Stanford, CA: Center for the Study of Language and Information (CSLI).

Hall, T. A. (1999). The phonological word: A review. In T. A. Hall \& U. Kleinhenz (Eds.), Studies on the phonological word (pp. 1-22). Amsterdam: Benjamins.

Halle, M., \& Marantz, A. (1993). Distributed morphology and the pieces of inflection. In K. L. Hale, S. J. Keyser, \& S. Bromberher (Eds.), The view from building 20: Essays in linguistics in honor of Sylvain Bromberger (pp. 111-176). Cambridge, MA: MIT Press.

Hay, J. B., \& Baayen, H. R. (2005). Shifting paradigms: Gradient structure in morphology. Trends in Cognitive Sciences, 9(7), 342-348.

Hofmeister, P. (2011). Representational complexity and memory retrieval in language comprehension. Language and Cognitive Processes, 26(3), 376-405. doi:10.1080/01690965.2010.492642

Hothorn, T., Hornik, K., van de Wiel, M. A., \& Zeileis, A. (2006). A Lego system of conditional inference. The American Statistician, 60(3), 257-263.

Hothorn, T., Hornik, K., \& Zeileis, A. (2006). Unbiased recursive partitioning: A conditional inference framework. Journal of Computational and Graphical Statistics, 15(3), 651674.

Jarema, G. (2006). Compound representation and processing: A cross-language perspective. In G. Libben \& G. Jarema (Eds.), The representation and processing of compound words (pp. 45-70). Oxford: Oxford University Press.

Jespersen, O. (1918). Chapters on English. London: George Allen \& Unwin. Retrieved from http://www.archive.org/stream/chaptersonenglis00jesprich

Jin, M.-J. (2005). English blends: A descriptive study of their distributional patterns and prosodic features. The Modern Linguistic Society of Korea, 20(3), 195-231.

Johnson-Laird, P. N. (1987). The mental representation of the meaning of words. Cognition, $25,189-211$. 
Jones, D. (2011). Cambridge English pronouncing dictionary. (P. Roach, J. Setter, \& J. Esling, Eds.) (18th ed.). Cambridge: Cambridge University Press.

Kaunisto, M. (2000). Relations and proportions in the formation of blend words. Presented at the International Quantitative Linguistic Conference (QUALICO) 2000, Prague.

Kelly, M. H. (1998). To "brunch" or to "brench": Some aspects of blend structure. Linguistics, 36, 579-90.

Kelly, M. H., Bock, J. K., \& Keil, F. C. (1986). Prototypicality in a linguistic context: Effects on sentence structure. Journal of Memory and Language, 25, 59-74.

Kemmer, S. (2003). Schemas and lexical blends. In H. Cuyckens, T. Berg, R. Dirven, \& K.U. Panther (Eds.), Motivation in language: From case grammar to cognitive linguistics. A Festschrift for Gunter Radden (pp. 69-97). Amsterdam \& Philadelphia: Benjamins.

Kubozono, H. (1989). Syntactic and rhythmic effects on downstep in Japanese. Phonology, 6, $39-67$.

Kubozono, H. (1990). Phonological constraints on blending in English as a case for phonology-morphology interface. Yearbook of Morphology, (3), 1-20.

Langacker, R. W. (1987). Foundations of cognitive grammar (Vol. 1). Stanford, CA: Stanford University Press.

Langacker, R. W. (2000). A dynamic usage-based model. In M. Barlow \& S. Kemmer (Eds.), Usage-based models of language (pp. 1-63). Stanford, CA: CSLI Publications.

Lappe, S. (2007). English Prosodic Morphology. Dordrecht: Springer.

Lehrer, A. (1996). Identifying and interpreting blends: An experimental approach. Cognitive Linguistics, 4(7), 359-390.

Lehrer, A. (1998). Scapes, holics, and thons: The semantics of combining forms. American Speech, 73, 3-28.

Lehrer, A. (2003). Understanding trendy neologisms. Italian Journal of Linguistics. Rivista Di Linguistica, 15(2), 369-382.

Lehrer, A. (2007). Blendalicious. In J. Munat (Ed.), Lexical creativity, texts and contexts: The morphology/stylistic interface (pp. 115-133). Amsterdam \& Philadelphia: Benjamins.

Lehrer, A., \& Veres, C. (2010). Two experiments on processing lexical blends. Presented at the International Conference on Lexical Blending, Lyon.

Левенштейн, В. И. [Levenshtein, V. I.]. (1966). Binary codes capable of correcting deletions, insertions, and reversals. Soviet Physics Doklady, 10, 707-710.

Libben, G. (2006). Why study compound processing? An overview of the issues. In G.

Libben \& G. Jarema (Eds.), The representation and processing of compound words (pp. 1-22). Oxford: Oxford University Press.

Libben, G., Gibson, M., Yoon, Y. B., \& Sandra, D. (2003). Compound fracture: The role of semantic transparency and morphological headedness. Brain and Language, 84, 50 64. 
Libben, G., \& Jarema, G. (Eds.). (2006). The representation and processing of compound words. Oxford: Oxford University Press.

López Rúa, P. (2002). On the structure of acronyms and neighbouring categories: A prototype-based account. English Language and Linguistics, 6(1), 31-60. doi:10.1017/S136067430200103X

López Rúa, P. (2004a). Acronyms \& Co.: A typology of typologies. Estudios Ingleses de La Universidad Complutense, 12, 109-129.

López Rúa, P. (2004b). The categorial continuum of English blends. English Studies, 85(1), 63-76.

López Rúa, P. (2007). Keeping up with the times: Lexical creativity in electronic communication. In J. Munat (Ed.), Lexical creativity, texts and contexts (pp. 137159). Amsterdam \& Philadelfia: Benjamins.

Luzzatti, C., Mondini, S., \& Semenza, C. (2001). Lexical representation and processing of morphologically complex words: Evidence from the reading performance of an Italian agrammatic patient. Brain and Language, 79, 345-359. doi:10.1006/brln.2001.2475

MacKay, D. G. (1982). The structure of words and syllables: Evidence from errors in speech. Cognitive Psychology, 3, 210-227.

MacWhinney, B. (2000). Connectionism and language learning. In M. Barlow \& S. Kemmer (Eds.), Usage-based models of language (pp. 121-149). Stanford, CA: CSLI Publications.

Marchand, H. (1960). The categories and types of present-day English word-formation: A synchronic-diachronic approach. Wiesbaden: Otto Harrasowitz.

Marchand, H. (1969). The categories and types of present-day English word-formation: A synchronic-diachronic approach (2nd ed.). München: Beck.

Marslen-Wilson, W. D. (1987). Functional parallelism in spoken word-recognition. Cognition, 25(1-2), 71-102. doi:10.1016/0010-0277(87)90005-9

Marslen-Wilson, W. D., Tyler, L. K., Waksler, R., \& Older, L. (1994). Morphology and meaning in the English mental lexicon. Psychological Review, 101, 3-33.

Marslen-Wilson, W. D., \& Welsh, A. (1978). Processing interactions and lexical access during word recognition in continuous speech. Cognitive Psychology, 10(1), 29-63.

Marslen-Wilson, W. D., \& Zwitserlood, P. (1989). Accessing spoken words: The importance of word onsets. Journal of Experimental Psychology: Human Perception and Performance, 15(3), 576-585.

Mattiello, E. (2013). Extra-grammatical morphology in English: Abbreviations, blends, reduplicatives, and related phenomena. Berlin: Mouton de Gruyter.

McFedries, P. (2011). On-call-ogist. Word spy: The word lover's guide to new words. Retrieved from http://www.wordspy.com/words/on-call-ogist.asp

Meringer, R., \& Mayer, C. (1978). Versprechen und Verlessen: Eine psychologischlinguistische Studie. Amsterdam: Benjamins. 
Mines, M. A., Hanson, B. F., \& Shoup, J. E. (1978). Frequency of occurrence of phonemes in conversational English. Language and Speech, 21(3), 221-241.

Mondini, S., Jarema, G., Luzzatti, C., Burani, C., \& Semenza, C. (2002). Why is "red cross" different from "yellow cross"? A neuropsychological study of noun-adjective agreement within Italian compounds. Brain and Language, 81, 621-634.

Moore, B. C. J., Tyler, L. K., \& Marslen-Wilson, W. D. (Eds.). (2009). The perception of speech: From sound to meaning. Oxford: Oxford University Press.

Myers, J. (2006). Processing Chinese compounds: A survey of the literature. In G. Libben \& G. Jarema (Eds.), The representation and processing of compound words (pp. 169196). Oxford: Oxford University Press.

Neely, J. H. (1991). Semantic priming in visual word recognition: A selective review of current theories and findings. In D. Besner \& G. W. Humphries (Eds.), Basic processes in reading: Visual word recognition (pp. 264-336). Hillsdale, NJ: Erlbaum.

OED Online. Oxford University Press. Retrieved from http://www.oed.com/

Pierrehumbert, J. B. (2001). Exemplar dynamics: Word frequency, lenition and contrast. In J. Bybee \& P. Hopper (Eds.), Frequency and the emergence of linguistic structure (pp. 137-158). Amsterdam: Benjamins.

Piñeros, C.-E. (2004). The creation of portmanteaus in the extragrammatical morphology of Spanish. Probus, 16(2), 203-240.

Pinker, S. (1999). Words and rules: The ingredients of language. New York: Basic Books.

Plag, I. (2003). Word-formation in English. Cambridge: Cambridge University Press.

Plag, I. (2006). Productivity. In B. Aarts \& A. McMahon (Eds.), The handbook of English linguistics (pp. 537-556). Oxford: Blackwell.

Pound, L. (1967). Blends: Their relation to English word formation. Amsterdam: Swets \& Zeitlinger. Reprint: Heidelberg: Winter.

R Development Core Team. (2012). R: A language and environment for statistical computing. $R$ foundation for statistical computing. Vienna. Retrieved from http://www.Rproject.org/

Rastle, K., Davis, M. H., Marslen-Wilson, W. D., \& Tyler, L. K. (2000). Morphological and semantic effects in visual word recognition: A time-course study. Language and Cognitive Processes, 15(4-5), 507-537.

Rastle, K., Harrington, J., Coltheart, M., \& Thomas, B. (2002). 358,534 nonwords: The ARC Nonword Database. Quarterly Journal of Experimental Psychology, 55A, 1339-1362.

Renner, V. (2006). Les composés coordinatifs en anglais contemporain ( $\mathrm{PhD}$ thesis). Université Lumière-Lyon 2, Lyon.

Renner, V. (2008). On the semantics of English coordinate compounds. English Studies, $89(5), 606-613$.

Renner, V., Maniez, F., \& Arnaud, P. J. L. (Eds.). (2012). Cross-disciplinary perspectives on lexical blending. Berlin: De Gruyter Mouton. 
Sandra, D. (1990). On the representation and processing of compound words: Automatic access to constituent morphemes does not occur. Quarterly Journal of Experimental Psychology, 42A, 529-567.

Scalise, S., \& Bisetto, A. (2009). The classification of compounds. In R. Lieber \& P. Štekauer (Eds.), The Oxford handbook of compounding (pp. 34-53). Oxford: Oxford University Press.

Scaltritti, M., \& Balota, D. A. (2013). Are all letters really processed equally and in parallel? Further evidence of a robust first letter advantage. Acta Psychologica, 144(2), 397410. doi:10.1016/j.actpsy.2013.07.018

Schneider, W., Eschman, A., \& Zuccolotto, A. (2002). E-Prime reference guide. Pittsburgh: Psychology Software Tools, Inc.

Schreuder, R., \& Baayen, H. R. (1995). Modeling morphological processing. In L. B. Feldman (Ed.), Morphological aspects of language processing (pp. 131-154). Hillsdale, NJ \& Hove, UK: Laurence Erlbaum Associates.

Semenza, C., \& Mondini, S. (2006). The neuropsychology of compound words. In G. Libben \& G. Jarema (Eds.), The representation and processing of compound words (pp. 7195). Oxford: Oxford University Press.

Shaw, K. E., White, A. M., Moreton, E., \& Monrose, F. (2014). Emergent faithfulness to morphological and semantic heads in lexical blends. In Proceedings of Phonology 2013. The Linguistic Society of America. Retrieved from http://journals.linguisticsociety.org/proceedings/index.php/amphonology/article/view/ 45

Štekauer, P., Valera, S., \& Körtvélyessy, L. (2012). Word-formation in the world's languages: A typological survey. Cambridge: Cambridge University Press.

Sweet, H. (1892). A new English grammar, logical and historical (Vol. 1). Retrieved from http://www.archive.org/details/newenglishgramma100sweeuoft

Taft, M. (2004). Morphological decomposition and the reverse base frequency effect. Quarterly Journal of Experimental Psychology, 57, 745-765.

Taft, M., \& Forster, K. I. (1975). Lexical storage and retrieval of prefixed words. Journal of Verbal Learning and Verbal Behavior, 14, 638-647.

Thurner, D. (1993). Portmanteau dictionary: Blend words in English language, including trademarks and brand names. Ann Arbor, MI: McFarland \& Co.

Tomaszewicz, E. (2012). Output-to-output faithfulness in the phonological structure of English blends. In V. Renner, F. Maniez, \& P. J. L. Arnaud (Eds.), Cross-disciplinary perspectives on lexical blending (pp. 213-232). Berlin: De Gruyter Mouton.

Trommer, J., \& Zimmermann, E. (2012). Portmanteaus as generalized templates. In V. Renner, F. Maniez, \& P. J. L. Arnaud (Eds.), Cross-disciplinary perspectives on lexical blending (pp. 233-258). Berlin: De Gruyter Mouton.

Turner, M., \& Fauconnier, G. (1995). Conceptual integration and formal expression. Metaphor \& Symbolic Activity, 10(3), 183-204. 
Tyler, L. K. (1984). The structure of the initial cohort: Evidence from gating. Perception \& Psychophysics, 36, 217-222.

Van Orden, G. C. (1987). A rows is a rose: Spelling, sound and reading. Memory and Cognition, 15, 181-198.

Wälchli, B. (2005). Co-compounds and natural coordination. Oxford: Oxford University Press.

Warren, B. (1990). The importance of combining forms. In W. U. Dressler, H. Luschützky, O. E. Pfeiffer, \& J. R. Rennison (Eds.), Contemporary morphology (pp. 111-132). Berlin: Walter de Gruyter.

Warren, P. (2013). Introducing psycholinguistics. Cambridge: Cambridge University Press.

Weekes, B. S. (1997). Differential effects of number of letters on word and nonword naming latency. Quarterly Journal of Experimental Psychology, 50(2), 439-456.

Weisberg, S. (2005). Applied linear regression. Hoboken, NJ: Wiley.

Wells, J. C. (1997). SAMPA computer readable phonetic alphabet. In D. Gibbon, R. Moore, \& R. Winski (Eds.), Handbook of standards and resources for spoken language systems (pp. 684-732). Berlin \& New York: Mouton de Gruyter.

Wentworth, H. (1934). Blend-words in English (PhD thesis). Cornell University.

White, S. J., Johnson, R. L., Liversedge, S. P., \& Rayner, K. (2008). Eye movements when reading transposed text: The importance of word-beginning letters. Journal of Experimental Psychology. Human Perception and Performance, 34(5), 1261-1276. doi:10.1037/0096-1523.34.5.1261

Whitney, C. (2001). How the brain encodes the order of letters in a printed word: The SERIOL model and selective literature review. Psychonomic Bulletin and Review, $8(2), 221-243$.

Wood, F. A. (1911). Iteratives, blends, and "streckformen." Modern Philology, 9(2), 157194.

Zwicky, A. M., \& Pullum, G. K. (1987). Plain morphology and expressive morphology. In J. Aske, N. Beery, L. Michaelis, \& H. Filip (Eds.), Proceedings of the 13th Annual Meeting of the Berkeley Linguistic Society (pp. 330-340). Berkeley, CA: Berkeley Linguistic Society. 
Appendix 1. Lexical data

\begin{tabular}{|c|c|c|c|c|c|}
\hline & Type & SW1 & SW2 & SW3 & SW4 \\
\hline abstakiss & $\mathrm{AW}$ & abstain & kiss & & \\
\hline acabowl & AW & academic & bowl & & \\
\hline acatramp & $\mathrm{AC}$ & academic & trampoline & & \\
\hline accountabilibuddy & AW & accountability & buddy & & \\
\hline adorapresh & $\mathrm{AC}$ & adorable & precious & & \\
\hline adverblasting & AW & advertisement & blasting & & \\
\hline advergame & AW & advertisement & game & & \\
\hline adverteasement & infix & advertisement & tease & & \\
\hline adwonderize & infix & advertise & wonderful & & \\
\hline afflufemza & ACEH & affluent & feminist & mother & influenza \\
\hline agapeople & WW & Agape & people & & \\
\hline agflation & $\mathrm{AD}$ & agriculture & inflation & & \\
\hline aggressocracy & $\mathrm{AD}$ & aggressive & aristocracy & & \\
\hline AgOptions & AW & agricultural & options & & \\
\hline agriflation & $\mathrm{AD}$ & agriculture & inflation & & \\
\hline AgSpec & $\mathrm{AC}$ & agricultural & specialists & & \\
\hline aireoke & $\mathrm{AD}$ & air guitar & karaoke & & \\
\hline alcoholimia & WD & alcohol & bulimia & & \\
\hline alculate & $\mathrm{AD}$ & alcohol & calculate & & \\
\hline alternawhore & AW & alternative & whore & & \\
\hline Amazonukkah & WD & Amazon & Hannukkah & & \\
\hline angeurysm & $\mathrm{AD}$ & anger & aneurysm & & \\
\hline approximeeting & AW & approximate & meeting & & \\
\hline assne & WD & ass & acne & & \\
\hline awesmazing & $\mathrm{AD}$ & awesome & amazing & & \\
\hline awesome-itude & WD & awesome & magnitude & & \\
\hline awkfest & AW & awkward & fest & & \\
\hline baggravation & WW & bag & aggravation & & \\
\hline balloonicle & WD & balloon & vehicle & & \\
\hline banquance & $\mathrm{AD}$ & banquet & dance & & \\
\hline Baptlic & $\mathrm{AD}$ & baptist & catholic & & \\
\hline Baracknophobia & WD & Barack & arachnophobia & & \\
\hline bareoke & WD & bare & karaoke & & \\
\hline barsexual & WD & bar & bisexual & & \\
\hline basticherbator & $\mathrm{ADF}$ & bastard & bitch & masturbator & \\
\hline beardo & WD & beard & weirdo & & \\
\hline bedgasm & WD & bed & orgasm & & \\
\hline belligerati & $\mathrm{AD}$ & belligerent & literati & & \\
\hline berb & acr & be & right & back & \\
\hline bevalanche & $\mathrm{AD}$ & beverage & avalanche & & \\
\hline bewrecked & AW & bereft & wrecked & & \\
\hline bigature & WD & big & miniature & & \\
\hline biz cas & $\mathrm{AC}$ & business & casual & & \\
\hline
\end{tabular}




\begin{tabular}{|c|c|c|c|c|c|}
\hline & Туре & SW1 & SW2 & SW3 & SW4 \\
\hline biz cas fri & ACE & business & casual & Friday & \\
\hline blackistani & WD & black & Pakistani & & \\
\hline blaysted & $\mathrm{AD}$ & blazed & wasted & & \\
\hline bleachorexia & $\mathrm{AD}$ & bleach & anorexia & & \\
\hline blizzaster & $\mathrm{AD}$ & blizzard & disaster & & \\
\hline blogebrity & WD & blog & celebrity & & \\
\hline blogel & WD & blog & novel & & \\
\hline blogfic & WC & blog & fiction & & \\
\hline blogorrhea & WD & blog & diarrhoea & & \\
\hline blovel & $\mathrm{AD}$ & blog & novel & & \\
\hline bobo & $\mathrm{AC}$ & bourgeois & bohemian & & \\
\hline boomburb & WD & boom & suburb & & \\
\hline bordinary & $\mathrm{AD}$ & boring & ordinary & & \\
\hline boytastrophe & WD & boy & catastrophe & & \\
\hline boyzilian & WD & boy & Brazilian & & \\
\hline bragabond & WD & brag & vagabond & & \\
\hline bragplain & WD & brag & complain & & \\
\hline breastaurant & WW & breast & restaurant & & \\
\hline breastimate & WW & breast & estimate & & \\
\hline bresilient & AW & brilliant & resilient & & \\
\hline BRIC & acr & Brazil & Russia & India & China \\
\hline briet & $\mathrm{AD}$ & Bridal & diet & & \\
\hline bromo & WD & bro & homo & & \\
\hline bronado & WD & bro & tornado & & \\
\hline brovember & WD & bro & November & & \\
\hline bug & $\mathrm{AD}$ & Boston terrier & pug & & \\
\hline bullycide & WD & bully & suicide & & \\
\hline bungaloft & AW & bungalow & loft & & \\
\hline burqini & $\mathrm{AD}$ & burqa & bikini & & \\
\hline cakeup & WD & cake & makeup & & \\
\hline camruck & AW & camera & ruck & & \\
\hline cankle & AW & calf & ankle & & \\
\hline carbage & WD & car & garbage & & \\
\hline carborexia & $\mathrm{AD}$ & carbon & anorexia & & \\
\hline carcolepsy & WD & car & narcolepsy & & \\
\hline carsophagus & WD & car & sarcophagus & & \\
\hline carspective & WD & car & perspective & & \\
\hline caruck & WD & car & truck & & \\
\hline celeblog & AW & celebrity & blog & & \\
\hline celebutard & $\mathrm{AD}$ & celebutante & retard & & \\
\hline cellacc & WC & cell & accounting & & \\
\hline Centra-vac & $\mathrm{AC}$ & central & vacuum & & \\
\hline chairdrobe & WD & chair & wardrobe & & \\
\hline Chairigami & WD & chair & origami & & \\
\hline
\end{tabular}




\begin{tabular}{|c|c|c|c|c|c|}
\hline & Type & SW1 & SW2 & SW3 & SW4 \\
\hline chalko & WD & chalk & typo & & \\
\hline cheapuccino & WD & cheap & cappuccino & & \\
\hline Chindonesia & ACW & China & India & Indonesia & \\
\hline chmilk & AW & chocolate & milk & & \\
\hline chofa & $\mathrm{AD}$ & chair & sofa & & \\
\hline Christmahannukwanzadan & ACEH & Christmas & Hannukkah & Kwanzaa & Ramadan \\
\hline Christmasochist & WW & Christmas & masochist & & \\
\hline chronoptimist & AW & chronos & optimist & & \\
\hline chugger & $\mathrm{AD}$ & charity & mugger & & \\
\hline cinematard & WD & cinema & retard & & \\
\hline civionics & $\mathrm{AD}$ & civil & electronics & & \\
\hline clapathy & WW & clap & apathy & & \\
\hline classitorium & $\mathrm{AD}$ & classroom & auditorium & & \\
\hline clickmas & WD & click & Christmas & & \\
\hline cockblocalypse & WD & cockblock & apocalypse & & \\
\hline collabulary & $\mathrm{AD}$ & collaborative & vocabulary & & \\
\hline collelephant & AW & college & elephant & & \\
\hline Colocaliexas & $\mathrm{ACF}$ & Colorado & California & Texas & \\
\hline commercide & $\mathrm{AD}$ & commerce & suicide & & \\
\hline communicanine & AW & communication & canine & & \\
\hline complimentsult & WD & compliment & insult & & \\
\hline condomplate & WD & condom & contemplate & & \\
\hline congreenient & infix & convenient & green & & \\
\hline consumerican & $\mathrm{AD}$ & consumerism & American & & \\
\hline consumicane & $\mathrm{AD}$ & consumer & hurricane & & \\
\hline conswervative & infix & conservative & swerve & & \\
\hline converstroyer & $\mathrm{AD}$ & conversation & destroyer & & \\
\hline cookprint & WD & cook & footprint & & \\
\hline copyfighter & AW & copyright & fighter & & \\
\hline cosmolission & $\mathrm{AD}$ & cosmetic & collision & & \\
\hline cowpool & WD & cow & carpool & & \\
\hline crackberry & WD & crack & blackberry & & \\
\hline Crasian & AW & crazy & asian & & \\
\hline creepellent & $\mathrm{AD}$ & creepy & repellent & & \\
\hline croissandwich & AW & croissant & sandwich & & \\
\hline crypster & $\mathrm{AD}$ & crypt & hipster & & \\
\hline dansynch & $\mathrm{AC}$ & dance & synchronise & & \\
\hline datagogy & WD & data & pedagogy & & \\
\hline decorcize & $\mathrm{AD}$ & decorate & exercise & & \\
\hline deleb & $\mathrm{AD}$ & dead & celeb & & \\
\hline delebrity & $\mathrm{AD}$ & designer & celebrity & & \\
\hline Dellionaire & WD & Dell & billionaire & & \\
\hline depinion & $\mathrm{AD}$ & depend & opinion & & \\
\hline diffe & $\mathrm{AC}$ & differential & equation & & \\
\hline
\end{tabular}




\begin{tabular}{|c|c|c|c|}
\hline & Type & SW1 & SW2 \\
\hline digifeiter & $\mathrm{AD}$ & digital & counterfeiter \\
\hline diginecker & $\mathrm{AD}$ & digital & rubbernecker \\
\hline digiroid & $\mathrm{AD}$ & digital & Polaroid \\
\hline disasterbate & $\mathrm{AD}$ & disaster & masturbate \\
\hline disinfortainment & $\mathrm{AD}$ & disinformation & entertainment \\
\hline dotcomrade & WW & dotcom & comrade \\
\hline drail & $\mathrm{AD}$ & drunk & email \\
\hline dramality & $\mathrm{AD}$ & drama & reality \\
\hline drunchie & $\mathrm{AD}$ & drunk & munchie \\
\hline duail & $\mathrm{AD}$ & duck & quail \\
\hline dudevorce & WD & dude & divorce \\
\hline dweet & $\mathrm{AD}$ & drink & tweet \\
\hline ebanatic & $\mathrm{AD}$ & eBay & fanatic \\
\hline econnoisseur & AW & economy & connoisseur \\
\hline edupunk & AW & education & punk \\
\hline elderburb & $\mathrm{AD}$ & elderly & suburb \\
\hline elecoustic & $\mathrm{AD}$ & electric & acoustic \\
\hline elephact & $\mathrm{AD}$ & elephant & fact \\
\hline e-linquent & $\mathrm{AD}$ & $\mathrm{e}$ & delinquent \\
\hline emberrorist & $\mathrm{AD}$ & embarrass & terrorist \\
\hline engayed & infix & engaged & gay \\
\hline engayged & infix & engaged & gay \\
\hline enterdrainment & infix & entertainment & drain \\
\hline enviclean & AW & environment & clean \\
\hline epiphanot & AW & epiphany & not \\
\hline evangellyfish & $\mathrm{AD}$ & evangelical & jellyfish \\
\hline excitipated & infix & excited & anticipate \\
\hline fabject & $\mathrm{AD}$ & fabricated & object \\
\hline fabulash & AW & fabulous & lash \\
\hline Facebookemon & WD & Facebook & pokemon \\
\hline Facebrag & AW & Facebook & brag \\
\hline facehack & AW & Facebook & hack \\
\hline fake-ation & WD & fake & vacation \\
\hline fakeaway & WD & fake & takeaway \\
\hline falk & $\mathrm{AD}$ & Facebook & stalk \\
\hline falloween & WD & fall & halloween \\
\hline fasturbate & WD & fast & masturbate \\
\hline fatimal & WD & fat & animal \\
\hline fauxbia & WW & faux & phobia \\
\hline fauxhemian & WD & faux & bohemian \\
\hline fauxllet & WD & faux & mullet \\
\hline fauxpology & WD & faux & apology \\
\hline fearection & WD & fear & erection \\
\hline feetnastics & WD & feet & gymnastics \\
\hline
\end{tabular}


femcho

fibliography

fictomercial

filmanthropy

fin-lit

flabdomen

flashpacker

flext

flog

floordrobe

flotsametrics

flunami

FoCo

focustrate

folksonomy

fomo

foodoir

formasual

framily

frape

fratire

fratitude

fratmosphere

freeconomics

freshomore

Fridance

friendcest

frienvy

frohawk

Fruitomic

funcate

funkcoustic

fursplode

garriage

gaybernation

gayborhood

gdude

gearotica

geekerati

gigantamous

gimongous

glamping

globesity

globfrag
Type SW1

AD female

AD fictional

$\mathrm{AD}$ fiction

WD film

AC financial

WW flab

WD flash

AD flirt

AD fake

WD floor

WW flotsam

WD flu

AC food

WD focus

WD folk

acr fear

WD food

AD formal

AD friends

AW Facebook

WD frat

WW frat

WW frat

WW free

AD freshman

AW Friday

AD friend

AW friend

BD afro

WD fruit

WD fun

WD funk

WD fur

$\mathrm{AD}$ general

WD gay

WD gay

AW Google

WD gear

WD geek

$\mathrm{AD}$ gigantic

$\mathrm{AD}$ giant

AD glamor

AW global

AC globalisation
SW2

SW3

SW4

macho

bibliography

commercial

philanthropy

literacy

abdomen

backpacker

text

blog

wardrobe

metrics

tsunami

court

concentrate

taxonomy

of

missing out

memoir

casual

family

rape

satire

attitude

atmosphere

economics

sophomore

dance

incest

envy

mohawk

atomic

truncate

acoustic

explode

marriage

hibernation

neighborhood

dude

erotica

literati

famous

humongous

camping

obesity

fragmentation 


\begin{tabular}{|c|c|c|c|c|}
\hline & Type & SW1 & SW2 & SW3 \\
\hline godcast & WD & god & podcast & \\
\hline godvertise & WD & $\begin{array}{l}\text { God } \\
\text { golden }\end{array}$ & advertise & \\
\hline goldendoodle & $\mathrm{AD}$ & retriever & labradoodle & \\
\hline googlectual & WD & Google & intellectual & \\
\hline Googleganger & WD & Google & doppelgänger & \\
\hline Googleverse & WD & Google & universe & \\
\hline GooTube & $\mathrm{AD}$ & Google & YouTube & \\
\hline gorno & $\mathrm{AD}$ & gore & porno & \\
\hline graphilator & $\mathrm{AD}$ & graphing & calculator & \\
\hline grouponcierge & $\mathrm{AD}$ & groupon & concierge & \\
\hline groutfiti & WD & grout & graffiti & \\
\hline guitarthritis & WW & guitar & arthritis & \\
\hline guyliner & WW & guy & eyeliner & \\
\hline hackint & WC & hacking & intelligence & \\
\hline halfadem & WD & half & academ & \\
\hline hangry & AW & hungry & angry & \\
\hline haycaytion & WD & hay & vacation & \\
\hline HENRY & acr & high & earner & not \\
\hline he-vage & WD & he & cleavage & \\
\hline hiccurp & $\mathrm{AD}$ & hiccup & burp & \\
\hline hiddick & AW & hideous & dick & \\
\hline himsomnia & WD & him & insomnia & \\
\hline hippene & $\mathrm{AD}$ & hippie & scene & \\
\hline hipstocritical & $\mathrm{AD}$ & hipster & hypocritical & \\
\hline holidrawl & AW & holiday & drawl & \\
\hline homedulgence & WD & home & indulgence & \\
\hline homoblivious & WW & homo & oblivious & \\
\hline homosinuality & infix & homosexuality & $\sin$ & \\
\hline hompany & $\mathrm{AD}$ & home & company & \\
\hline hormotion & $\mathrm{AD}$ & hormone & emotion & \\
\hline horobvioscope & infix & horoscope & obvious & \\
\hline horrocious & $\mathrm{AD}$ & horrendous & atrocious & \\
\hline hotsome & WD & hot & awesome & \\
\hline hurricancel & AW & hurricane & cancel & \\
\hline hyberdate & AW & hybernate & date & \\
\hline hydfrac & $\mathrm{AC}$ & hydraulic & fracturing & \\
\hline hydrail & $\mathrm{AC}$ & hydrogen & railway & \\
\hline hygeclean & AW & hygienic & clean & \\
\hline iggiot & $\mathrm{AD}$ & ignorant & idiot & \\
\hline ignortant & WD & ignore & important & \\
\hline illuminanswer & AW & illuminate & answer & \\
\hline IM & $\mathrm{AC}$ & instant & message & \\
\hline incredidork & AW & incredible & dork & \\
\hline
\end{tabular}


infimany

intaxication

intellidating

interneuter

intextication

invacuate

investomer

ironeous

jazzerina

Jewtheran

jihobbyist

joyage

jumbrella

juvenoia

kirgin

kleptopenia

knee-mail

laborhood

lacostitute

lapcorn

lapcorn

laughgasm

lavacid

liboobrian

literasewer

lizz

locapour

locationship

loligator

lollercaust

loltard

loopular

lubricake

lucrepath

machinima

mactionary

maffluent

magnetricity

malamanteau

mancation

mancession

manolescent

manpanion

manscape
Type SW1

AW infinitely

infix intoxication

AD intelligent

AW internet

infix intoxication

WD in

WD investor

$\mathrm{AD}$ ironic

WD jazz

WD Jew

AW jihad

WD joy

AW jumbo

$\mathrm{AD}$ juvenile

AD kiss

infix kleptomania

WW knee

WD labor

AD Lacoste

WD lap

AD laptop

AD laugh

AW lava

infix librarian

AW literature

AD laugh

AW local

WD location

WD lol

WD lol

WD lol

WD loop

AW lubricate

WD lucre

WD machine

AD Mac

AW mass

WD magnet

AD malapropism

WD man

WD man

WD man

WD man

WD man
SW2

SW3

SW4

many

tax

dating

neuter

text

evacuate

customer

erroneous

ballerina

Lutheran

hobbyist

voyage

umbrella

paranoia

virgin

pen

email

neighborhood

prostitute

popcorn

acorn

orgasm

acid

boob

sewer

fizz

pour

relationship

alligator

holocaust

retard

circular

cake

psychopath

cinema

dictionary

affluent

electricity

portmanteau

vacation

recession

adolescent

companion

landscape 


\begin{tabular}{|c|c|c|c|c|}
\hline & Type & SW1 & SW2 & SW3 \\
\hline manther & WD & man & panther & \\
\hline manufactroversy & $\mathrm{AD}$ & manufactured & controversy & \\
\hline mascary & AW & mascara & scary & \\
\hline masternap & AW & masturbate & nap & \\
\hline meatoes & WD & meat & potatoes & \\
\hline meformer & WD & me & informer & \\
\hline microllege & $\mathrm{AD}$ & micro & college & \\
\hline microwait & AW & microwave & wait & \\
\hline militainment & $\mathrm{AD}$ & military & entertainment & \\
\hline mindcasting & WD & mind & broadcasting & \\
\hline misinfortainment & $\mathrm{AD}$ & misinformation & entertainment & \\
\hline mobisode & $\mathrm{AD}$ & mobile & episode & \\
\hline mockbuster & WD & mock & blockbuster & \\
\hline molestache & $\mathrm{AD}$ & molester & mustache & \\
\hline momoir & WD & mom & memoir & \\
\hline MoSoSo & ACE & mobile & social & software \\
\hline mousturbate & WD & mouse & masturbate & \\
\hline musicgasm & $\mathrm{AD}$ & music & orgasm & \\
\hline musigasm & $\mathrm{AD}$ & music & orgasm & \\
\hline mysterectomy & $\mathrm{AD}$ & mystery & hysterectomy & \\
\hline naycation & WD & nay & vacation & \\
\hline negatude & $\mathrm{AD}$ & negative & attitude & \\
\hline neologasm & $\mathrm{AD}$ & neologism & orgasm & \\
\hline nerdstalgia & WD & nerd & nostalgia & \\
\hline newater & WW & new & water & \\
\hline newpeat & WD & new & repeat & \\
\hline nicotini & WD & nicotine & martini & \\
\hline nontroversy & WD & non & controversy & \\
\hline nonversation & WD & non & conversation & \\
\hline nounjective & WD & noun & adjective & \\
\hline nutritarian & $\mathrm{AD}$ & nutrient & vegetarian & \\
\hline Obamacan & WD & Obama & republican & \\
\hline Obamacon & WC & Obama & conservative & \\
\hline obliviot & $\mathrm{AD}$ & oblivious & idiot & \\
\hline on-call-ogist & WD & on-call & oncologist & \\
\hline pajamahadeen & WD & pajama & Mujahadeen & \\
\hline paradessence & $\mathrm{AD}$ & paradoxical & essence & \\
\hline parahawking & infix & paragliding & hawk & \\
\hline Par-Don & $\mathrm{AD}$ & Paris & London & \\
\hline parentnoia & WD & parent & paranoia & \\
\hline passthought & AW & password & thought & \\
\hline peegasm & WD & pee & orgasm & \\
\hline peegret & WD & pee & regret & \\
\hline peoplerazzi & WD & people & paparazzi & \\
\hline
\end{tabular}




\begin{tabular}{|c|c|c|c|c|c|}
\hline & Type & SW1 & SW2 & SW3 & SW4 \\
\hline PEWS & acr & post & election & withdrawal & syndrome \\
\hline pext & $\mathrm{AD}$ & penis & text & & \\
\hline philanthrocapitalism & AW & philanthropy & capitalism & & \\
\hline phobar & $\mathrm{AD}$ & photoshop & FUBAR & & \\
\hline pickade & $\mathrm{AD}$ & picket & blockade & & \\
\hline piem & WD & pi & poem & & \\
\hline pi-ku & WD & pi & haiku & & \\
\hline pisshap & WD & piss & mishap & & \\
\hline pizzarrhea & WD & pizza & diarrhoea & & \\
\hline podcatching & AW & podcasting & catching & & \\
\hline Podestrian & $\mathrm{BD}$ & iPod & pedestrian & & \\
\hline poorism & WD & poor & tourism & & \\
\hline potrepreneur & WD & pot & entrepreneur & & \\
\hline prebituary & WD & pre & obituary & & \\
\hline precrimination & WD & pre & recrimination & & \\
\hline predictionary & WW & prediction & dictionary & & \\
\hline prescribble & AW & prescribe & scribble & & \\
\hline pretember & $\mathrm{AD}$ & pretend & remember & & \\
\hline previvor & WD & pre & survivor & & \\
\hline prezactly & $\mathrm{AD}$ & precisely & exactly & & \\
\hline pro-ana & $\mathrm{AC}$ & pro & anorexia & & \\
\hline procrastineating & AW & procrastinating & eating & & \\
\hline procrastishower & AW & procrastinate & shower & & \\
\hline procrasturbate & $\mathrm{AD}$ & procrastinate & masturbate & & \\
\hline profastinate & infix & procrastinate & fast & & \\
\hline pro-mia & $\mathrm{AD}$ & pro & bulimia & & \\
\hline promzilla & WD & prom & Godzilla & & \\
\hline prostiboots & AW & prostitute & boots & & \\
\hline prostidude & $\mathrm{AD}$ & prostitute & dude & & \\
\hline protoduct & $\mathrm{AD}$ & prototype & product & & \\
\hline prowebstinate & infix & procrastinate & web & & \\
\hline puggle & WD & pug & beagle & & \\
\hline pupperware & WD & pup & Tupperware & & \\
\hline quck & $\mathrm{AD}$ & quail & duck & & \\
\hline queird & $\mathrm{AD}$ & queer & weird & & \\
\hline rebellionaire & WD & rebellion & millionaire & & \\
\hline recessionista & WD & recession & fashionista & & \\
\hline refuctor & infix & refactor & fuck & & \\
\hline relaxturbate & WD & relax & masturbate & & \\
\hline remembeer & AW & remember & beer & & \\
\hline rendezbooze & AW & rendezvous & booze & & \\
\hline renoviction & $\mathrm{AD}$ & renovation & eviction & & \\
\hline repuberty & AW & republican & puberty & & \\
\hline ridrunkulous & infix & ridiculous & drunk & & \\
\hline
\end{tabular}




\begin{tabular}{|c|c|c|c|c|c|}
\hline & Type & SW1 & SW2 & SW3 & SW4 \\
\hline ringxiety & WD & ring & anxiety & & \\
\hline robo-signer & AW & robot & signer & & \\
\hline rofldile & WD & rofl & crocodile & & \\
\hline rumint & $\mathrm{AC}$ & rumor & intelligence & & \\
\hline sarcastrophe & $\mathrm{AD}$ & sarcasm & apostrophe & & \\
\hline sarcastrophe & $\mathrm{AD}$ & sarcasm & catastrophe & & \\
\hline sargasm & $\mathrm{AD}$ & sarcasm & orgasm & & \\
\hline SARS & acr & severe & acute & respiratory & syndrome \\
\hline scanlate & WD & scan & translate & & \\
\hline scigov & $\mathrm{AC}$ & science & government & & \\
\hline scoratorium & $\mathrm{AD}$ & score & moratorium & & \\
\hline screenior & WD & screen & senior & & \\
\hline screwvenir & WD & screw & souvenir & & \\
\hline sexsomnia & WD & sex & insomnia & & \\
\hline sext & WD & sex & text & & \\
\hline sexuade & WD & sex & persuade & & \\
\hline sharrow & $\mathrm{AD}$ & shared & arrow & & \\
\hline shitfacedbooking & $\mathrm{AD}$ & shitfaced & facebooking & & \\
\hline shoefiti & WD & shoe & graffiti & & \\
\hline shopulent & WW & shop & opulent & & \\
\hline shorteralls & $\mathrm{AD}$ & shorts & overalls & & \\
\hline shress & $\mathrm{AD}$ & shirt & dress & & \\
\hline shyPod & WW & shy & iPod & & \\
\hline sinlaws & WW & $\sin$ & inlaws & & \\
\hline skeezy & $\mathrm{AD}$ & sketchy & sleazy & & \\
\hline skeptimistic & $\mathrm{AD}$ & skeptical & optimistic & & \\
\hline slacktitude & WD & slack & attitude & & \\
\hline slacktivism & WW & slack & activism & & \\
\hline sleeperson & AW & sleepy & person & & \\
\hline slutsident & WD & slut & president & & \\
\hline smellephant & AW & smelly & elephant & & \\
\hline smexting & $\mathrm{AD}$ & smoking & texting & & \\
\hline SMUM & acr & smart & middle-class & uninvolved & mother \\
\hline solastalgia & $\mathrm{AD}$ & solatium & nostalgia & & \\
\hline SoLoMo & ACE & Social & local & mobile & \\
\hline songlifting & WD & song & shoplifting & & \\
\hline spagbol & $\mathrm{AC}$ & spaghetti & bolognese & & \\
\hline speediac & WD & speed & maniac & & \\
\hline spime & $\mathrm{AD}$ & space & time & & \\
\hline spirmitment & $\mathrm{AD}$ & spirit & commitment & & \\
\hline splinternet & $\mathrm{AD}$ & splinter & internet & & \\
\hline splog & $\mathrm{AD}$ & spam & blog & & \\
\hline squintern & WW & squint & intern & & \\
\hline staycation & WD & stay & vacation & & \\
\hline
\end{tabular}




\begin{tabular}{|c|c|c|c|c|}
\hline & Type & SW1 & SW2 & SW3 \\
\hline stoption & WW & stop & option & \\
\hline store d'oeuvre & WW & store & hors d'oeuvre & \\
\hline sudoopoo & ACE & super & dooper & pooper \\
\hline superstistics & $\mathrm{AD}$ & superstition & statistics & \\
\hline surgicalist & $\mathrm{AD}$ & surgical & hospitalist & \\
\hline suspowledge & $\mathrm{AD}$ & suspicion & knowledge & \\
\hline sweaxy & $\mathrm{AD}$ & sweaty & sexy & \\
\hline SWEDOW & acr & Stuff & we & don't \\
\hline Swirllgasm & WD & Swirll & orgasm & \\
\hline tacolada & WD & taco & enchilada & \\
\hline teaffee & WD & tea & coffee & \\
\hline teavangelist & WD & Tea & evangelist & \\
\hline testosterphone & AW & testosterone & phone & \\
\hline Texodus & AW & Texas & exodus & \\
\hline textpectation & WW & text & expectation & \\
\hline textrovert & WW & text & extrovert & \\
\hline Thankshallowistmas & $\mathrm{ACF}$ & Thanksgiving & halloween & Christmas \\
\hline thuggle & $\mathrm{AD}$ & thug & muggle & \\
\hline thumbo & WD & thumb & typo & \\
\hline thumboard & WD & thumb & keyboard & \\
\hline TINO & acr & Tea & in & name \\
\hline TiNo & AW & TiVo & no & \\
\hline Tiratini & $\mathrm{AD}$ & Tiramisu & martini & \\
\hline tonarrow & $\mathrm{AD}$ & tonight & tomorrow & \\
\hline totes awk & $\mathrm{AC}$ & totally & awkward & \\
\hline traffuck & AW & traffic & fuck & \\
\hline trainimals & WD & train & animals & \\
\hline trimultaneous & WD & tri & simultaneous & \\
\hline truthenize & WD & truth & euthanize & \\
\hline tweetup & $\mathrm{AD}$ & twitter & meetup & \\
\hline tweople & $\mathrm{AD}$ & twitter & people & \\
\hline twetiquette & AW & twitter & etiquette & \\
\hline twidget & AW & twitter & widget & \\
\hline twimmolation & AW & twitter & immolation & \\
\hline twiticism & $\mathrm{AD}$ & twitter & criticism & \\
\hline Twitterhea & WD & twitter & diarrhoea & \\
\hline Twitterverse & WD & twitter & universe & \\
\hline twitticism & AW & twitter & witticism & \\
\hline Twittizen & $\mathrm{AD}$ & twitter & citizen & \\
\hline vachaos & AW & vacation & chaos & \\
\hline vaguyna & infix & vagina & guy & \\
\hline VB6 & acr & vegan & before & $\operatorname{six}$ \\
\hline vcast & $\mathrm{AD}$ & video & podcast & \\
\hline V-commerce & AW & voice & commerce & \\
\hline
\end{tabular}




\begin{tabular}{|c|c|c|c|c|}
\hline & Type & SW1 & SW2 & SW3 \\
\hline vegangelical & WD & vegan & evangelical & \\
\hline vegequarian & $\mathrm{AD}$ & vegetarian & aquarian & \\
\hline videocast & WD & video & podcast & \\
\hline virtopsy & $\mathrm{AD}$ & virtual & autopsy & \\
\hline vishing & $\mathrm{AD}$ & voice & fishing & \\
\hline vlog & $\mathrm{AD}$ & video & blog & \\
\hline VODcast & WD & VOD & podcast & \\
\hline voken & $\mathrm{AD}$ & video & token & \\
\hline voluntell & AW & volunteer & tell & \\
\hline voluntourism & AW & volunteer & tourism & \\
\hline vombomb & AW & vomit & bomb & \\
\hline wag & acr & wives & and & girlfriends \\
\hline WAPathy & WW & WAP & apathy & \\
\hline webemy & WD & web & enemy & \\
\hline webtrovert & WD & web & introvert & \\
\hline wedsite & $\mathrm{AD}$ & wedding & website & \\
\hline weisure & $\mathrm{AD}$ & work & leisure & \\
\hline whack & AW & wireless & hack & \\
\hline wifive & WD & wi-fi & high-five & \\
\hline wikiality & $\mathrm{AD}$ & wikipedia & reality & \\
\hline wikillectual & $\mathrm{AD}$ & wikipedia & intellectual & \\
\hline wikiot & $\mathrm{AD}$ & wikipedia & idiot & \\
\hline wlexia & $\mathrm{AD}$ & www & dyslexia & \\
\hline wordanista & WD & word & fashionista & \\
\hline yesterclass & AW & yesterday & class & \\
\hline yestersol & $\mathrm{AC}$ & yesterday & solar & \\
\hline zomburlesque & AW & zombie & burlesque & \\
\hline
\end{tabular}




\section{Appendix 2. Ethics Approval}

IL "ASHE WANAXCA O TE LJUKC O TL IKA A KAL

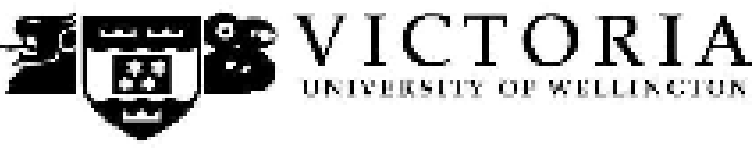

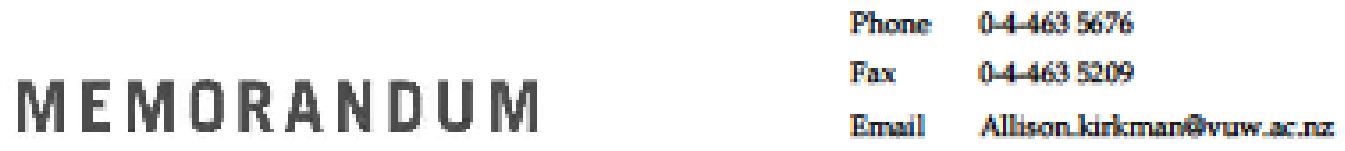

\begin{tabular}{l|l}
\hline TO & Natalia Beliaeva \\
\hline COPY TO & $\begin{array}{l}\text { Laurie Bauer } \\
\text { Paul Warren }\end{array}$ \\
\hline FROM & Dr Kathy Nelson, Acting Convener, Human Ethics Committee \\
\hline \multicolumn{2}{|l|}{23 August 2012} \\
\hline PAGES & 1 \\
\hline SUBJECT & $\begin{array}{l}\text { Ethics Approval: 19393 } \\
\text { English blends among neighbouring word formation } \\
\text { categories: some aspects of their structure }\end{array}$ \\
\hline
\end{tabular}

Thank you for your application for ethical approval, which has now been considered by the Standing Committee of the Human Ethics Committee.

Your application has been approved from the above date and this approval continues until 30 April 2014. If your data collection is not completed by this date you should apply to the Human Ethics Committee for an extension to this approval.

Best wishes with the research.

Kathy Nelson

Human Ethics Committee 


\section{Appendix 3. Information sheet for participants of the web-based}

\section{survey}

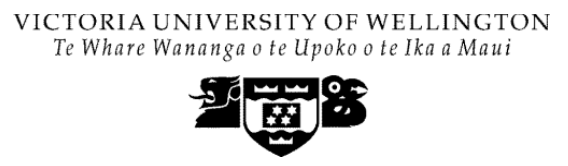

School of Linguistics and Applied Language Studies

PO Box 600, Wellington 6140

tel: 4635600

fax: 4635604

Researcher: Natalia Beliaeva: natalia.beliaeva@vuw.ac.nz

Supervisors: Laurie Bauer: laurie.bauer@,vuw.ac.nz

Paul Warren: paul.warren@vuw.ac.nz

\section{Let's have a word about words!}

I am a PhD student in Linguistics at Victoria University of Wellington undertaking a research project that will lead to a thesis. With this project, I am looking at how people use language and understand new words.

This project has been granted ethical approval by the Victoria University of Wellington Human Ethics Committee.

I am inviting native speakers of English to complete an online questionnaire which will take around 15 minutes. In acknowledgement of your time and effort we will include you in the prize draw for a gift voucher worth $100 \mathrm{NZ}$ dollars.

By following the link below you give your consent for me to use your responses in the research project.

The survey is strictly anonymous. Any personal information, e.g. age or first language, will be collected for statistical reasons only and no identities will be associated with any responses. The collected data will be stored in password protected file for the duration of this study.

The thesis will be submitted for marking to Victoria University of Wellington and deposited in the University Library, after which it will become available electronically. I also intend to publish articles based on the data in scholarly journals. Additionally, I will produce a summary report of the findings of the project for participants which will be sent to you if you make this request by ticking the appropriate box on the survey webpage. The collected data will be destroyed 2 years after the conclusion of the research.

If you have any questions or would like to receive further information about the project, feel free to contact me or my supervisors using the details provided above.

\section{To start the survey, follow this link:} http://vuw.qualtrics.com/SE/?SID=SV bBJRbTNWX8CTMod 


\section{Appendix 4. Survey stimuli}

\section{Main survey}

The questions in Part 1 were presented to the participants in a random order, preceded by the instruction that is given below. Each question contained only one of the four definitions of the word in bold type. Part 2 questions and were presented after Part 1, in the same order as in this sample.

\section{Part 1}

Thank you for agreeing to participate in our survey. It should take around 15 minutes to answer all the questions.

You will see a total of 64 sentences displayed successively on 10 pages. After each sentence there is a definition for the word in bold type. Please read the sentences and answer how successfully you think each definition explains the word by choosing the appropriate option.

1. In fact, Ontario may have some catching up to do if it's serious about being a world leader in hydrails. A European consortium called The Hydrogen Train concluded a study last year that looked at what it would take to demonstrate a hydrogen train in Denmark by 2010.

\section{hydrail -}

1) hydrogen railway

2) trains that use hydrogen fuel

3) a railway system that uses highly flammable gas fuel

4) trains that use highly flammable gas fuel

2. I am a scigov double major. scigov -

1) science and government studies

2) science and politics studies

3) natural and government studies

4) natural and politics studies

3. Bobos talk like hippies but walk like yuppies, decrying materialism while indulging in all manner of luxuries. bobo -

1) a bourgeois bohemian

2) a socially unconventional bourgeois person

3) a person who likes both a bohemian and a luxurious lifestyle

4) a socially unconventional person who likes a luxurious lifestyle

4. For 18 year Consolidated Credit Counseling Services focused on educating youth finlit about money management and finlit topics.

1) financial literacy

2) the ability to understand financial texts

3) literacy in the field of money and economics

4) the ability to understand texts related to money and economics 
5. He says, and others back him up, that the Pentagon took dubious accounts from émigrés close to Ibrahim Zuhair and gave these tales credibility they did not deserve. Intelligence analysts ... refer contemptuously to recent work as "rumint"...

rumint -

1) rumour intelligence

2) sophisticated information based on rumours rather than facts

3) intelligence information from unreliable sources

4) sophisticated information from unreliable sources

6. You only need to look into a person's bedroom to realize the seriousness of globfrag. He is stuck on his computer 24/7 with instant knowledge from all over the globe at his fingertips, yet does not know what his family is up to.

globfrag -

1) globalisation and simultaneous fragmentation

2) globalisation and simultaneous breakdown in connections between people

3) international integration and simultaneous fragmentation

4) international integration and simultaneous breakdown in connections between people

7. Someone lounges lazily on the Acatramp, ... diligent studiers read on chairs outside as the stacks blare, groups cluster on the fourth floor balcony, and a Frisbee whizzes past my ear: it's Friday afternoon.

\section{acatramp -}
1) an academic trampoline
2) an academic bouncing device
3) a trampoline for students and professors
4) a bouncing device for students and professors

8. "Oh my goodness, Michael gave me the most beautiful bouquet for our six-month anniversary today." - "Aww, that's adorapresh!"

\section{adorapresh -}
1) adorable and precious
2) adorable and valuable
3) enchanting and precious
4) enchanting and valuable

\section{biz cas -}

9. Whip out your best biz cas for this event.
1) business casual clothing
2) business informal clothing
3) workplace casual clothing
4) workplace informal clothing

\section{IM me later}

IM -
1) instant message
2) instant communication
3) real-time online message
4) real-time online communication 
11. When she heard music coming over the grocery store's PA system, Amie started to dansynch until the music was over.

\section{dansynch -}

1) dance in synchronization with the music

2) dance in time with the music

3) move rhythmically in synchronization with the music

4) move rhythmically in time with the music

12. Diffe is the hardest and perhaps most widely taken math class at our university. diffe -

1) differential equations

2) mathematical statements involving differentials

3) equations involving derivatives of functions

4) mathematical statements involving derivatives of functions

13. Do you want to eat at the FoCo tonight?

FoCo -

1) a food court

2) an area with food outlets

3) a snack bar and restaurant court

4) an area with snack bars and restaurants

14. With the rushed passage into law of the Digital Economy Act this month, the fight over copyright enters a new phase. Previous to this, most copyfighters operated under the rubric that a negotiated peace was possible between the thrashing entertainment giants and civil society.

\section{copyfighter -}

1) a copyright fighter

2) a person who protests against copyright

3) a person who fights against legal control over created work

4) a person who protests against legal control over created work

15. The journalistic celeblog has its roots in fertile soil - the hallowed tradition of "Nobody asked me, but..." columns that newspaper hacks have for decades used to fill space with short-paragraph rambles on the folly of Liberals, the folly of Conservatives, or the folly of the guy who invented the shrink wrap on compact disks.

\section{celeblog -}

1) a blog written by or about a celebrity

2) a web journal written by or about a celebrity

3) a blog written by or about a famous person

4) a web journal written by or about a famous person

16. A growing number of tourists - especially aging Baby Boomers and students - are keen to explore some of the darkest corners of the world, and maybe even work there for a while on "voluntourism" projects.

\section{voluntourism -}

1) volunteer tourism

2) volunteering while travelling

3) tourism that involves doing unpaid work 
4) doing unpaid work while travelling

17. But this kind of flexibility - we call it approximeeting - can also engender a new sense of insecurity.

approximeeting -

1) an approximate meeting arrangement

2) an approximate appointment arrangement

3) a meeting arrangement with no precise details specified

4) an appointment arrangement with no precise details specified

18. While people may be tricked into giving up their passwords, smartcards may be lost or stolen, as can biometric templates stored on computers for comparing eye or fingerprint scans, so-called "passthoughts" are unique.

\section{passthought -}

1) a thought pattern used as a password

2) a brain signal used as a password

3) a thought pattern giving access to a computer system

4) a brain signal giving access to a computer system

19. Most of these will be redevelopments into what some people call "bungalofts" houses bought for about $\$ 180,000$ by young professionals and modernized ... with clear sight lines to the backyard and custom millwork.

\section{bungaloft -}

$$
\text { with clear sight lines to the backyard and custom millwork. }
$$

1) a bungalow with a loft

2) a bungalow with an attic

3) a detached low-rise house with a loft

4) a detached low-rise house with an attic

20. Officials say advergames promote repeat traffic to Web sites and reinforce brands in compelling ways. Because users choose to register to be eligible for prizes, the games help marketers collect customer data.

\section{advergames -}

1) games that incorporate advertisement

2) entertainment programmes that incorporate advertisement

3) games that incorporate commercial announcements

4) entertainment programmes that incorporate commercial announcements

21. Where is that steak I ordered? We've been waiting for an hour and a half here. The service here is terrible! I'm starving! I don't know about you, but I'm starting hangry to feel really hangry!

1) hungry and angry

2) hungry and strongly displeased

3) feeling need for food and angry

4) feeling need for food and strongly displeased

22. Hey, did you see Jane's newest Facebrag? Eesh.

\section{Facebrag -}

1) Facebook bragging

2) Facebook boasting 
3) bragging in a social network

4) boasting in a social network

23. Crammer News uses the opinions of politiclones to bolster support for the President's Administration.

\section{politiclones -}

1) people who clone their political opinions from others

2) people who copy their political opinions from others

3) people who clone their opinions about governmental actions from others

4) people who copy their opinions about governmental actions from others

24. Jackie was filled with frienvy as she walked through the halls with her best friend Tracey, because all of the guys were complimenting Tracey on her hot new look.

\section{frienvy -}

1) envy of a friend

2) desire for something that a friend might have

3) envy of a close acquaintance

4) desire for something that a close acquaintance might have

25. Try eating our new Croissandwich!

croissandwich -

1) the hybrid of croissant and sandwich

2) a croissant which is sliced and filled

3) a sandwich made of a crescent shaped bread roll

4) a sliced and filled crescent shaped bread roll

\section{Classes are hurricancelled!}

\section{hurricancelled -}

1) cancelled because of a hurricane

2) called off because of a hurricane

3) cancelled because of a severe storm

4) called off because of a severe storm

27. The Twitter-based application, twhirl, is an example of a handy twidget. twidget -

1) a Twitter widget

2) a small software application for Twitter

3) a widget for a microblogging service

4) a small software application for a microblogging service

28. Call it the courtship equivalent to the slow-food movement. Call it a backlash against point and click matchmaking. Whichever, intellidating - an unhurried, decidedly highbrow approach to mating - is catching on in Boston, New York, Toronto and beyond...

\section{intellidating -}

1) dating that emphasizes intelligence

2) romantic relationships that emphasize intelligence

3) dating that involves mentally stimulating activity

4) romantic relationships that involve mentally stimulating activity 
29. "Investomers", or people who both hold stock in a particular company and buy products from that company, are generally more loyal and more profitable than investomer their nonequity-owning counterparts.

1) a customer who is also an investor

2) a client who is also an investor

3) a customer who is also a shareholder

4) a client who is also a shareholder

30. So, after a year of trying to figure out what I was going to do, I decided to quit my jazzerina ballet life and become a "Jazzerina!"

1) a ballerina who performs a jazz dance

2) a classical dancer who performs a jazz dance

3) a ballerina who performs an improvisation dance to syncopated music

4) a classical dancer who performs an improvisation dance to syncopated music

31. "Those pants don't make your butt look so big" - that's a complimentsult! complimentsult -

1) a compliment which is also an insult

2) a compliment which is also offensive

3) praise which is also an insult

4) praise which is also offensive

32. The move to "homedulgence" is one way consumers can ride out the recession and it is predicted it will soon extend to many other areas of life, such as mixyour-own cocktails evenings and home dining clubs.

\section{homedulgence -}

1) home-based indulgence

2) home-based entertainment

3) indulgence without going out

4) entertainment without going out

33. Shoppers trying to survive the credit crunch are also spending less on takeaways and making home-made "fakeaways" of their favourite dishes.

\section{fakeaway -}

1) a fake takeaway

2) a fake restaurant meal

3) a homemade meal similar to takeaway

4) a homemade meal similar to one made in a restaurant

34. Uh, you might want to get rid of all this carbage before you pick up your date tonight.

\section{carbage -}

1) garbage in one's car

2) trash in one's car

3) garbage in one's vehicle

4) trash in one's vehicle

35. The deluded women who bought Boyzilians as gifts for their boyfriends need a reality check. 


\section{boyzilian -}

1) Brazilian waxing for boys

2) bikini waxing for boys

3) Brazilian waxing for men

4) bikini waxing for men

36. Look up around your city and what do you see? A bird? A plane? No, it's a pair of mangy sneakers dangling from the powerlines. Shoe-flinging, or shoefiti, has emerged as one of the more inexplicable forms of cultural expression in innercity Melbourne.

shoefiti -

1) graffiti made of shoes

2) street decoration made of shoes

3) graffiti made of footwear

4) street decoration made of footwear

37. "I want all viewers to feel a part of this mission." Some might liken this type of documentary and fundraising event to the trend toward "filmanthrophy."

\section{filmanthropy -}

1) making films for philanthropic reasons

2) making films to raise money for charity

3) moviemaking for philanthropic reasons

4) moviemaking to raise money for charity

38. Many of us will be familiar with the basest form of ringxiety - when one phone rings and everyone in the vicinity suddenly starts checking their pockets or handbags with frantic abandon.

\section{ringxiety -}

1) anxiety caused by telephone ringing

2) uneasiness caused by telephone ringing

3) anxiety caused by the sound of a telephone call

4) uneasiness caused by the sound of a telephone call

39. Just as we continue to educate fans about the right ways to enjoy music online, we will continue to enforce our rights through the legal system. Songlifting is not without consequences.

\section{songlifting -}

1) online shoplifting of songs

2) unauthorised downloading of songs

3) online shoplifting of musical copyright material

4) unauthorised downloading of musical copyright material

40. Of the 68 miniature sets and models in The Lord Of The Rings, about 30 were dubbed "bigatures" because they were so large and detailed, people could have

\section{bigature -} actually lived in them.

\section{1) a big miniature}

2) a big, rather than small, replica or model

3) a large scale model rather than miniature

4) a large scale, rather than small, replica or model 
41. "Man, she was on Facebook flirting with everyone and talking about all her wild parties, but when we met in person she just stared at the floor and mumbled everytime I asked her something." - "Yeah, sounds like she's kind of a webtrovert. "

webtrovert -

1) a web extrovert

2) a person who is socially confident and outgoing on the web

3) an internet extrovert

4) a person who is socially confident and outgoing on the internet

42. Tagging often produces strange, overlapping characterizations with surprisingly beneficial results. Some have called the results a "folksonomy."

\section{folksonomy -}

1) a folk taxonomy

2) a folk classification

3) a taxonomy based on people's opinions

4) a classification based on people's opinions

43. This is where I work with other people to scanlate manga. You're welcome to help, no commitment needed.

\section{scanlate -}

1) scan and translate

2) scan and convert into another language

3) convert into digital form and translate

4) convert into digital form and into another language

44. Magazines have taken to publishing before and after shots of "painfully thin Kate", calling her "Queen of diets" and "Slimline Kate", and speculating on the "briet".

briet -

1) a diet for brides

2) a special course of food for brides

3) a diet for women just before marriage

4) a special course of food for women just before marriage

45. Is 'sceptimistic' the ideal mindset for a strategist? sceptimistic -

1) both sceptical and optimistic

2) both sceptical and confident about the future

3) both doubting and optimistic

4) both doubting and confident about the future

46. Whether you are weathering the storm by building snowmen or plowing through piles of snow on your driveway, we are asking for photos of how you honor Old Man Winter. And if you want to stay in the comfort of your home, just post a

\section{blizzaster -} photo of your backyard blizzaster.

1) a disaster caused by a blizzard

2) a sudden accident caused by a blizzard

3) a disaster caused by a snow storm

4) a sudden accident caused by a snow storm 
47. 'Products blessed with paradessence somehow combine two mutually exclusive states and satisfy both simultaneously. Ice cream melds eroticism and innocence. Air travel offers sanitised adventure. Amusement parks provide terror and reassurance. Automobiles render drivers reckless and safe.

paradessence -

1) a paradoxical essence

2) a paradoxical characteristic

3) a self-contradictory essence

4) a self-contradictory characteristic

48. Weisure has been fuelled by social networking sites like Facebook and MySpace, where "friends" may actually be business partners or work colleagues. weisure -

1) a combination of work and leisure

2) a combination of business-related tasks and leisure

3) a combination of work and free time

4) a combination of business-related tasks and free time

49. While the IFA is protesting - and not "pickading" any of the plants - farmers will not deliver animals across those protest lines. While some factories have their own supplies, these are estimated to be less than a fortnight's kill. With relations between the two sides deteriorating, "pickades" could well be the next step.

pickade -

1) a picket which is also a blockade

2) a picket which prevents goods or people from entering or leaving a place

3) people gathering outside a building to protest and to make a blockade

4) people gathering at a place to protest and to prevent goods or people from entering or leaving it

50. I am promoting a new protoduct on this website. protoduct -

1) a prototype which is also a product

2) a prototype which is also released for sale

3) a preliminary version of something which is also a product

4) a preliminary version of something which is also released for sale

51. Once out, the tenants are not allowed to renew their leases until they agree to monstrous rent increases, sometimes double what they paid before the renoviction.

\section{renoviction -}

1) renovation of an apartment coupled with the eviction of its tenants

2) renovation of an apartment coupled with the expulsion of its tenants

3) repair works in an apartment coupled with the eviction of its tenants

4) repair works in an apartment coupled with the expulsion of its tenants

52. If I want to share my word tags with a community, I'm contributing to a collabulary. 
1) collaboratively compiled vocabulary

2) collaboratively compiled body of words

3) a vocabulary compiled by several parties working together

4) a body of words compiled by several parties working together

53. Last year chuggers coaxed 700,000 donors into pledging $\$ 240$ million to charities over the next five years. About 100 charities hire chuggers to boost revenues. Although one high-street fundraiser can cost as much as $\$ 100$ a day, charities say they are good value.

\section{chugger -}
1) a charity mugger
2) a person who forces people to donate money for charity
3) a fundraising mugger
4) a person who forces people to donate money for fundraising

54. "No one's allowed in the living room — only adults," says Zalta, flopping down on chofa his favourite piece of furniture, the "chofa".

1) a hybrid of a chair and a sofa

2) a wide and soft chair

3) a small sofa for one person

4) a wide and soft seat for one person

55. "Dramalities," they say, are satiating people's needs for sanitized gossip, Peeping Tomism, and the pathetic desire to feel superior. dramality -

1) a combination of drama and reality

2) a combination of drama and real-life events

3) a combination of role play and reality

4) a combination of role play and real-life events

56. Where once there were objects, now there are well, fabjects.

\section{fabject -}

1) a fabricated object

2) a fabricated thing

3) an object invented in order to deceive

4) a thing invented in order to deceive

57. Lenny's neighbours called the cops to complain about his late night guitar solos, elecoustic so now he has to go elecoustic.

1) an electric guitar used as an acoustic one

2) an electric guitar used unplugged

3) an amplified guitar used as an acoustic guitar

4) an amplified guitar used unplugged

58. Put down your phone and stop flexting.

\section{flexting -}

1) flirting while texting

2) flirting while sending SMS

3) demonstrating playfully amorous behaviour while texting 
4) demonstrating playfully amorous behaviour while sending SMS

59. I really like this blogfic. I plan on continuing to read it until it's abandoned or the blogfic writer brings it to a sensible conclusion.

1) blog fiction

2) blog literature

3) web journal fiction

4) web journal literature

60. According to a US presidential commission, the global population with the computer skills required for hackint operations and other forms of cyber-attack against important Western targets has grown from a few thousand 20 years ago to about 19 million today.

\section{hackint -}

1) hacking intelligence

2) obtaining secret information by hacking

3) unauthorised accessing a computer system to get intelligence

4) obtaining secret information unauthorised accessing a computer system

61. According to stock-tracking firm IP0.com, more than a quarter of the 71 netcos that went public in the past year are now trading under their offering price. netco -

1) an internet company

2) an internet business

3) an online company

4) an online business

62. See the white earphones, she's got to be a podestrian.

\section{podestrian -}

1) a pedestrian with an iPod

2) a person walking on the street with an iPod

3) a pedestrian with a specific portable digital media player

4) a person walking on the street with a specific portable digital media player

63. Sporting a sharp 'frohawk' that day, though, he was not exactly easy to picture in a choir robe.

\section{frohawk -}

1) an afro hairstyle shaped as a mohawk

2) an afro hairstyle in which both sides of the head are shaven

3) a hairstyle with frizzy hair shaped as a mohawk

4) a hairstyle with frizzy hair in which both sides of the head are shaven

64. The largest group of Obamacons hail from the libertarian wing of the movement.

\section{Obamacon -}

1) a Conservative who supports Obama

2) a person who does not support the Democratic party but supports Obama

3) a Conservative who supports the Democratic president

4) a person who does not support the Democratic party but supports the Democratic president 


\section{Part 2}

Please answer the following questions about yourself

1. Are you male or female?

male ; female

2. What is your age?

18-25 . 26-30 ; 31-40 ; 41-50 ; 51-60 ; over 60

3. What is your native language? English ; other (please specify)

4. Have you taken a university course in linguistics? $\mathrm{Y} \quad ; \mathrm{N}$

5. Do you want to take part in the prize draw for a gift voucher worth $100 \mathrm{NZ}$ dollars?

$\mathrm{Y}_{\text {___}}$; $\mathrm{N}_{\text {___ }}$; if yes, please provide your e-mail address:

6. Do you want to receive an e-mail with a report of the research when it is completed?

$\mathrm{Y}$

(the e-mail address will not be associated with your responses and will not be used for any purposes other than prize draw and sending a report of the research).

\section{Additional survey stimuli (with WW target words)}

65. Ebbesmeyer has found that rubber duckies and sneakers lost off container ships have a way of capturing the public's attention. He has used them over the past two decades to illustrate the persistence of plastics and other floating trash in the oceans. Now, along with science writer Eric Scigliano, he has written Flotsametrics and the Floating World: How One Man's Obsession with Runaway Sneakers and Rubber Ducks Revolutionized Ocean Science.

\section{flotsametrics -}

1) a system of metrics using flotsam

2) calculating something using flotsam

3) a system of metrics using wreckage or cargo floating on the sea

4) calculating something using wreckage or cargo floating on the sea

66. In general, you can't fight against your genetic strengths and come off the conqueror. If you're the type of young adult that gets called "cute" and "sweet" over "hot," guyliner isn't likely for you. Eye makeup doesn't tend to make "hot" out of "sweet," but it certainly can make "yikes."

\section{guyliner -}

1) an eyeliner for guys

2) an eye makeup product for guys

3) an eyeliner for men

4) an eye makeup product for men

67. Predictionary is about blank spaces in language and culture and their formative role in conceptual and artistic creativity.

\section{predictionary -}

1) a dictionary containing predictions

2) a reference book containing predictions 
3) a dictionary containing statements about the future

4) a reference book containing statements about the future

68. Michael Robertson says teachers setting up Internet projects underestimate the pleasure people get out of doing something that feels like a public service yet requires no more than a few keystrokes. "It's all fed by slacktivism," he said, "the desire people have to do something good without getting out of their chair."

\section{slacktivism -}

1) slack activism

2) slack efforts to promote or impede changes

3) lazy activism

4) lazy efforts to promote or impede changes

69. Ryan's uncomfortable laughter at the Secret Santa Pot Luck indicated he was a

\section{Christmasochist.}

\section{Christmasochist -}

1) a Christmas masochist

2) a person involved in Christmas activities despite suffering from them

3) a yuletide masochist

4) a person involved in yuletide activities despite suffering from them

70. Because Doug downloaded the new hit "Outrageous" he was totally shyPod when Dru wanted to take a peek at his Library.

\section{ShyPod -}

1) feeling shy about sharing the contents of one's iPod

2) feeling shy about sharing the contents of one's portable media player

3) feeling bashful about sharing the contents of one's iPod

4) feeling bashful about sharing the contents of one's portable media player

71. "That graduation ceremony was so long, I got clapathy!"

\section{clapathy -}

1) apathy caused by excessive clapping

2) indifference caused by excessive clapping

3) apathy caused by excessive applauding

4) indifference caused by excessive applauding

72. Kelly: "So how'd the conversation go with Bill last night?"

Wendy: "Ah he's such a textrovert. We didn't make any progress until I went home and he spilled his guts over texts."

\section{textrovert -}

1) a texting extrovert

2) a person who is socially outgoing while texting

3) an SMS extrovert

4) a person who is socially outgoing while sending SMS

73. "Man my wrists hurt from my guitarthritis" 
1) arthritis caused by playing the guitar

2) inflammation of a joint caused by playing the guitar

3) arthritis caused by playing a musical instrument

4) inflammation of a joint caused by playing a musical instrument

74. "So who's this NrdPowr32 guy?"

"I dunno. Just a dotcomrade of mine."

\section{dotcomrade -}

1) a dotcom comrade

2) a dotcom friend

3) an internet comrade

4) an internet friend

75. "A marvellous beginning to pure shopulence! Our fabulous Weekender opener will include an exclusive wine tasting and fashion preview at a private, VIP venue"

\section{shopulence -}

1) the opulence of a shop

2) the extensive decorations and luxury of a shop

3) the opulence of a place where goods are sold

4) the extensive decorations and luxury of a place where goods are sold

76. I couldn't help but feel baggravation as I watched other passengers get their luggage and leave the airport.

\section{baggravation -}

1) aggravation caused by waiting for bags

2) continuing irritation caused by waiting for bags

3) aggravation caused by waiting for luggage

4) continuing irritation caused by waiting for luggage

77. Customer: Hi there, I would like a bra fitting please.

Shop assistant: OK, well I breastimate you are a 10/32C so let's start there, shall we?

\section{breastimate -}

1) to estimate the size of one's breasts

2) to make a judgement about the size of one's breasts

3) to estimate the size of one's bra cups

4) to make a judgement about the size of one's bra cups

78. "I need to work out, I'm getting a flabdomen."

\section{flabdomen -}

1) a flabby abdomen

2) a flabby belly

3) unwanted body fat on one's abdomen

4) unwanted body fat on one's belly

79. "Dude, I went to this party last night with some of my college buddies and we hit the twostory beer bong just like old times... I'm not usually one to get that wild anymore, but I got sucked into the fratmosphere of the party." 


\section{fratmosphere -}

1) a frat atmoshpere

2) an atmoshpere typical of a college students social club

3) a frat environment

4) an environment typical of a college students social club 


\section{Appendix 5. Item variables used in the survey}

\begin{tabular}{|c|c|}
\hline Variable name & Variable description \\
\hline Length & The number of letters in the blend or clipping compound \\
\hline w1length & The number of letters in $\mathrm{W} 1$ \\
\hline w2length & The number of letters in W2 \\
\hline sp.ini.1length & The number of letters in the initial splinter of $\mathrm{W} 1$ \\
\hline sp.fin.1length & The number of letters in the final splinter of W1 \\
\hline sp.ini.2length & The number of letters in the initial splinter of W2 \\
\hline sp.fin.2length & The number of letters in the final splinter of W2 \\
\hline w1freqCoca & Coca frequency of $\mathrm{W} 1$ \\
\hline w2freqCoca & Coca frequency of W2 \\
\hline BlendFreqGoogle & Google frequency of the blend / clipping compound \\
\hline Blend.type & $\begin{array}{l}\text { Structural type of the target word: AC, AD, AW, WD, WC, BD, BC or } \\
\text { WW }\end{array}$ \\
\hline SemanticType & $\begin{array}{l}\text { Semantic type of the target word: syntagmatic origin (Synt) or } \\
\text { paradigmatic origin (Para) }\end{array}$ \\
\hline w1similarity & $\begin{array}{l}\text { Orthographic similarity of } \mathrm{W} 1 \text { to the target word, i.e. the number of } \\
\text { letters that the target word shares with W1 divided by total number } \\
\text { of letters in W1 }\end{array}$ \\
\hline w2similarity & $\begin{array}{l}\text { Orthographic similarity of W2 to the target word, i.e. the number of } \\
\text { letters that the target word shares with W2 divided by total number } \\
\text { of letters in W2 }\end{array}$ \\
\hline ASED & $\begin{array}{l}\text { Average string edit distance between the source words and the } \\
\text { blend/clipping compound (Levenshtein, 1966)) calculated in the } \\
\text { following way (Gries, 2012): (W1 } \rightarrow \text { blend }+ \text { W2 } \rightarrow \text { blend) / } 2 \text {, i.e. the } \\
\text { average of the number of letters that have to be inserted / deleted / } \\
\text { substituted to turn W1 into the target word plus the number of } \\
\text { insertions / deletions / substitutions that are necessary to turn W2 } \\
\text { into the target word }\end{array}$ \\
\hline ProsW1 & $\begin{array}{l}\text { Whether the target word preserves the prosodic pattern (i.e. the } \\
\text { number of syllables and the main stress position) of W1 }\end{array}$ \\
\hline ProsW2 & $\begin{array}{l}\text { Whether the target word preserves the prosodic pattern (i.e. the } \\
\text { number of syllables and the main stress position) of W2 }\end{array}$ \\
\hline FPW1 & $\begin{array}{l}\text { The frequency position of W1 in COCA among all the words } \\
\text { beginning/ending with the letter string in the splinter, calculated in } \\
\text { the following way: if W1 is the most frequent word in COCA among } \\
\text { all the words beginning with the letter string in the splinter, this } \\
\text { means that its frequency position is } 1 \text {, if it is the second most } \\
\text { frequent word beginning with that letter string, than its frequency } \\
\text { position is } 2 \text {, and so on }\end{array}$ \\
\hline FPW2 & $\begin{array}{l}\text { The frequency position of W2 in COCA among all the words } \\
\text { beginning/ending with the letter string in the splinter }\end{array}$ \\
\hline CoComb & $\begin{array}{l}\text { The COCA frequency of the coordinative combination (with and, or, } \\
\text { or a comma) of the source words of the target word }\end{array}$ \\
\hline SubComb & $\begin{array}{l}\text { The COCA frequency of the subordinative combination of the source } \\
\text { words of the target word }\end{array}$ \\
\hline
\end{tabular}




\section{Appendix 6. Regression models predicting the responses in the survey (A6.1) The influence of item factors other than blend type and definition type on the response: the model with random intercepts for item (qID) and participant (uID)}

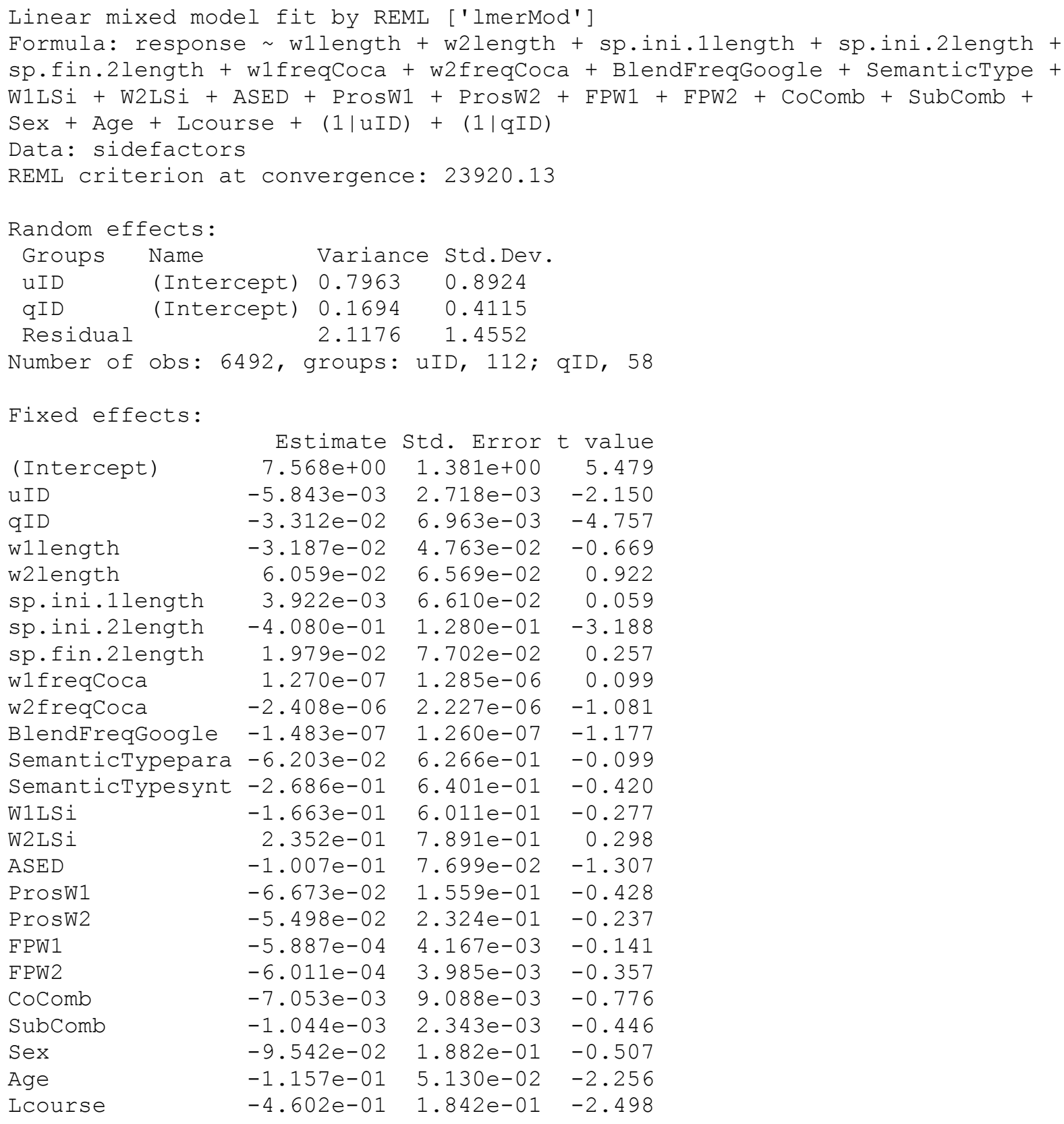


Correlations of fixed effects:

\begin{tabular}{|c|c|c|c|c|}
\hline & (Intercept) & UID & qID & wllength \\
\hline (Intercept) & $1.908105 e+00$ & $-5.712539 e-04$ & $-3.038887 e-03$ & $7.759748 e-04$ \\
\hline uID & $-5.712539 e-04$ & $7.385524 e-06$ & $5.078931 e-10$ & $-4.913008 e-10$ \\
\hline qID & $-3.038887 e-03$ & $5.078931 e-10$ & $4.847991 e-05$ & $-8.640239 e-05$ \\
\hline w1length & $7.759748 e-04$ & $-4.913008 e-10$ & $-8.640239 e-05$ & $2.268152 e-03$ \\
\hline w2length & $-2.523748 e-02$ & $-4.462914 e-09$ & $-4.474100 e-05$ & $2.239374 e-04$ \\
\hline sp.ini.1length & $7.485970 e-03$ & $1.585378 e-09$ & $8.889503 e-06$ & $-1.319201 e-03$ \\
\hline sp.ini.2length & $-3.900861 e-02$ & $6.975270 e-09$ & $3.620299 e-04$ & $-1.067163 e-03$ \\
\hline sp.fin.2length & $3.483738 e-02$ & $2.529345 e-09$ & $-2.693199 e-05$ & $-3.563117 e-04$ \\
\hline wlfreqCoca & $6.669949 e-08$ & $-3.838532 e-14$ & $4.842607 e-11$ & $1.355406 e-08$ \\
\hline w2 freqCoca & $-1.390231 e-06$ & $-2.613553 e-14$ & $3.701432 e-09$ & $-2.741359 e-10$ \\
\hline BlendFreqGoogle & $-7.655831 e-08$ & $5.484154 e-16$ & $2.637012 e-10$ & $-1.851329 e-09$ \\
\hline SemanticTypepara & $-4.581160 e-01$ & $1.349201 e-07$ & $4.547521 e-04$ & $-5.276441 e-03$ \\
\hline SemanticTypesynt & $-5.301546 e-01$ & $1.364968 e-07$ & $8.799318 e-04$ & $-4.479607 e-03$ \\
\hline W1LSi & $-3.440593 e-01$ & $-2.286295 e-09$ & $5.759616 e-04$ & $1.636904 e-02$ \\
\hline W2LSi & $-6.947625 e-01$ & $-1.825766 e-08$ & $9.920742 e-04$ & $-1.193135 e-02$ \\
\hline ASED & $-2.549003 e-02$ & $2.960050 e-09$ & $1.114553 e-04$ & $-1.362962 e-03$ \\
\hline ProsW1 & $-6.291661 e-02$ & $1.089606 e-08$ & $1.285031 e-04$ & $-2.283968 e-04$ \\
\hline Prosw2 & $-1.729185 e-02$ & $-2.450191 e-09$ & $-6.893517 e-04$ & $1.264834 e-03$ \\
\hline FPW1 & $-2.737221 e-03$ & $-5.820850 e-10$ & $4.133300 e-06$ & $2.159675 e-05$ \\
\hline FPW2 & $7.965970 e-03$ & $1.564378 e-09$ & $6.888503 e-06$ & $-1.385201 e-03$ \\
\hline CoComb & $-2.285242 e-03$ & $2.595557 e-10$ & $-5.067510 e-07$ & $9.739330 e-05$ \\
\hline SubComb & $-6.955160 e-04$ & $-6.988639 e-11$ & $-7.511118 e-07$ & $-1.355224 e-05$ \\
\hline Sex & $-6.798516 e-02$ & $9.528007 e-05$ & $2.548714 e-08$ & $-6.874002 e-09$ \\
\hline Age & $-1.080562 e-02$ & $4.867755 e-06$ & $1.149077 e-09$ & $4.661446 e-09$ \\
\hline \multirow[t]{2}{*}{ Lcourse } & $-6.040932 e-02$ & $-1.315541 e-05$ & $-2.378685 e-08$ & 2.191 \\
\hline & Sex & Age & Lcourse & \\
\hline (Intercept) & $-6.798516 e-02$ & $-1.080562 e-02$ & $-6.040932 e-02$ & \\
\hline UID & $9.528007 e-05$ & $4.867755 e-06$ & $-1.315541 e-05$ & \\
\hline qID & $2.548714 e-08$ & $1.149077 e-09$ & $-2.378685 e-08$ & \\
\hline wllength & $-6.874002 e-09$ & $4.661446 e-09$ & $2.191727 e-08$ & \\
\hline w2length & $7.432663 e-08$ & $8.675030 e-08$ & $1.906901 e-07$ & \\
\hline sp.ini.1length & $-2.660688 e-07$ & $-1.086314 e-07$ & $-5.301374 e-08$ & \\
\hline sp.ini.2length & $-5.346259 e-08$ & $-1.152263 e-07$ & $-3.018902 e-07$ & \\
\hline sp.fin.2length & $1.866973 e-07$ & $2.512846 e-08$ & $-1.221327 e-07$ & \\
\hline w1freqCoca & $-2.999712 e-12$ & $-4.353748 e-13$ & $1.863709 e-12$ & \\
\hline w2 freqCoca & $-6.605907 e-13$ & $1.522192 \mathrm{e}-13$ & $1.184044 \mathrm{e}-12$ & \\
\hline BlendFreqGoogle & $1.410403 e-14$ & $-3.115361 e-15$ & $-2.486037 e-14$ & \\
\hline SemanticTypepara & $-3.367675 e-06$ & $-2.986445 e-06$ & $-5.695970 e-06$ & \\
\hline SemanticTypesynt & $-3.271452 e-06$ & $-2.977326 e-06$ & $-5.770866 e-06$ & \\
\hline W1LSi & $1.463689 e-06$ & $5.073097 e-07$ & $1.009359 e-08$ & \\
\hline W2LSi & $-1.193209 e-06$ & $-1.312438 e-07$ & $8.721056 e-07$ & \\
\hline ASED & $1.721092 e-07$ & $1.434891 e-08$ & $-1.400801 e-07$ & \\
\hline ProsW1 & $3.079093 e-07$ & $-5.290733 e-08$ & $-4.956325 e-07$ & \\
\hline ProsW2 & $-1.579676 e-06$ & $-4.785122 e-07$ & $2.042589 e-07$ & \\
\hline FPW1 & $1.527449 e-08$ & $1.312640 e-08$ & $2.452829 e-08$ & \\
\hline CoComb & $2.593550 e-08$ & $4.779000 e-09$ & $-1.294939 e-08$ & \\
\hline SubComb & $3.601054 e-09$ & $2.149749 e-09$ & $2.836339 e-09$ & \\
\hline Sex & $3.540602 e-02$ & $3.805300 e-04$ & $1.157180 e-03$ & \\
\hline Age & $3.805300 e-04$ & $2.631358 e-03$ & $7.899854 e-04$ & \\
\hline Lcourse & $1.157180 e-03$ & $7.899854 e-04$ & $3.392440 e-02$ & \\
\hline
\end{tabular}




\section{(A6.2) The model with random slope for linguistic education:}

Linear mixed model fit by REML ['lmerMod']

Formula: response $\sim$ wllength + w2length + sp.ini.1length + sp.ini.2length + sp.fin.2length + w1freqCoca + w2freqCoca + BlendFreqGoogle + SemanticType + W1LSi + W2LSi + ASED + ProsW1 + ProsW2 + FPW1 + FPW2 + CoComb + SubComb + Sex + Age + Lcourse + (1 | uID $)+(1+$ Age | qID $)+(1+$ Lcourse | qID $)$ Data: sidefactors

REML criterion at convergence: 23915.08

Random effects:

$\begin{array}{lllll}\text { Groups } & \text { Name } & \text { Variance } & \text { Std.Dev. Corr } \\ \text { uID } & \text { (Intercept) } & 0.79652 & 0.8925 & \\ \text { qID } & \text { (Intercept) } & 0.31039 & 0.5571 & \\ & \text { Lcourse } & 0.04095 & 0.2024 & -0.68 \\ \text { Residual } & & 2.10844 & 1.4520 & \end{array}$

Number of obs: 6492, groups: uID, 112; qID, 58

Fixed effects:

$\begin{array}{lrrr} & \text { Estimate } & \text { Std. Error } & \text { value } \\ \text { (Intercept) } & 7.588 e+00 & 1.381 e+00 & 5.494 \\ \text { uID } & -5.843 e-03 & 2.718 e-03 & -2.150 \\ \text { qID } & -3.388 e-02 & 6.956 e-03 & -4.871 \\ \text { w1length } & -3.035 e-02 & 4.758 e-02 & -0.638 \\ \text { w2length } & 6.494 e-02 & 6.562 e-02 & 0.990 \\ \text { sp.ini.1length } & 2.389 e-04 & 6.604 e-02 & 0.004 \\ \text { sp.ini.2length } & -4.163 e-01 & 1.278 e-01 & -3.256 \\ \text { sp.fin.2length } & 1.888 e-02 & 7.695 e-02 & 0.245 \\ \text { w1freqCoca } & 1.393 e-07 & 1.284 e-06 & 0.109 \\ \text { w2freqCoca } & -2.549 e-06 & 2.225 e-06 & -1.145 \\ \text { BlendFreqGoogle } & -1.543 e-07 & 1.259 e-07 & -1.225 \\ \text { SemanticTypepara } & -5.942 e-02 & 6.261 e-01 & -0.095 \\ \text { SemanticTypesynt } & -2.644 e-01 & 6.395 e-01 & -0.413 \\ \text { W1LSi } & -1.854 e-01 & 6.005 e-01 & -0.309 \\ \text { W2LSi } & 2.466 e-01 & 7.884 e-01 & 0.313 \\ \text { ASED } & -1.039 e-01 & 7.692 e-02 & -1.351 \\ \text { ProsW1 } & -5.524 e-02 & 1.558 e-01 & -0.355 \\ \text { Prosw2 } & -4.515 e-02 & 2.322 e-01 & -0.194 \\ \text { FPW1 } & -4.093 e-04 & 4.163 e-03 & -0.098 \\ \text { FPW2 } & -5.018 e-04 & 4.488 e-03 & -0.184 \\ \text { CoComb } & -7.578 e-03 & 9.080 e-03 & -0.835 \\ \text { SubComb } & -1.103 e-03 & 2.340 e-03 & -0.471 \\ \text { Sex } & -9.542 e-02 & 1.882 e-01 & -0.507 \\ \text { Age } & -1.157 e-01 & 5.130 e-02 & -2.256 \\ \text { LCourse } & -4.601 e-01 & 1.861 e-01 & -2.473 \\ & & & \end{array}$

Correlations of fixed effects:

$\begin{array}{lllll} & \text { (Intercept) } & \text { uID } & \text { qID } & \text { w1length } \\ \text { (Intercept) } & 1.90748200 & -0.00057129 & -0.00303307 & 0.00077454 \\ \text { uID } & -0.00057129 & 0.00000739 & 0.00000000 & 0.00000000 \\ \text { qID } & -0.00303307 & 0.00000000 & 0.00004839 & -0.00008624 \\ \text { w1length } & 0.00077454 & 0.00000000 & -0.00008624 & 0.00226380 \\ \text { w2length } & -0.02518915 & 0.00000000 & -0.00004466 & 0.00022351 \\ \text { sp.ini.1length } & 0.00747182 & 0.00000000 & 0.00000887 & -0.00131667 \\ \text { sp.ini.2length } & -0.03893366 & 0.00000001 & 0.00036134 & -0.00106512 \\ \text { sp.fin.2length } & 0.03477048 & 0.00000000 & -0.00002688 & -0.00035562 \\ \text { w1freqCoca } & 0.00000007 & 0.00000000 & 0.00000000 & 0.00000001 \\ \text { w2freqCoca } & -0.00000139 & 0.00000000 & 0.00000000 & 0.00000000 \\ \text { BlendFreqGoogle } & -0.00000008 & 0.00000000 & 0.00000000 & 0.00000000 \\ \text { SemanticTypepara } & -0.45726850 & 0.00000015 & 0.00045388 & -0.00526631\end{array}$




SemanticTypesynt
W1LSi
W2LSi
ASED
ProsW1
ProsW2
FPW1
FPW2
CoComb
SubComb
Sex
Age
Lcourse

\section{(Intercept)}

UID

qID

wllength

w2length

sp.ini.1length

sp.ini.2length

sp.fin.2length

w1 freqCoca

w2 freqCoca

BlendFreqGoogle

SemanticTypepara

SemanticTypesynt

W1LSi

W2LSi

ASED

ProsW1

Prosw2

EPW1

FPW2

CoComb

SubComb

Sex

Age

Lcourse

\section{(Intercept)}

UID

qID

w1length

w2length

sp.ini.1length

sp.ini.2length

sp.fin.2length

w1 freqCoca

w2 freqCoca

BlendFreqGoogle

SemanticTypepara

SemanticTypesynt
$-0.52916910 \quad 0.00000015$

$-0.34339980 \quad 0.00000000$

$-0.69342940-0.00000002$

$-0.025441240 .00000000$

$-0.062796010 .00000001$

$-0.01725770 \quad 0.00000000$

$-0.002731960 .00000000$

$-0.009487760 .00000000$

$-0.002280870 .00000000$

$-0.000694180 .00000000$

$\begin{array}{lll}-0.06798830 & 0.00009528\end{array}$

$-0.010805920 .00000487$

0.00087824

$-0.00447102$

0.00057487

0.00099016

0.00011124

0.01633760

$0.00012826-0.00022797$

$-0.00068804 \quad 0.00126241$

$0.00000413 \quad 0.00002156$

$0.00007468 \quad 0.00084960$

$-0.000000510 .00009721$

$-0.00000075-0.00001353$

$0.00000003-0.00000001$

$0.00000000 \quad 0.00000001$

$-0.06172822-0.00001316-0.000000030 .00000002$

w2length sp.ini.1lth sp.ini.2lth sp.fin.2lth $-0.025189150 .00747182$

$0.00000000 \quad 0.00000000$

$-0.000044660 .00000887$

$-0.038933660 .03477048$

0.000000010 .00000000

0.00022351

$-0.00131667$

$0.00036134-0.00002688$

0.00430664

0.00017198

0.00017198

$-0.00106512-0.00035562$

.00436146

$-0.00363779$

0.00208775

$-0.00312794$

0.00208775

0.01634508

0363779

0.00000000

0.00033687

0.00503328

0.00000001

.00033687

0.00000002

0.00000000

0.00000000

0.00000002

0.00000007

503328

0.00592075

0.00000000

$-0.00355880$

0.00000000

0.00000001

$-0.00000001$

$-0.00126580$

$-0.00359640$

0.00129462

0.00000000

0.00103895

0.00628874

$-0.00228050$

0.02862528

$-0.01577518$

0.01009258

$-0.00414148$

$-0.00775646$

$-0.00192939-$

$-0.002828160$.

$-0.03597536$

$-0.00188316$

$-0.00069593$

$-0.00059982$

0.00055316

$-0.00140697$

0.00449977

0.00222294

0.00127055

0.00003830

0.00002396

$-0.00054503-0.00129265$

0.00073859

0.000078489

0.00020198

$-0.00001882$

0.00007523

$-0.00002165$

0.00847941

$-0.00017173$

0.00003794

0.00000279

0.00009831

$-0.00000311$

0.00000008

$-0.00000029-0$.

$-0.00002618-0.00003101$

0.00000009

$-0.00000012$

$-0.00000006$

0.00000020

0.00000021

$-0.00000006-$

$-0.000000130 .00000003$

w1 freqCoca

0.00000007

w2 freqCoca

$-0.00000139$

BFreqGoogle STypepara

0.00000000

0.00000000

$-0.00000008$

$-0.45726850$

0.00000000

0.00000000

0.00000000

0.00000015

0.00000001

0.00000000

0.00000000

0.00045388

0.00000000

0.00000000

$-0.00526631$

0.00000000

0.00000000

$-0.00355880$

0.00000001

0.00000002

0.00000000

$-0.00359640$

0.00000000

0.00000002

0.00000001

0.00129462

0.00000000

0.00000000

$-0.00228050$

0.00000000

0.00000000

0.00000002

0.00000000

0.00000000

0.00000072

0.00000000

0.00000000

0.00000001

0.00000002

0.00000000

0.00000001

0.39195350

$-0.000000030 .00000085$

0.00000001

0.38517530 


\begin{tabular}{|c|c|c|c|c|}
\hline W1LSi & 0.00000002 & 0.00000019 & 0.00000001 & -0.00366008 \\
\hline W2LSi & -0.00000011 & 0.00000025 & 0.00000005 & 0.10281210 \\
\hline ASED & -0.00000003 & -0.00000003 & 0.00000000 & 0.01091092 \\
\hline ProsW1 & -0.00000002 & -0.00000004 & 0.00000000 & 0.01102101 \\
\hline ProsW2 & -0.00000002 & -0.00000002 & 0.00000000 & -0.00210092 \\
\hline FPW1 & 0.00000000 & 0.00000000 & 0.00000000 & 0.00026466 \\
\hline FPW2 & 0.00000000 & 0.00000000 & 0.00000000 & 0.00738494 \\
\hline CoComb & -0.00000001 & 0.00000000 & 0.00000000 & -0.00107346 \\
\hline SubComb & 0.00000000 & 0.00000000 & 0.00000000 & -0.00020605 \\
\hline Sex & 0.00000000 & 0.00000000 & 0.00000000 & -0.00000368 \\
\hline Age & 0.00000000 & 0.00000000 & 0.00000000 & -0.00000326 \\
\hline \multirow[t]{2}{*}{ Lcourse } & 0.00000000 & 0.00000000 & 0.00000000 & -0.00000622 \\
\hline & STypesynt & W1LSi & W2LSi & ASED \\
\hline (Intercept) & -0.52916910 & -0.34339980 & -0.69342940 & -0.02544124 \\
\hline UID & 0.00000015 & 0.00000000 & -0.00000002 & 0.00000000 \\
\hline qID & 0.00087824 & 0.00057487 & 099016 & 0.00011124 \\
\hline w1length & -0.00447102 & 0.01633760 & -0.01190851 & -0.00136035 \\
\hline w2length & -0.00126580 & 0.00103895 & 0.02862528 & -0.00192939 \\
\hline sp.ini.1length & -0.00268630 & -0.01577518 & -0.00282816 & -0.00127158 \\
\hline sp.ini.2length & 0.00628874 & 0.01009258 & 0.00409949 & -0.00059982 \\
\hline sp.fin.2length & -0.00414148 & -0.00775646 & -0.03597536 & 0.00055316 \\
\hline w1 freqCoca & -0.00000003 & 0.00000002 & -0.00000011 & -0.00000003 \\
\hline w2 freqCoca & 0.000 & 0.00000019 & 0.00000025 & -0.00000003 \\
\hline BlendFreqGoogle & 0.00000001 & 0.00000001 & 0.00000005 & 0.00000000 \\
\hline SemanticTypepara & 0.38517530 & -0.00366008 & 0.10281210 & 0.01091092 \\
\hline SemanticTypesynt & 0.40901910 & 0.02921981 & 0.12053720 & 0.00837878 \\
\hline W1LSi & 0.02921981 & 0.36061280 & 0.00894979 & -0.00309864 \\
\hline W2LSi & 0.12053720 & 0.00894979 & 0.62154070 & 0.01500580 \\
\hline ASED & 0.00837878 & -0.00309864 & 0.01500580 & 0.00591620 \\
\hline ProsW1 & 0.01314718 & 0.02459912 & 0.00953429 & 0.00456071 \\
\hline ProsW2 & -0.01154293 & -0.01863285 & 0.01314317 & 0.00294441 \\
\hline FPW1 & 0.00039336 & 0.00123085 & 0.00092547 & -0.00003916 \\
\hline FPW2 & 0.00031446 & 0.00283934 & 0.00889221 & -0.00098372 \\
\hline CoComb & -0.00027750 & 0.00250182 & -0.00000535 & -0.00004221 \\
\hline SubComb & -0.00023441 & -0.00004896 & 0.00078243 & 0.00005276 \\
\hline Sex & -0.00000357 & 0.00000160 & -0.00000130 & 0.00000019 \\
\hline Age & -0.00000325 & 0.00000055 & -0.00000014 & 0.00000002 \\
\hline \multirow[t]{2}{*}{ Lcourse } & -0.00000630 & 0.00000001 & 0.00000095 & -0.00000015 \\
\hline & ProsW1 & ProsW2 & FPW1 & CoComb \\
\hline (Intercept) & -0.06279601 & -0.01725770 & -0.00273196 & -0.00228087 \\
\hline UID & 0.00000001 & 0.00000000 & 0.00000000 & 0.00000000 \\
\hline qID & 0.00012826 & -0.00068804 & 0.00000413 & -0.00000051 \\
\hline w1length & -0.00022797 & 0.00126241 & 0.00002156 & 0.00009721 \\
\hline w2length & -0.00188316 & -0.00140697 & 0.00003830 & 0.00007523 \\
\hline sp.ini.1length & -0.00069593 & 0.00449977 & 0.00002396 & -0.00002165 \\
\hline sp.ini.2length & 0.00222294 & -0.00054503 & 0.00020198 & 0.00009831 \\
\hline sp.fin.2length & 0.00127055 & -0.00129265 & -0.00001882 & -0.00000311 \\
\hline w1 freqCoca & -0.00000002 & -0.00000002 & 0.00000000 & -0.00000001 \\
\hline w2 freqCoca & -0.00000004 & -0.00000002 & 0.00000000 & 0.00000000 \\
\hline BlendFreqGoogle & 0.00000000 & 0.00000000 & 0.00000000 & 0.00000000 \\
\hline SemanticTypepara & 0.01102101 & -0.00210092 & 0.00026466 & -0.00107346 \\
\hline SemanticTypesynt & 0.01314718 & -0.01154293 & 0.00039336 & -0.00027750 \\
\hline W1LSi & 0.02459912 & -0.01863285 & 0.00123085 & 0.00250182 \\
\hline
\end{tabular}




\begin{tabular}{|c|c|c|c|c|c|c|c|c|}
\hline N2LSi & \multicolumn{2}{|c|}{0.00953429} & \multicolumn{2}{|c|}{0.01314317} & \multicolumn{2}{|c|}{0.00092547} & \multicolumn{2}{|c|}{-0.00000535} \\
\hline ASED & \multicolumn{2}{|c|}{0.00456071} & \multicolumn{2}{|c|}{0.00294441} & \multicolumn{2}{|c|}{-0.00003916} & \multicolumn{2}{|c|}{-0.00004221} \\
\hline ProsW1 & \multicolumn{2}{|c|}{0.02427142} & \multicolumn{2}{|c|}{0.00403952} & \multicolumn{2}{|c|}{0.00007957} & \multicolumn{2}{|c|}{0.00026042} \\
\hline ProsW2 & \multicolumn{2}{|c|}{0.00403952} & \multicolumn{2}{|c|}{0.05391654} & \multicolumn{2}{|c|}{-0.00000599} & \multicolumn{2}{|c|}{-0.00014744} \\
\hline FPW1 & \multicolumn{2}{|c|}{0.00007957} & \multicolumn{2}{|c|}{-0.00000599} & \multicolumn{2}{|c|}{0.00001733} & \multicolumn{2}{|c|}{0.00000683} \\
\hline PW2 & \multicolumn{2}{|c|}{0.00019889} & \multicolumn{2}{|c|}{-0.00000726} & \multicolumn{2}{|c|}{0.00003771} & \multicolumn{2}{|c|}{0.00001879} \\
\hline oComb & \multicolumn{2}{|c|}{0.00026042} & \multicolumn{2}{|c|}{-0.00014744} & 0.0000 & 00683 & 0.0000 & 08244 \\
\hline SubComb & 0.000 & 09099 & 0.0001 & 13736 & -0.000 & 000030 & 0.0000 & 00419 \\
\hline Sex & 0.000 & 00034 & -0.000 & 000172 & 0.0000 & 00002 & 0.0000 & 00003 \\
\hline Age & -0.00 & 000006 & -0.000 & 000052 & 0.0000 & 00001 & 0.0000 & 00001 \\
\hline course & -0.00 & 000054 & 0.0000 & 00022 & 0.0000 & 00003 & -0.0 & 000001 \\
\hline & & SubCc & & Sex & & Age & & \\
\hline (Intercept) & & & 069418 & -0.0 & 798830 & -0.0 & 080592 & -0.06172822 \\
\hline UID & & 0.000 & 00000 & 0.00 & 9528 & 0.00 & & -0.00 \\
\hline qID & & -0.000 & 000075 & 0.0000 & 00003 & 0.0000 & 000 & -0.0 \\
\hline wllength & & -0.000 & 001353 & -0.000 & 000001 & 0.0000 & & 0.0 \\
\hline w2length & & 0.0000 & 03794 & 0.0000 & 00008 & 0.0000 & 00009 & 0.000 \\
\hline sp.ini.1leng & & 0.0 & 00279 & -0 & 000029 & -0.0 & 000012 & -0.000 \\
\hline p.ini.2leng & & -0.0 & 002618 & -0.000 & 000006 & -0.000 & 000013 & -0.0000 \\
\hline sp.fin.2leng & & -0.0 & 003101 & 0.0000 & 00020 & 0.0000 & 00003 & -0.000 \\
\hline w1 freqCoca & & 0.00 & 00000 & 0.0000 & 00000 & 0.0000 & 00000 & 0.0000 \\
\hline w2 freqCoca & & 0.00 & 00000 & 0.0000 & 00000 & 0.0000 & 00000 & 0.000 \\
\hline BlendFreqGoo & & 0.00 & 00000 & 0.0000 & 00000 & 0.0000 & 00000 & 0.000 \\
\hline SemanticType & para & -0.0 & 020605 & -0.000 & 000368 & -0.000 & 000326 & -0.00 \\
\hline SemanticType & synt & -0.0 & 023441 & -0.000 & 000357 & -0.000 & 25 & -0.0 \\
\hline W1LSi & & -0.0 & 004896 & 0.0000 & 00160 & 0.0000 & & 0.00 \\
\hline W2LSi & & 0.0 & 3243 & -0.000 & 000130 & -0.000 & 000014 & 0.00 \\
\hline ASED & & 0.00 & 05276 & 0.0000 & 00019 & 0.0000 & & -0.000 \\
\hline ProsW1 & & 0.0000 & 09099 & 0.0000 & 00034 & -0.000 & 000006 & -0.00000054 \\
\hline Prosw2 & & 0.0001 & 13736 & -0.000 & 000172 & -0.000 & 000052 & 0.00000022 \\
\hline FPW1 & & -0.000 & 000030 & 0.0000 & 00002 & 0.0000 & 00001 & 0.00000003 \\
\hline FPW2 & & -0.000 & 019559 & 0.0000 & 00065 & 0.0000 & 00097 & 0.00000033 \\
\hline CoComb & & 0.00 & 00419 & 0.0000 & 00003 & 0.0000 & 00001 & -0.00000001 \\
\hline SubComb & & 0.00 & 00548 & 0.0000 & 00000 & 0.0000 & 00000 & 0.00000000 \\
\hline Sex & & 0.00 & 00000 & 0.0354 & 407 & 0.0003 & 38055 & 0.00115724 \\
\hline Age & & & 00000 & 0.0003 & & 0.0026 & 63149 & 0.00079002 \\
\hline Lcourse & & 0.0000 & 00000 & 0.0011 & 15724 & 0.0007 & 79002 & 0.03463209 \\
\hline
\end{tabular}




\section{(A6.3) The model with random slopes for age and linguistic education:}

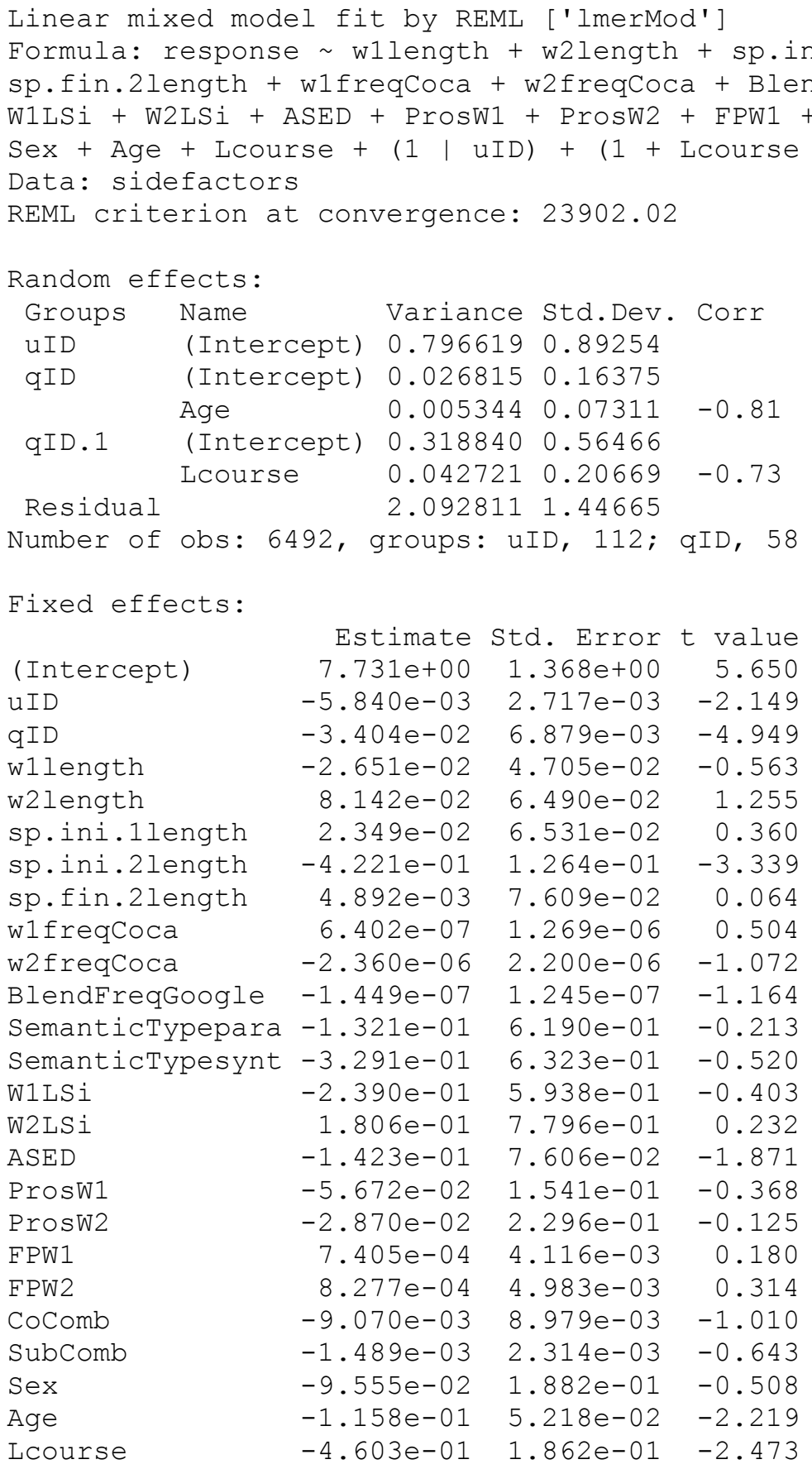

Correlation matrix of fixed effects:

$\begin{array}{lllll} & \text { (Intercept) } & \text { uID } & \text { qID } & \text { w1length } \\ \text { (Intercept) } & 1.87228100 & -0.00057111 & -0.00296619 & 0.00075728 \\ \text { uID } & -0.00057111 & 0.00000738 & 0.00000000 & 0.00000000 \\ \text { qID } & -0.00296619 & 0.00000000 & 0.00004732 & -0.00008433 \\ \text { w1length } & 0.00075728 & 0.00000000 & -0.00008433 & 0.00221377 \\ \text { w2length } & -0.02463190 & 0.00000000 & -0.00004367 & 0.00021858 \\ \text { sp.ini.1length } & 0.00730700 & 0.00000000 & 0.00000866 & -0.00128758 \\ \text { sp.ini.2length } & -0.03807444 & 0.00000000 & 0.00035336 & -0.00104158 \\ \text { sp.fin.2length } & 0.03400099 & 0.00000000 & -0.00002627 & -0.00034780 \\ \text { w1freqCoca } & 0.00000007 & 0.00000000 & 0.00000000 & 0.00000001 \\ \text { w2freqCoca } & -0.00000136 & 0.00000000 & 0.00000000 & 0.00000000 \\ \text { BlendFreqGoogle } & -0.00000007 & 0.00000000 & 0.00000000 & 0.00000000 \\ \text { SemanticTypepara } & -0.44702600 & 0.00000007 & 0.00044383 & -0.00514995\end{array}$




SemanticTypesynt
W1LSi
W2LSi
ASED
ProsW1
ProsW2
FPW1
CoComb
SubComb
Sex
Age
Lcourse

(Intercept)
uID
qID
w1length
w2length
sp.ini.1length
sp.ini.2length
sp.fin.2length
w1freqCoca
w2freqCoca
BlendFreqGoogle
SemanticTypepara
SemanticTypesynt
W1LSi
W2LSi
ASED
ProsW1
ProsW2
FPW1
CoComb
SubComb
Sex
Age
Lcourse

(Intercept)
uID
qID
w1length
w2length
sp.ini.1length
sp.ini.2length
sp.fin.2length
w1 freqCoca
w2freqCoca
BlendFreqGoogle
SemanticTypepara
SemanticTypesynt
W1LSi
W2LSi
ASED
ProsW1
ProsW2
FPW1
CoComb
SubComb
Sex
Age

$-0.51733770 \quad 0.00000008$ $-0.33581630 \quad 0.00000001$ $-0.67809740-0.00000002$ $-0.024879860 .00000000$ $-0.061410990 .00000001$ $-0.01687163-0.00000001$ $-0.002671630 .00000000$ $-0.002230590 .00000000$ $-0.000678850 .00000000$ $-0.067976370 .00009527$ $-0.010973390 .00000487$ $-0.06187889-0.00001315$

w2length sp.ini.11 $-0.02463190 \quad 0.00730700$ $0.00000000 \quad 0.00000000$ $-0.00004367 \quad 0.00000866$ 0.00021858

0.00421141

0.00016825

$-0.00128758$

$-0.00305874$

0.00016825

0.00426515

$-0.00355739$

0.00000000

0.00000002

0.00000000

0.00204151

0.00032929

0.00000000

0.00000002

0.00000000

$-0.00348017$

$-0.00123787$

0.00101571

0.02799248

$-0.00351683$

$-0.002626850$.

0.00085883

0.00056228

0.00096814

0.00010880

0.00012546

$-0.00067297$

0.00000404

$-0.00000049$

$-0.00000073$

0.00000005

0.00000001

$-0.00000003$

$-0.00437219$

0.01597662

$-0.01164504$

$-0.00133029$

$-0.00022292$

0.00123458

0.00002107

0.00009506

$-0.00001323$

$-0.00000002$

0.00000000

0.00000002

sp.ini.21 sp.fin.21

$-0.038074440 .03400099$

$0.00000000 \quad 0.00000000$

$0.00035336-0.00002627$

$-0.00104158-0.00034780$

$-0.00305874-0.00355739$

$\begin{array}{lll}0.00204151 & 0.00032929\end{array}$

$0.01598383 \quad 0.00492211$

$0.00492211 \quad 0.00579003$

$0.00000001 \quad 0.00000000$

$0.00000007-0.00000001$

$0.00000001 \quad 0.00000000$

$0.00126598-0.00223023$

$-0.00758433$

$-0.03518083$

$\begin{array}{lllll}-0.00188677 & -0.00124359 & -0.00058656 & 0.00054107\end{array}$

$\begin{array}{lllll}-0.00184148 & -0.00068087 & 0.00217376 & 0.00124274\end{array}$

$\begin{array}{lllll}-0.00137567 & 0.00440100 & -0.00053350 & -0.00126493\end{array}$

0.00003744

0.00007357

0.00003710

0.00000001

0.00000004

0.00000012

0.00002343

$-0.00002118$

$0.00019754-0.00001840$

$0.00000273-0.00002559-0.00003032$

$\begin{array}{llll}-0.00000037 & 0.00000007 & 0.00000034\end{array}$

$-0.00000011-0.000000040 .00000007$

$0.00000005-0.00000020-0.00000015$

w1 frCoca

0.00000007

0.00000000

0.00000000

w2 frCoca

BFrGoogle

SemTypepara

$-0.00000136-0.00000007-0.44702600$

0.00000000

0.00000000

0.00000000

0.00000007

0.00000001

0.00000000

0.00000000

0.00000000

0.00000000

0.00044383

0.00000002

0.00000000

0.00000002

0.00000000

$-0.00514995$

0.00000001

0.00000000

0.00000000

0.00000000

0.00000007

0.00000000

$-0.00348017$

0.00000007

0.00000001

$-0.00351683$

0.00000000

0.00000000

0.00000000

0.00000000

0.00000000

0.00000000

0.00000000

0.00000000

0.00000071

0.00000002

0.00000001

0.00000001

0.00000001

0.00000002

0.00000005

.000000100 .00000024

$-0.00000003$

0.00000000

$-0.00000002$

.00000003

0.00000000

$-0.00000001$

$-0.00000002$

0.00000000

0.00000000

0.00000000

$-0.00000001$

0.00000000

0.00000000

0.00000000

0.00000000

0.00000000

0.00000000

0.00000000

0.00000000

0.00000000

0.00000000

0.00000000

0.00126598

$-0.00223023$

0.00000002

0.00000071

0.00000001

0.38314520

0.37651680

$-0.00357989$

0.10054140

0.01066975

0.01077728

$-0.00205374$

0.00025882

$-0.00104976$

$-0.00020150$

$-0.00000210$

$-0.00000171$ 
Lcourse

(Intercept)

UID

qID

wllength

w2length

sp.ini.1length

sp.ini.2length

sp.fin.2length

w1freqCoca

w2 freqCoca

BlendFreqGoogle

SemanticTypepara

SemanticTypesynt

W1LSi 0.02857386

W2LSi 0.11787340

ASED 0.00819356

ProsW1

ProsW2

FPW1

CoComb

SubComb

Sex

Age

Lcourse

(Intercept)

UID

qID

wllength

w2length

sp.ini.1length

sp.ini.2length

sp.fin.2length

w1 freqCoca

w2 freqCoca

BlendFreqGoogle

SemanticTypepara

SemanticTypesynt

W1LSi

W2LSi

ASED

Prosh1

Prosh2

FPW1

CoComb

SubComb

Sex

Age

Lcourse

(Intercept)

UID

qID

wllength

w2length

sp.ini.1length

sp.ini.2length

sp.fin.2length
$0.00000000 \quad 0.00000000 \quad 0.00000000 \quad-0.00000309$

SemTypesynt W1LSi W2LSi ASED

$\begin{array}{llll}-0.51733770 & -0.33581630 & -0.67809740 & -0.02487986\end{array}$

$\begin{array}{llll}0.00000008 & 0.00000001 & -0.00000002 & 0.00000000\end{array}$

$\begin{array}{lllll}0.00085883 & 0.00056228 & 0.00096814 & 0.00010880\end{array}$

$\begin{array}{lllll}-0.00437219 & 0.01597662 & -0.01164504 & -0.00133029\end{array}$

$\begin{array}{lllll}-0.00123787 & 0.00101571 & 0.02799248 & -0.00188677\end{array}$

$-0.00262685-0.01542714-0.00276451-0.00124359$

$\begin{array}{lllll}0.00614977 & 0.00987015 & 0.00400880 & -0.00058656\end{array}$

$-0.00404997-0.00758433-0.035180830 .00054107$

$\begin{array}{llll}-0.00000003 & 0.00000002 & -0.00000010 & -0.00000003\end{array}$

$\begin{array}{lllll}0.00000083 & 0.00000019 & 0.00000024 & -0.00000003\end{array}$

$\begin{array}{lllll}0.00000001 & 0.00000001 & 0.00000005 & 0.00000000\end{array}$

$\begin{array}{lllll}0.37651680 & -0.00357989 & 0.10054140 & 0.01066975\end{array}$

$\begin{array}{lllll}0.39983350 & 0.02857386 & 0.11787340 & 0.00819356\end{array}$

$0.35264910 \quad 0.00874648 \quad-0.00302949$

$0.00874648 \quad 0.60780660 \quad 0.01467321$

$\begin{array}{lll}-0.00302949 & 0.01467321 & 0.00578558\end{array}$

$0.01285654 \quad 0.02405745 \quad 0.00932176$

$\begin{array}{lllll}-0.01128753 & -0.01822541 & 0.01285850 & 0.00287856\end{array}$

$\begin{array}{lllll}0.00038468 & 0.00120365 & 0.00090509 & -0.00003829\end{array}$

$\begin{array}{lllll}-0.00027138 & 0.00244664 & -0.00000533 & -0.00004126\end{array}$

$\begin{array}{lllll}-0.00022923 & -0.00004788 & 0.00076514 & 0.00005160\end{array}$

$\begin{array}{lllll}-0.00000192 & 0.00000216 & -0.00000221 & 0.00000033\end{array}$

$\begin{array}{llll}-0.00000168 & 0.00000060 & -0.00000043 & 0.00000006\end{array}$

$-0.00000317-0.000000460 .00000101-0.00000016$

ProsW1 $\quad$ ProsW2 $\quad$ FPW1 $\quad$ CoComb

$-0.06141099-0.01687163-0.00267163-0.00223059$

$0.00000001-0.000000010 .00000000 \quad 0.00000000$

$\begin{array}{lllll}0.00012546 & -0.00067297 & 0.00000404 & -0.00000049\end{array}$

$\begin{array}{lllll}-0.00022292 & 0.00123458 & 0.00002107 & 0.00009506\end{array}$

$\begin{array}{lllll}-0.00184148 & -0.00137567 & 0.00003744 & 0.00007357\end{array}$

$\begin{array}{lllll}-0.00068087 & 0.00440100 & 0.00002343 & -0.00002118\end{array}$

$\begin{array}{lllll}0.00217376 & -0.00053350 & 0.00019754 & 0.00009615\end{array}$

$0.00124274-0.00126493-0.00001840-0.00000302$

$\begin{array}{llll}-0.00000002 & -0.00000001 & 0.00000000 & -0.00000001\end{array}$

$\begin{array}{lllll}-0.00000003 & -0.00000002 & 0.00000000 & 0.00000000\end{array}$

$\begin{array}{lllll}0.00000000 & 0.00000000 & 0.00000000 & 0.00000000\end{array}$

$\begin{array}{lllll}0.01077728 & -0.00205374 & 0.00025882 & -0.00104976\end{array}$

$\begin{array}{lllll}0.01285654 & -0.01128753 & 0.00038468 & -0.00027138\end{array}$

$\begin{array}{llll}0.02405745 & -0.01822541 & 0.00120365 & 0.00244664\end{array}$

$\begin{array}{llll}0.00932176 & 0.01285850 & 0.00090509 & -0.00000533\end{array}$

$0.00446014 \quad 0.00287856-0.00003829-0.00004126$

$\begin{array}{llll}0.02373555 & 0.00394830 & 0.00007783 & 0.00025470\end{array}$

$\begin{array}{lllll}0.00394830 & 0.05273040 & -0.00000586 & -0.00014428\end{array}$

$\begin{array}{lllll}0.00007783 & -0.00000586 & 0.00001694 & 0.00000668\end{array}$

$\begin{array}{lllll}0.00025470 & -0.00014428 & 0.00000668 & 0.00008062\end{array}$

$\begin{array}{lllll}0.00008899 & 0.00013432 & -0.00000029 & 0.00000410\end{array}$

$\begin{array}{lllll}0.00000071 & -0.00000244 & 0.00000001 & 0.00000004\end{array}$

$\begin{array}{lllll}0.00000010 & -0.00000063 & 0.00000001 & 0.00000001\end{array}$

$\begin{array}{lllll}-0.00000046 & 0.00000065 & 0.00000001 & -0.00000002\end{array}$

SubComb Sex Age Lcourse

$-0.00067885-0.06797637-0.01097339-0.06187889$

$\begin{array}{lllll}0.00000000 & 0.00009527 & 0.00000487 & -0.00001315\end{array}$

$\begin{array}{lllll}-0.00000073 & 0.00000005 & 0.00000001 & -0.00000003\end{array}$

$-0.00001323-0.00000002 \quad 0.00000000 \quad 0.00000002$

$\begin{array}{llll}0.00003710 & 0.00000001 & 0.00000004 & 0.00000012\end{array}$

$0.00000273-0.00000037-0.000000110 .00000005$

$\begin{array}{lllll}-0.00002559 & 0.00000007 & -0.00000004 & -0.00000020\end{array}$

$\begin{array}{lllll}-0.00003032 & 0.00000034 & 0.00000007 & -0.00000015\end{array}$ 


$\begin{array}{lllll}\text { w1freqCoca } & 0.00000000 & 0.00000000 & 0.00000000 & 0.00000000 \\ \text { w2freqCoca } & 0.00000000 & 0.00000000 & 0.00000000 & 0.00000000 \\ \text { BlendFreqGoogle } & 0.00000000 & 0.00000000 & 0.00000000 & 0.00000000 \\ \text { SemanticTypepara } & -0.00020150 & -0.00000210 & -0.00000171 & -0.00000309 \\ \text { SemanticTypesynt } & -0.00022923 & -0.00000192 & -0.00000168 & -0.00000317 \\ \text { W1LSi } & -0.00004788 & 0.00000216 & 0.00000060 & -0.00000046 \\ \text { W2LSi } & 0.00076514 & -0.00000221 & -0.00000043 & 0.00000101 \\ \text { ASED } & 0.00005160 & 0.00000033 & 0.00000006 & -0.00000016 \\ \text { ProsW1 } & 0.00008899 & 0.00000071 & 0.00000010 & -0.00000046 \\ \text { ProsW2 } & 0.00013432 & -0.00000244 & -0.00000063 & 0.00000065 \\ \text { FPW1 } & -0.00000029 & 0.00000001 & 0.00000001 & 0.00000001 \\ \text { CoComb } & 0.00000410 & 0.00000004 & 0.00000001 & -0.00000002 \\ \text { SubComb } & 0.00000536 & 0.00000000 & 0.00000000 & 0.00000000 \\ \text { Sex } & 0.00000000 & 0.03540045 & 0.00038047 & 0.00115700 \\ \text { Age } & 0.00000000 & 0.00038047 & 0.00272309 & 0.00078986 \\ \text { Lcourse } & 0.00000000 & 0.00115700 & 0.00078986 & 0.03465563\end{array}$

\section{(A6.4) The influence of simple effects of blend type and definition type on the response in the main survey:}

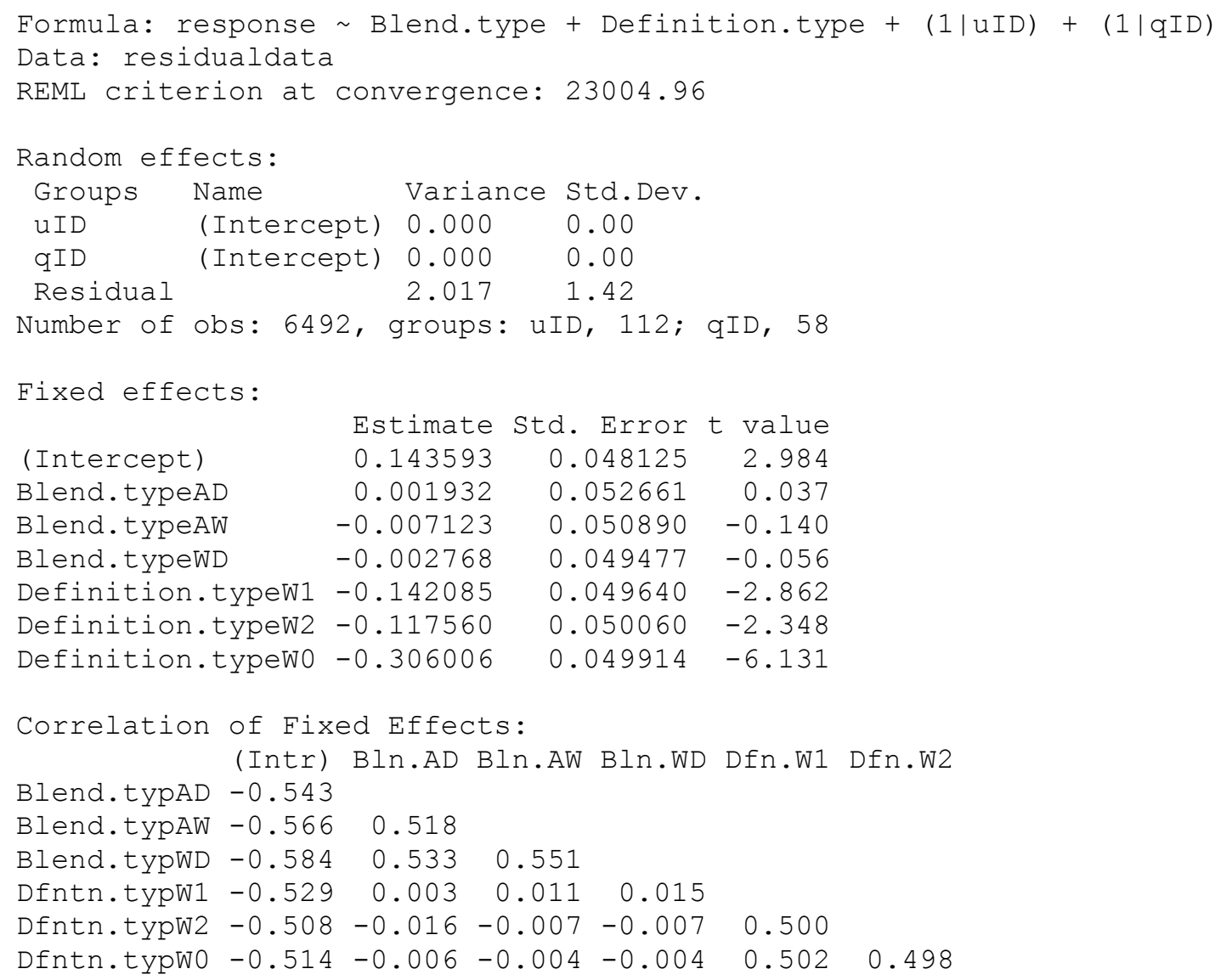


(A6.5) The influence of blend type and definition type on the response in the main survey, accounting for the interaction of the two factors:

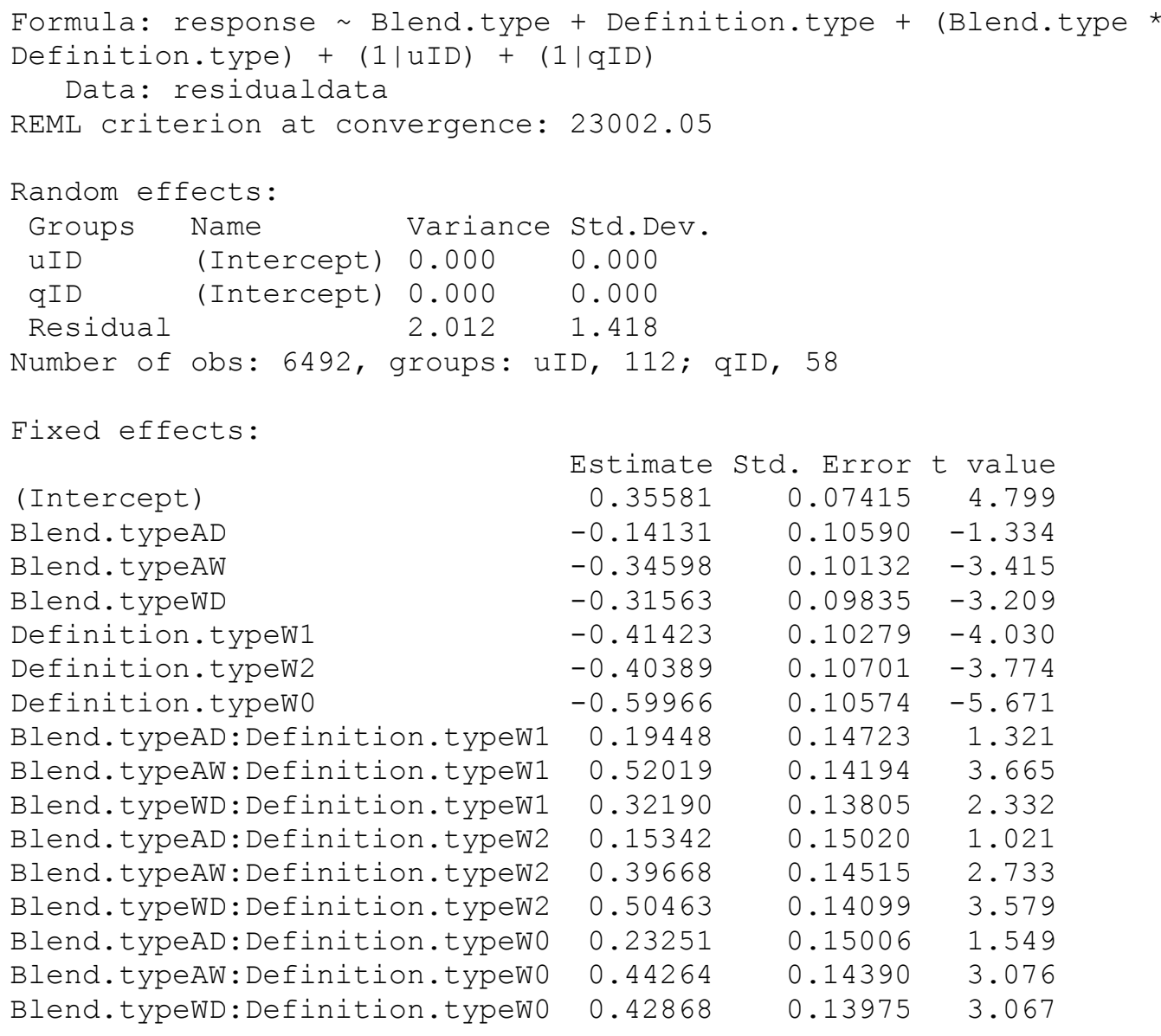

$\begin{array}{rrr}\text { Estimate } & \text { Std. Error } & \text { t value } \\ 0.35581 & 0.07415 & 4.799 \\ -0.14131 & 0.10590 & -1.334 \\ -0.34598 & 0.10132 & -3.415 \\ -0.31563 & 0.09835 & -3.209 \\ -0.41423 & 0.10279 & -4.030 \\ -0.40389 & 0.10701 & -3.774 \\ -0.59966 & 0.10574 & -5.671 \\ 0.19448 & 0.14723 & 1.321 \\ 0.52019 & 0.14194 & 3.665 \\ 0.32190 & 0.13805 & 2.332 \\ 0.15342 & 0.15020 & 1.021 \\ 0.39668 & 0.14515 & 2.733 \\ 0.50463 & 0.14099 & 3.579 \\ 0.23251 & 0.15006 & 1.549 \\ 0.44264 & 0.14390 & 3.076 \\ 0.42868 & 0.13975 & 3.067\end{array}$

(A6.6) The influence of item factors other than blend type and definition type on the response, based on the data from the main and the additional survey:

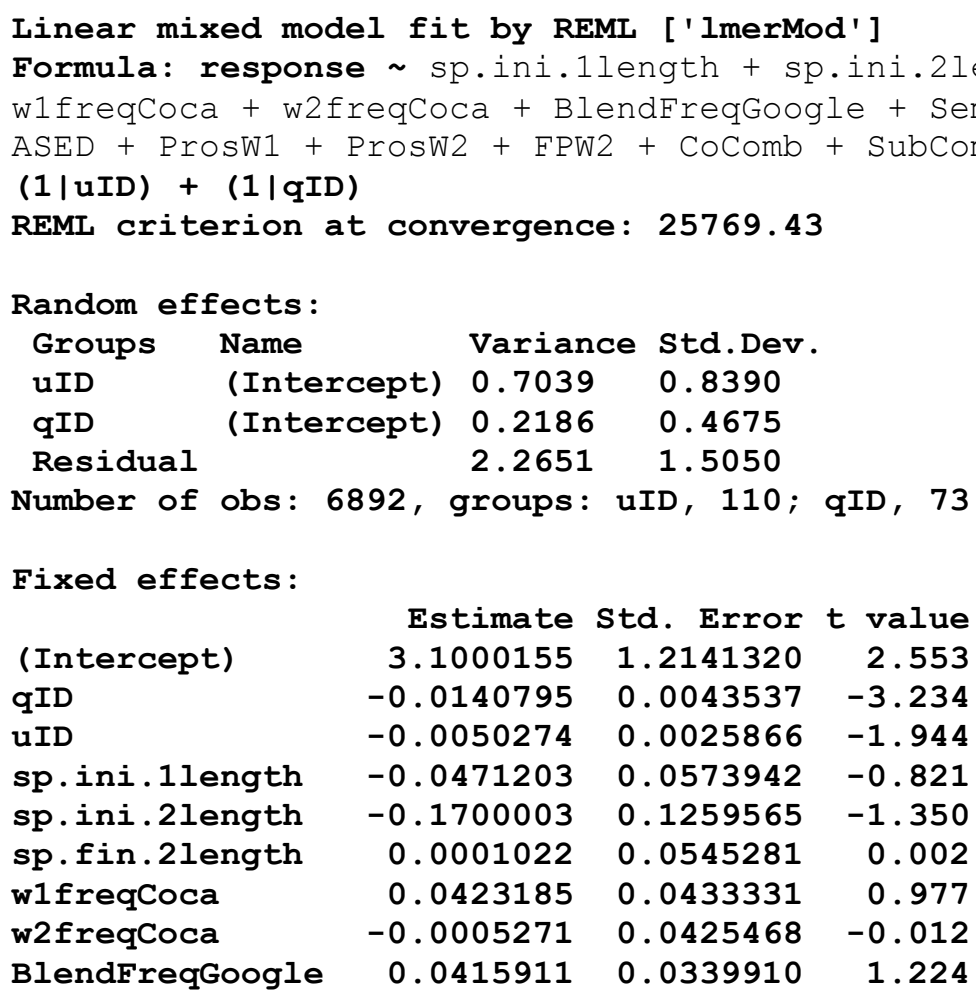




\begin{tabular}{|c|c|c|c|c|c|c|c|c|c|c|}
\hline \multicolumn{2}{|c|}{ SemanticTypesynt } & \multicolumn{2}{|c|}{-0.0361491} & \multicolumn{2}{|c|}{0.1644909} & -0.220 & & & & \\
\hline \multicolumn{2}{|c|}{ W1LSi } & \multicolumn{2}{|c|}{0.8891790} & \multicolumn{2}{|c|}{0.4254454} & 2.090 & & & & \\
\hline \multicolumn{2}{|l|}{ W2LSi } & \multicolumn{2}{|c|}{1.0328152} & \multicolumn{2}{|c|}{0.6125316} & 1.686 & & & & \\
\hline \multicolumn{2}{|l|}{ ASED } & \multicolumn{2}{|c|}{-0.0183969} & \multicolumn{2}{|c|}{0.0623499} & -0.295 & & & & \\
\hline \multicolumn{2}{|l|}{ Prosw1 } & \multicolumn{2}{|c|}{0.0473919} & \multicolumn{2}{|c|}{0.1657792} & 0.286 & & & & \\
\hline \multicolumn{2}{|l|}{ Prosw2 } & \multicolumn{2}{|c|}{-0.0268722} & \multicolumn{2}{|c|}{0.2206264} & -0.122 & & & & \\
\hline FPW2 & & 0.000 & 1582 & 0.00201 & 181 & .078 & & & & \\
\hline CoComb & & -0.005 & 3420 & 0.00788 & 315 & .678 & & & & \\
\hline SubComb & & -0.000 & 2937 & 0.00254 & 484 & .115 & & & & \\
\hline w21.resid & & 0.028 & 1426 & 0.06723 & 356 & .419 & & & & \\
\hline FPW1.resid & & 0.003 & 6003 & 0.00430 & 007 & .837 & & & & \\
\hline Correlatior & $\mathrm{n}$ of $\mathrm{Fj}$ & ixed Ef: & fects: & & & & & & & \\
\hline (Intr) qID & uID & sp.n. & 1 sp.n. & $.2 \mathrm{sp} . \mathrm{f}$ & $.2 \mathrm{w} 1 \mathrm{frq}$ & qC w2frq & qC BlndF & FG Smntc! & TT W1LSi & W2LSi \\
\hline qID & -0.232 & & & & & & & & & \\
\hline UID & -0.130 & 0.022 & & & & & & & & \\
\hline sp.n.1lngth & -0.247 & -0.014 & 0.001 & & & & & & & \\
\hline sp.n.2lngth & -0.535 & 0.014 & 0.011 & 0.210 & & & & & & \\
\hline sp.fn.2lngt & 0.154 & -0.341 & 0.000 & -0.126 & 0.367 & & & & & \\
\hline w1freqCoca & -0.437 & 0.331 & -0.001 & 0.094 & 0.104 & -0.052 & & & & \\
\hline w2freqCoca & -0.445 & 0.165 & 0.002 & -0.025 & 0.294 & 0.101 & 0.170 & & & \\
\hline BlendFrqGgl & -0.517 & 0.086 & 0.007 & 0.360 & 0.231 & -0.211 & 0.032 & -0.031 & & \\
\hline SmntcTypsyn & 0.033 & -0.052 & 0.008 & 0.054 & -0.012 & -0.034 & -0.246 & 0.214 & -0.075 & \\
\hline W1LSi & -0.366 & -0.026 & 0.008 & -0.144 & 0.085 & -0.245 & -0.124 & 0.154 & 0.219 & 0.133 \\
\hline W2LSi & -0.647 & 0.020 & 0.006 & -0.078 & 0.403 & -0.322 & 0.159 & 0.090 & 0.225 & -0.146 \\
\hline ASED & -0.351 & 0.064 & 0.003 & -0.138 & -0.142 & -0.186 & 0.015 & -0.170 & 0.116 & -0.262 \\
\hline Prosw1 & -0.346 & 0.107 & 0.002 & 0.088 & 0.107 & 0.025 & 0.133 & -0.005 & 0.046 & 0.013 \\
\hline ProsW2 & -0.336 & -0.230 & -0.004 & 0.516 & 0.235 & -0.187 & -0.085 & -0.007 & 0.153 & -0.102 \\
\hline FPW2 & -0.334 & 0.032 & 0.004 & -0.039 & 0.301 & 0.009 & 0.183 & 0.224 & -0.104 & -0.042 \\
\hline CoComb & -0.022 & -0.212 & 0.004 & 0.098 & 0.083 & 0.088 & -0.395 & 0.026 & 0.058 & 0.433 \\
\hline SubComb & -0.163 & -0.072 & 0.002 & 0.139 & -0.078 & -0.159 & -0.202 & -0.263 & 0.294 & -0.090 \\
\hline w21.resid & 0.159 & 0.044 & -0.003 & -0.072 & -0.428 & -0.296 & -0.116 & 0.073 & 0.124 & 0.251 \\
\hline FPW1.resid & -0.253 & -0.049 & 0.003 & 0.073 & 0.278 & 0.026 & 0.085 & 0.263 & 0.015 & 0.019 \\
\hline & ASED & ProsW: & 1 ProsI & W2 FPW2 & CoComl & b SubCo & omb & w21. rs & & \\
\hline ProsW1 & 0.253 & 0.132 & 0.309 & 0.088 & 0.107 & 0.025 & & 0.133 & & \\
\hline Prosw2 & -0.111 & 0.371 & 0.308 & 0.516 & 0.235 & -0.187 & & -0.085 & & \\
\hline FPW2 & 0.164 & 0.439 & -0.120 & -0.039 & 0.301 & 0.009 & & 0.183 & & \\
\hline CoComb & 0.391 & -0.088 & -0.086 & 0.098 & 0.083 & 0.088 & & -0.395 & & \\
\hline SubComb & 0.096 & 0.291 & 0.231 & 0.139 & -0.078 & -0.159 & & -0.202 & & \\
\hline w21.resid & 0.055 & -0.110 & -0.298 & -0.072 & -0.428 & -0.296 & & -0.116 & & \\
\hline FPW1.resid & 0.086 & 0.223 & -0.043 & 0.073 & 0.278 & 0.026 & & 0.085 & & \\
\hline
\end{tabular}

(A6.7) The influence of simple effects of blend type and definition type on the response in the main and additional survey:

Linear mixed model fit by maximum likelihood ['lmerMod']

Formula: response Blend.type + Definition.type + (1/uID) + (1|qID) Data: ww.residualdata

$\begin{array}{rrrr}\text { AIC } & \text { BIC } & \text { logLik } & \text { deviance } \\ 25015.67 & 25090.89 & -12496.84 & 24993.67\end{array}$

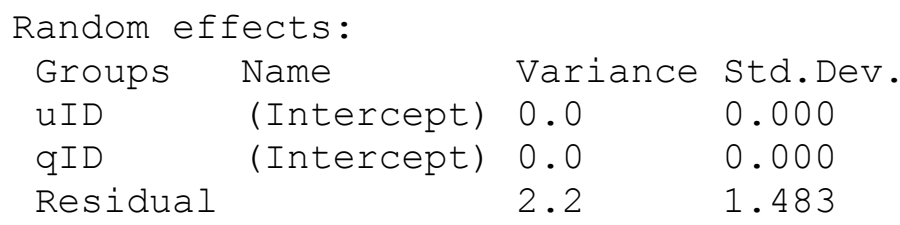

Number of obs: 6892, groups: uID, 110; qID, 73

Fixed effects:

$\begin{array}{lrrr} & \text { Estimate } & \text { Std. Error } t \text { value } \\ \text { (Intercept) } & 0.122380 & 0.050329 & 2.432 \\ \text { Blend.typeAD } & -0.010040 & 0.056266 & -0.178\end{array}$




$\begin{array}{lrrr}\text { Blend.typeAW } & -0.007946 & 0.053582 & -0.148 \\ \text { Blend.typeWD } & -0.011097 & 0.053577 & -0.207 \\ \text { Blend.typeWW } & 0.017108 & 0.069088 & 0.248 \\ \text { Definition.typeW1 } & -0.124132 & 0.050319 & -2.467 \\ \text { Definition.typeW2 } & -0.086284 & 0.050715 & -1.701 \\ \text { Definition.typeW0 } & -0.259037 & 0.050583 & -5.121\end{array}$

Correlation of Fixed Effects:

(Intr) Bln.AD Bln.AW Bln.WD Bln.WW Dfn.W1 Dfn.W2

Blend.typAD -0.555

Blend. typAW -0.587

Blend.typWD $-0.586 \quad 0.525 \quad 0.552$

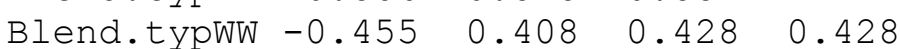

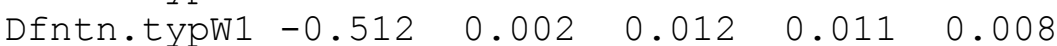

Dentn.typw2 $-0.490 \quad-0.015-0.008-0.011-0.009 \quad 0.500$

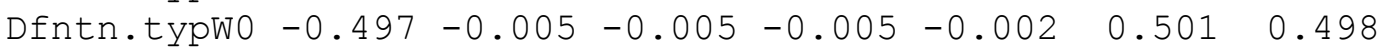

\section{(A6.8) The influence of blend type and definition type on the response in the main and the additional survey, accounting for the interaction of the two factors:}

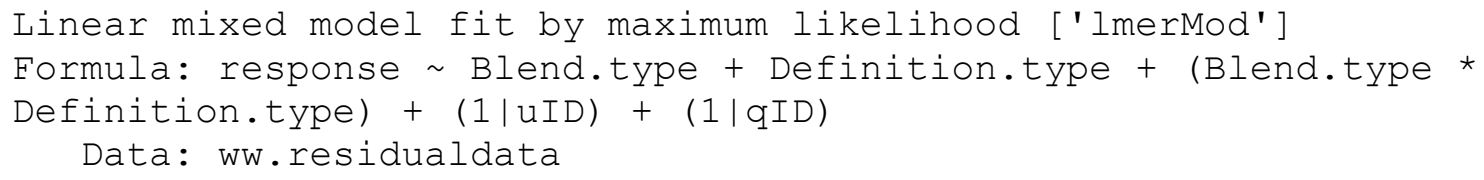

$\begin{array}{rrr}\text { Estimate } & \text { Std. Error } & t \text { value } \\ 0.36195 & 0.07886 & 4.590 \\ -0.14014 & 0.11268 & -1.244 \\ -0.33244 & 0.10624 & -3.129 \\ -0.34681 & 0.10641 & -3.259 \\ -0.47334 & 0.13738 & -3.445 \\ -0.42556 & 0.10953 & -3.886 \\ -0.39733 & 0.11428 & -3.477 \\ -0.61075 & 0.11259 & -5.424 \\ 0.20014 & 0.15675 & 1.277 \\ 0.49381 & 0.14917 & 3.310 \\ 0.36462 & 0.14933 & 2.442 \\ 0.48853 & 0.19334 & 2.527 \\ 0.10867 & 0.16044 & 0.677 \\ 0.37413 & 0.15270 & 2.450 \\ 0.52732 & 0.15250 & 3.458 \\ 0.63790 & 0.19570 & 3.260 \\ 0.22129 & 0.15992 & 1.384 \\ 0.43791 & 0.15096 & 2.901 \\ 0.45588 & 0.15120 & 3.015 \\ 0.84323 & 0.19528 & 4.318\end{array}$




\section{Appendix 7. Information sheet for the participants of the experiment}

VICTORIA UNIVERSITY OF WELLINGTON

Te Whare Wananga o te Upoko o te Ika a Maui

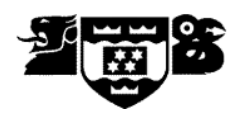

School of Linguistics and Applied Language Studies

PO Box 600, Wellington 6140

tel: 4635600

fax: 4635604

Researcher: Natalia Beliaeva: natalia.beliaeva@vuw.ac.nz

Supervisors: Laurie Bauer: laurie.bauer@vuw.ac.nz

Paul Warren: paul.warren@vuw.ac.nz

Anna Siyanova: anna.siyanova@vuw.ac.nz

\section{INFORMATION SHEET FOR PARTICIPANTS}

Thank you for participating in my study. My name is Natalia Beliaeva and I am a PhD student in Linguistics at Victoria University of Wellington in New Zealand. I am looking at how people understand new words that appear in English.

This project has been granted ethical approval by the Victoria University of Wellington Human Ethics Committee.

I am asking you to agree to take part in a linguistic experiment, during which you will complete two tasks. First, you will be shown a number of complex words on the computer screen and will be asked to guess what parts they consist of. Then, you will be shown a series of word-sized stimuli on the computer screen and will be asked to indicate for each stimulus whether it is a word of English or not by pressing one of two response buttons.

The experiment will take about 15 minutes. In acknowledgement of your time and effort you will receive a $\$ 10$ gift voucher.

The study is strictly confidential, i.e. your name will not be associated with any responses. The data will be used for my $\mathrm{PhD}$ thesis as well as for conference presentations based on the thesis research and for publication in academic journals. The thesis will be submitted for marking to Victoria University of Wellington and deposited in the University Library, after which it will become available electronically.

If you decide at any time during the experiment that you wish to withdraw from it, you may simply let me know and I will stop the experiment and will not use any of your responses. Once the experimental session is complete, I will not be able to identify your responses, and so will not at that stage be able to remove your responses.

At the end of the project, a summary report will be available, and if you wish to receive a copy you may request one by providing your email address, which will be used only for sending a report of the research and will not be associated with your responses. 


\section{Appendix 8. Consent form for the participants of the experiment}

VICTORIA UNIVERSITY OF WELLINGTON

Te Whare Wananga o te Upoko o te Ika a Maui

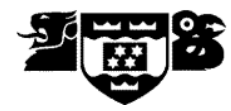

School of Linguistics and Applied Language Studies

PO Box 600, Wellington 6140

tel: 4635600

fax: 4635604

Researcher: Natalia Beliaeva: natalia.beliaeva@vuw.ac.nz

Supervisors: Laurie Bauer: laurie.bauer@vuw.ac.nz

Paul Warren: paul.warren@vuw.ac.nz

Anna Siyanova: anna.siyanova@vuw.ac.nz

\section{CONSENT TO PARTICIPATE IN RESEARCH}

\section{Please read the following carefully and sign below if you agree to the terms.}

I understand the procedure of this research as set out in the information sheet. I have been informed that this project received ethical approval from the Victoria University of Wellington Human Ethics Committee. My participation in this research is voluntary.

I understand that the data I provide in the experimental session will be used for a research conducted in the School of Linguistics and Applied Language Studies at Victoria University of Wellington. I also understand that this research is strictly confidential and that my name will not be associated with any responses, and that all personal information, e.g. age or first language, will be collected for statistical reasons only and will not be linked to the identities of individual participants. I also understand that if I wish to withdraw from the experiment during the experimental session, I can do so without giving reasons, and my responses will not be used for the research.

I understand that, on my request, a summary of results from the study will be sent to me at a later date.

\section{I have read and understood the consent form and I agree to its terms:}

Name (please print):

Signature:

Date:

Please tick here if you would like to receive a copy of summary report of the study:

Email address to which the report can be sent:

(the e-mail address will not be associated with your responses and will not be used for any purposes other than sending a report of the research). 


\section{Appendix 9. Experimental stimuli}

Task 1 (the stimuli on the grey background were used as warm-up fillers, and were not included in the analysis of the results):

\begin{tabular}{ll}
\hline Group 1 & Group 2 \\
\hline weisure & weisure \\
squintern & squintern \\
virtopsy & pickade \\
rumint & scigov \\
flotsametrics & guitarthritis \\
adorapresh & hydfrac \\
slacktivism & baggravation \\
chugger & renoviction \\
collabulary & femcho \\
scoratorium & blizzaster \\
hydrail & foco \\
clapathy & flabdomen \\
predictionary & dotcomrade \\
briet & negatude \\
textrovert & stoption \\
acatramp & globfrag \\
finlit & spagbol \\
\hline
\end{tabular}


Task 2:

\begin{tabular}{llll}
\hline \multicolumn{1}{l}{ Words } & & NonWords \\
\hline GrOUP A & GrOUP B & GrOUP A & Group B \\
\hline LEISURE & WORK & RINTAIN & SQUAILT \\
\hline WORK & LEISURE & SQUAILT & RINTAIN \\
HYDROGEN & RAILWAY & RAILDAWS & HYDREGOL \\
FOOD & COURT & COALX & FONK \\
PRECIOUS & ADORABLE & ADORAUZE & PRECSOUD \\
FRACTURING & HYDRAULIC & HYDRESOL & FRACSEDDING \\
FINANCIAL & LITERACY & LITRUVEY & FINERNIEL \\
SPAGHETTI & BOLOGNESE & BOLERLEAD & SPAGLINGO \\
INTELLIGENCE & RUMOUR & RUMACKS & INTRENIEWING \\
GOVERNMENT & SCIENCE & SCINEILL & GOVIMPTERN \\
ACADEMIC & TRAMPOLINE & TRAMPENOIT & ACALUCHER \\
GLOBALISATION & FRAGMENTATION & FRAGLIENSES & GLOBERTOTEING \\
DIET & BRIDE & BRILK & GIET \\
MACHO & FEMALE & FEMURCE & ANCHO \\
COLLABORATIVE & VOCABULARY & SCAURUBLARY & COLLABICATION \\
NEGATIVE & ATTITUDE & GARTITUDE & NEGACEDE \\
MUGGER & CHARITY & CHAFELET & STUGGER \\
DISASTER & BLIZZARD & BLIZANT & COLASTER \\
VIRTUAL & AUTOPSY & REITOPSY & VIRTOCKAN \\
PICKET & BLOCKADE & RACKADE & PICKNELL \\
MORATORIUM & SCORE & SCORT & DROATORIUM \\
EVICTION & RENOVATION & RENLUIRTION & CIVICTION \\
FLOTSAM & METRICS & MERTICS & FLOTASM \\
GUITAR & ARTHRITIS & RAHRITIS & GIURTAR \\
DICTIONARY & PREDICTION & REPDICTION & DOCTOILARY \\
COMRADE & DOTCOM & DOCTOM & COMHAED \\
SLACK & ACTIVISM & ARCOVISM & SLARK \\
BAG & AGGRAVATION & GARRAVATION & CHAG \\
APATHY & CLAP & CALP & APALTY \\
ABDOMEN & FLAB & FALB & ADOUMEN \\
TEXT & OPTROVERT & XEROVERT & TWEXT \\
STOP & OLPION & TOSP \\
\hline
\end{tabular}




\section{Appendix 10. Item variables used for the analysis of experimental data}

$\underline{\text { Task1 }}$

\begin{tabular}{|c|c|}
\hline Variable name & Variable description \\
\hline Group & Whether the prime was shown in Group 1 or Group 2 list \\
\hline Length & $\begin{array}{l}\text { The length of the prime (the number of letters/phonemes in the } \\
\text { blend or clipping compound used as prime in Task 1) }\end{array}$ \\
\hline w1length & The number of letters in $\mathrm{W} 1$ of the stimulus word \\
\hline w2length & The number of letters in W2 of the stimulus word \\
\hline Sp1Length & The number of letters in the first splinter of the prime \\
\hline Sp2Length & The number of letters in the second splinter of the prime \\
\hline Freq1 & $\begin{array}{l}\text { The frequency of W1 of the prime in COCA (log transformed for the } \\
\text { analyses) }\end{array}$ \\
\hline Freq2 & $\begin{array}{l}\text { The frequency of W2 of the prime in COCA (log transformed for the } \\
\text { analyses) }\end{array}$ \\
\hline PrimeGoogleFreq & Google frequency of the prime (log transformed for the analyses) \\
\hline PrimeType & Structural type of the prime: $\mathrm{AC}, \mathrm{AD}$, or $\mathrm{WW}$ \\
\hline FreqSp1 & $\begin{array}{l}\text { The cumulative frequency of all words in COCA that begin with the } \\
\text { first splinter of the prime }\end{array}$ \\
\hline FreqSp2 & $\begin{array}{l}\text { The cumulative frequency of all words in COCA that begin with the } \\
\text { second splinter of the prime (if the prime is an AC form), or end with } \\
\text { that splinter (if the prime is an AD or WW blend) }\end{array}$ \\
\hline Similarity1 & $\begin{array}{l}\text { the Weber and van Orden }(1987: 196) \text { spelling distance between W1 } \\
\text { and the prime }\end{array}$ \\
\hline Similarity2 & $\begin{array}{l}\text { the Weber and van Orden }(1987: 196) \text { spelling distance between W2 } \\
\text { and the prime }\end{array}$ \\
\hline RelFreq1 & $\begin{array}{l}\text { The relative frequency of the first splinter of the prime, calculated as } \\
\text { FreqSp1 / Freq1 }\end{array}$ \\
\hline RelFreq2 & $\begin{array}{l}\text { The relative frequency of the first splinter of the prime, calculated as } \\
\text { FreqSp } 2 \text { / Freq } 2\end{array}$ \\
\hline SPD1 & $\begin{array}{l}\text { The switch point distance of the first splinter, i.e. the distance } \\
\text { between the actual switch point and the uniqueness point of W1, } \\
\text { calculated using the method developed in Gries (2006) }\end{array}$ \\
\hline SPD2 & $\begin{array}{l}\text { The switch point distance of the second splinter, i.e. the distance } \\
\text { between the actual switch point and the uniqueness point of W2, } \\
\text { calculated using the method developed in Gries (2006) }\end{array}$ \\
\hline
\end{tabular}




\begin{tabular}{ll}
\hline Variable name & Variable description \\
\hline PrimeShown & Whether the prime for this target was shown to this group of \\
participants or not & Whether the target is a word or not \\
Tword & The length of the target in letters \\
Tlength & Log-transformed COCA frequency of target \\
LogTfreq & Which source word of the prime is target (1 if target is the \\
PrSW & The length of the prime in letters \\
Plength & Log-transformed Google frequency of prime \\
TogPfreq & The structural type of prime (AC, AD, WW) \\
PrimeType & The length of the splinter in letters (how many letters from \\
target are contained in prime) & The Weber and van Orden spelling distance between target \\
and prime & The switch point distance relevant for the target, i.e. the \\
distance between the actual switch point in the & corresponding prime, and the uniqueness point of target, \\
Pspd & calculated using the method developed in Gries (2006) \\
& The relative splinter frequency of the corresponding splinter \\
of the prime, calculated by dividing the cumulative frequency \\
of words beginning/ending with the splinter, using the \\
method suggested in Cook and Stevenson (2007) \\
The Weber and van Orden spelling distance between target \\
and the relevant source word of prime (measured for \\
nonword targets only) \\
The proportion of the corresponding target word retained in \\
the nonword target, calculated as the length of the letter \\
string from target word preserved in the nonword divided by \\
the length of the target word (measured for nonword targets \\
only)
\end{tabular}




\section{Appendix 11. Regression models predicting the response to the experimental stimuli}

Task 1.

\section{(A11.1) The model predicting SW1 naming}

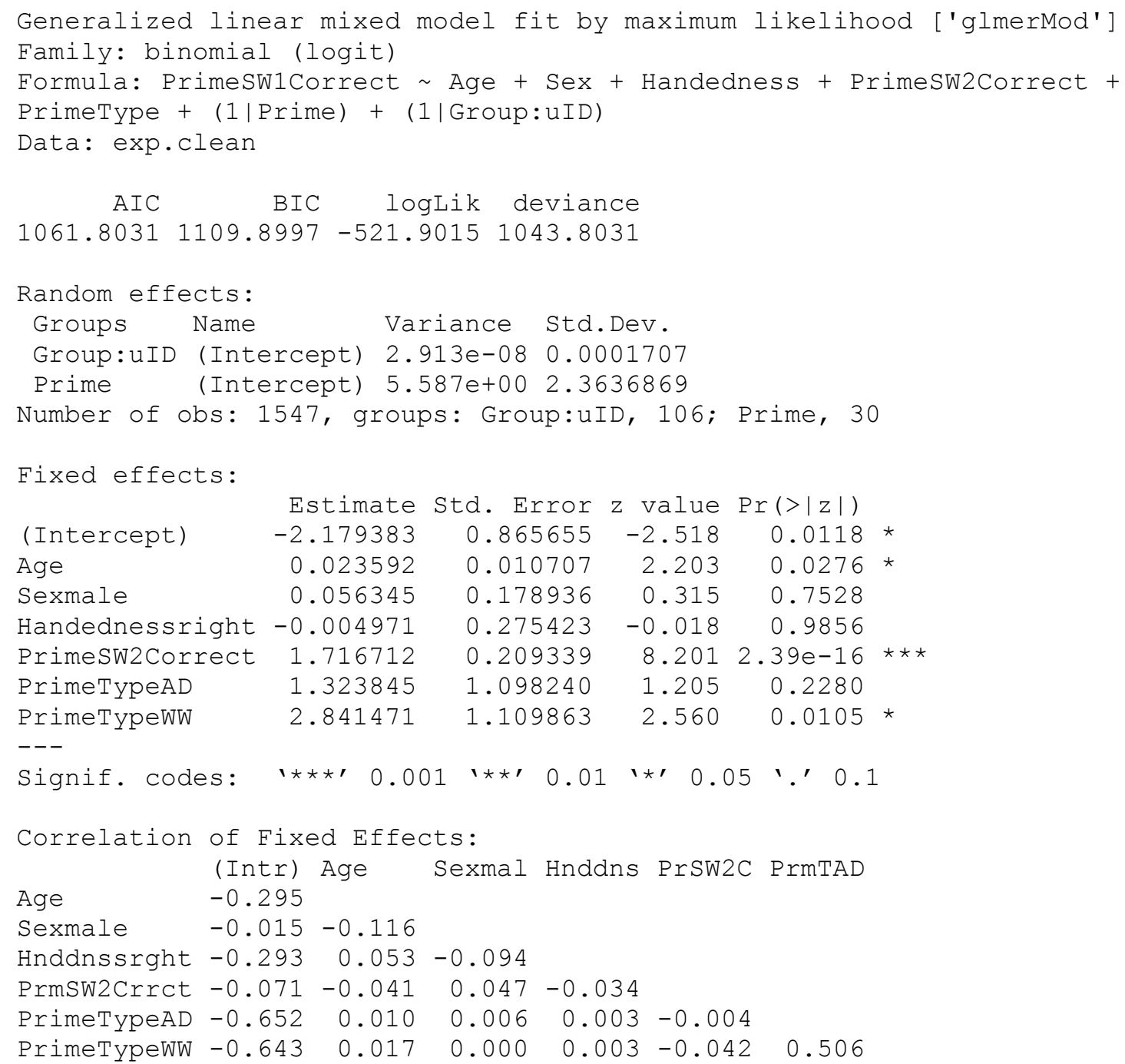

\section{(A11.2) The model predicting SW2 naming}

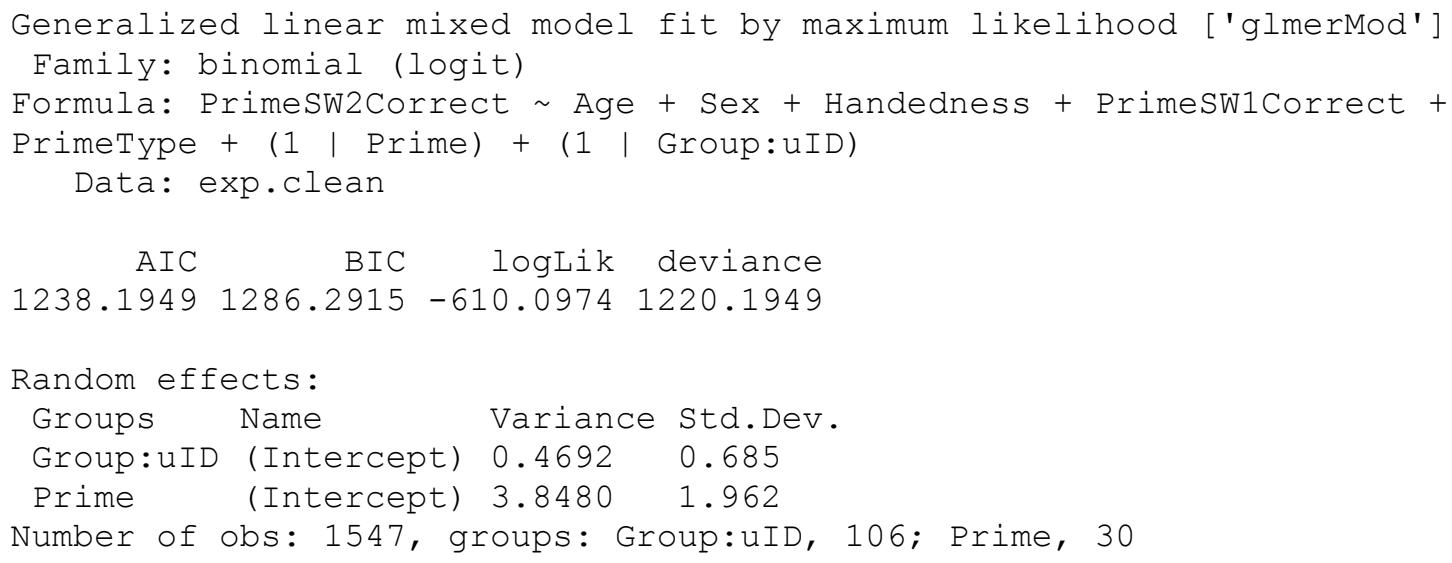


Fixed effects:

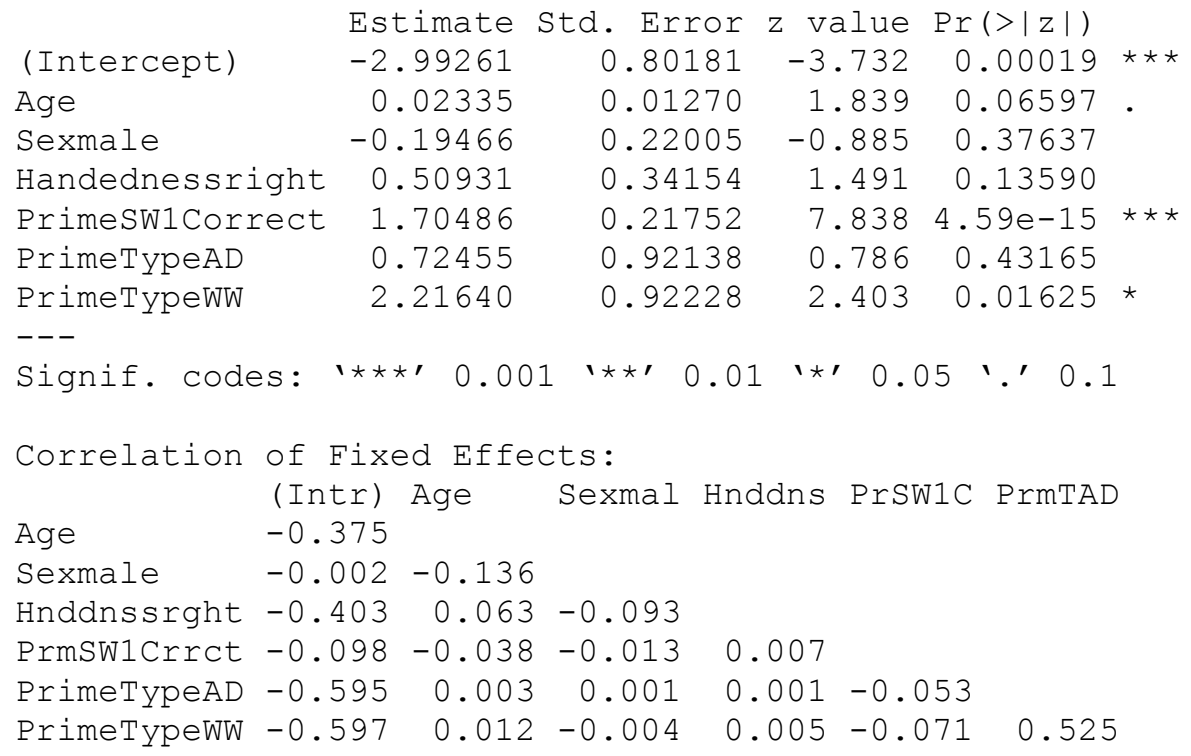

\section{(A11.3) The model predicting SW2 naming in Group 1}

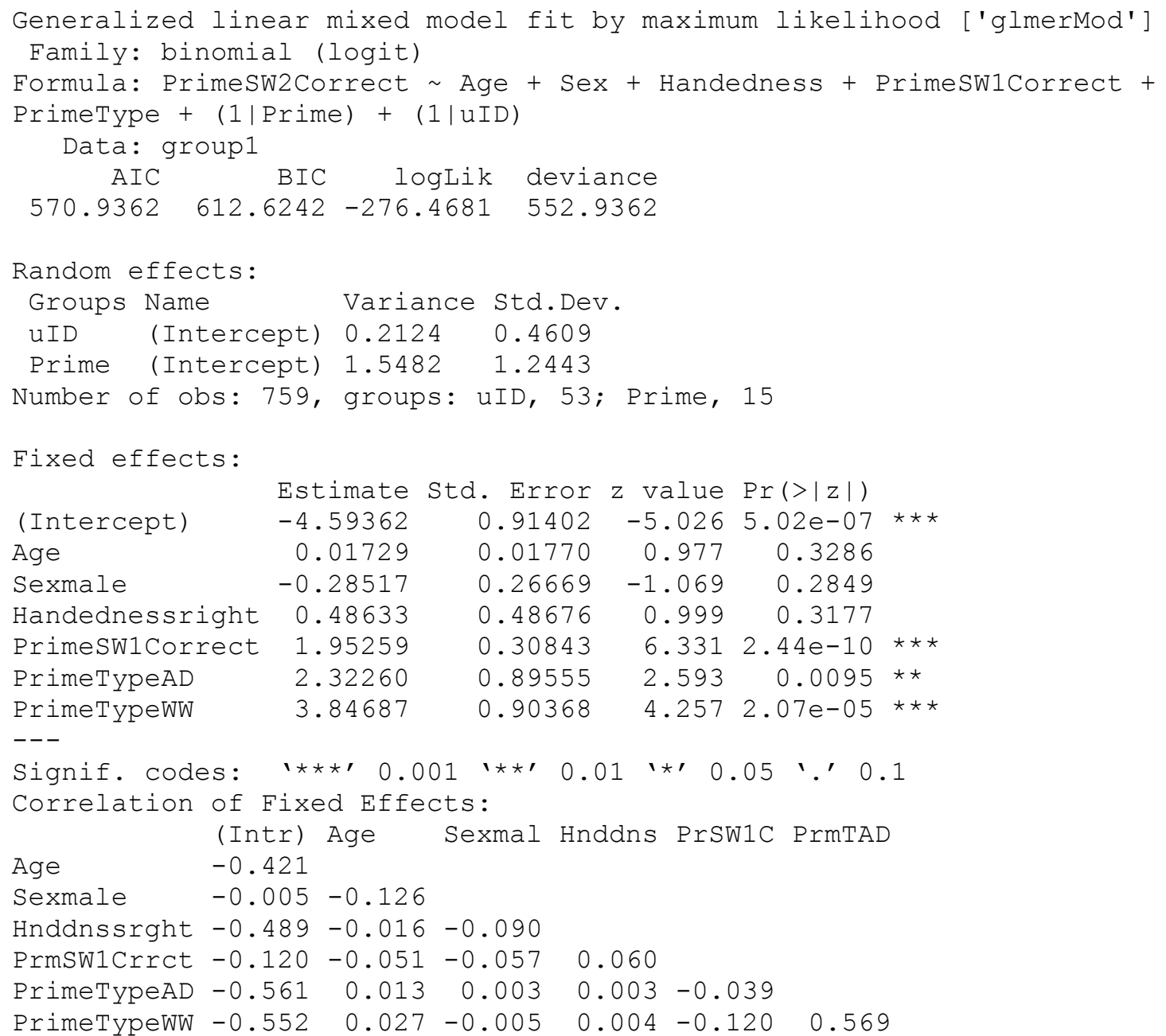




\section{(A11.4) The model estimating the effect of relative splinter frequency on SW1 naming}

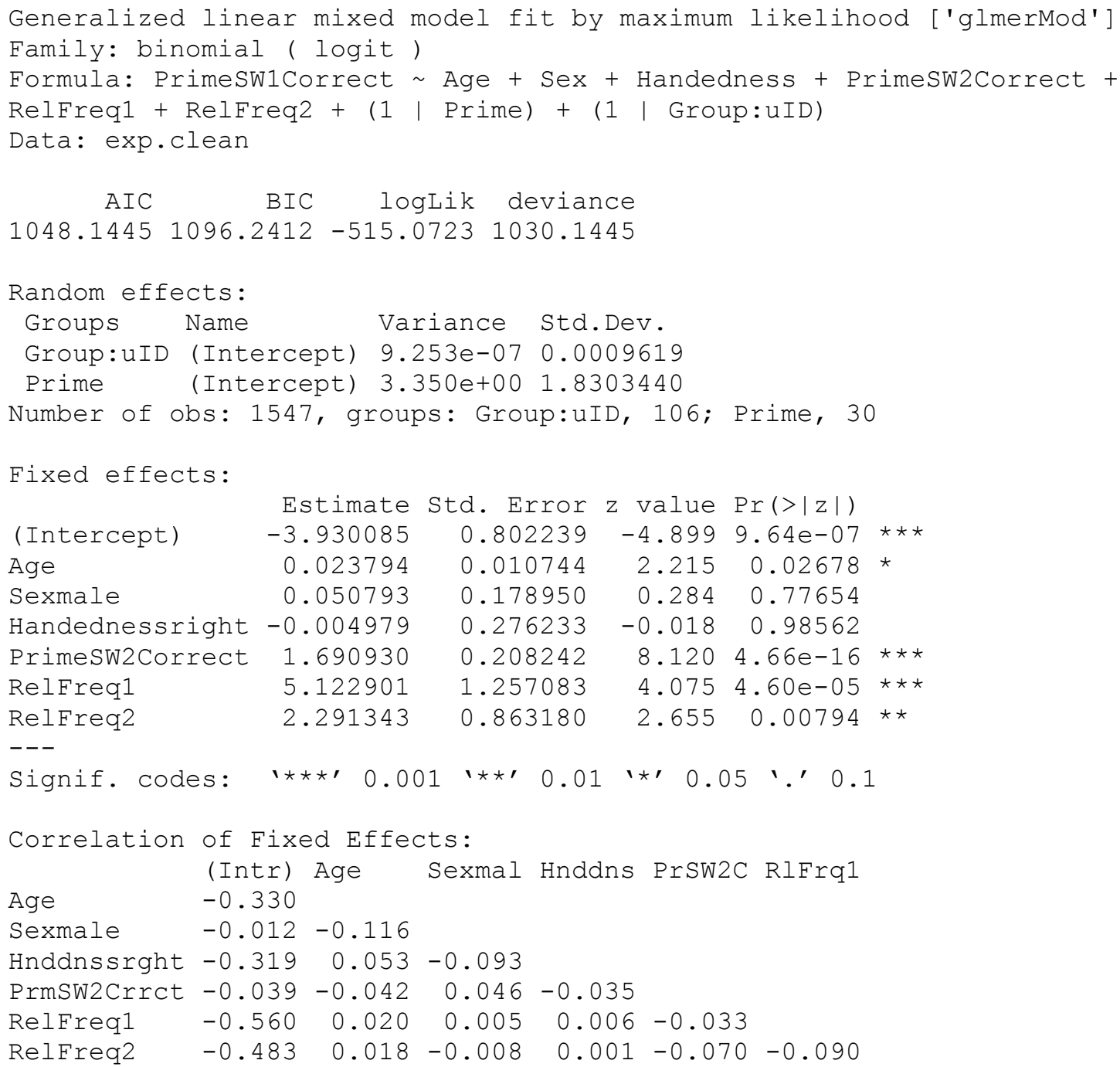

\section{(A11.5) The model estimating the effect of relative splinter frequency on SW2 naming}

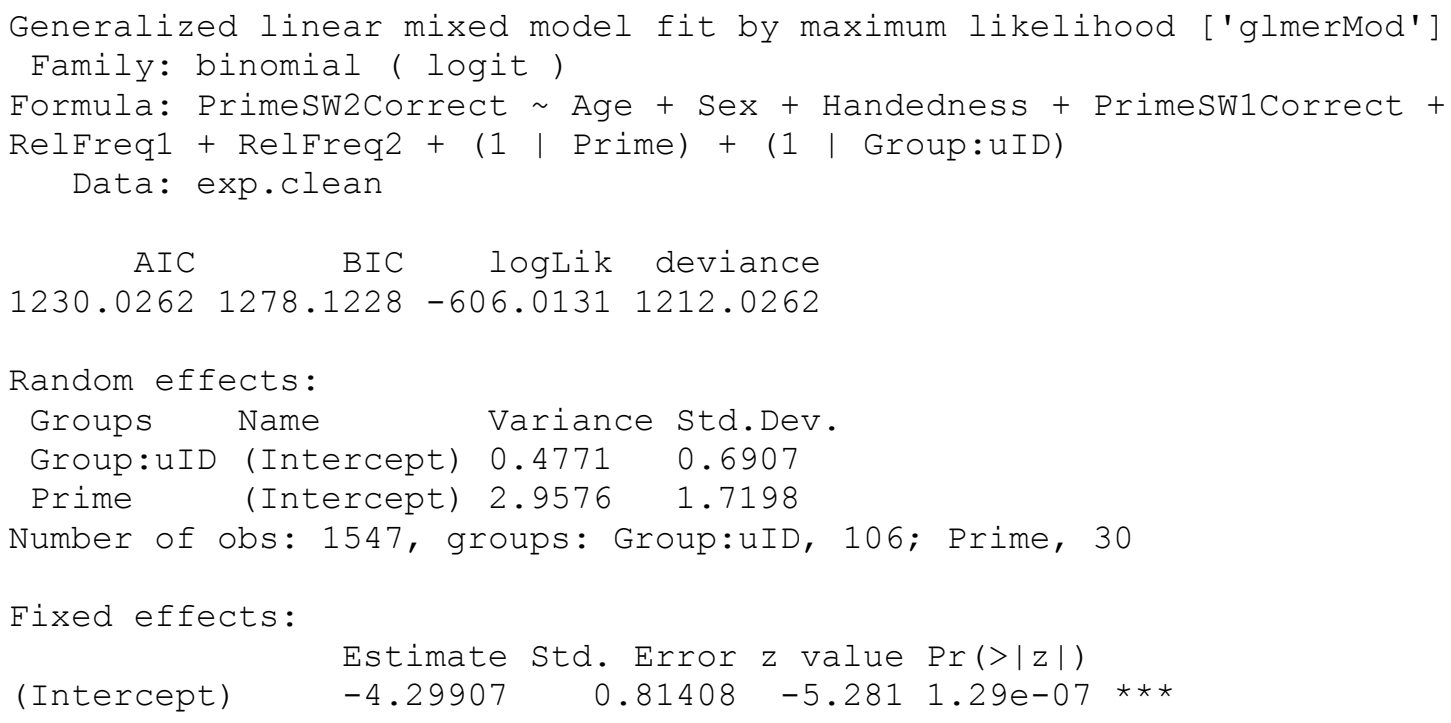




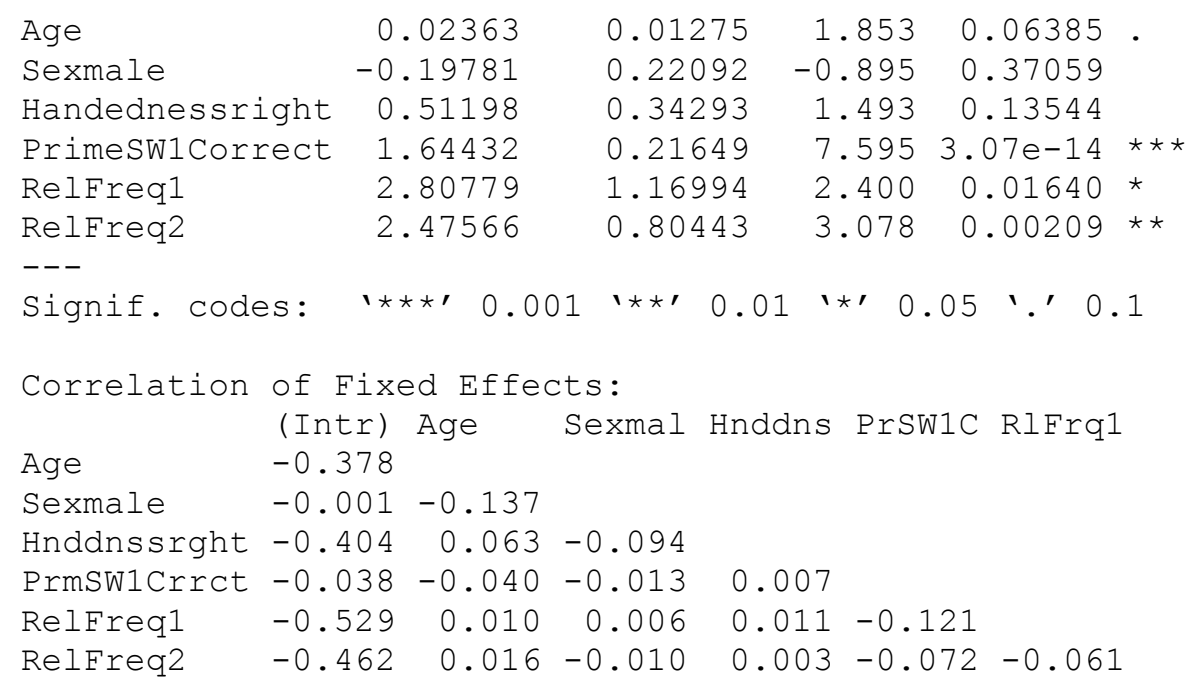

\section{(A11.6) The model predicting SW1 naming, which includes a set of item and participant variables}

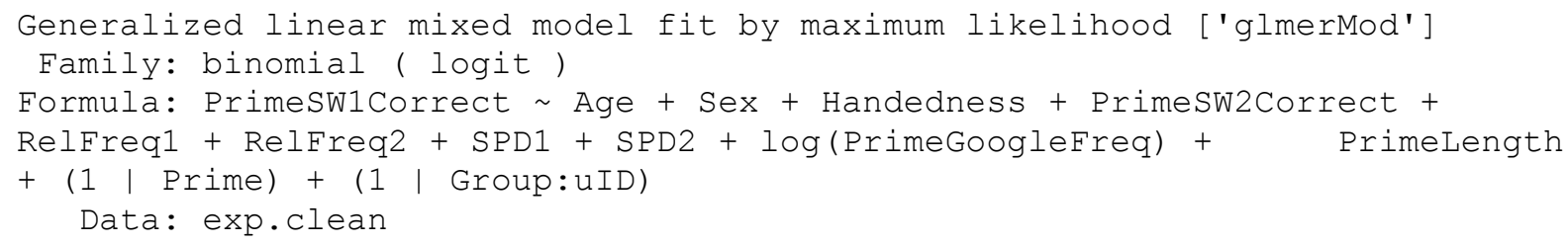




\begin{tabular}{|c|c|c|c|c|c|c|c|c|c|}
\hline RelFreq2 & -0.310 & 0.008 & 0.002 & -0.002 & -0.072 & 0.463 & & & \\
\hline SPD1 & 0.054 & -0.006 & -0.008 & 0.001 & 0.012 & -0.775 & -0.298 & & \\
\hline SPD2 & 0.614 & 0.001 & 0.001 & 0.001 & 0.057 & 0.027 & -0.625 & -0.270 & \\
\hline $\lg (\operatorname{PrmGglF})$ & -0.610 & 0.001 & -0.003 & 0.000 & -0.049 & -0.199 & -0.124 & 0.333 & -0.287 \\
\hline $\begin{array}{l}\text { PrimeLength } \\
0.190\end{array}$ & -0.635 & 0.005 & -0.007 & 0.001 & -0.033 & -0.401 & -0.180 & 0.159 & -0.310 \\
\hline
\end{tabular}

\section{(A11.7) The model predicting SW1 naming, which includes a residualised term for the effect of SPD not accounted for relative splinter frequency}

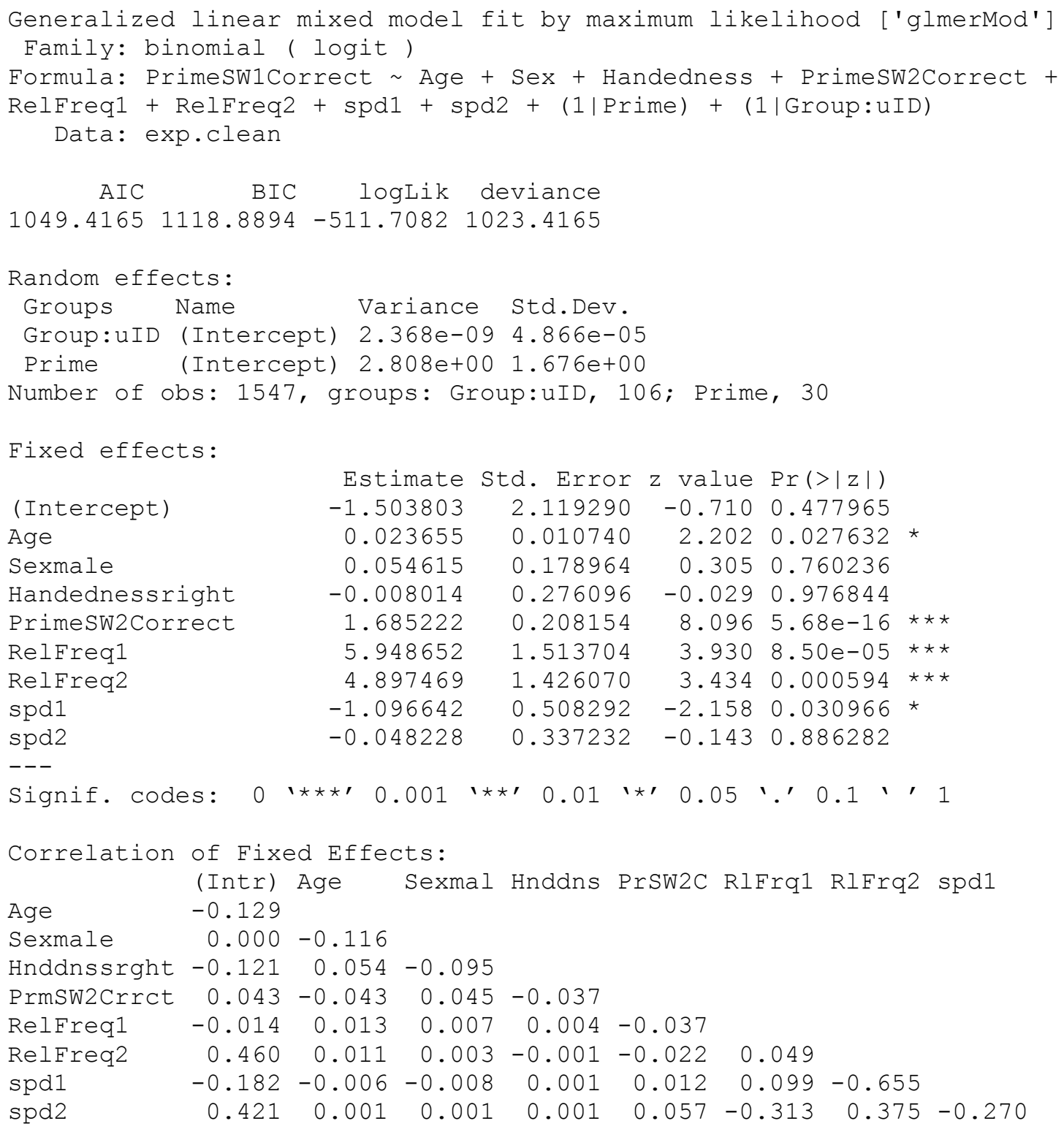




\section{(A11.8) The model predicting SW1 naming, which includes a residualised term for the effect of SPD not accounted for relative splinter frequency, and also includes the interaction of prime type and relative splinter frequency}

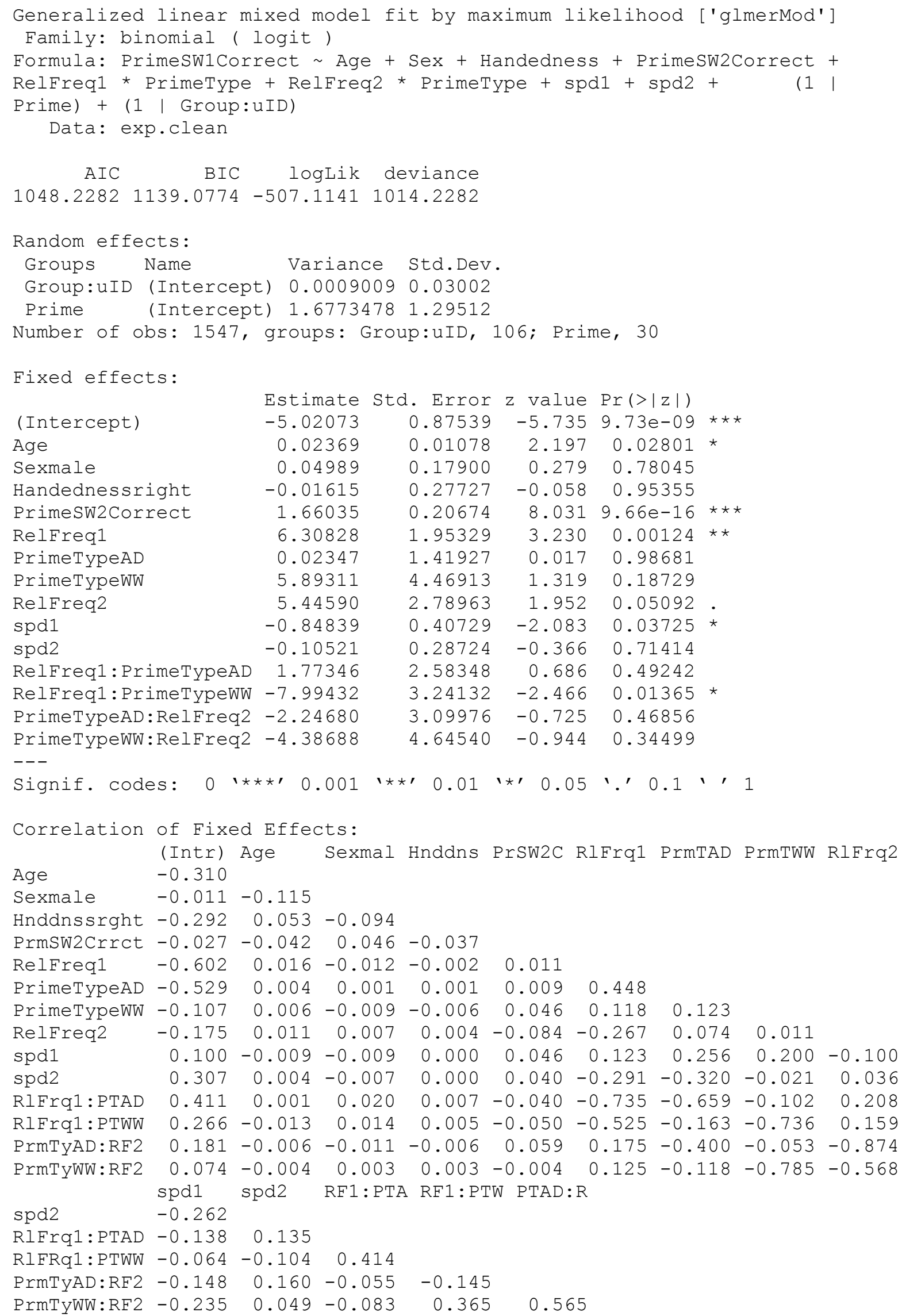




\section{(A11.9) The model predicting SW2 naming}

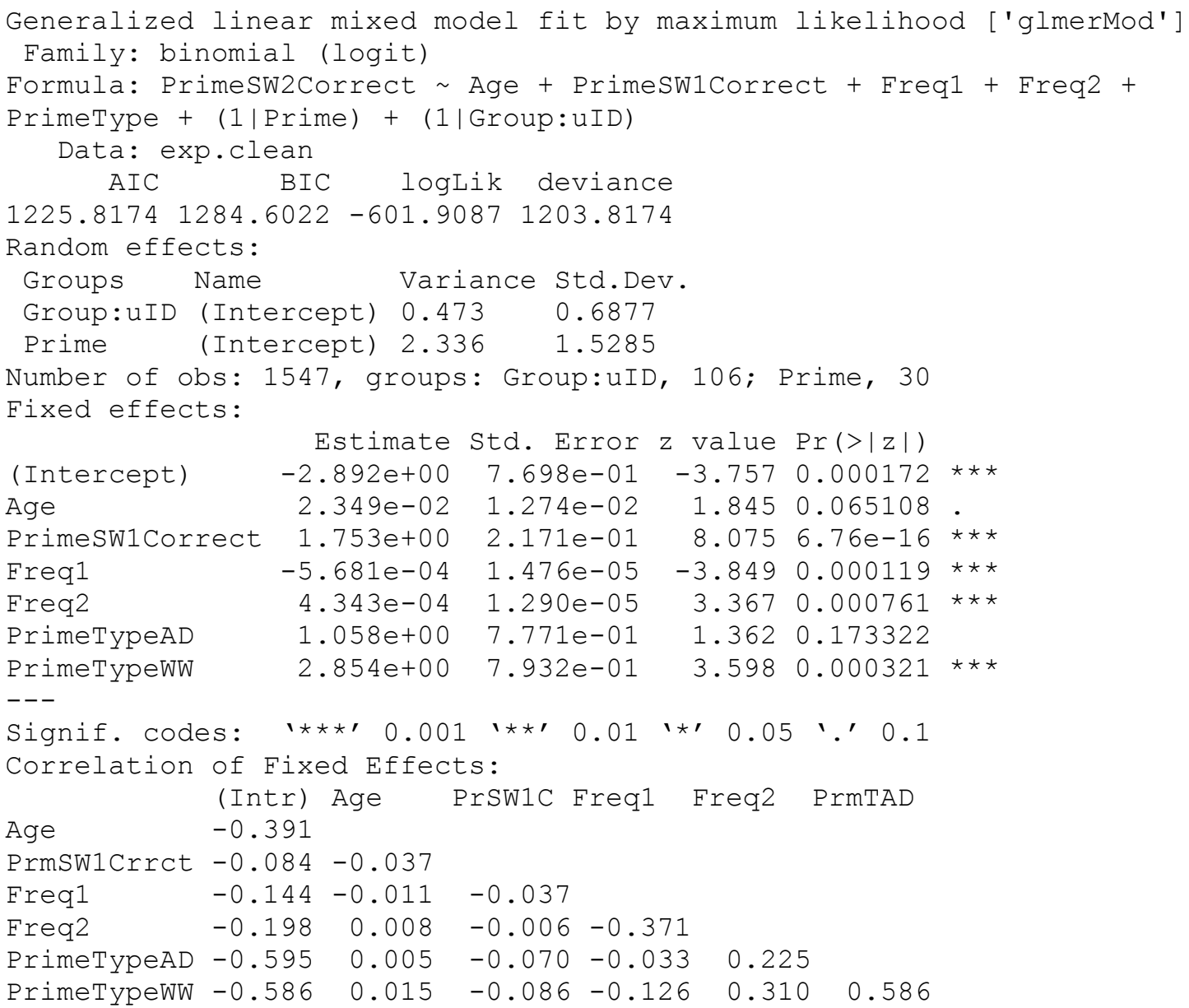

\section{(A11.10) The model predicting RT in Task 1}

Linear mixed model fit by REML

Formula: Stimulus1.RT PrimeLength + PrimeSW1Correct * PrimeSW2Correct +

(1|Prime) + (1+PrimeLength|uID)

AIC BIC logLik deviance REMLdev

$2903729096-14508 \quad 29092 \quad 29015$

Random effects:

$\begin{array}{llrrl}\text { Groups } & \text { Name } & \text { Variance } & \text { Std.Dev. Corr } \\ \text { uID } & \text { (Intercept) } & 7141993 & 2672.5 & \\ & \text { PrimeLength } & 33598 & 183.3 & -0.884 \\ \text { Prime } & \text { (Intercept) } & 1879417 & 1370.9 & \\ \text { Residual } & & 7192664 & 2681.9 & \end{array}$

Number of obs: 1547, groups: uID, 106; Prime, 30

Fixed effects:

(Intercept)

PrimeLength

PrimeSW1Correct

Primesw2Correct

PrimeSW1Correct: PrimeSW2Correct

Correlation of Fixed Effects:

(Intr) PrmLng PrSW1C PrSW2C

PrimeLength -0.936

PrmSW1Crrct $0.005-0.129$

$\begin{array}{llll}\text { PrmSW2Crrct } \quad 0.002 & -0.101 & 0.350\end{array}$

PSW1C:PSW2C $0.016 \quad 0.047 \quad-0.610-0.773$

$\begin{array}{rrr}\text { Estimate Std. Error t value } \\ 5819.7 & 1003.6 & 5.799 \\ 304.2 & 110.6 & 2.751 \\ -3311.7 & 278.8 & -11.877 \\ -2657.0 & 326.1 & -8.147 \\ 1206.8 & 389.2 & 3.101\end{array}$


(A11.11) The model predicting RT to words in Task 2, with simple effects only
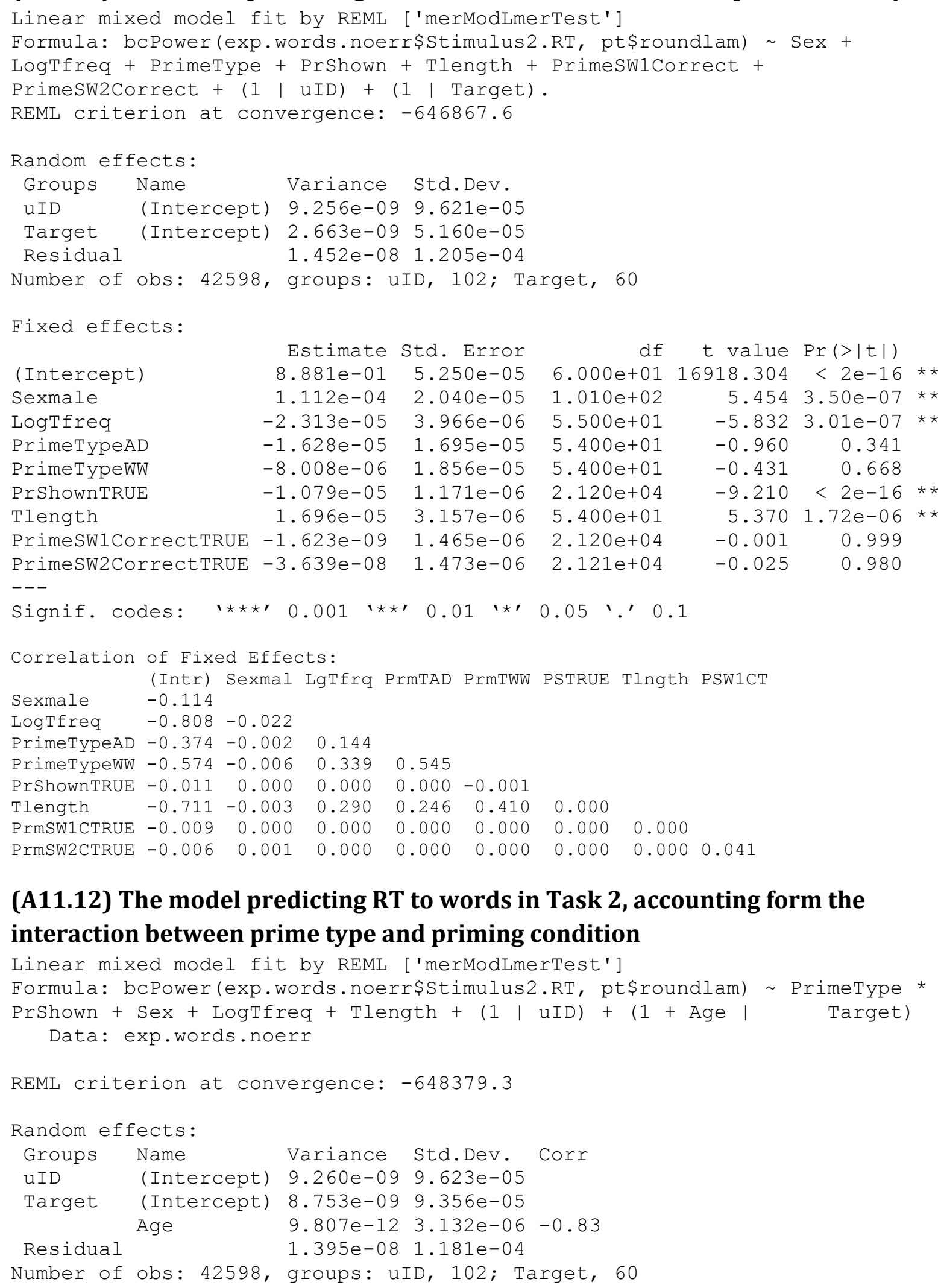


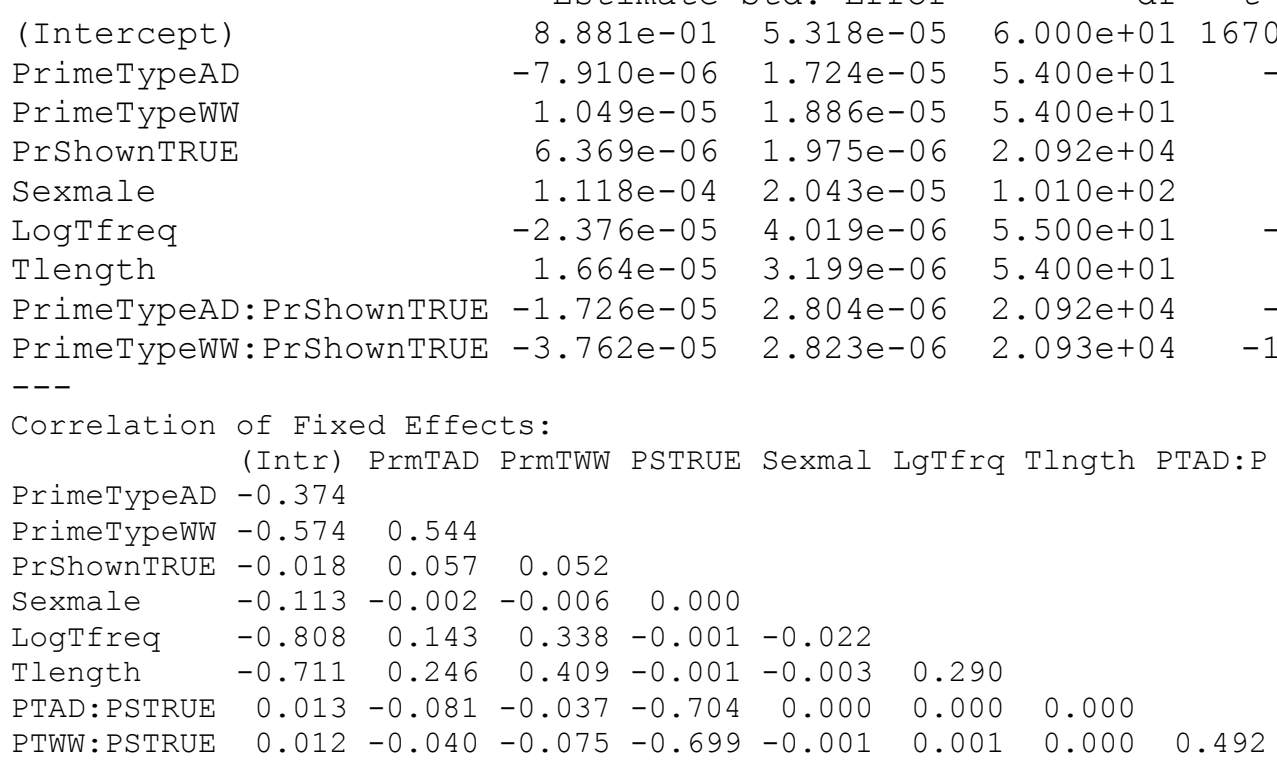

\section{(A11.13) The final model predicting RT to words in Task 2}

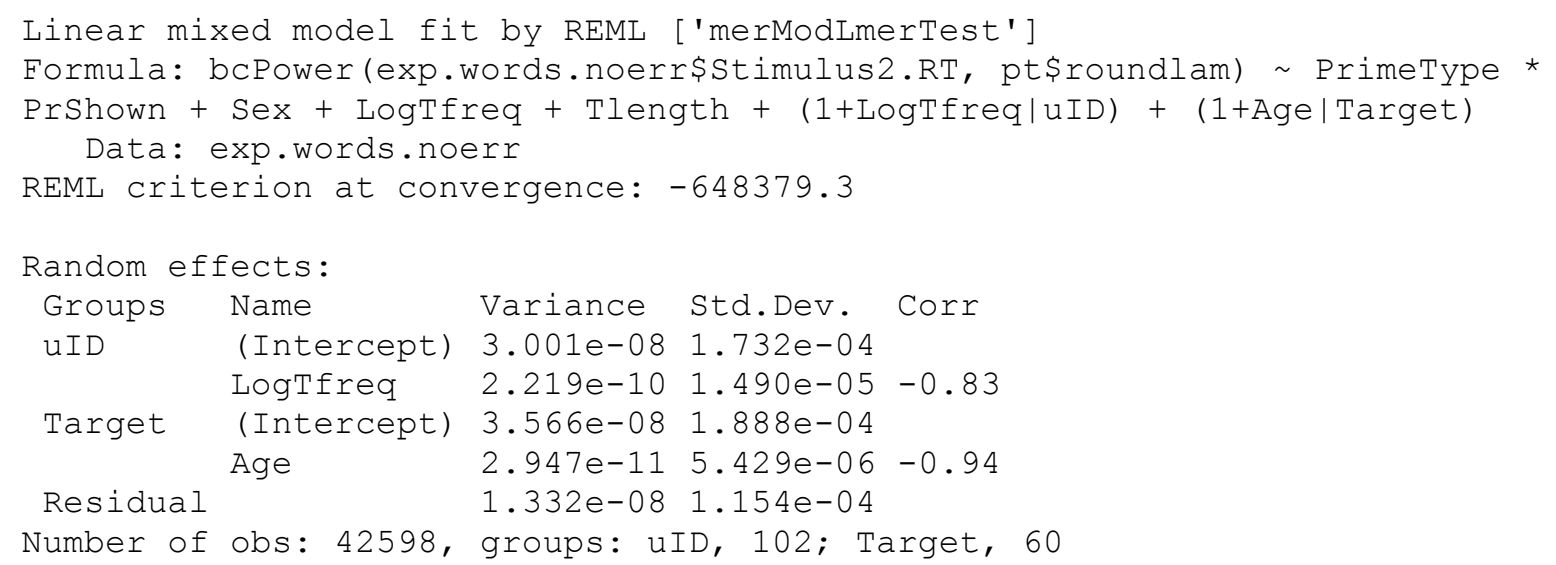

Fixed effects:

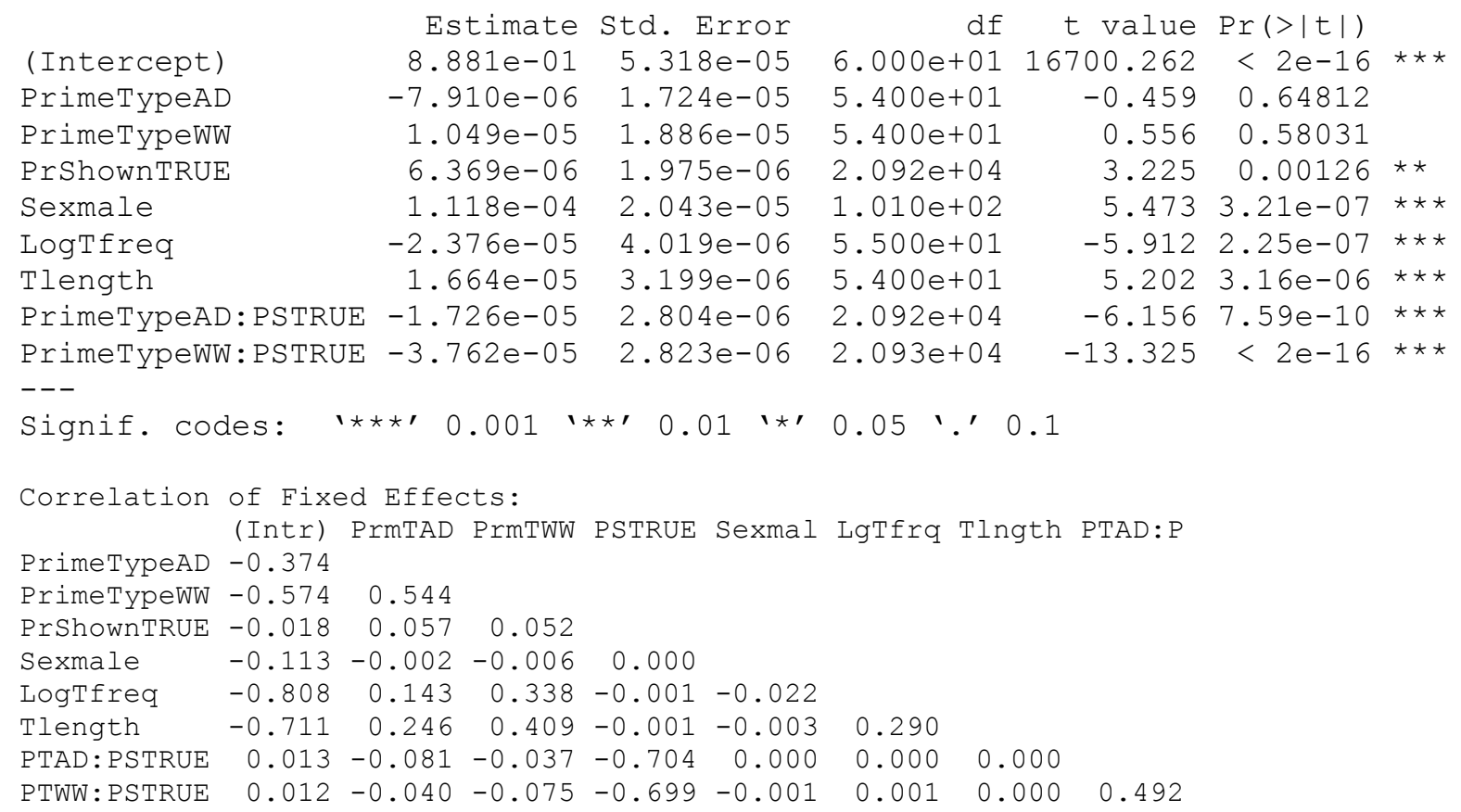




\section{(A11.14) The model predicting RT to nonwords in Task 2 , with simple effects only}

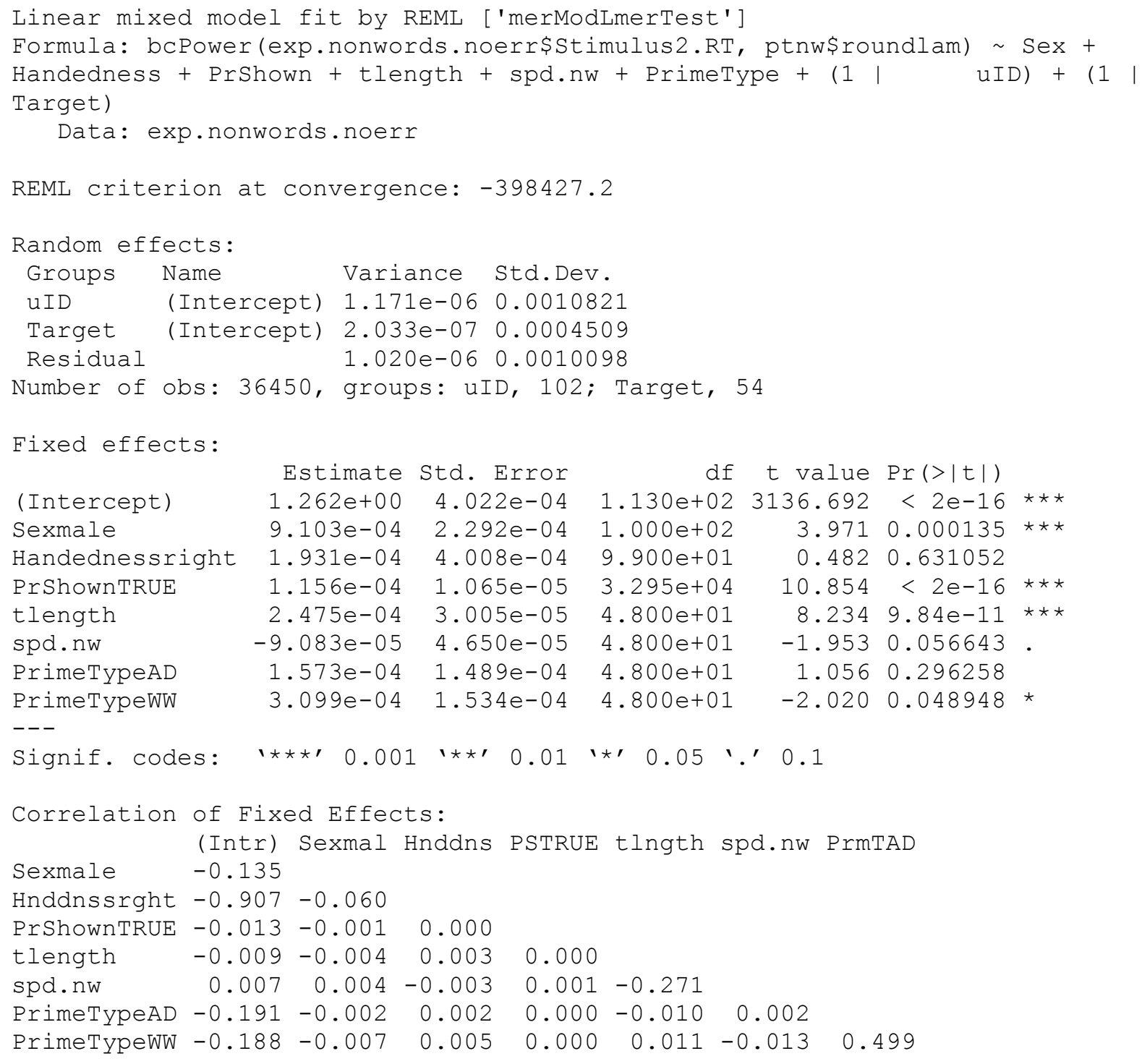


Fixed effects:

\begin{tabular}{|c|c|c|c|c|c|c|}
\hline & Estimate & Std. Error & $d f$ & t value & $\operatorname{Pr}(>|t|)$ & \\
\hline (Intercept) & $1.262 e+00$ & $1.706 e-04$ & $1.460 e+02$ & 7397.691 & $<2 e-16$ & 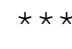 \\
\hline Sexmale & $9.596 e-04$ & $2.245 e-04$ & $1.000 e+02$ & 4.275 & $4.39 e-05$ & $\star \star \star *$ \\
\hline PrShownTRUE & $1.785 e-04$ & $1.843 e-05$ & $3.281 e+04$ & 9.689 & $<2 e-16$ & $\star \star \star$ \\
\hline PrimeTypeAD & $2.153 e-04$ & $1.502 e-04$ & $4.900 e+01$ & 1.433 & 0.158087 & \\
\hline PrimeTypeWW & $2.668 e-04$ & $1.567 e-04$ & $5.100 e+01$ & -1.702 & 0.094718 & . \\
\hline tlength & $2.485 e-04$ & $3.022 e-05$ & $4.800 e+01$ & 8.223 & $9.56 e-11$ & $\star \star \star$ \\
\hline spd.nw & $-9.084 e-05$ & $4.676 e-05$ & $4.800 e+01$ & -1.943 & 0.037906 & . \\
\hline PSTRUE: PrimeTypeAD & $1.169 e-05$ & $2.605 e-05$ & $3.283 e+04$ & -4.489 & $7.20 e-06$ & $\star \star \star$ \\
\hline $\begin{array}{l}\text { PSTRUE : PrimeTypeWh } \\
---\end{array}$ & $9.573 e-05$ & $2.615 e-05$ & $3.286 e+04$ & -3.660 & 0.000252 & $\star \star \star ⿻$ \\
\hline
\end{tabular}

Correlation of Fixed Effects:

\begin{tabular}{|c|c|c|c|c|c|c|c|c|}
\hline & (Intr) & Sexmal & PrSTRUE & PrmTAD & PrmTWW & tlngth & spd.nw & PSTRUE : PTA \\
\hline Sexmale & -0.439 & & & & & & & \\
\hline rShownTRUE & -0.053 & -0.002 & & & & & & \\
\hline rimeTypeAD & -0.460 & -0.002 & 0.063 & & & & & \\
\hline rimeTypeWW & -0.458 & -0.008 & 0.056 & 0.501 & & & & \\
\hline Iength & -0.029 & -0.003 & -0.001 & -0.005 & 0.022 & & & \\
\hline od.nw & -0.001 & 0.003 & 0.001 & 0.006 & 0.000 & -0.265 & & \\
\hline STRUE : PTAD & 0.039 & 0.001 & -0.720 & -0.087 & -0.040 & 0.002 & -0.001 & \\
\hline STRUE : PTWW & 0.037 & -0.002 & -0.667 & -0.042 & -0.084 & 0.001 & -0.001 & 0.484 \\
\hline
\end{tabular}

\section{(A11.16) The model predicting error rate for words in Task 2}

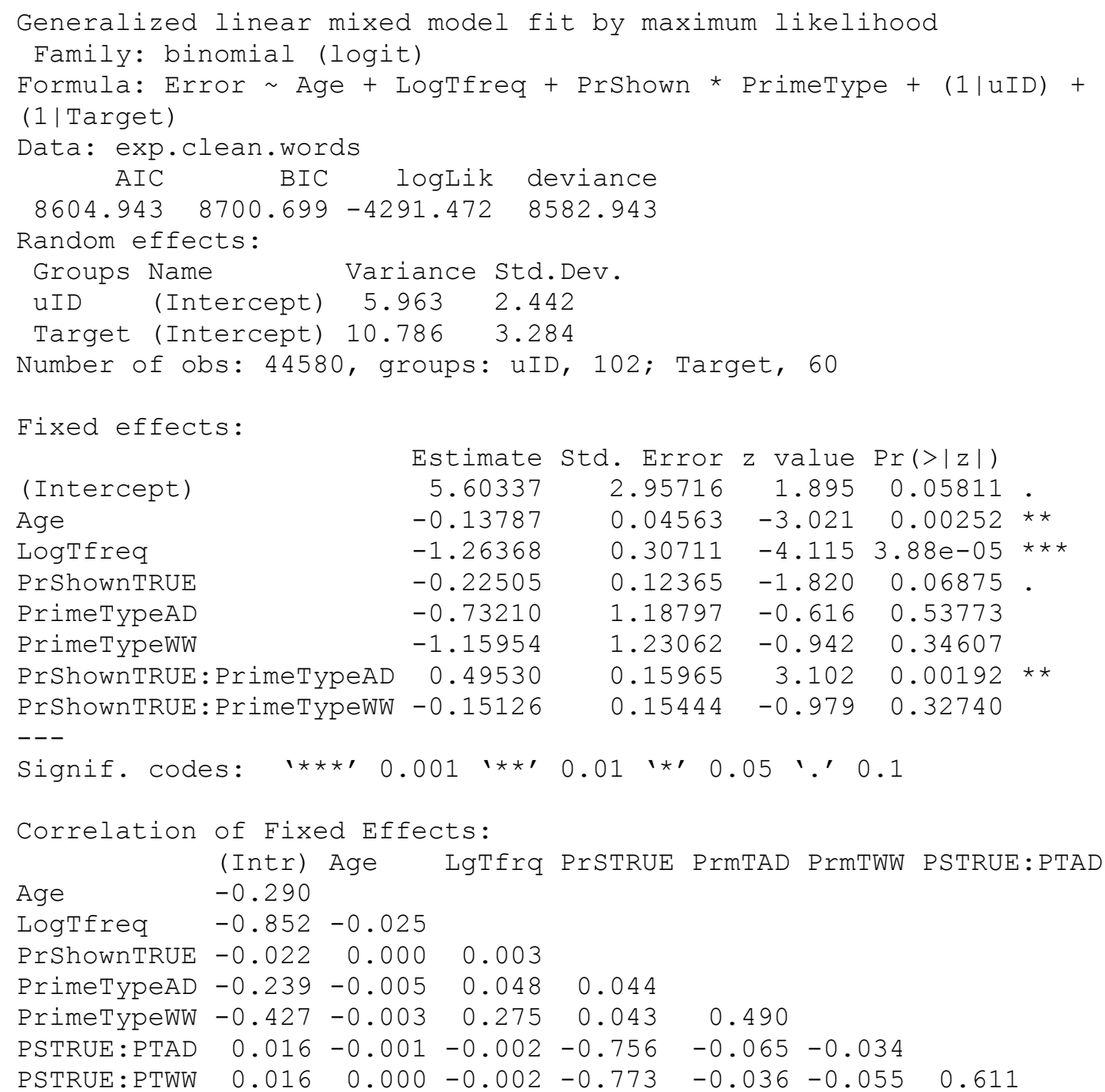




\section{(A11.17) The model predicting error rate for nonwords in Task 2}

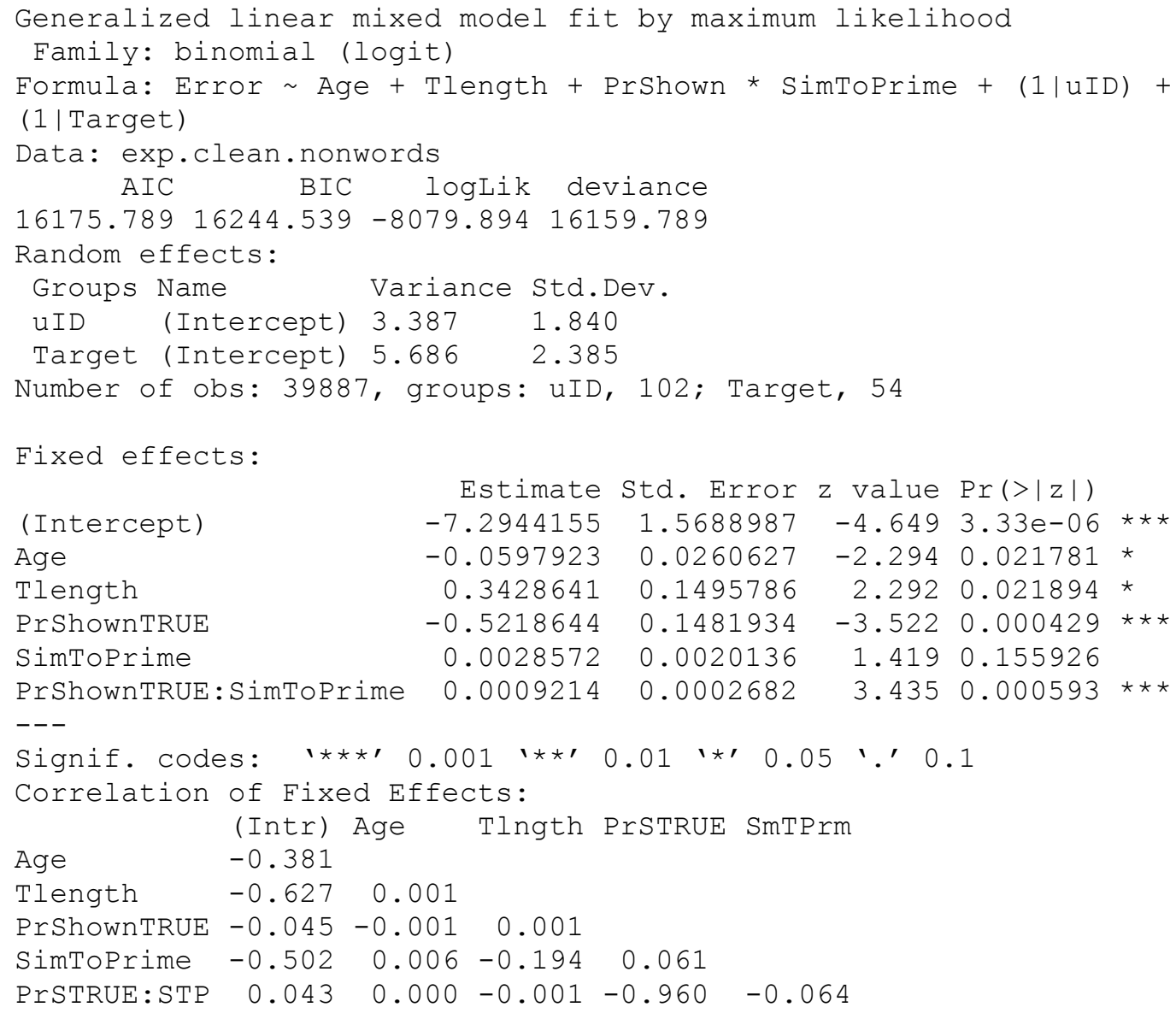

